\title{
MOLECULAR CHARACTERIZATION OF \\ A CONGENITAL OVERGROWTH SYNDROME INDUCED \\ BY ASSISTED REPRODUCTION
}

A Dissertation
presented to
the Faculty of the Graduate School
at the University of Missouri-Columbia

In Partial Fulfillment

of the Requirements for the Degree

Doctor of Philosophy

\begin{abstract}
By
ZHIYUAN CHEN

Dr. Rocío Melissa Rivera, Dissertation Supervisor

MAY 2017
\end{abstract}


The undersigned, appointed by the dean of the Graduate School, have examined the dissertation entitled

\section{MOLECULAR CHARACTERIZATION OF A CONGENITAL OVERGROWTH SYNDROME INDUCED BY ASSISTED REPRODUCTION}

presented by Zhiyuan Chen

a candidate for the degree of Doctor of Philosophy

and hereby certify that, in their opinion, it is worthy of acceptance.

Dr. Rocío M. Rivera

Dr. Marisa Bartolomei

Dr. Christine Elsik

Dr. Tieming Ji

Dr. James Amos-Landgraf 


\section{ACKNOWLEDGEMENTS}

There are a lot of people I would like to thank. I would not have been able to obtain my Ph.D degree without their generous support. First, I want to thank Dr. Rocío Melissa Rivera, who has been an outstanding mentor to me. Her passion for my research and my career development are invaluable for my $\mathrm{Ph}$. D training. Next, I would like to thank my committee members Drs. Marisa Bartolomei, Christine Elsik, Tieming Ji, and James Amos-Landgraf for the guidance on my research projects and the encouragement for me to further pursue an academic career. Especially, I want to acknowledge Dr. Elsik and Dr. Ji for their assistance in bioinformatics and statistical analyses.

I want to thank Dr. Elsik's laboratory members Dr. Darren Hagen, Dr. Christopher Childers, Dr. Shu Tao, Colin Diesh, and Deepak Unni for their support in the computational analyses. They have spent tremendous time teaching me how to run Linux commands and how to debug Perl scripts. I also would like to acknowledge Dr. Ji's laboratory member Juanbin Wang for her assistance in statistical analyses using R.

I would like to thank my laboratory mates Colin Morris and Laura Moon for their invaluable help with the validation of allele-specific gene expression determined by RNAseq. Especially I want to acknowledge Kira Marshall for her critical reading of the PNAS manuscript and numerous abstracts. She has been very helpful in correcting grammar errors and fixing inappropriate jargons. Lastly, I want to thank current laboratory members Yahan Li, Logan Womack, and Olivia Styron for making the laboratory such a positive environment. 


\section{Table of Contents}

Acknowledgements ........................................................................................................................ ii

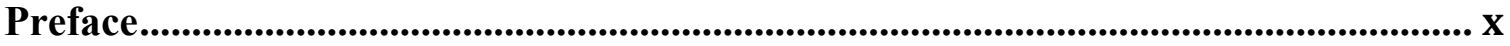

Nomenclature

Chapter 1 Literature review ....................................................................................................1

1.1 General overview of genomic imprinting......................................................................1

1.2 Epigenetic properties of imprinted genes.......................................................................3

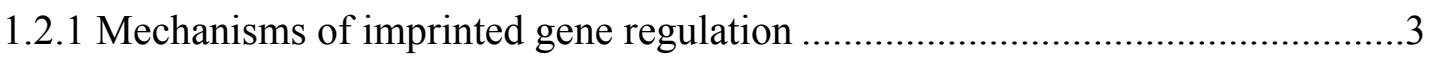

1.2.2 Dynamic regulation of genomic imprints .........................................................6

1.3 Functional roles of imprinted genes in mammalian development......................19

1.3.1 Imprinted gene network and pre- and post-natal development .........................19

1.3.2 Roles of imprinted genes in placenta...............................................................22

1.3.3 Roles of imprinted genes in behavior ...........................................................22

1.4 Human loss-of-imprinting disorders ..........................................................................23

1.4.1 Prader-Willi syndrome and Angelman syndrome ………................................23

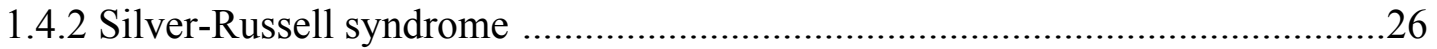

1.4.3 Beckwith-Wiedemann syndrome ……………………..............................26

1.4.4 Mouse models for Beckwith-Wiedemann syndrome ......................................28

1.5 Assisted reproductive technologies/therapies and genomic imprinting .............31

1.5.1 Assisted reproductive technologies can induce loss-of-imprinting in animals .31

1.5.2 Assisted reproductive technologies and Beckwith-Wiedemann syndrome .......33

1.6 Large offspring syndrome ........................................................................................34

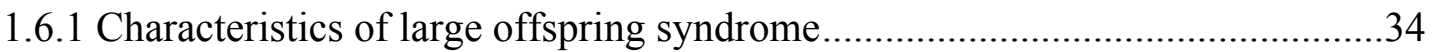

1.6.2 Molecular alterations of large offspring syndrome …….....................................36

1.7 Rationale .............................................................................................................................37

Chapter 2 Characterization of global loss of imprinting in fetal overgrowth syndrome induced by assisted reproduction .......................................................................41

2.1 Abstract ......................................................................................................................................41 
Figure 1. Loss-of-imprinting in large offspring syndrome (LOS)

Figure 2. Verification of the allelic expression of PEG3, NNAT, SNRPN, and

$P L A G L 1$ in control and $L O S$ fetuses .50

Figure 3. Allele-specific DNA methylation of the PLAGL1, SNRPN, and $N N A T$

DMRs in control and LOS fetuses

2.4 Discussion

Table 1. Shared descriptors of differentially expressed imprinted genes in LOS fetuses

2.5 Materials and methods

2.6 Acknowledgements.

2.7 Supplemental information for Chapter 2

Figure S1. Day 105 fetuses used in this study

Figure S2. Allele-specific expression analyses of known imprinted genes using RNAseq in brain of the fetuses.

Figure S3. Allele-specific expression analyses of known imprinted genes using RNAseq in liver of the fetuses

Figure S4. Allele-specific expression analyses of known imprinted genes using RNAseq in skeletal muscle of the fetuses

Figure S5. Positive correlation between number of genes showing LOI and bodyweight of LOS fetuses

Figure S6. Verification of the allelic expression of PEG3 and $S N R P N$ in control and LOS fetuses.

Figure S7. Lack of association between allelic expression and transcript abundance of imprinted genes

Figure S8. Allele-specific DNA methylation analyses of $P L A G L 1$ and $N N A T$ DMRs

Figure S9. Differential expression of imprinted genes in LOS compared to controls .79

Table S1. B. t. indicus $\times$ B. t. taurus $\mathrm{F}_{1}$ hybrid fetus information .80 
Table S2. Control and LOS fetal tissues RNAseq summary

Table S3. Allelic expression of the monoallelically expressed imprinted genes in $\sim$ d105 bovine fetuses.

Table S4. Transcript amount and allelic expression of the imprinted genes in

$\sim$ d105 bovine fetuses

Table S5. Imprinted gene expression profile in each tissue of control fetuses .......88

Table S6. Validation of SNPs identified by RNAseq using Sanger sequencing......90

Table S7. Identification of SNPs at DMRs by Sanger sequencing...........................90

Table S8. PCR primer information .91

Chapter 3 Global misregulation of genes largely uncoupled to DNA methylome epimutations characterizes a congenital overgrowth syndrome .92

3.1 Abstract. .02

3.2 Introduction .93

3.3 Results .96

Figure 1. KEGG pathway analyses of DEGs in somatic tissues of LOS fetuses.....98 Figure 2. Multiple pathways involved in myogenesis are misregulated in skeletal muscle of LOS fetuses .101

Figure 3. Genome-wide CpG methylation landscape and its relationship to the transcriptome in day $\sim 105$ skeletal muscle of control fetuses

Figure 4. Identification and characterization of ASM regions in skeletal muscle of control fetuses .106

Figure 5. Identification and characterization of DMRs in fetal skeletal muscle between control and LOS .110

Figure 6. Lack of association between the DEGs and the DMRs in skeletal muscle of the LOS fetuses.

Figure 7. DNA methylation and differential gene expression at IGF2R/AIRN and

$M A G E L 2$ imprinted loci . .114

3.4 Discussion. .115

3.5 Materials and methods .118

3.6 Acknowledgements 
3.7 Supplemental information for Chapter 3

Figure S1. Principle component analyses using all expressed genes in kidney, skeletal muscle, liver, and brain

Figure S2. Identification and characterization of DEGs in the LOS fetuses........130

Figure S3. WGCNA and KEGG pathway analyses in skeletal muscle

Figure S4. Mean CpG methylation level at each WGBS read position of the skeletal muscle from control and LOS fetuses.

Figure S5. Pipeline used for the identification of ASM regions in skeletal muscle of control fetuses

Figure S6. Characterization of ASM regions identified in bovine fetal skeletal muscle 135

Figure S7. Examples of imprinted ASM loci .136

Figure S8. Examples of ASM regions at non-imprinted loci

Figure S9. Mean methylation level differences of the DEGs between control and

LOS \#2 and \#3 at the TSS regions $(-1.5 \mathrm{~kb}$ to $+500 \mathrm{bp})$ .138

Table S1. Summary of the reads alignment and bisulfite conversion rate for each WGBS library .139

Table S2. Reads coverage at $\mathrm{CpG}$ sites

Table S3. ASM regions identified in bovine fetal skeletal muscle using WGBS ..140 Table S4. Methylation status of known imprinted DMRS in human and/or mouse in bovine fetal skeletal muscle .142

Chapter 4 Global assessment of imprinted gene expression in the bovine conceptus by next generation sequencing 143

4.1 Abstract .143

4.2 Introduction .144

4.3 Results

Figure 1. Summary of the pipeline used in this study .149

Figure 2. Identification of adenosine-to-inosine (A-to-I) RNA editing sites in day $\sim 105$ B. t. indicus $\times$ B. t. taurus $F_{1}$ conceptuses .152 
Figure 3. Validation of the allele-specific gene expression in $G N A S / G N A S X L$ and PEG3/USP29 imprinted clusters in day $\sim 105$ B. t. indicus $\times$ B. t. taurus $\mathrm{F}_{1}$ conceptuses

Figure 4. Corroboration of the allele-specificity in gene expression identified by

RNAseq

Figure 5. Summary of the imprinted and monoallelically expressed genes identified in the current study.

Figure 6. Allele-specific expression analyses for autosomal and $\mathrm{X}$-linked genes in placental membranes from day $\sim 105 B$. t. indicus $\times$ B. t. taurus $F_{1}$ hybrids .166

4.4 Discussion .168

4.5 Materials and methods .173

4.6 Acknowledgements . .184

4.7 Supplemental information for Chapter 4 .185

Figure S1. Genotyping the B. t. indicus sire using whole genome sequencing......185 Figure S2. Genotyping the B. t. indicus $\times$ B. t. taurus $\mathrm{F}_{1}$ hybrids using RNAseq .186 Figure S3. Validation of the identified A-to-I RNA editing sites in the B. t. indicus $\times$ B. t. taurus $\mathrm{F}_{1}$ hybrids by Sanger sequencing

Figure S4. Pie charts for the A-to-I RNA editing sites at different gene sections in each tissue of the B. t. indicus $\times$ B. t. taurus $\mathrm{F}_{1}$ hybrids 188

Figure S5. Illustration of the strategy used to assign parental alleles of RNAseq reads in the $B$. t. indicus $\times$ B. t. taurus $\mathrm{F}_{1}$ hybrids

Figure S6. Allele-specific gene expression and DNA methylation analyses at $G N A S / G N A S X L$ imprinted domain in the B. t. indicus $\times$ B. t. taurus $\mathrm{F}_{1}$ hybrids .190 Figure S7. Validation of the allele-specific expression of the genes that are previously described as imprinted in human and/or mouse in the B. t. indicus $\times B$.

t. taurus $\mathrm{F}_{1}$ hybrids

Figure S8. Validation of the allele-specific expression in PEG3/USP29 imprinted domain in the $B$. $t$. indicus $\times$ B. t. taurus $\mathrm{F}_{1}$ hybrids. .192

Figure S9. Validation of the allele-specific expression in $S N R P N$ imprinted domain in the B. t. indicus $\times$ B. t. taurus $\mathrm{F}_{1}$ hybrids 
Figure S10. Corroboration of the allele-specific expression of the singleton genes identified in the B. t. indicus $\times$ B. t. taurus $\mathrm{F}_{1}$ hybrids and the B. t. taurus $\times$ B. $t$. taurus conceptuses

Figure S11. Corroboration of the allele-specific expression of the genes in the $B . t$. taurus $\times$ B. t. taurus conceptuses that are either known imprinted genes in human/mouse or are located in known imprinting clusters. 198

Figure S12. Allelic expression analyses of XIST

Table 1. A-to-I RNA editing sites in bovine that are conserved in human and/or mouse. .154

Table S1. Day $\sim 105$ B. t. indicus $\times$ B. t. taurus $F_{1}$ hybrids RNAseq summary ......203 Table S2. List of A-to-I RNA editing sites identified by RNAseq in the B. $t$. indicus $\times$ B. t. taurus $\mathrm{F}_{1}$ conceptuses ..$*$

Table S3. Allele-specific read counts of monoallelically expressed genes identified by RNAseq in the B. t. indicus $\times$ B. $t$. taurus $\mathrm{F}_{1}$ conceptuses..................................** Table S4. Allelic gene expression in each B. t. indicus $\times$ B. t. taurus $F_{1}$ conceptus Table S5. List of monoallelically expressed genes identified by RNAseq in the $B . t$. indicus $\times$ B. t. taurus $\mathrm{F}_{1}$ conceptus..........................................................................** Table S6. Allele-specific read counts for XIST, X-linked genes, and genes in pseudoautosomal region

Chapter 5 General discussion .205

5.1 How does the use of assisted reproductive technologies lead to epigenetic stability?

5.1.1 Superovulation and loss-of-imprinting .207

5.1.2 Embryos culture and loss-of-imprinting. 208

5.1.3 Loss-of-imprinting in placenta induced by assisted reproductive technologies .209

5.2 Molecular etiology of large offspring syndrome. .210

5.2.1 Loss-of-imprinting in large offspring syndrome .210

5.2.2 Aberrant gene expression at non-imprinted loci in large offspring syndrome 213 
5.2.3 Lack of association between differentially methylated regions and differentially expressed genes in large offspring syndrome ....................................................214

5.2.4 Challenges for studying large offspring syndrome......................................214

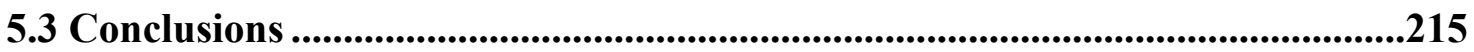

Bibliography ……........................................................................................................217

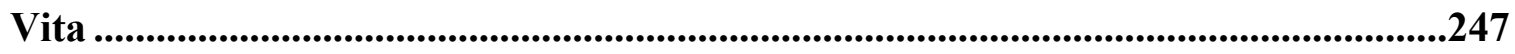

*Due to space limit, Table S2-S5 can only be accessed through the web links, which are directed to the online Epigenetics paper

(http://www.tandfonline.com/doi/full/10.1080/15592294.2016.1184805). 


\section{Preface}

Beckwith-Wiedemann syndrome (BWS) is a congenital overgrowth condition with increased likelihood to develop childhood tumors. Children conceived with the use of assisted reproductive technologies (ART) have an increased frequency to have BWS compared to naturally conceived individuals. In ruminants, the use of ART can induce a similar overgrowth condition that phenotypically recapitulates BWS, which is referred to as large offspring syndrome (LOS). Previously we have shown that LOS is both phenotypically and epigenetically similar to BWS. The overall goal of our research is to use bovine LOS as an animal model to understand the molecular etiology of congenital overgrowth syndromes. As a step to achieve the overall goal, the current project is to comprehensively characterize the bovine LOS at the molecular level.

In this dissertation, chapter 1 reviews the mechanisms of genomic imprinting and describes the current progress in understanding the etiology of the loss-of-imprinting conditions including BWS and LOS. In chapter 2, we determined the allelic expression of imprinted genes previously identified in human and/mouse in bovine control and LOS fetuses. We demonstrated that LOS is a multi-locus-loss-imprinting syndrome, which is similar to what has been reported for a subset of BWS individuals. This study was published in Proceedings of the National Academy of Sciences (PMID: 25825726). In chapter 3, we showed global misregulation of non-imprinted genes in LOS, indicating that loss-of-imprinting is not the only molecular lesion of LOS. Further, most differentially expressed genes in LOS cannot be directly associated with the aberrant DNA methylation, which highlights that caution should be exercised when interpreting time-point-specific DNA methylation data. The manuscript of this study is currently 
under peer review. In chapter 4, for the purpose of de novo identification of novel imprinted genes, we determined global allele-specific gene expression in bovine conceptuses. We identified eight novel imprinted genes in bovine and demonstrated that monoallelic gene expression can be the result of cis-eQTL effect. Further, we expanded the potential adenosine-to-inosine RNA editing sites from $\sim 20$ to 671 in bovine. This chapter was published in the Landes Bioscience Journal: Epigenetics (PMID: 27245094). Lastly, chapter 5 discusses the key questions that remain to be addressed in the field and concludes the dissertation. 


\section{Nomenclature}

ART

AS

ASM

A-to-I RNA editing

$\mathrm{bp}$

B. t. indicus

B. t. taurus

BWS

cDNA

CDS

CPM

CTCF

DEG

DMR

Dnmt

E \#

$5 \mathrm{caC}$

$5 \mathrm{fC}$

$5 \mathrm{hmC}$

$5 \mathrm{mC}$

gDMR

$\mathrm{HCP}$

ICP

ICR

ICSI

IGV

IVC

IVF

$\mathrm{Kb}$
Assisted reproductive technologies

Angelman syndrome

Allele-specific DNA methylation

Adenosine-to-Inosine RNA editing

Base pair

Bos taurus indicus

Bos taurus taurus

Beckwith-Wiedemann syndrome

Complementary DNA

Coding sequences

Counts per million

"CCCTC" binding factor

Differentially expressed gene

Differentially methylated region

DNA methyltransferase

Embryonic day \#

5' carboxylcytosine

5' formylcytosine

5 ' hydroxymethylcytosine

5' methylcytosine

Gametic differentially methylated region

High CpG density promoter

Intermediate $\mathrm{CpG}$ density promoter

Imprinting control region

Intracytoplasmic sperm injection

Integrative Genomic Viewer

in vitro culture

in vitro fertilization

Kilo base pairs 
KEGG

LCP

LOI

LOS

$\mathrm{Mb}$

ncRNA

PAGE

PCA

PCR

PGC

PWS

RDD

RFLP

RPKM

RT

SCNT

SNP

SRS

TES

TSS

WGBS

WGCNA
Kyoto Encyclopedia of Genes and Genomes

Low $\mathrm{CpG}$ density promoter

Loss-of-imprinting

Large offspring syndrome

Mega base pairs

Non-coding RNA

Polyacrylamide gel electrophoresis

Principle component analysis

Polymerase chain reaction

Primordial germ cell

Prader-Willi syndrome

RNA-DNA differences

Restriction fragment length polymorphism

Reads per kilobase of transcript per million mapped reads

Reverse transcription

Somatic cell nuclear transfer

Single nucleotide polymorphism

Silver-Russell syndrome

Transcription end site

Transcription start site

Whole genome bisulfite sequencing

Weighted gene correlation network analyses 


\section{Chapter 1 Literature review}

\subsection{General overview of genomic imprinting}

Genomic imprinting is a series of precisely regulated epigenetic processes that lead to parental-allele-specific expression of a subset of genes in mammals (reviewed in Bartolomei and Ferguson-Smith, 2011). Since the discovery of the first three imprinted genes, namely, Igf2, Igf2r and $H 19$ in 1991 (Barlow et al., 1991; Bartolomei et al., 1991;

DeChiara et al., 1991), more than 100 genes have been identified to be imprinted in mice and humans (http://www.mousebook.org/mousebook-catalogs/imprinting-resource; http://www.geneimprint.com/). Most of the identified imprinted genes are located in clusters with two or more imprinted genes that spread over $20 \mathrm{~kb}$ to $3.7 \mathrm{Mb}$ of DNA (reviewed in Edwards and Ferguson-Smith, 2007). Within each cluster, a discrete region of DNA, termed as imprinting control region (ICR), regulates the proper allele-specific expression of imprinted genes (reviewed in Bartolomei and Ferguson-Smith, 2011). ICRs are marked with parental-allele-specific DNA methylation, which distinguishes the parental alleles in the same nucleus (reviewed in Bartolomei and Ferguson-Smith, 2011). The allele-specific DNA methylation at ICRs is established in a sex-specific manner in the germline. In mice, male germ line methylated ICRs are established before birth, while acquisition of the maternal-specific DNA methylation occurs postnatally during oocyte growth. After fertilization, the gametic DNA methylation imprints escape the wave of global DNA demethylation and are maintained in somatic cells throughout development. Further, DNA methylation is erased in primordial germ cells in order to reestablish the imprints appropriately for next generation (reviewed in Bartolomei and 
Ferguson-Smith, 2011). Over the past $\sim 26$ years, much work has been done to elucidate how DNA methylation is erased and acquired in the germ line and how allele-specific DNA methylation at ICRs are retained when global DNA demethylation occurs postfertilization. Currently, it is recognized that highly coordinated epigenetic processes govern DNA methylation at ICRs to ensure parental-specific expression of imprinted genes.

The importance of genomic imprinting in mammalian development was first demonstrated by the pronuclear transplantation experiments, in which androgenetic and gynogenetic mouse embryos resulted in embryonic lethality (Barton et al., 1984; McGrath and Solter, 1984). Androgenetic conceptuses (derived from two paternal pronuclei) had a severely retarded fetus but a well-developed placenta while gynogenetic fetuses (derived from two maternal pronuclei) were smaller than the bi-parental fetuses and had an undeveloped placenta (Barton et al., 1984; McGrath and Solter, 1984). Later it was confirmed that genomic imprinting is the only barrier for viability of the gynogenetic embryos (Kono et al., 2004). In these experiments, alteration of maternal imprinting by oocyte reconstruction using non-grown oocytes that had a deletion of the $H 19$ region (including the ICR and the $H 19$ transcription unit) allowed gynogenetic embryos to develop to term (Kono et al., 2004). Investigations of imprinted gene function also demonstrated the significance of genomic imprinting in mammalian development. For example, mice with maternal transmission of the non-functional allele of $\operatorname{Igf} 2$, a paternally expressed gene, showed normal growth, but paternal transmission of this mutant allele had deficient growth that was similar to $\operatorname{Ig} f 2$ mull mice (DeChiara et al., 1991). In addition to mouse models, the critical roles of imprinted genes in 
development are also evident in human, cow, and sheep. For instance, BeckwithWiedemann syndrome (BWS) in humans and large offspring syndrome (LOS) in ruminants (i.e., cattle and sheep), two overgrowth conditions that share many phenotypic features, are partially associated with the loss of DNA methylation at the maternal ICR of the imprinted KCNQ1 locus (Chen et al., 2013; reviewed in Weksberg et al., 2010). Although less effort has been focused on determining the function of imprinted gene products when compared to the imprinting mechanisms, these genes emerge as important players in numerous biological processes including fetal growth, placental development, neural development, and behavior (reviewed in Plasschaert and Bartolomei, 2014).

In this chapter, the epigenetic properties of imprinted genes are reviewed with major focus on the dynamic regulation of DNA methylation imprints from germ cells to pre-implantation embryos in mice. Further, the functional roles of imprinted genes in mammalian development are reviewed with detailed description of two loss-ofimprinting disorders: BWS in humans and LOS in cattle.

\subsection{Epigenetic properties of imprinted genes}

\subsubsection{Mechanism of imprinted gene regulation}

Imprinted genes are usually found in clusters containing 2 to 12 genes (reviewed in Edwards and Ferguson-Smith, 2007). These clusters can span 20kb to $3.7 \mathrm{Mb}$ of DNA. Although not as common, examples of solitary imprinted genes do exist. A typical imprinting cluster contains maternally and paternally expressed protein-coding genes as 
well as non-coding RNAs (ncRNAs). Within each cluster, the proper allele-specific expression of imprinted genes is regulated by the core cis-element ICR, which exhibits parental-specific epigenetic modifications such as DNA methylation and histone modifications (reviewed in Bartolomei and Ferguson-Smith, 2011). Deletion of the ICRs can lead to loss-of-imprinting of multiple imprinted genes within a cluster, demonstrating the critical role of ICRs in the regulation of imprinted gene expression (Fitzpatrick et al., 2002; Lin et al., 2003; Thorvaldsen et al., 1998; Williamson et al., 2006; Wutz et al., 1997; Yang et al., 1998). There are several mechanisms by which ICRs initiate and maintain the monoallelic expression of the imprinted genes within each cluster. The bestdefined two mechanisms are the insulator model and the ncRNA model (reviewed in Lee and Bartolomei, 2013).

The insulator model is best exemplified by the H19/Igf 2 imprinted domain in mice. In this cluster, Igf 2 is a paternally expressed protein-coding gene, while $H 19$ is a maternally expressed ncRNA (Bartolomei et al., 1991; DeChiara et al., 1991). The ICR is located approximately $2 \mathrm{~kb}$ upstream of the $H 19$ transcription start site and is paternally methylated while maternally unmethylated (Thorvaldsen et al., 1998; Tremblay et al., 1995). On the maternal allele, the insulator protein CTCF binds to the unmethylated ICR and prevents the $\operatorname{Ig} f 2$ promoter from accessing the downstream enhancers (Bell and Felsenfeld, 2000; Hark et al., 2000; Leighton et al., 1995b). Thus, the permissive chromatin conformation allows the maternal $H 19$ to access the enhancers and gets transcribed. On the paternal allele, however, DNA methylation of the ICR prevents binding by CTCF. Absence of the insulator leads to a chromatin conformation in which the Igf2 promoter engages with the downstream enhancers (Bell and Felsenfeld, 2000; 
Hark et al., 2000; Leighton et al., 1995b). Therefore, $\operatorname{Ig} f 2$ is activated on the paternal allele by the downstream enhancers while $H 19$ is repressed. As illustrated by this example, the DNA methylation status of the ICR directs the allelic regulation of imprinted genes.

The other major imprinting mechanism is the ncRNA model, which can be applied to the Kcnq1/Kcnq1otl and Igf2r/Airn imprinted clusters. The promoter of the long ncRNA can serve as the ICR for the imprinted cluster (Fitzpatrick et al., 2002; Mancini-DiNardo et al., 2003; Smilinich et al., 1999; Stoger et al., 1993; Wutz et al., 1997). For the Kcnq1/Kcnq1ot1 imprinted domain the unmethylated promoter/ICR on the paternal allele allows the transcription of the long ncRNA Kcnq1ot1, which can recruit and expand repressive histone modifications to silence the flanking paternally imprinted genes (Pandey et al., 2008; Terranova et al., 2008). However, on the maternal allele, the ICR is methylated and the Kcnq1otl is not activated, allowing the transcription of the proximal maternal genes. For the Igf2r/Airn imprinted cluster, the unmethylated ICR activates the long ncRNA Airn on the paternal allele, which transcribes antisense through the $I g 2 r$ transcription start site and interferes with the interaction between the Igf $2 r$ promoter and the RNA polymerase II (Latos et al., 2012; Sleutels et al., 2002). In contrast, the ICR is methylated on the maternal allele and Airn is silenced by DNA methylation, and this allows the transcription of $\operatorname{Igf} 2 r$ (Latos et al., 2012; Sleutels et al., 2002). Notably, the allelic regulation process initiated by ICRs, also known as "primary imprints", can set up additional epigenetic differences in the imprinting clusters, such as "secondary DMRs" and allele-specific histone modifications (reviewed in Sanli and Feil, 2015). These additional epigenetic differences stably propagate during cell divisions and 
could act as a candidate mechanism for tissue-specific imprinting. For example, the allele-specific histone modifications established by the long ncRNA Kcnq1ot1 at the maternally expressed genes (e.g., Ascl2 and Osbpl5) are lost in embryonic lineages while maintained in extra-embryonic lineages (Lewis et al., 2004; Umlauf et al., 2004), resulting in the placental-specific imprinting of these genes.

\subsubsection{Dynamic regulation of genomic imprints}

\section{Erasure of imprints in primordial germ cells}

In mice, specification of primordial germ cells (PGCs) from the epiblast begins on embryonic day 6.5-7.5 (E6.5-7.5) (Ginsburg et al., 1990; Saitou et al., 2002; Sato et al., 2002). Following specification, PGCs then proliferate and migrate along the hindgut to the genial ridge where they colonize by E11.5 (Molyneaux et al., 2001; Tam and Snow, 1981). Before migration, PGCs exhibit similar global DNA methylation profiles to their somatic cell origins (Guibert et al., 2012; Seisenberger et al., 2012). For instance, the germline-specific genes (e.g., Mvh and Dazl) and the pluripotent genes (e.g., Nanog) are repressed by DNA methylation (Maatouk et al., 2006; Seisenberger et al., 2012). However, by E12.5-13.5, the global DNA methylation is very low and the pluripotent genes and germline-specific genes are activated (Maatouk et al., 2006; Seisenberger et al., 2012). Further, parental imprints are erased and the silenced $\mathrm{X}$ chromosome is reactivated in the process of PGC reprogramming (Hajkova et al., 2002; Monk and McLaren, 1981; Tam et al., 1994).

In mice, it is clear that both passive and active DNA demethylation are involved in this process. Currently, it is recognized that the epigenetic reprogramming in PGCs 
begins with a passive DNA demethylation during E8.0-10.5 and is then followed by a wave of active DNA demethylation from E10.5 to 13.5 (Guibert et al., 2012;

Seisenberger et al., 2012; Yamaguchi et al., 2013a). For the passive DNA demethylation, the 5-methylcytosines $(5 \mathrm{mC})$ are diluted by the rapid proliferation of PGCs $(\sim 12 \mathrm{~h}$ per cell cycle) when the DNA methylation machinery is repressed (Kagiwada et al., 2013). Specifically, the de novo DNA methyltransferases (Dnmt3a/3b) and their cofactor Dnmt31 are silenced in PGCs (Hajkova et al., 2002; Kagiwada et al., 2013; Seki et al., 2005). Further, although the maintenance DNA methyltransferase Dnmt1 is abundant in PGCs, its essential cofactor Uhrf1 (also known as Np95) is excluded from the nucleus (Kagiwada et al., 2013). In addition, in support of the replication-dependent DNA demethylation, hairpin bisulfite sequencing can detect increased incidence of hemimethylated DNA strands in PGCs in E9.5-10.5 (Arand et al., 2012).

Following passive dilution of 5mC, active DNA demethylation occurs in PGCs involving the ten-eleven translocation family of dioxygenases Tet1/Tet2 (Ito et al., 2010; Tahiliani et al., 2009) and the thymine-DNA glycosylase Tdg (He et al., 2011; Shen et al., 2013). In the wave of active demethylation, Tet $1 /$ Tet 2 sequentially oxidize $5 \mathrm{mC}$ to 5 hydroxymethylcytosine $(5 \mathrm{hmC}), 5$-formylcytosine $(5 \mathrm{faC})$, or 5 -carboxycytosine $(5 \mathrm{caC})$ (Ito et al., 2011). In support of these mechanisms, the level of $5 \mathrm{hmC}$ has a temporal increase during E9.5-10.5 accompanied by decreasing 5mC levels, which is then followed by a reduction of $5 \mathrm{hmC}$ levels at E11.5-12.5 (Hackett et al., 2013). The $5 \mathrm{mC}$ derivatives (i.e., $5 \mathrm{faC}$ and $5 \mathrm{caC}$ ) can be either diluted through cell proliferation or targeted by Tdg-mediated base excision repair (He et al., 2011; Shen et al., 2013). This is 
exemplified in $T d g$ mutant E11.5 PGCs where the secondary imprints at Igf2 DMR2 exhibit hypermethylation (Cortellino et al., 2011).

Two-phase DNA demethylation process in PGCs suggests that distinct DNA demethylation mechanisms (i.e., passive and active demethylation) affect different genomic sequences. Indeed, the passive DNA demethylation in the early phase affects the bulk genome, while specific sequences including imprinted genes become fully unmethylated only after the Tet1/2-mediated active demethylation phase (Seisenberger et al., 2012). Notably, Tetl knockout males exhibit hypermethylation of the imprinted genes Peg3, Peg10, Impact, Igf2r, Gnas, Grb10, and Kcnq1ot1 DMRs in both sperm and E13.5 PGCs, demonstrating the important role of Tet1 in erasure of the DNA methylation imprints at the late reprogramming stage in PGCs (Yamaguchi et al., 2013b). However, it should be noted that normal imprint erasure could occur in a fraction of the Tet1/Tet 2 mutant mice progenies (Dawlaty et al., 2013; Yamaguchi et al., 2013b), suggesting that replication-dependent dilution of $5 \mathrm{mC}$ is probably sufficient for DNA methylation reprogramming in PGCs while Tet proteins act as a "safety check" for this process.

\section{Establishment of imprints in gametogenesis}

The re-establishment of DNA methylation in germ cells occurs at different times in development in male and female gonads (reviewed in Stewart et al., 2016). In males, global de novo DNA methylation occurs in prospermatogonia from $\sim$ E14.5 until birth (Davis et al., 1999; Li et al., 2004). However, in females, de novo methylation initiates after follicle activation in the postnatal ovary and is complete in fully-grown oocytes (Lucifero et al., 2002). Notably, the acquisition of DNA methylation imprints in growing 
oocytes is asynchronous for different maternally methylated gametic DMRs (gDMRs) (Lucifero et al., 2004; Obata and Kono, 2002). For example, of the analyzed maternal gDMRs, the Snrpn DMR is the first to acquire DNA methylation, followed by Pegl, Peg3, and Impact DMRs (Lucifero et al., 2004; Obata and Kono, 2002). This asynchronous acquisition of DNA methylation at maternal gDMRs suggests that different factors affect the imprint establishments at different loci. In agreement with this, absence of the Zfp57 (a KRAB zinc finger protein) in oocytes abolishes the acquisition of DNA methylation at Snrpn DMR but not Peg1 and Peg3 DMRs (Li et al., 2008).

Besides the asynchronous acquisition of DNA methylation for different gDMRs, the same gDMR can exhibit different rates of de novo DNA methylation for the maternally and paternally inherited alleles (Davis et al., 2000; Lucifero et al., 2004). For example, in prospermatogonia, $H 19 / \operatorname{Ig} f 2 \mathrm{DMR}$ acquires $5 \mathrm{mC}$ on the paternally inherited allele prior to maternally inherited allele (Lee et al., 2010). This phenomenon suggests that other epigenetic modifications still recognize the parental alleles in PGCs despite both alleles being unmethylated. It has been found that the insulator CTCF binds to the maternal allele in prospermatogonia prior to de novo methylation, potentially delaying the acquisition of methylation on this allele (Lee et al., 2010). Further, the chromatin composition of the parental alleles at $H 19 / \operatorname{Ig} f 2 \mathrm{DMR}$ is different prior to acquisition of DNA methylation with the maternal allele being enriched with H3K4me3 compared to the paternal allele, potentially also contributing to the delayed de novo DNA methylation as this histone modification inhibits the binding of Dnmt3l to the Histone 3 tail (Lee et al., 2010; Ooi et al., 2007). 
The establishment of DNA methylation imprints in both male and female germlines requires the de novo DNA methylation machinery, which consists of the de novo DNA methyltransferases Dnmt3a/3b/3c and the non-catalytic cofactor Dnmt31. Dnmt3a and Dnmt31 are required for both maternal and paternal imprint establishment (Bourc'his et al., 2001; Kaneda et al., 2004; Kato et al., 2007). Female mice with Dnmt3l knockout or conditional knockout of Dnmt3a in oocytes fail to establish DNA methylation for all the maternal gDMRs examined and their progeny die around midgestation largely due to the defects of maternal imprinting (Bourc'his et al., 2001; Kaneda et al., 2004). For males, deficient Dnmt3a or Dnmt31 results in meiotic failure in spermatocyte most likely due to the derepression of retrotransposons, precluding the further analyses of paternal imprints in sperm or offspring (Bourc'his and Bestor, 2004; Kaneda et al., 2004; Webster et al., 2005). However, examination of the spermatogonia from the newborn testes of Dnmt3a conditional knockouts indicates that Dnmt3a is essential for the de novo methylation of H19/Igf2 and Gtl2/Dlk1 DMR, but not Rasgrfl DMR (Kaneda et al., 2004). Unlike Dnmt3a and Dnmt3l mutants, mice with conditional Dnmt3b knockout in germ cells and their offspring show no apparent abnormalities with most imprints correctly established except that the Rasgrfl DMR is moderately affected (Kato et al., 2007). Recently, Dnmt3c, which was previously annotated as a pseudogene, has been identified as a de novo methyltransferase and is required for the methylation of evolutionarily young repetitive elements and the imprint establishment only at Rasgrf1 DMR in male germ cells (Barau et al., 2016). 


\section{Mechanisms of establishment of differential imprints in sperms and oocytes}

Prior to determination of the DNA methylome of sperm and oocytes, it was hypothesized that the imprinted gDMRs are specifically targeted by the de novo DNA methylation machinery. However, recent whole genome wide DNA methylation profiling has led to the reconsideration of this assumption. Specifically, sperm cells have very high levels of global DNA methylation ( $80-90 \%)$ with methylated CpGs being evenly distributed throughout the genome except for a few encountered within unmethylated CpG islands (Kobayashi et al., 2012; Smallwood et al., 2011; Smith et al., 2012). However, oocytes exhibit a lower level of genome wide DNA methylation ( $\sim 30$ $40 \%$ ) with alternating hypermethylated and hypomethylated domains (Kobayashi et al., 2012; Smallwood et al., 2011; Smith et al., 2012). The disparity in global DNA methylation between sperm cells and oocytes led to the discovery of gDMRs beyond the classical imprinted gDMRs (Kobayashi et al., 2012; Smallwood et al., 2011; Smith et al., 2012). Unlike the imprinted gDMRs, the majority of the newly discovered gDMRs are transient and not maintained throughout development (Kobayashi et al., 2012; Smallwood et al., 2011; Smith et al., 2012). Therefore, the imprinted gDMRs are selectively maintained after fertilization (discussed in detail later, page 17) instead of specifically targeted by de novo DNA methylation machinery over non-imprinted gDMRs during gametogenesis. To date, 26 definitive imprinted gDMRs have been identified in mice with 23 being maternally methylated and 3 being paternally methylated (reviewed in Stewart et al., 2016).

Although the mechanism that involves the establishment of different patterns of DNA methylation in sperm cells and oocytes remains elusive, the recent findings 
highlight that the acquisition of DNA methylation, at least in oocytes, is coordinately regulated by transcription and histone modifications (reviewed in Hanna and Kelsey, 2014). The first piece of evidence that connects histone modification and DNA methylation comes from the finding that the specific interaction between the amino terminus of histone $\mathrm{H} 3$ and Dnmt31 is strongly inhibited by methylation at lysine 4 of $\mathrm{H} 3$ (Ooi et al., 2007). Further, it was found that histone H3, but not H3K4me3, can release the catalytic domain of Dnmt3a from the auto-inhibition by its Atrx-Dnmt3-Dnmt31 (ADD) domain and stimulate the enzymatic activity of Dnmt3a (Guo et al., 2015). In agreement with this, DNA methylation in oocytes is strongly anti-correlated with the broad H3K4me3 domain that covers $\sim 22 \%$ of the genome (Dahl et al., 2016). Further, oocytes deficient of Kdm1b (also known as Aof1 or Lsd2), a H3K4 demethylase, fail to establish DNA methylation at hundreds of $\mathrm{CpG}$ islands including several maternal gDMRs such as Mest, Grb10, Zac1 and Impact DMR (Ciccone et al., 2009; Stewart et al., 2015). Most importantly, profiling of $\mathrm{H} 3 \mathrm{~K} 4 \mathrm{me} 2 / 3$ enrichment in primary and growing oocytes indicates that removal of $\mathrm{H} 3 \mathrm{~K} 4 \mathrm{me} 2 / 3$ occurs prior to the onset of de novo DNA methylation (Stewart et al., 2015), suggesting that the methylation machinery is directed by pre-existing chromatin marks in female germ cells. Lastly, H3K4me2/3-enriched regions in male germ cells are resistant to acquiring DNA methylation, highlighting the critical role of $\mathrm{H} 3 \mathrm{~K} 4 \mathrm{me} 2 / 3$ in protection against de novo DNA methylation (Singh et al., 2013).

Besides histone modifications, several lines of evidence indicate that transcription plays a role in the establishment of maternal imprints in oocytes (reviewed in Hanna and Kelsey, 2014). For example, in the Gnas cluster, truncation of a transcript Nesp, which 
is transcriptionally active in oocytes and overlaps with the ICR, leads to the failure of maternal DNA methylation establishment at this locus (Chotalia et al., 2009). In addition, similar results were obtained for the maternal imprinted gDMRs Snrpn and Plagl1 (Smith et al., 2011; Veselovska et al., 2015). Notably, comprehensive profiling of the oocyte transcriptome and DNA methylome indicates that transcription events correlate with $\sim 90 \%$ of the DNA methylation established in the oocyte, including the methylated CpG islands and imprinted gDMRs (Veselovska et al., 2015). Although a small portion of actively transcribed genes escape DNA methylation, it is likely that transcription is a universal mechanism that contributes to the acquisition of DNA methylation at both imprinted and non-imprinted loci in female germ cells (Veselovska et al., 2015).

A question that remains to be answered is how exactly transcription and histone modifications coordinately direct de novo DNA methylation in oocytes. Current understanding is that activation of oocyte transcriptome accumulates $\mathrm{H} 3 \mathrm{~K} 36 \mathrm{me} 3$ at gene bodies, a histone modification that is associated with transcript elongation and can recruit Dnmt3a to target regions (Dhayalan et al., 2010). Further, prior to the onset of de novo methylation, the $\mathrm{H} 3 \mathrm{~K} 4$ methylation at regions destined for DNA methylation has to be removed by Kdm1b, a process that appears to be also dependent on transcription (Stewart et al., 2015). In support of this, truncation of a transcript that overlap with the imprinted Plagll DMR leads to the failure of DNA methylation establishment, which is coupled with the absence of $\mathrm{H} 3 \mathrm{~K} 36 \mathrm{me} 3$ and retention of $\mathrm{H} 3 \mathrm{~K} 4 \mathrm{me} 2$ at the imprinted gDMR (Stewart et al., 2015). Further, RNA-seq profiling of $K d m I b$ mutant oocytes indicates 
that the transcriptome is largely normal, suggesting $\mathrm{Kdm} 1 \mathrm{~b}$ removal of $\mathrm{H} 3 \mathrm{~K} 4$ methylation is downstream of transcription (Stewart et al., 2015).

The Rasgrfl DMR is distinct from the other two paternal imprinted gDMRs because it relies on Dnmt3b and Dnmt3c (Barau et al., 2016; Kato et al., 2007). Dnmt3c is evolved as a duplication of Dnmt $3 b$, and both are responsible for the methylation of retrotransposons (Barau et al., 2016; Kato et al., 2007). In male germ cells, DNA methylation of retrotransposons also relies on a class of small RNA called PIWIinteracting RNAs, or piRNAs (reviewed in Weick and Miska, 2014). piRNA can silence repetitive elements transcriptionally by recruiting machinery for de novo DNA methylation to the target regions through sequence complementarity (Aravin et al., 2008; Carmell et al., 2007; Itou et al., 2015; Kuramochi-Miyagawa et al., 2008). It has been shown that the Rasgrfl DMR contains repetitive elements that can be targeted by piRNAs (Watanabe et al., 2011), suggesting that de novo DNA methylation at the Rasgtfl DMR depends on the piRNA pathway. In agreement with this, disruption of key piRNA pathway components such as Mili, Miwi2, and Mitopld leads to reduction of DNA methylation of Rasgrfl DMR in sperm cells (Watanabe et al., 2011). Although other piRNA dependent gDMRs exist in the male germline, they do not maintain their methylation status after fertilization suggesting that other mechanisms exist to maintain the imprinted Rasgrfl DMR during development (Watanabe et al., 2011).

\section{Maintenance of imprints during pre-implantation development}

Following fertilization, both oocyte- and sperm-derived genomes undergo global DNA demethylation but the demethylation mechanism is distinct between the parental 
genomes (reviewed in Messerschmidt et al., 2014). The maternal genome undergoes replication-dependent passive DNA demethylation as the methylation maintenance enzyme Dnmt1o is excluded from the nucleus (Howell et al., 2001; Inoue et al., 2011; Inoue and Zhang, 2011). Conversely, the DNA demethylation process on the paternal genome occurs before DNA replication, indicating an active mechanism. It was previously suggested that this active DNA demethylation is the result of Tet3-mediated oxidation of 5mC (Gu et al., 2011; Inoue and Zhang, 2011). In support of this, deletion of Tet3 in oocytes leads to undetectable $5 \mathrm{hmC}$ and results in the hypermethylation of pluripotent genes such as Oct4 and Nanog in zygotes, demonstrating the role of Tet3 in active DNA demethylation (Gu et al., 2011). Recently, it was reported that DNA replication is a major contributor of DNA demethylation of both parental genomes, with Tet3 being critical for demethylation only at certain loci (Shen et al., 2014), raising the question whether active demethylation by Tet3 for paternal pronuclei is required for development. Indeed, live offspring can be derived from round spermatid-injected oocytes, in which the paternal DNA is bound by histones and does not undergo active demethylation in zygotes (Polanski et al., 2008). Further, it has been demonstrated that haploinsufficiency, instead of defective paternal $5 \mathrm{mC}$ oxidation, contributes to the developmental defects previously ascribed to lack of maternal Tet3 in the zygote (Inoue et al., 2015). In fact, a similar rate of neonatal sublethality is observed when Tet3 is deleted from either parental germline (Inoue et al., 2015). In addition, reconstructed zygotes with either oxidized or non-oxidized paternal genome can equally develop to term, indicating that the paternal $5 \mathrm{mC}$ oxidation is dispensable for mouse development (Inoue et al., 2015). Thus, Tet3-mediated active demethylation may function as an 
"accelerator" for DNA demethylation, while replication-dependent dilution of $5 \mathrm{mC}$ alone is probably sufficient for the DNA methylation reprogramming purpose in early embryos.

Unlike in PGCs, the imprinted gDMRs and some repetitive elements such as IAP and centromeric repeats can retain their DNA methylation patterns in the wave of global DNA demethylation in early embryos (reviewed in Messerschmidt et al., 2014). How imprinted gDMRs escape the DNA methylation reprogramming is not completely understood, but several factors have been identified to be responsible for the maintenance of the DNA methylation imprints in pre-implantation embryos. In zygotes, it is well defined that the protein Stella (also known as Pgc7 or Dppa) specifically protects the maternal genome from Tet3-mediated oxidation (Nakamura et al., 2007; Nakamura et al., 2012). The Stella protein distinguishes the maternal genome from the paternal genome by interacting with $\mathrm{H} 3 \mathrm{~K} 9 \mathrm{me} 2$, which is enriched in the maternal genome but not paternal pronuclei (Nakamura et al., 2012). Aside from its protection of global maternal genome from active demethylation, Stella is also required to maintain the allele-specific DNA methylation at several maternal gDMRs such as Pegl, Peg3, and Peg10 and paternal gDMRs such as H19/Igf2 and Rasgrf1 DMR (Nakamura et al., 2007). However, not all imprinted gDMRs are equally affected by the deletion of Stella, suggesting that the imprinted gDMRs are differentially enriched with H3K9me2 (Nakamura et al., 2007), or other mechanisms may act in partial redundancy with Stella to maintain DNA methylation imprints in zygotic stage.

The exclusion of Dnmt1 from the nucleus for passive DNA demethylation in preimplantation embryos poses a challenge for the maintenance of DNA methylation imprint (Howell et al., 2001). It has been found that Dnmt1o, the oocyte-specific isoform of 
Dnmt1, is detected mostly in the cytoplasm with transient localization in the nucleus at the eight-cell stage, suggesting its role in maintenance of genomic imprints at this cell cycle (Howell et al., 2001). Indeed, Dnmt1o deficient embryos exhibit loss of DNA methylation at certain imprinted gDMRs including H19/Igf2, Snrpn, and Peg3 (Howell et al., 2001). Further investigation demonstrated that disruption of both oocyte-specific and zygotic Dnmtl completely eliminates the DNA methylation imprints, indicating that Dnmt1 contributes to DNA methylation maintenance at imprinted gDMRs during preimplantation even though most of the proteins are not located in the nucleus (Hirasawa et al., 2008).

The scarce amount of Dnmt1 in the nucleus during pre-implantation development suggests that a non-canonical mechanism attracts Dnmtl to imprinted gDMRs in early embryos in order to maintain the methylation imprints. Zfp57 was the first maternal factor that was identified to target and maintain DNA methylation at imprinted gDMRs in early embryos (Li et al., 2008; Quenneville et al., 2011). Embryos with disruption of both maternal and zygotic Zfp57 die prenatally and exhibit loss of methylation of multiple imprinted gDMRs such as Snrpn ICR, $H 19$ ICR, and Gtl2/Dlkl ICR (Li et al., 2008). Further, as Zfp57 specifically binds to a methylated hexanucleotide, which can be found in most imprinted gDMRs (MacDonald and Mann, 2014; Quenneville et al., 2011), it is believed that Zfp57 selects the imprinted gDMRs out of the thousands $\mathrm{CpG}$ islands with differential DNA methylation between sperm cells and oocytes (reviewed in Hanna and Kelsey, 2014). After binding to imprinted gDMRs, Zfp57 recruits its co-factor Trim28, which is a component of a multifunctional complex containing H3K9 methyltransferase Setdb1, Dnmt1, and Dnmt3a/3b (Messerschmidt et al., 2012; Zuo et 
al., 2012). Therefore, Trim 28 could be a mediator for Zfp57 to recruit Dnmts to the imprinted gDMRs. In support of this, embryos derived from Trim28-deficient oocytes exhibit variable defects with hypomethylation of several maternal and paternal imprinted gDMRs (Messerschmidt et al., 2012). In summary, it is proposed that Zfp57/Trim28mediated recruitment of DNA methylation maintenance machinery to imprinted gDMRs compensates for the scarce amount of Dnmt1 in the nucleus and ensures the faithful maintenance of methylation imprints in pre-implantation embryos (reviewed in Messerschmidt et al., 2014).

\section{Maintenance of unmethylated ICRs in post-implantation development}

Around the time of implantation, parental genomes undergo another wave of genome-wide de novo DNA methylation to establish cell lineage-specific methylation patterns (reviewed in Smith and Meissner, 2013). In this process, the parental genomes, now in the same compartment, are treated equally by the de novo DNA methylation machinery. Therefore, it is important to protect the unmethylated alleles of imprinted gDMRs from acquisition of de novo DNA methylation. For imprinted gDMRs, the unmethylated alleles are usually characterized by active chromatin marks including H3K $4 m e 2 / m e 3$, histone modifications that can prevent the interaction of Dnmt3a/31 complex with the $\mathrm{H} 3$ tails (Ooi et al., 2007). Therefore, active chromatin marks on the unmethylated allele could be a general mechanism that protects against de novo DNA methylation. Recent findings also indicate that different imprinted DMRs may apply distinct mechanisms to prevent de novo DNA methylation on the unmethylated allele. For H19/Igf2 ICR, ablation of the CTCF binding sites leads to hypermethylation of this locus in neonatal mice but not in oocytes and blastocysts (Schoenherr et al., 2003; Szabo 
et al., 2004), indicating that CTCF binding plays a role in protection against de novo methylation on the unmethylated allele post-fertilization. In addition, for $G t l 2 / D l k 1$ gDMR, it has been demonstrated that polycomb repressive complex 2 (PRC2), a complex that catalyzes the repressive chromatin mark $\mathrm{H} 3 \mathrm{~K} 27 \mathrm{me} 3$, is essential to prevent recruitment of Dnmt3a, Dnmt3b, and Dnmt31 to the DMR for de novo DNA methylation in embryonic stem cells (Das et al., 2015).

\subsection{Functional roles of imprinted genes in mammalian development}

\subsubsection{Imprinted gene network and pre- and post-natal development}

The distinct phenotypes of androgenetic and gynogenetic embryos indicate that parental genomes play different roles in embryonic development (Barton et al., 1984) and the discovery of imprinted genes (i.e., $\operatorname{Ig} f 2, \operatorname{Ig} f 2 r$, and $H 19$ ) demonstrated that imprinted genes are responsible for the functional asymmetry of parental alleles (Barlow et al., 1991; Bartolomei et al., 1991; DeChiara et al., 1991). Igf2 is a paternally expressed fetal growth factor (DeChiara et al., 1991). Biallelic expression of Igf2 leads to embryonic overgrowth while its reduction results in growth restriction (DeChiara et al., 1991). Contrary to $\operatorname{Ig} f 2$, its scavenger mannose receptor $\operatorname{Ig} f 2 r$ is maternally expressed and its deletion leads to overgrowth and embryonic death (Barlow et al., 1991; Ludwig et al., 1996). It has been shown that the lethal phenotype of $\operatorname{Ig} f 2 r$ mutants can be rescued when they carry a mutation that abolishes $\operatorname{Ig} f 2$, indicating an antagonistic relationship between Igf2 and Igf2r in regulation of embryonic development (Ludwig et al., 1996). Unlike $\operatorname{Ig} f 2$ and $\operatorname{Ig} f 2 r$, the functional role of the maternally expressed ncRNA $H 19$ is still not 
completely understood. $\mathrm{H} 19$ was first proposed as a tumor suppressor gene (Hao et al., 1993) and the tumor suppressor activity of $H 19$ was suggested to be mediated by its regulation of $\operatorname{Igf} 2$ in cis. However, transgenic experiments indicate that $H 19$ can act as a trans factor to control embryonic growth and Igf2 is not the only target of $H 19$ (Gabory et al., 2009). In support of this, deletion of the $H 19$ gene leads to overgrowth while transgenic mice that overexpress $H 19$ exhibit reduced bodyweight (Gabory et al., 2009; Ripoche et al., 1997). Further, the expression of multiple imprinted genes that are important embryonic growth regulators such as $\operatorname{Ig} f 2, \operatorname{Ig} f 2 r, C d k n 1 c, D l k 1$, and Rtll are altered when $H 19$ is overexpressed or deleted (Gabory et al., 2009). Therefore, it has been proposed that $H 19$ is a regulator for an imprinted gene network in which multiple imprinted genes are coordinately regulated to precisely control embryonic growth (Gabory et al., 2009).

As the $H 19$ gene expresses both a $2.3 \mathrm{~kb}$ ncRNA and a microRNA (miR-675) (Brannan et al., 1990; Cai and Cullen, 2007), it is still not completely clear whether imprinted gene network is regulated by the ncRNA or the miRNA or both. Overexpression of miR-675 in a variety of embryonic cell lines can lead to reduced proliferation (Keniry et al., 2012). However, miR-675 can only be detected with high abundance in placenta, suggesting that only in placenta this miRNA mediates the H19's growth repressive properties (Keniry et al., 2012). Recently, it has been shown that $H 19$ ncRNA controls gene expression of the imprinted gene network through recruiting the methyl-binding protein 1 (Mbd1) (Monnier et al., 2013). Specifically, the H19-Mbd1 complex can bring repressive histone mark H3K9me3 to the DMRs of the Igf2, Pegl, and Slc38a4 loci and regulate the expression of imprinted genes in an allele-specific- 
independent manner (Monnier et al., 2013). However, how H19 ncRNA specifically recognizes the DMR sequences and why only these DMRs are targeted by H19-Mbd1 complex remain to be addressed. In addition to $H 19$, a paternally expressed transcription factor Plagll has also been demonstrated as a critical regulator of an imprinted gene network to control embryonic growth (Arima et al., 2005; Varrault et al., 2006). Paternal deletion of Plagll leads to intrauterine growth restriction and affects embryonic bone development (Varrault et al., 2006). Further, misregulation of Plagll can alter the expression of multiple imprinted genes including Igf2, H19, CdknIc, and DlkI (Varrault et al., 2006). In addition, the Plagl1 protein can directly regulate the Igf2/H19 locus through binding the downstream enhancers shared by $H 19$ and $\operatorname{Ig} f 2$ (Varrault et al., 2006). In summary, the imprinted gene network may serve to fine-tune the expressed allele of imprinted genes for precise control of embryonic growth.

In addition to controlling embryonic growth, the imprinted gene network can also be an important indicator for postnatal obesity (Dalgaard et al., 2016). It has been shown that the isogenic mice with Trim 28 haploinsufficiency (i.e., $\operatorname{Trim} 28^{+/}$) can randomly develop an obese phenotype, which is associated with the reduced expression of an imprinted gene network including Nnat, Peg3, Cdkn1c, and Plagll (Dalgaard et al., 2016). Notably, the fact that no altered DNA methylation is detected at the ICRs of these imprinted genes is consistent with the findings that imprinted gene network regulation is not dependent on alteration of DNA methylation or loss of allele-specific expression of imprinted genes (Al Adhami et al., 2015; Dalgaard et al., 2016; Monnier et al., 2013). 


\subsubsection{Roles of imprinted genes in placenta}

Numerous mouse models with deletion or overexpression of imprinted genes have demonstrated that precise control of genomic imprinting is essential for normal placental development. For instance, embryos with deletion of the maternally expressed Ascl2 fail to develop spongiotrophoblast and cannot survive beyond embryonic day 10.5 (Guillemot et al., 1994). Conversely, another maternally expressed imprinted gene Phlda2, when deleted, causes the expansion of spongiotrophoblast and increased placental weight (Frank et al., 2002). Further, deletion of Sfmbt2 (paternally expressed) leads to reduction of all trophoblast cell types and embryos die before E12.5 (Miri et al., 2013). Notably, a retrotransposon-derived imprinted gene Rtll (paternally expressed) is essential to maintain the fetal capillaries in placenta and both its deletion and its overexpression result in late-fetal and/or neonatal lethality in mice (Sekita et al., 2008). In addition to regulating placental development, imprinted genes can control the nutrient supply to the fetus through the placenta. For example, deletion of $\operatorname{Ig} f 2 \mathrm{P} 0$, a placental-specific isoform of $\operatorname{Ig} f 2$, inhibits the nutrient uptake by placenta and leads to embryo growth retardation (Constancia et al., 2002). Lastly, imprinted gene products may act as endocrine factors and modulate maternal metabolic processes. For instance, the protein product of the paternally expressed Dlkl gene is the source of maternal circulating Dlk1 and pregnancies lacking Dlk1 exhibit compromised lipid metabolism (Cleaton et al., 2016).

\subsubsection{Roles of imprinted genes in behavior}

Many imprinted genes are expressed in the brain and the significance of genomic imprinting in neurodevelopment is featured by the evidence that mis-expression of 
imprinted genes can cause abnormal behaviors. For example, Peg3 null females exhibit defects in maternal care behaviors such as milk release and nest building (Champagne et al., 2009). In addition, Peg3 null newborns show suckling defects (Champagne et al., 2009). Similar to Peg3, females with Pegl deletions also exhibit deficient maternal care behaviors such that they do not respond to the neonates appropriately and do not ingest their extra-embryonic tissues afterbirth (a normal mammalian behavior termed placentophagia) (Lefebvre et al., 1998). Recent work also indicates that deletion of the paternal Grb10 results in social dominance and aggression behaviors while deletion of the maternal Grb10 leads to fetal and placental overgrowth (Garfield et al., 2011). Grb10 is imprinted on both alleles, however, unlike the maternal isoform, the paternal Grb10 transcribes from an alternative promoter and is only expressed in a subset of neurons (Garfield et al., 2011). This indicates distinct physiological and behavioral functions of parental alleles of the Grb10 gene. The other imprinted genes known to control social behavior and communication include Ube3a, Snord116, and Ndn, which are described in detail in the following section titled "human loss-of-imprinting disorders" as these genes in humans are associated with Prader-Willi syndrome and Angelman syndrome.

\subsection{Human loss-of-imprinting disorders}

\subsubsection{Prader-Willi syndrome (PWS) and Angelman syndrome (AS)}

Both PWS (OMIM ID: 176270) and AS (OMIM ID: 105830) are complex neurodevelopmental disorders with an occurrence of 1 in 15,000 to 25,000 live births 
(reviewed in Buiting, 2010). Individuals with PWS are typically characterized by low birth weight and hypotonia with poor suckling at pre-weaning stage and hyperphagia and obesity at post-weaning stage. Other main features observed in PWS include short statue with small hands and feet, hypogonadism, mild intellectual disability, and abnormal behavior such as skin picking. AS, which is also known as "happy puppet syndrome" due to a happy disposition, are also characterized by developmental delay, severe intellectual disability, speech impairment, seizures, hyperactivity and restless behavior (reviewed in Buiting, 2010). Both PWS and AS are associated with the imprinted SNRPN/UBE3A domain at chromosome 15q11-13 region but exhibit distinct genetic/epigenetic alterations (reviewed in Clayton-Smith and Laan, 2003; reviewed in Ohta et al., 1999). Most PWS individuals ( 70\%) have large deletions ( 4Mb) at the SNRPN/UBE3A imprinted locus on the paternally inherited chromosome, which includes multiple paternally expressed protein coding genes (e.g., SNRPN/SNURF, MAGEL2, $N D N$ ) and ncRNAs (e.g., SNORD115 and SNORD116). The other PWS individuals are characterized by either maternal uniparental disomy of the imprinted locus $(\sim 25 \%)$ or epimutations involving the alteration of DNA methylation at the ICR $(\sim 3 \%)$ (reviewed in Ohta et al., 1999). For AS, approximately $\sim 60-70 \%$ patients also have a large deletion at the imprinted $S N P R N / U B E 3 A$ locus, but unlike PWS, the deletion is always on the maternally inherited chromosome (reviewed in Clayton-Smith and Laan, 2003). Further, approximately $10 \%$ of individuals with AS have a mutation in the maternally expressed gene, ubiquitin protein ligase E3A $(U B E 3 A)$ and 1-5\% exhibit either paternal uniparental disomy or imprinting defects at the imprinted domain (reviewed in Clayton-Smith and Laan, 2003). 
It is well established that the $U B E 3 A$ gene is responsible for AS (Kishino et al., 1997; Matsuura et al., 1997). UBE3A is maternally expressed in a subset of neurons while biallelically expressed in peripheral tissues (Yamasaki et al., 2003). Mice that carry a non-functional maternal Ube $3 a$ exhibit many phenotypes that recapitulate AS such as seizures, learning and gait deficit (Jiang et al., 1998; Miura et al., 2002). Further, activation of the normally silent paternal Ube3a in neurons can partially restore the Ube3a protein and alleviate some cognitive defects associated with AS (Meng et al., 2012; Meng et al., 2015). Recently, it has been shown that Ube3a can rescue more symptoms in a mouse model of AS when it is re-expressed at earlier stage (i.e., newborns) compared to a later stage (i.e., juvenile or adulthood), suggesting the importance of timing for Ube $3 a$ expression in normal neurodevelopment (Silva-Santos et al., 2015). In humans, the severity of AS symptoms is associated with different types of mutations in UBE3A with full deletion of chromosome 15q11-13 most severe and point mutation of UBE3A less severe (Gentile et al., 2010; Valente et al., 2013). Unlike AS, PWS is caused by the loss of multiple, rather than a single paternally expressed gene (reviewed in Bervini and Herzog, 2013). Deletion of $N d n$, a paternally expressed gene in the Snrpn/Ube3a imprinted cluster, leads to behavior defects that recapitulate PWS such as skin scraping and modified spatial learning (Muscatelli et al., 2000). However, Ndn null mice do not exhibit obesity, indicating that $N d n$ gene alone does not account for all the symptoms of PWS. Further, deletion of the Snord116 gene cluster can lead to hyperphagia, but none of these mutant mice exhibit obesity (Ding et al., 2008). Therefore, these genetically modified mouse model systems do not recapitulate all PWS phenotypes. 


\subsubsection{Silver-Russell syndrome (SRS)}

SRS (OMIM ID: 180860) is a rare loss-of-imprinting disorder with an estimated frequency of 1 in 300,000 and is mainly characterized by pre- and postnatal growth restriction, triangular shaped face, and skeletal asymmetry (reviewed in Azzi et al., 2014). SRS is associated with the H19/IGF2 imprinted domain on chromosome $11 \mathrm{p} 15.5$. Approximately $35-65 \%$ SRS patients exhibit hypomethylation of the H19/IGF2 ICR and about $10 \%$ have maternal uniparental disomy of the same imprinted locus (reviewed in Abu-Amero et al., 2008). Of the individuals with epimutation at $H 19 / I G F 2$ domain, $\sim 73 \%$ of them also exhibit hypo- or hypermethylation at other imprinted loci, suggesting a general defect in DNA methylation imprint establishment or maintenance in these individuals (Kannenberg et al., 2012). Recently, a mouse model with the endogenous paternal H19/IGF2 ICR replaced by the human orthologous ICR exhibited impaired DNA methylation acquisition at the locus, resembling the growth restriction phenotype of SRS (Hur et al., 2016). Thus, this mouse model could be used to elucidate some molecular mechanisms that underlie SRS (Hur et al., 2016).

\subsubsection{Beckwith-Wiedemann syndrome (BWS)}

BWS (OMIM ID: 130650) is the most common loss-of-imprinting disorder with occurrence of one in 11,000 live births, although this number is likely underestimated (reviewed in Mussa et al., 2016; Weksberg et al., 2010). BWS is equally represented in males and females (reviewed in Pettenati et al., 1986). The primary features of BWS include pre- and postnatal overgrowth, enlarged tongue, ear malformation, and abdominal wall defects such as exomphalos and umbilical hernia. The less common characteristics 
of BWS include hemihyperplasia, adrenocortical cytomegaly, visceromegaly, congenital renal abnormalities, placentomegaly, and neonatal hypoglycemia (reviewed in Weksberg et al., 2010). Individuals with BWS also have an increased frequency to develop childhood tumors including Wilms' tumors of the kidney, hepatoblastoma, rhabdomyosarcoma, neuroblastoma, and adrenocortical carcinoma (reviewed in Choufani et al., 2013).

Most individuals with BWS ( $85 \%)$ are associated with genetic and/or epigenetic alterations at chromosome $11 \mathrm{p} 15.5$ region, which harbors two imprinted clusters, the KCNQ1 locus and the H19/IGF2 locus (reviewed in Choufani et al., 2013).

Hypomethylation of the ICR (i.e., KvDMR1) of the KCNQ1 imprinted domain is the most frequent molecular alteration of BWS and is seen in approximately $50 \%$ BWS cases (reviewed in Choufani et al., 2013). Aberrant DNA hypomethylation of KvDMR1 causes derepression of the maternal ncRNA KCNQ1OT1, which leads to repression of the flanking maternally expressed genes such as CDK1NC (Diaz-Meyer et al., 2003; Fitzpatrick et al., 2002; Smilinich et al., 1999). CDKN1C is a cyclin-dependent kinase inhibitor of G1 cyclin complex that negatively regulates cell proliferation and the maternal CDKN1C mutations accounts for $\sim 10 \%$ of individuals with BWS (reviewed in Choufani et al., 2013). Hypermethylation of the H19/IGF2 ICR leads to increased abundance of the fetal growth factor of $I G F 2$ and is seen in $\sim 5-10 \%$ BWS cases (reviewed in Choufani et al., 2013). It has been reported that a subset of BWS individuals with hypermethylation of the H19/IGF2 ICR has micro-deletions that disrupt the CTCF binding sites at the locus (Beygo et al., 2013; Sparago et al., 2004). In addition, point mutations of OCT4/SOX2 binding sites at the H19/IGF2 ICR have been 
reported in BWS patients (Abi Habib et al., 2014). Lastly, approximately $20 \%$ BWS cases exhibit paternal uniparental disomy of chromosome $11 \mathrm{p} 15.5$ region, which affects both the H19/IGF2 and KCNQ1 imprinted loci (reviewed in Choufani et al., 2013).

Besides the $K C N Q 1$ and $H 19 / I G F 2$ imprinted loci, approximately 22\% BWS patients with KvDMR1 hypomethylation exhibit DNA methylation defects at other imprinted loci such as PLAGll DMR, PEGI DMR, IGF2R DMR, GNAS DMR (Azzi et al., 2009; Bliek et al., 2009; Eggermann et al., 2016). Of these imprinted genes, PLAGL1 can bind to the KvDMR1 and induce the transcription of KCNQ1OT1 in a DNA methylation-dependent manner (Arima et al., 2005). It is still unclear what causes the DNA methylation defects at multiple imprinted loci in BWS patients. It has been reported that two affected BWS siblings with KvDMR1 hypomethylation have a frameshift mutation of the $N L R P 2$ gene, suggesting that $N L R P 2$ is directly or indirectly implicated in the establishment and/or maintenance of DNA methylation in humans (Meyer et al., 2009).

\subsubsection{Mouse models for BWS}

\section{Mouse models with Cdkn1c deletion}

Several mouse models of BWS have been created by deletion of the $C d k n 1 c$ gene. Maternal deletion of $C d k n 1 c$ leads to neonatal lethality and the $C d k n 1 c$ null fetuses have some defects that resemble BWS, such as abdominal wall defects, cleft palate, and adrenal cytomegaly (Yan et al., 1997; Zhang et al., 1997). However, only prenatal overgrowth but not neonatal overgrowth, is observed in the $C d k 1 n c$ null mice (Tunster et al., 2011). It has been suggested that the prenatal overgrowth of $C d k n 1 c$ null mice is 
compromised by the intrauterine nutrient competition between fetuses during late gestation, as these mutants show larger bodyweight when the litter size is small (Tunster et al., 2011). Further, no neonatal lethality has been reported for BWS, suggesting that, unlike the $C d k n 1 c$ null mouse models, BWS patients with $C D K 1 C$ mutation or hypomethylation of KvDMR1 still retain partial Cdkn1c activity (Chiesa et al., 2012).

\section{Mouse model with the overexpression of Igf 2}

$R / H$ mice. In this mouse model, both $\operatorname{Igf} 2 r$ and H19/Igf2 ICR are targeted in order to increase Igf2 activity. "R" refers to the deletion of $I g f 2 r$, which degrades excess $\operatorname{Ig} f 2$ via receptor-mediated endocytosis (Ludwig et al., 1996). " $\mathrm{H}$ " stands for deletion of the maternal $\mathrm{H} 19$ gene region (including the H19/Igf2 ICR), which leads to the de-repression of $\operatorname{Ig} f 2$ from the maternal alleles (Leighton et al., 1995a). On E12.5, approximately 11fold higher level of Igf2 is present in $\mathrm{R} / \mathrm{H}$ mice in serum compared to controls (Eggenschwiler et al., 1997). This mouse model exhibits many BWS characteristics including somatic overgrowth, abdominal wall defects, visceromegaly, skeletal abnormalities, and cleft palate (Eggenschwiler et al., 1997). However, some BWS features such as macroglossia, renal dysplasia, embryonic tumors and adrenal cytomegaly were not observed in this BWS model. For this mouse model, no pups survive beyond E18.5.

Igf2 transgenic mice. In this model, ES cell lines with exogenous $\operatorname{Ig} f 2$ are injected into the cavity of blastocyst to make chimeras (Sun et al., 1997). The $\operatorname{Ig} 2$ overexpressing chimeras died within 24 hours of birth. On E13.0, transgenic mice with $40-50 \%$ chimeraism exhibit $116-131 \%$ overgrowth compared to controls. In addition to 
the somatic overgrowth, this mouse model also has some BWS features including macroglossia, visceromegaly. However, no abdominal wall defects, neonatal hypoglycemia, and hemihypertrophy are seen in this mouse model (Sun et al., 1997).

\section{Mouse model with the loss of imprinting of Igf2 and null mutation of Cdkn1c}

In this mouse model, both the maternal $H 19$ region (including the H19/IGF2 ICR) and the maternal $C d k n 1 c$ are deleted to increase the Igf2 activity and to deplete Cdkn1c, respectively (Caspary et al., 1999). These double mutants exhibit more severe phenotype than each single mutant. For example, no live offspring can be recovered, and the parturition date of the double mutants is advanced by at least one day when compared to Cdknlc null mutants. The double mutants exhibit $20 \%$ increase in bodyweight at E16.517.5, but are indistinguishable from wild type offspring on E18.5. Other BWS characteristics such as macroglossia (122\% of wild type littermates), placentomegaly (190\% of wild type littermates), kidney dysplasia and abdominal wall defects are observed in the double mutant model (Caspary et al., 1999).

In summary, these mouse models for BWS strongly support that imprinted genes are essential for normal embryonic development and provide insights to the mechanisms of imprinted gene regulation. However, these mouse models do not perfectly fulfill the criteria of an animal model for BWS. First, not all the main BWS symptoms are present in each mouse model system. In particular, childhood tumors, which have an increased likelihood to be developed within the five years of age in BWS, are not present in the mouse models. The absence of tumors might be due to embryonic/neonatal lethality of these mouse models, which is also not seen in BWS patients. Second, the BWS 
characteristics observed in these mouse models are induced by genetic manipulations

(i.e., gene knockout), which do not mimic the epimutations naturally occurring in BWS, such as aberrant DNA methylation at the KCNT1 locus, H19/IGF2, and/or other imprinted loci.

\subsection{Assisted reproductive technologies/therapies (ART) and genomic imprinting}

\subsubsection{ART can induce loss-of-imprinting in animals}

In humans, ART refers to a series of procedures that are used to overcome infertility/subfertility in order to help establish a successful pregnancy with the ultimate goal of giving rise to a healthy child. In animal industry, ART is commonly used to facilitate the rapid multiplication of genetically superior animals. Common ART procedures include ovarian hyperstimulation (also known as superovulation), in vitro fertilization (IVF), intracytoplasmic sperm injection (ICSI), in vitro culture (IVC), and embryo transfer. ART manipulations overlap with the critical period for acquisition and maintenance of genomic imprints. For example, the ovarian hyperstimulation coincides with the oocyte-growing phase when the maternal gDMRs are acquiring DNA methylation (Lucifero et al., 2004; Lucifero et al., 2002). Further, IVF and IVC are implemented when preimplantation embryos undergo global DNA demethylation, in which DNA methylation imprints need to be faithfully maintained (reviewed in Messerschmidt et al., 2014). Therefore, ART manipulations take place during the sensitive epigenetic reprogramming period and may lead to epigenome perturbations. 
Indeed, a large body of experiments has demonstrated that ART can induce loss-ofimprinting in mice, sheep, and cattle (reviewed in Denomme and Mann, 2012).

How ART procedure(s) cause loss-of-imprinting is still unclear. However, it is well established that the multiple ART procedures induce more severe loss-of-imprinting when compared to the use of a single ART manipulation. For example, embryo culture can exacerbate the adverse effects of embryo transfer on imprinted gene expression in E9.5 embryo and placenta (Rivera et al., 2008). Further, it has been shown that high dosage hormone hyperstimulation leads to more frequent imprinted methylation defects in mouse blastocysts when compared to the low dosage (Market-Velker et al., 2010). Notably, superovulation interrupts the paternal $H 19$ methylation in blastocysts, suggesting that superovulation not only disrupts acquisition of imprints in growing oocytes, but also affects the maternal-effect gene products required for imprint maintenance in early embryos (Market-Velker et al., 2010).

It is also intriguing that the placenta is more susceptible than the fetus to loss-ofimprinting when the ART procedures are applied (de Waal et al., 2014; de Waal et al., 2015; Mann et al., 2004; Rivera et al., 2008). It was first suggested that severe loss-ofimprinting in the placenta could be due to the outer position of trophectoderm, which is directly exposed to the artificial environment (e.g., petri-dish) (Mann et al., 2004). The trophectoderm gives rise to tissues in the placenta (reviewed in Rossant and Cross, 2001). However, the yolk sac, which is derived from primitive endoderm that resides in the inner space of the blastocyst, also exhibits similar severity of loss-of-imprinting, suggesting that the "position" hypothesis alone cannot explain the differential loss-of-imprinting susceptibility between embryonic and extra-embryonic tissues (Rivera et al., 2008). It 
also remains to be addressed why isogenic mouse embryos and/or placenta generated with the use of ART exhibit variable and stochastic loss-of-imprinting and why the occurrence of epimutation can be detected ubiquitously or in specific tissues in an individual (de Waal et al., 2014; de Waal et al., 2012a). It is likely that Trim28 activity is impaired in embryos generated with the use of ART as the variability of loss-ofimprinting observed in the ART-conceived offspring can also be found in mice lacking the maternal Trim28 (Messerschmidt et al., 2012).

\subsubsection{ART and BWS}

Several studies have suggested that children conceived with the use of ART have a 3- to 9-fold higher likelihood of being affected with the congenital overgrowth condition BWS (DeBaun et al., 2003; Gicquel et al., 2003; Halliday et al., 2004; Lim et al., 2009; Maher et al., 2003). Recently, a meta-analysis of eight epidemiologic studies indicated that ART-conceived children have a 5.2-fold increased likelihood of developing BWS (Vermeiden and Bernardus, 2013). It is possible that the increased incidence of BWS in ART-conceived offspring could result from the infertility/subfertility of the parents. However, the use of ART can lead to at least some of the imprinting errors as ART-induced loss-of-imprinting has been reported in numerous animal models that have no infertility issues (reviewed in Denomme and Mann, 2012). In agreement with this, a human immunodeficiency virus serodiscordant couple that had no infertility problems sought for ART to conceive a child without the risk of HIV and had a BWS baby after the ovarian hyperstimulation and IVF protocols were applied (Kuentz et al., 2011). 


\subsection{Large offspring syndrome}

\subsubsection{Characteristics of large offspring syndrome}

The use of assisted reproduction in farm animals such as bovine and ovine has facilitated the rapid multiplication of genetically superior animals. However, the use of ART procedures can also induce unwanted pregnancy outcomes. For example, manipulations of oocytes or preimplantation embryos can result in unusually large offspring in ruminants with various congenital abnormalities and the overgrowth condition is referred to as large offspring syndrome (LOS) (reviewed in Young et al., 1998). During the late 1980s and 1990s, in vitro embryo culture techniques typically used a medium supplemented with serum or co-cultured with oviduct epithelia cells (reviewed in Young et al., 1998). At the time, a significant portion of the offspring generated with the use of ART exhibited aberrant phenotypic features including large size, large internal organs, abnormal placenta, and excess placental fluids (reviewed in Young et al., 1998). By the early 1990s, it was recognized that the addition of serum to the medium and the somatic cell co-culture system are major contributors to LOS (reviewed in Young et al., 1998). In addition, offspring produced by somatic cell nuclear transfer (SCNT) can be afflicted with LOS (reviewed in Hill, 2014).

The major feature of LOS is the increased birthweight. It is not uncommon for LOS offspring to have twice the mean bodyweight compared to controls (reviewed in Young et al., 1998). The largest ovine LOS fetus reported so far was five times larger than the normal bodyweight (Walker et al., 1996). It should be noted that the extent of overgrowth is highly variable among LOS individuals, which may depend on the 
different ART procedures used, different animal breeds, and/or different embryo handling practices (reviewed in Young et al., 1998). Further, the full siblings from the same culture micro-drop can express different degrees of overgrown phenotype (Sinclair et al., 2000), suggesting that the induction of LOS by the use of ART is a stochastic process. The overgrowth phenotype can be detrimental for both the dam and the offspring. For the dam, the over-sized nature of the fetus may cause difficult delivery; therefore Caesarean section is commonly used for LOS offspring delivery. For the offspring, the newborns tend to experience difficulties to suckle and breathe.

Another feature of LOS is visceromegaly (internal enlargement of organs in the abdomen), and the affected organs usually are liver and heart (Sinclair et al., 1999; Sinclair et al., 2000). Similar enlargement of liver and heart is also a common feature of individuals with BWS (reviewed in Choufani et al., 2010). LOS has also been associated with placental dysfunctions such as polyhydraminos (Sinclair et al., 1999), which is commonly seen in human BWS (reviewed in Choufani et al., 2010). In sheep LOS, the incidence of polyhydraminos is about $36-62 \%$ in embryos generated with the use of ART (Sinclair et al., 1999). In addition, a range of congenital abnormalities can be observed in LOS, including cleft palates, skeletal defects, high level insulin at birth, and hypoglycemia (Garry et al., 1996) (Schmidt et al., 1996).

It is still unclear how ART procedures cause LOS. It was suggested that ruminant blastocysts in culture media supplemented with serum or somatic cells appeared darker due to excess lipid accumulation in the cytoplasm (Ferguson and Leese, 1999; Thompson, 1997). The accumulation of the lipid droplet was considered to be harmful to embryos and disrupt osmotic regulation (Thompson, 1997). Besides the effect of media 
components, the time period of exposure of embryos to the serum/somatic cells appears critical for the induction of LOS. An ovine experiment showed that ovine embryos are more sensitive to the presence of serum in culture medium before rather than after compaction (Rooke et al., 2007). It is also unclear why induction of LOS by ART is variable even though the same in vitro protocol is used in the same laboratory (reviewed in Young et al., 1998). Overall, LOS exhibits unpredictable and broad range characteristics even when similar protocols and medium are used to produce embryos.

\subsubsection{Molecular alterations of LOS.}

To elucidate the potential molecular alterations responsible for LOS, a large body of experiments has been performed to identify differentially expressed genes between in vitro and in vivo-derived embryos (Bertolini et al., 2002; Lazzari et al., 2002; Niemann et al., 2002; Rizos et al., 2002; Wrenzycki et al., 1998; Wrenzycki et al., 2001; Wrenzycki et al., 2002). It has been reported that genes affected by the in vitro procedures are involved in numerous biological processes such as morula compaction/cavitation, metabolism, trophoblastic function and growth factor signaling (reviewed in Wrenzycki et al., 2004). However, pooled embryos are used in these studies and the gene expression levels of the in-vitro group represent the average of the embryos that will develop LOS and those that will be normal, which may not accurately characterize the molecular signatures of LOS.

Because not every fetus in the in vitro group exhibits an overgrowth phenotype, it is more appropriate to compare the gene expression differences between LOS and nonLOS fetuses instead of between the in vivo and in vitro derived fetuses. In an ovine 
study, late gestation (day 125; ovine gestation=145 days) fetuses with bodyweight greater than $5.5 \mathrm{~kg}$ were considered as LOS (bodyweight of the largest in vivo fetus is $4.5 \mathrm{~kg}$ ) and were compared to controls (Young et al., 2001). It was found that LOS calves have lower $I G F 2 R$ mRNA and protein level in the liver and muscle tissues compared to the controls (Young et al., 2001). In a bovine study, it was observed that two LOS calves were characterized by hypomethylation at the KvDMR1 and decreased expression of CDKN1C (Hori et al., 2010), highlighting the similar molecular alteration of KvDMR1 in LOS and BWS, although parental-specific epimutations were not determined in this experiment. Recently, we have determined the allelic DNA methylation and the allelic expression of the imprinted genes in the KCNQ1 and the H19/IGF2 loci in day 105 Bos indicus taurus $\times$ Bos taurus taurus $\mathrm{F}_{1}$ control and LOS fetuses (Chen et al., 2013). We observed biallelic expression of the ncRNA KCNQ1OT1, loss of methylation of KvDMR1 on the maternal allele, and decreased expression of CDKN1C in two out of seven LOS fetuses (Chen et al., 2013). Our study demonstrated that LOS is both phenotypically and epigenetically similar to human BWS (Chen et al., 2013). From that work we proposed to use LOS as an animal model to study the molecular mechanism that underlie BWS (Chen et al., 2013).

\subsection{Rationale}

Most individuals with BWS are associated with genetic and/or epigenetic alterations at the $K C N Q 1$ locus and the $H 19 / I G F 2$ locus, and a subset of BWS individuals with hypomethylation at the $K C N Q 1$ locus also exhibit aberrant DNA methylation at 
other imprinted loci (Azzi et al., 2009; Bliek et al., 2009; Eggermann et al., 2016). While we have previously described the hypomethylation of the $K C N Q 1$ locus in LOS fetuses (Chen et al., 2013), studies have not yet determined the extent of loss-of-imprinting in this overgrowth condition. Due to the fact that LOS is both phenotypically and epigenetically similar to human BWS, we hypothesize that LOS exhibits loss-ofimprinting beyond the $K C N Q 1$ locus. To test the hypothesis, we determined the allelic expression of imprinted genes previously identified in human and/or mouse in somatic tissues of day $~ 105$ bovine control and LOS fetuses using high-throughput RNA sequencing (Chapter 2). In this study, we identified 20 imprinted genes exhibiting monoallelic expression in control fetal tissues and found that approximately half were biallelically expressed in at least one of the tissues analyzed in LOS. Thus, we conclude that LOS is a multi-locus loss-of-imprinting syndrome, which is similar to what has been observed in some BWS individuals (Azzi et al., 2009; Bliek et al., 2009; Eggermann et al., 2016).

Although it is well accepted that loss-of-imprinting can contribute to BWS and LOS, and as such both conditions have been coined "loss-of-imprinting" syndromes, it remains unknown whether aberrant gene expression and DNA methylation occur at nonimprinted loci and to what extent these molecular alterations contribute to the variable phenotypes observed in these conditions. To address these questions, we examined the transcriptome and DNA methylome of somatic tissues of day $\sim 105$ bovine control and LOS fetuses using high-throughput RNA sequencing and whole genome bisulfite sequencing (Chapter 3). We found that global misreulgation of non-imprinted genes in addition to loss-of-imprinting characterizes the ART-induced overgrowth syndrome. 
Further, most identified DEGs could not be directly associated with altered DNA methylation, highlighting that caution should be exercised when making conclusions about the etiology of such syndromes by interpreting time-point-specific DNA methylation data.

Since discovery of the first three imprinted genes $\operatorname{Ig} f 2, \operatorname{Ig} f 2 r$, and $H 19$ in 1991 (Barlow et al., 1991; Bartolomei et al., 1991; DeChiara et al., 1991), more than 100 genes have been identified to be imprinted in mice and humans (http://www.mousebook.org/mousebook-catalogs/imprinting-resource; http://www.geneimprint.com/). Compared to mice and humans, many fewer imprinted genes (a total of 34) have been reported in domestic farm animal species (reviewed in Tian, 2014). Further, most imprinted genes in cow, pig, sheep, and rabbit have been characterized in studies designed to corroborate the allele-specific expression of genes previously described as imprinted in human and/or mouse (Bischoff et al., 2009; Chen et al., 2015; Chen et al., 2013; Robbins et al., 2012; Young et al., 2003; Zaitoun and Khatib, 2008). For the purpose of de novo identification of imprinted genes in bovine, we determined global monoallelic gene expression in fetal and placental tissues of day 105 bovine conceptuses using RNA sequencing (Chapter 4). In this study, we expand the imprinted gene list in bovine and demonstrate that monoallelic gene expression can be the result of cis-eQTL effects (Chen et al., 2016).

The following three chapters are the full texts of three manuscripts that have been published or under review. Chapter 2 was published in 2015 in "PNAS" (PMID: 25825726), Chapter 3 is under review, and Chapter 4 was published in 2016 in "Epigenetics" (PMID:27245094). The text presented is exactly as in the published work. 
For each chapter, the main figures and tables are included in the body of the text while the supplemental figures and tables are included at the end of each chapter immediately after the acknowledgements. 


\section{Chapter 2 Characterization of global loss of imprinting in fetal overgrowth syndrome induced by assisted reproduction}

\subsection{Abstract}

Embryos generated with the use of assisted reproductive technologies (ART) can develop overgrowth syndromes. In ruminants, the condition is referred to as large offspring syndrome (LOS) and exhibits variable phenotypic abnormalities including overgrowth, enlarged tongue, and abdominal wall defects. These characteristics recapitulate those observed in the human loss-of-imprinting (LOI) overgrowth syndrome Beckwith-Wiedemann (BWS). We have recently shown LOI at the KCNQ1 locus in LOS, the most common epimutation in BWS. Although the first case of ART-induced LOS was reported in 1995, studies have not yet determined the extent of LOI in this condition. Here, we determined allele-specific expression of imprinted genes previously identified in human and/or mouse in day $\sim 105$ Bos taurus indicus $\times$ Bos taurus taurus $\mathrm{F}_{1}$ hybrid control and LOS fetuses using RNAseq. Our analysis allowed us to determine the monoallelic expression of 20 genes in tissues of control fetuses. LOS fetuses displayed variable LOI when compared to controls. Biallelic expression of imprinted genes in LOS was associated with tissue-specific hypomethylation of the normally methylated parental allele. In addition, a positive correlation was observed between bodyweight and the number of biallelically expressed imprinted genes in LOS fetuses. Further, not only was there loss of allele-specific expression of imprinted genes in LOS, but we also observed differential transcript amounts of these genes between control and overgrown fetuses. In summary, we characterized previously unidentified imprinted genes in bovine and 
identified misregulation of imprinting at multiple loci in LOS. We conclude that LOS is a multi-locus LOI syndrome, as is BWS.

\subsection{Introduction}

Genomic imprinting is a series of precisely regulated epigenetic processes that lead to parental allele-specific expression of a subset of genes in mammals (Bartolomei and Ferguson-Smith, 2011). Proper allelic expression of imprinted genes plays an important role in embryonic and neonatal growth, placental function, and postnatal behavior (Miyoshi et al., 2006). Allele-specific DNA methylation at discrete regions established during gametogenesis defines the functional asymmetry of parental alleles (Bartolomei and Ferguson-Smith, 2011). These regions, termed as differentially methylated regions (DMRs), are required to regulate the imprinted expression of these genes. DNA methylation of DMRs is erased in primordial germ cells, re-established during gametogenesis, and maintained when the global DNA demethylation occurs during preimplantation development (Bartolomei and Ferguson-Smith, 2011). In addition to DNA methylation, other epigenetic modifications and mechanisms such as ncRNAs and histone post-translational modifications may contribute to parental-allele specific expression of these genes (Bartolomei and Ferguson-Smith, 2011).

Because of the dynamic epigenetic reprogramming that occurs during oocyte growth and preimplantation development (Morgan et al., 2005), environmental perturbations during this time period, such as the use of assisted reproductive technologies (ART), can affect imprint establishment and maintenance (Denomme and 
Mann, 2012). Numerous prospective studies in animals (de Waal et al., 2012a; de Waal et al., 2012b; Market-Velker et al., 2010; Rivera et al., 2008) and retrospective studies in humans (Vermeiden and Bernardus, 2013) have shown that ART can induce improper regulation of genomic imprinting. ART is commonly used in clinics to treat subfertility and infertility, and each year as high as $5.9 \%$ of infants born in developed countries are conceived by the use of these technologies (CDC, 2013; Kupka et al., 2014). In agriculture, ART is also widely used to increase the number of offspring produced from genetically superior individuals in a shortened period of time (IETS, 2013). Multiple reports have indicated that ART-conceived offspring are more likely to develop imprinting disorders such as Beckwith-Wiedemann syndrome (BWS) in human (Halliday et al., 2004; Lim et al., 2009; Vermeiden and Bernardus, 2013) and large offspring syndrome (LOS) in ruminants (Behboodi et al., 1995; Bertolini et al., 2002; Farin and Farin, 1995; Farin et al., 2006).

BWS is the most common pediatric overgrowth syndrome characterized by complex and variable symptoms such as prenatal and postnatal overgrowth, ear creases, macroglossia, umbilical hernia, and predisposition to develop childhood tumors (Weksberg et al., 2010). BWS has an estimated worldwide frequency of 1 in 13,700 live births (Weksberg et al., 2010) with no noted sex bias (Pettenati et al., 1986) and a weighted relative risk of 5.2 in children conceived with the use of ART (Vermeiden and Bernardus, 2013). Most BWS cases are sporadic and are associated with epimutations in human chromosome 11p15.5, a region that harbors KCNQ1 and H19/IGF2 imprinted loci (Weksberg et al., 2010). Approximately 50\% of BWS cases are associated with the loss of methylation at the KvDMR1 (i.e. KCNQ1 locus) and 2-7\% are associated with the 
gain-of-methylation of the DMR at the H19/IGF2 locus (Weksberg et al., 2010). Recent studies have shown that a subset of BWS individuals with hypomethylation at the $K C N Q 1$ locus also exhibited aberrant DNA methylation at other imprinted loci (Azzi et al., 2009; Bliek et al., 2009; Court et al., 2013; Eggermann et al., 2014; Lim et al., 2009; Maeda et al., 2014; Poole et al., 2013; Tee et al., 2013).

LOS in ruminants exhibits a variable combination of anomalies that recapitulate the phenotypes commonly observed in BWS (Young et al., 1998). These anomalies can have detrimental effects on both the dam and offspring including difficult delivery due to the oversized nature of the fetus and the inability of the newborn to suckle and breathe. We have recently reported that phenotypic and epigenetic similarities exist between LOS and BWS, such as macroglossia, macrosomia, and ear malformation, as well as loss of methylation at the KvDMR1 (i.e. KCNQ1 locus) on the maternal allele (Chen et al., 2013). These parallels make LOS an appropriate animal model for the study of BWS and the understanding of the etiology of these overgrowth syndromes (Chen et al., 2013).

The incidence of LOS complications is variable and little is known about the molecular cause(s) of this complex phenotype. Given the variable phenotypes of BWS and LOS and the fact that only $50 \%$ of individuals exhibit LOI at the KCNQ1 locus (Chen et al., 2013; Weksberg et al., 2010), we hypothesize that these overgrowth syndromes exhibit LOI at loci beyond those primarily used for diagnosis of BWS, namely KCNQ1 and H19/IGF2 DMRs.

In this study, we assessed the allelic expression of imprinted genes previously identified in human and/or mouse in somatic tissues of day $\sim 105$ (d105) bovine control 
and LOS fetuses (term $\approx 280$ days). We identified 20 genes exhibiting monoallelic expression in control fetal tissues and found that approximately half were biallelically expressed in at least one tissue in LOS. Furthermore, we observed that biallelic expression of imprinted genes was associated with loss of DNA methylation at DMRs in a tissue-specific manner. Lastly, our data show that misregulation of imprinted genes goes beyond loss of allele-specific expression and provide insights into the variable gene expression observed in this condition.

\subsection{Results}

Control and LOS bovine fetal tissues preparation and RNA sequencing reads processing

In the current study, we used high throughput RNA sequencing (from here on referred to as RNAseq) to determine allelic expression of imprinted genes previously identified in human and/or mouse in somatic tissues of $\sim \mathrm{d} 105$ control and LOS bovine female fetuses (Fig. 1A). Fetuses used in this study were Bos taurus indicus (B. $t$. indicus $) \times$ Bos taurus taurus $\left(\right.$ B. t. taurus) $\mathrm{F}_{1}$ hybrids (Chen et al., 2013). Only females were used in this study to avoid any potential sex-specific effects on gene expression (Ingleby et al., 2014a). On average, control fetuses weighed 405g while LOS fetuses weighed 592g $(\mathrm{P}=0.008$, Fig. S1 and Table S1). Kidney, brain, skeletal muscle (from here on referred to as muscle), and liver from each fetus were used for RNAseq analysis. These tissues were selected as they are representative of the primary germ cell lineages: 
A

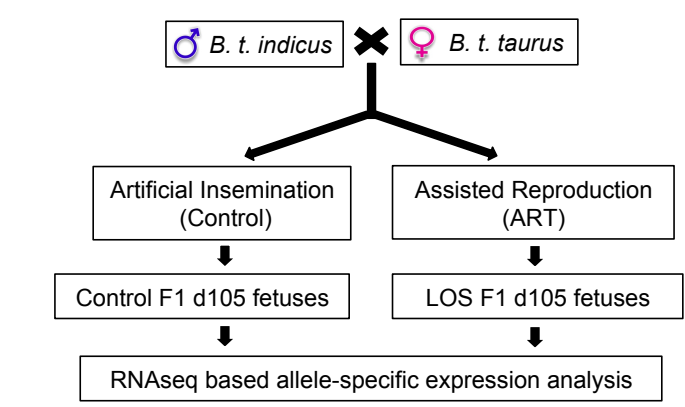

C

\begin{tabular}{|c|c|c|c|c|}
\hline & \multicolumn{4}{|c|}{ LOS (Kidney) } \\
\hline & $\# 1$ & $\# 2$ & $\# 3$ & $\# 4$ \\
\hline BEGAIN & 0 & 0 & 21.3 & 0 \\
\hline DIRAS3 & 0 & 0 & 51.3 & 41.2 \\
\hline$I G F 2 R$ & 17.7 & 15.1 & 32.2 & 21.7 \\
\hline MAGEL2 & 0 & 0 & 0 & 35.6 \\
\hline NAP1L5 & 25.8 & 0 & 0 & 15.5 \\
\hline NNAT & 0 & 0 & 43.1 & 41.8 \\
\hline PEG3 & 0 & 0 & 52.4 & 47.6 \\
\hline PLAGL1 & 0 & 0 & 0 & 35.5 \\
\hline SNRPN & 0 & 0 & 0 & 33.9 \\
\hline & $=-$ & $=-1$ & $=-$ & $=-$ \\
\hline NQ1OT1* & 31.4 & 0 & 0 & 35 \\
\hline
\end{tabular}

B

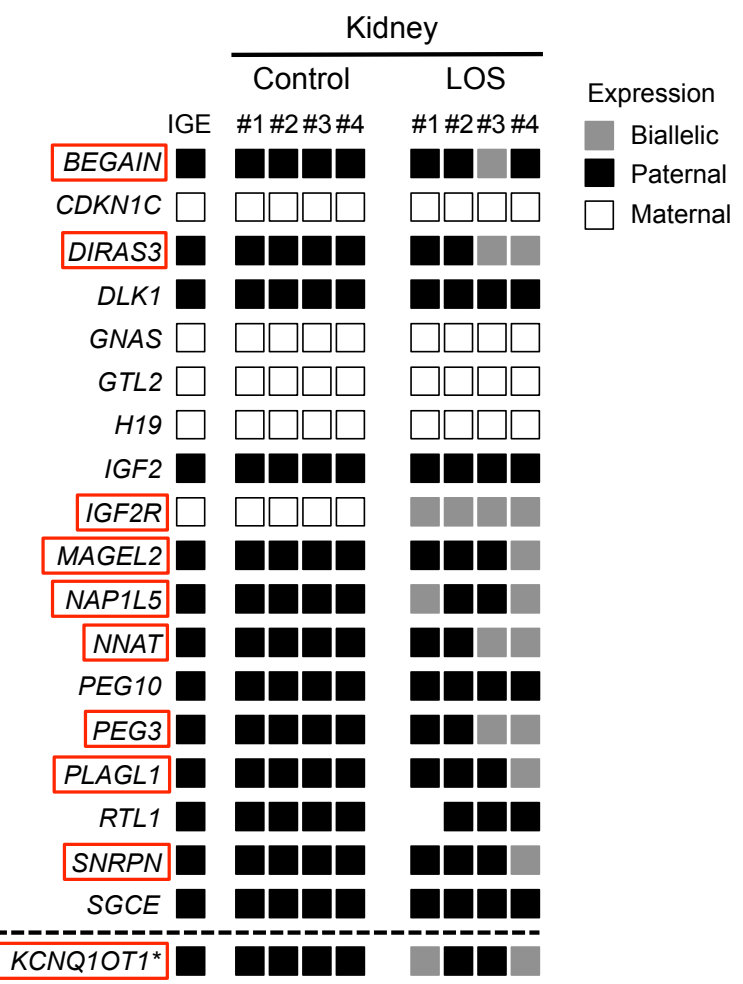

Figure 1. Loss-of-imprinting in large offspring syndrome (LOS). (A) Flowchart of methodology. (B) Allele-specific expression analysis of imprinted genes identified by RNAseq in day $\sim 105$ B. t. indicus $\times$ B. t. taurus fetal kidney. The results for brain, muscle, and liver may be found in Figure S2-4. Gene symbols are ordered from top to bottom in alphabetical order. The parental origin of imprinted gene expression (IGE) is defined according to the known imprinting status in human and/or mouse (maternal = white box; paternal $=$ black box). Samples are arranged from left to right in ascending order by weight (Figure S1) and by treatment group. DNA polymorphisms identified by RNAseq analysis of each $F_{1}$ hybrid were used to discriminate between the parental alleles in control samples in order to establish baseline expression of each gene. A sample with at least $15 \%$ expression from the repressed allele was considered biallelically expressed (grey box). Genes that showed biallelic expression in LOS but not in control fetuses are indicated by red boxes. Missing data for RTL1 indicate lack of discriminating SNPs between parental alleles in the reads. We obtained the allelic expression of the long noncoding RNA KCNQ1OT1 (indicated by asterisk) in a previous study (Chen et al., 2013). (C) Heat map illustrating the degree of loss-of-imprinting for the genes indicated by red boxes in panel (B). Numbers represent the percentage of transcripts expressed from the normally repressed allele. 0 represents $<15 \%$ expression from the repressed allele (actual percentages may be found in Table S3). A summary of the number genes with loss-of-imprinting may be found in Figure S5. 
ectoderm (brain), mesoderm (kidney and muscle) and endoderm (liver). Moreover, kidney and liver are organs that have been documented to be susceptible to tumors in children affected by BWS (Weksberg et al., 2010).

We first aligned the reads to the bovine whole genome assembly B. t. taurus UMD3.1 (Table S2). The reads that aligned to the known imprinted genes were then compared between all four tissues of each fetus and genes with at least one consistent variant nucleotide in at least two tissues were used for subsequent analysis. Of the 105 known imprinted genes detected by RNAseq in our study, 72 genes (Table S4) had at least one single nucleotide polymorphism (SNP) with sufficient reads depth $(\geq 10)$ to assess allelic expression. A gene was considered to be monoallelically expressed if the expression of the allele with the fewer reads accounted for less than $15 \%$ of the total reads. The other 33 genes were not used for further analysis as the heterozygous nature of the bovine makes it impossible for us to determine with any certainty if the reads originated from one or both alleles. For example, if all reads have a base which differs from the reference genome two scenarios are possible; 1) the gene is monoallelically expressed or 2) the gene is biallelically expressed and both alleles contain the same base.

Allelic determination of imprinted genes in $\sim$ d105 control fetal tissues

Of the 72 genes assessable for allelic expression, 52 (Table S4) were biallelically expressed in $\sim \mathrm{d} 105$ fetal kidney, brain, muscle, and liver. The remaining 20 genes were observed to be imprinted in at least one tissue from any fetus. Of these, 18 were expressed monoallelically in the kidney and 14, 17, and 14 in brain, muscle, and liver, respectively (Fig. 1B and Table S3 and Figs. S2-S4). Thirteen genes (i.e. DIRAS3, DLK1, GNAS, GTL2, H19, MAGEL2, NAP1L5, NNAT, PEG3, PEG10, PLAGL1, RTL1, and 
$S N R P N$ ) exhibited monoallelic expression in all the tissues analyzed where the genes were expressed, whereas seven genes (i.e. BEGAIN, CDKN1C, IGF2, IGF2R, INPP5F, PHLDA2, and SGCE) showed tissue-specific imprinting (Fig. 1B and Figs. S2-S4 and Table S3).

Allelic expression of imprinted genes in LOS

Allelic expression of the 20 genes identified to be imprinted in control tissues was then determined in tissues of LOS fetuses. For a gene to be described as experiencing loss-of-imprinted gene expression (as defined by our 15\% cutoff), that gene must be monoallelically expressed in the same tissue in all control fetuses. We observed loss-ofimprinted gene expression in 9, 8, 8, and 6 genes in kidney, brain, muscle and liver, respectively (Fig. 1B, 1C and Figs. S2-S4). Of these, IGF2R showed universal LOI in LOS kidney, muscle, and liver while $I N P P 5 F$ was biallelically expressed in the brain of all LOS fetuses. In other tissues, however, these genes had variable allelic expression in control and/or LOS fetuses (Table S3). Fetuses LOS \#3 and \#4 displayed LOI of NNAT in all the tissues analyzed while LOS \#4 had loss-of-imprinted expression of PLAGL1 in brain, kidney and muscle. Notably, there is a positive correlation between the number of imprinted genes showing LOI in each LOS fetus and their bodyweights (Fig. S5).

\section{Confirmation of SNPS identified by RNAseq}

We first performed Sanger sequencing of genomic DNA from eight B. t. taurus animals, the B.t. indicus sire and several of the B.t.indicus $\times$ B.t.taurus $\mathrm{F}_{1}$ hybrids to verify 37 of the SNPs identified by RNAseq. For 35 SNPs, both the chromosome position and allele variants matched between RNAseq and Sanger sequencing (Table S6). 
The other two SNPs were not confirmed by Sanger sequencing possibly due to RNAseq alignment errors. The confirmed SNPs indicated that SGCE, PEG10, NAP1L5, SNRPN, $P E G 3$, and MAGEL2 were paternally expressed in the $\sim \mathrm{d} 105$ bovine fetal tissues analyzed, showing conservation of maternal-allele silencing of these genes in bovine, human and mouse. We were not able to determine parental allele-specific expression of $N N A T$, since the five SNPs within the exons are heterozygous in both $B$. $t$. indicus and $B$. t. taurus (Fig. 2B and Table S6).

Confirmation of loss-of-imprinted expression identified by RNAseq

To exclude potential false positive imprinted expression caused by RNAseq systemic errors (DeVeale et al., 2012), we verified allele-specific expression of PEG3, NNAT, SNRPN, and PLAGL1 by conventional methods (Fig. 2). Paternal expression was validated for $P E G 3, S N R P N$, and $P L A G L 1$ in control fetuses. In addition, imprinted expression was also confirmed for $N N A T$ although parental origin of the transcript was not assessable due to the lack of subspecies specific polymorphisms (Fig. 2B). In contrast to controls, $P E G 3, S N R P N, N N A T$, and PLAGL1 were biallelically expressed in LOS fetuses (Fig. 2 and Fig. S6).

Association between allelic expression and transcript abundance of imprinted genes

To assess if the biallelic expression of imprinted genes was associated with the increased amount of transcripts, we normalized the read counts aligned to each gene locus to determine the transcript abundance. We found that loss of imprinted gene 
A
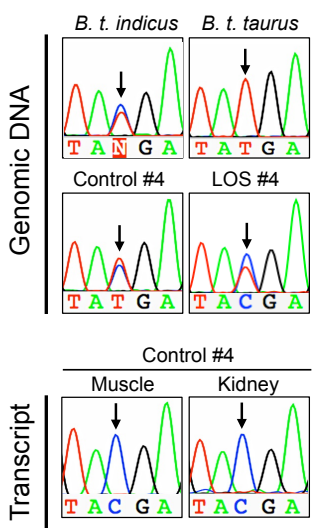

C
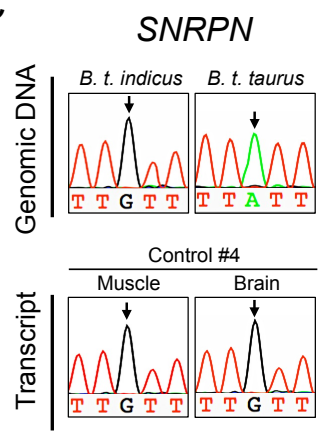

PEG3
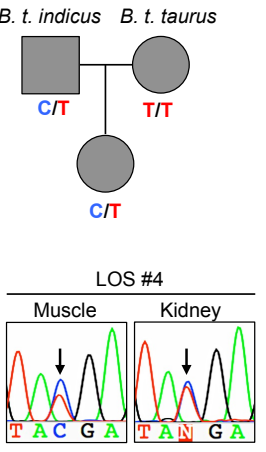

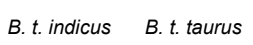
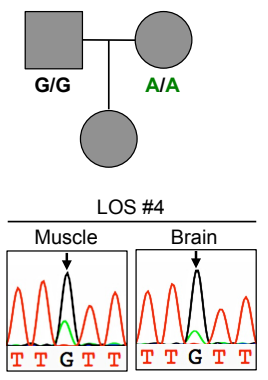

B
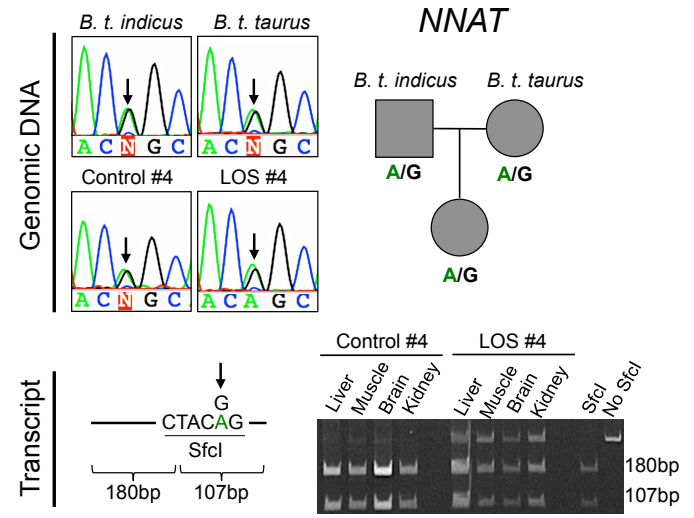

D
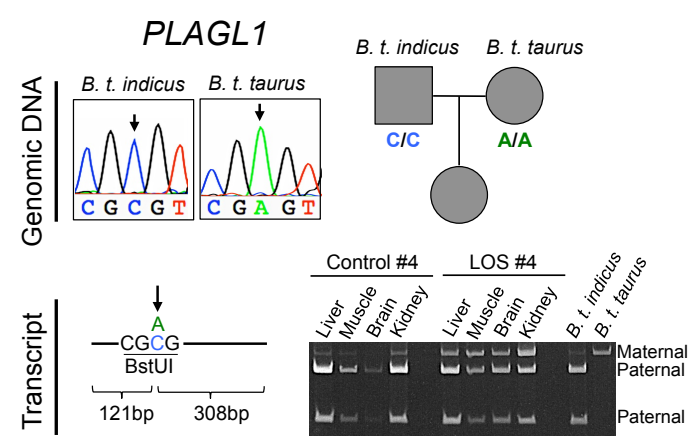

Figure 2. Verification of the allelic expression of PEG3, NNAT, SNRPN, and PLAGL1 in control and LOS fetuses. The top left parts of (A), (B), (C), and (D) are genotyping results obtained by Sanger sequencing of B.t. indicus, B. t. taurus and/or $\mathrm{F}_{1}$ hybrid fetuses for the SNPs identified by RNAseq analysis. SNP positions are indicated by arrows. Pedigree diagrams indicate the SNPs identified by Sanger sequencing for $B$. $t$. indicus (square; i.e. paternal allele in $\mathrm{F}_{1}$ ), B. t. taurus (top circle; maternal allele in the $\mathrm{F}_{1}$ ), and female $\mathrm{F}_{1} \sim \mathrm{d} 105$ fetus (bottom circle). The bottom parts of (A) and (C) are Sanger sequencing results of RT-PCR amplicons of PEG3 and $S N R P N$, respectively. Double peaks demonstrate biallelic expression. The bottom parts of (B), and (D) show allelespecific restriction of RT-PCR amplicons followed by acrylamide gel electrophoresis for NNAT and PLAGL1, respectively. Restriction enzyme (i.e. SfcI for NNAT, and BstUI for $P L A G L 1)$, restriction site and fragment length are illustrated on the bottom left of each panel. 
expression in LOS tissues did not always correlate with the amount of transcript (Fig. S7). For example, increased number of transcripts of $S N R P N, N N A T$ and $P L A G L 1$ were associated with biallelic expression in kidney and muscle $(\mathrm{P}<0.01)$, but not in brain. Further, IGF2R had lower total level of expression in both liver and muscle of all LOS fetuses, although $I G F 2 R$ was biallelically expressed in both tissues.

\section{DNA methylation analysis of PLAGL1, SNRPN, and NNAT DMRS}

As described above, SNRPN, NNAT and PLAGL1 exhibited LOI in several of the LOS fetuses. These genes have also been demonstrated to be associated with BWS (Azzi et al., 2009; Bliek et al., 2009; Court et al., 2013; Tee et al., 2013). Thus we assessed allele-specific DNA methylation of the three DMRs.

DNA methylation of PLAGL1 was examined in a region within the DMR (O'Doherty et al., 2012) that contained $28 \mathrm{CpGs}$ on the paternal (B. t. indicus) and 29 CpGs on the maternal (B.t. taurus) allele (Fig. 3A and Table S7). PLAGL1 is maternally repressed and acquires maternal methylation during oocyte growth in bovine (O'Doherty et al., 2012). As expected, we observed methylated maternal alleles and unmethylated paternal alleles in kidney, brain, muscle, and liver of the control fetus analyzed (Fig. 3A and Fig. S8A). Methylation of CpGs within this region was substantially reduced on the maternal allele in the kidney of LOS \#4 (Fig. 3A) coincident with the biallelic expression of this gene in this tissue (Fig. 1B). However, biallelic expression of PLAGL1 did not coincide with a reduction in DNA methylation in brain and muscle (Fig. S8A).

We next examined the methylation at the DMR (O'Doherty et al., 2012) of the maternally imprinted gene $S N R P N$ (Fig. 3B). The $S N R P N$ DMR displayed differential 
methylation in the brain of the control fetus analyzed (Fig. 3B and Table S7) but showed a substantial loss of methylation in the brain of LOS \#4 fetus. Loss of methylation of this DMR in this tissue (Fig. 3B) is associated with the gain of maternal expression of this gene (Fig. S2).

Lastly, we analyzed DNA methylation at NNAT in control and LOS fetuses. Since the NNAT DMR has not been previously reported in bovine, we chose to analyze a CpG island at the NNAT promoter region (Fig. 3C) that was syntenic to the human NNAT DMR (Hubertus et al., 2013). We examined a region within the $\mathrm{CpG}$ island that contained $33 \mathrm{CpGs}$ on the paternal and $34 \mathrm{CpGs}$ on the maternal allele (Fig. 3C and Table S7). Control fetus \#2 displayed an unmethylated paternal allele and methylated maternal allele in both kidney and brain (Fig. 3C and Fig. S8B), which strongly suggests that NNAT is maternally imprinted in bovine as in human and mouse (Evans et al., 2001; Kagitani et al., 1997). LOS fetus \#4 showed reduced DNA methylation on the maternal allele in kidney but not in brain (Fig. 3C and Fig. S8B).

\section{Determination of the transcript level of imprinted genes previously identified in} human and/or mouse in bovine $\sim 105$ fetuses.

Given the diverse phenotype and various loss-of-imprinted gene expression in LOS, we compared imprinted gene transcript abundance of each LOS fetus to the average of four controls to identify differentially expressed imprinted genes for each LOS individual using edgeR (McCarthy et al., 2012; Robinson et al., 2010). With the threshold of false discovery rate set at $<0.05$ we identified $53,21,47$, and 35 imprinted genes as being differentially expressed in kidney, brain, muscle, and liver, respectively, in at least 
A

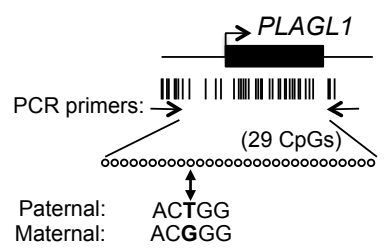

Control \#4 Kidney

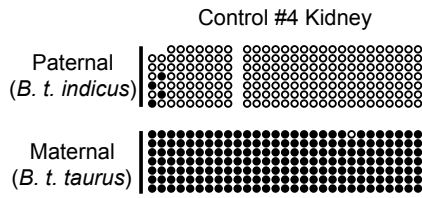

LOS \#4 Kidney
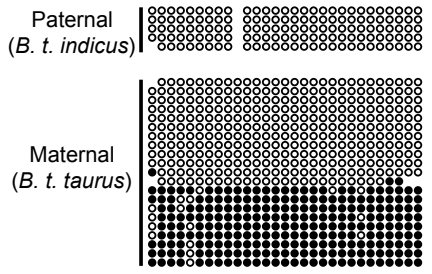

B

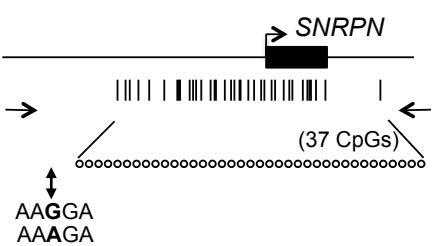

Control \#2 Brain

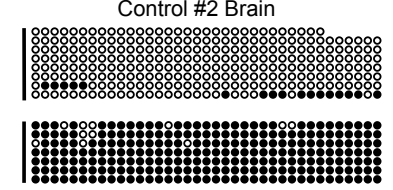

LOS \#4 Brain
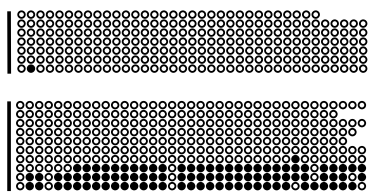

C

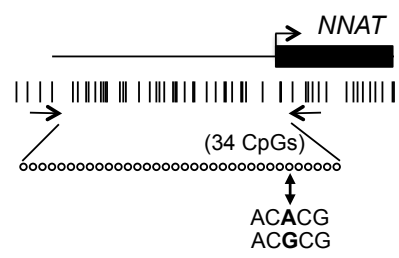

Control \#2 Kidney

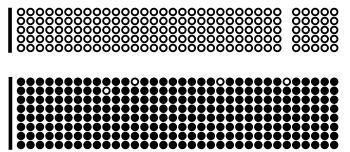

LOS \#4 Kidney
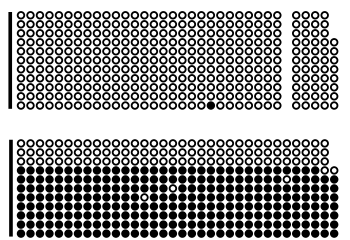

Figure 3. Allele-specific DNA methylation of the PLAGL1, SNRPN, and NNAT DMRs in control and LOS fetuses. The top parts of (A), (B), and (C) are schematic representation of regions analyzed by allele-specific bisulfite sequencing. The bent arrows and black boxes represent the transcription start site and first exon of each gene, respectively. Vertical lines illustrate the $\mathrm{CpGs}$ distributed across the region (drawn to scale). The positions of bisulfiteconverted DNA specific PCR primers are shown as arrows facing each other. Bisulfite map represents of the number of $\mathrm{CpGs}$ contained within each amplicon. Circles represent the CpGs examined within the region of interest (the unmethylated B. t. taurus maternal allele is shown as an example). SNPs (shown in bold) used to determine parental origins are indicated by vertical arrows. The bottom parts of (A), (B), and (C) show the allele-specific bisulfite sequencing of PLAGL1, SNRPN, and NNAT DMRs, respectively. Open circles represent unmethylated CpG sites and filled circle represent methylated $\mathrm{CpG}$ sites. Missing circles are due to SNPs or undetermined bases because of sequencing issues. Each row represents one strand of DNA. 
one LOS fetus (Fig. S9). The fetuses differed in the type of tissue which displayed the highest number of misregulated genes with the kidney of LOS \#3 exhibiting inappropriate levels of expression in 50 of the 53 genes and the muscle of LOS \#4 showing altered expression in 40 of the 47 genes (Fig. S9).

\subsection{Discussion}

Beckwith-Wiedemann syndrome and large offspring syndrome are similar fetal overgrowth conditions (Chen et al., 2013; Kalish et al., 2014). Phenotypically, these are heterogeneous syndromes characterized by variable developmental anomalies including macrosomia, macroglossia, abdominal wall defect, and ear malformations. These syndromes also share LOI at the KCNQ1 locus (Chen et al., 2013), the most common epimutation in BWS, observed in 50\% of patients (Weksberg et al., 2010). Even though reports describing BWS date back to the early 1960's (Weksberg et al., 2005) and the first cases of ART-induced LOS were reported in 1995 (Behboodi et al., 1995; Farin and Farin, 1995), no molecular signature has been identified that can consistently and reliably predict and diagnose these syndromes and/or their varied phenotypes. To date, only a small set of imprinted genes have been queried in LOS (Chen et al., 2013; Hori et al., 2010; Young et al., 2001). With the current study, we advance the field by describing the imprinted signature of $\sim \mathrm{d} 105$ unaffected bovine fetuses and identifying misregulated imprinted loci in LOS.

Approximately 200 imprinted genes have been identified in human and mouse [http://igc.otago.ac.nz/Search.html and http://www.mousebook.org/imprinting-gene-list, 
respectively], and little is known about the allele and tissue specificity of these genes in bovine. In our study we detected expression of 105 of these genes with 52 being biallelically expressed and 20 having monoallelic expression in the fetal tissues analyzed. The remaining 33 genes were not assessable in our system. RNAseq analysis confirmed the maternal expression of $C D K N 1 C, H 19, P H L D A 2$ and the paternal expression of $I G F 2$ and PLAGL1 (Chen et al., 2013; Robbins et al., 2012). In addition, we demonstrate paternal expression of NNAT, NAPIL5, MAGEL2, PEG3,PEG10, SGCE, and SNRPN, which is similar to what has been reported for human and mouse (Morison et al., 2005). Further, we also show that BEGAIN, DIRAS3, DLK1, GNAS, GTL2, IGF2R, INPP5F, and RTL1 are monoallelically expressed, although our analysis precludes us from ascribing the allelic origin of the transcripts. Since monoallelic expression of imprinted genes is tissue- and stage-specific (Bartolomei and Ferguson-Smith, 2011), it is possible that the biallelically expressed genes in the present study are imprinted in other tissues and/or developmental stages. An example is TSSC4 which has been previously reported to be imprinted in bovine placenta (Zaitoun and Khatib, 2008), however, this gene exhibited biallelic expression in fetal $\sim \mathrm{d} 105$ kidney, brain, muscle, and liver.

We demonstrate that half of the imprinted genes in controls displayed biallelic expression in LOS, making this a multi-locus LOI syndrome, as is BWS (Azzi et al., 2009; Bliek et al., 2009; Court et al., 2013; Eggermann et al., 2014; Lim et al., 2009; Maeda et al., 2014; Poole et al., 2013; Tee et al., 2013). The profile of LOI was dependent on the tissue, fetus and degree of macrosomia and may provide insights into the understanding of the etiology of the anomalies observed in LOS and BWS. For instance, Wilm's tumor of the kidney, a high-risk childhood cancer in BWS, displayed 
hypomethylation at the NNAT promoter, which coincided with upregulation of NNAT transcript (Hubertus et al., 2013). In our study we observed a similar situation where biallelic expression and increased transcript amount of $N N A T$ was associated with hypomethylation of its DMR in LOS kidney. Further, we detected biallelic expression of the paternal gene PLAGL1, which corresponded with the increased transcript amount of this gene in the muscle and kidney of the largest LOS fetus. PLAGL1 is a known regulator of embryonic growth and mice lacking the paternal allele of this gene exhibit growth retardation (Varrault et al., 2006). Interestingly, PLAGL1 has been documented to bind the DMR of the KCNQ1 locus in a methylation dependent manner suggesting the involvement of this molecule in the misregulation of this locus, as has been suggested by others (Arima et al., 2005).

In the current study, we also determined the transcript amount of imprinted genes to determine if their misregulation in LOS transcends parental-allele specific expression. Similar to what others have reported in the mouse, biallelic expression of imprinted genes does not always correlate with increased transcript level in bovine (de Waal et al., 2012a; Susiarjo et al., 2013). For example, even though $I G F 2 R$ was biallelically expressed in liver and muscle of LOS fetuses, the total transcript amount was lower than in the controls where monoallelic expression of this gene was observed. Lower IGF2R levels have been previously observed in LOS (Young et al., 2001).

Further, differentially expressed imprinted genes in each LOS fetus were analyzed in the GeneCard database [http://www.genecards.org, (Table 1)] in order to assess the attributes of these genes (e.g. gene ontology, disorders, phenotypes, expression patterns). 
The analysis showed that these genes were most significantly enriched with the descriptors "Beckwith-Wiedemann syndrome", "growth size and body phenotype", "cellular phenotypes", and "tumors" indicating similarities in the types of imprinted genes misregulated in LOS and BWS. In addition, differentially expressed genes were implicated in tumor and/or body size control, which suggests that misregulation of these genes may contribute to the overgrowth phenotype of LOS. The analysis not only allowed us to confirm the imprinted genes previously known to be associated with LOS such as IGF2R (Young et al., 2001) and CDKNIC (Chen et al., 2013), but also to identify new candidates that can be used to predict or diagnose LOS at the molecular level. A gene of interest, for example, is $F B X O 40$, which was downregulated by at least 2 fold in the muscle of all LOS fetuses. Recently, Shi and coworkers demonstrated that FBXO4O is a negative regulator of $I G F 1$ signaling in muscle differentiation and observed increased bodyweight and muscle mass in FBXO40 null mice at six weeks of age (Shi et al., 2011). Other genes with a potential link to the overgrowth seen in LOS are MKRN3 and NNAT which were upregulated in the muscle of the two largest LOS fetuses by $>3.5$ and 2.37 folds, respectively, when compared to controls. MKRN3 and NNAT are protein-coding genes with currently undefined function in muscle.

In summary, our study characterizes previously unknown expression of imprinted genes in unaffected and LOS bovine fetuses. We conclude that LOS is a multi-locus LOI syndrome, as is BWS. Future studies will assess if genes identified in this study are similarly misregulated in BWS. 


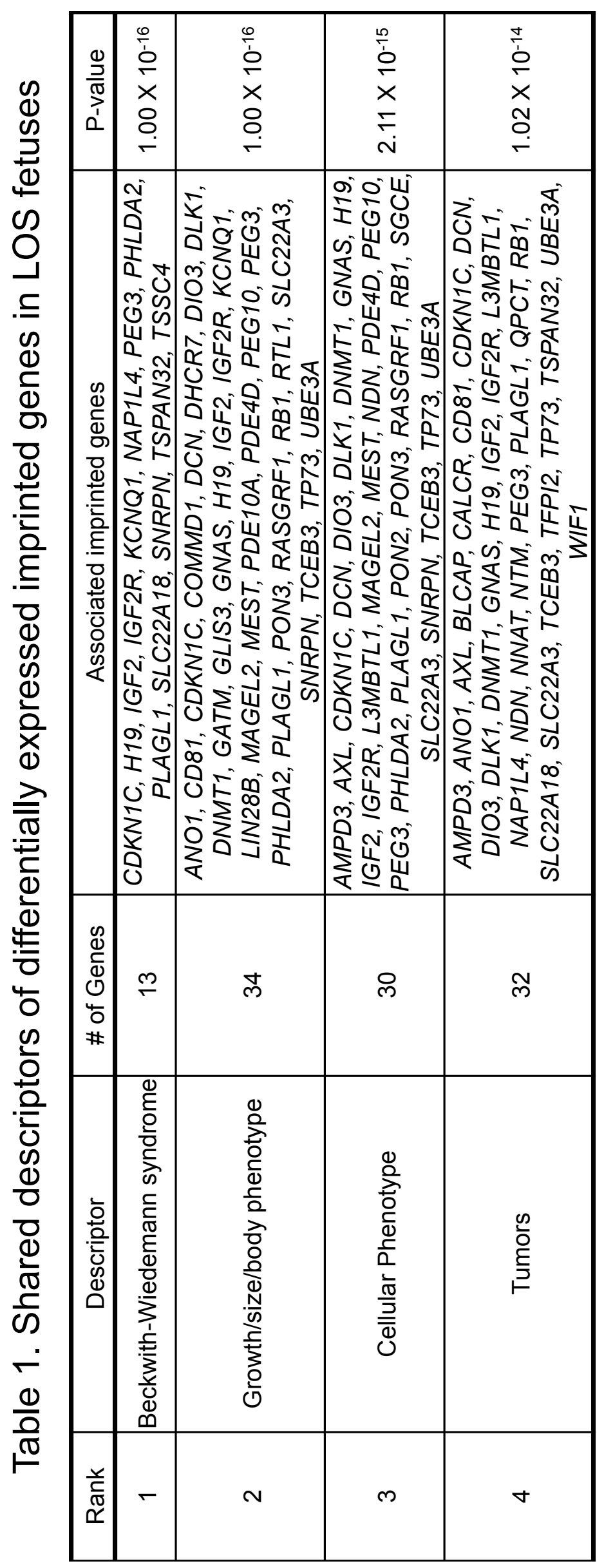




\subsection{Materials and Methods}

Fetal Tissue Collection and Illumina RNAseq

Day $\sim 105$ B. t. indicus X B. t. taurus F1 hybrid control and LOS fetuses were used to analyze allele-specific expression and DNA methylation of imprinted genes. Fetuses used in this study were produced by us as part of a previous study (Chen et al., 2013). Serum was purposely used in that study to supplement the culture media in order to increase the incidence of the overgrowth phenotype. This method enables us to have a reliable system to study the etiology and progression of LOS. In our previous study, we generated 9 controls fetuses ( 5 females and 4 males) and 7 LOS fetuses ( 4 females and 3 males). In the current study, all four females in the LOS group were used. For the control group, we randomly chose four of the five females to analyze the same number of samples in each treatment group.

Total RNA was isolated from kidney, brain, muscle, and liver of four control and four LOS females using Trizol Reagent (Invitrogen) according to manufacturer's instructions. RNA quality was assessed using spectrometry and agarose gel electrophoresis. RNAseq libraries were prepared using standard Illumina protocol and sequenced on Illumina HiSeq 2000 platform as single-end reads. The raw FASTQ files are publically available at Gene Expression Omnibus (GEO; accession no. GSE63509).

\section{Bioinformatics Analysis}

After quality trimming, RNAseq reads were aligned to the bovine genome reference (UMD3.1) using TopHat2 (Kim et al., 2013). Only reads that had $>95 \%$ identity with the reference genome were used for the analyses. Uniquely aligned RNAseq 
reads were normalized to the library size to compute counts per million (CPM). Known imprinted genes were selected based on the human and mouse imprinted gene databases (http://igc.otago.ac.nz/Search.html; http://www.geneimprint.comsite/genes-by-species; http://www.mousebook.org/mousebook-catalogs/imprinting-resource). Imprinted genes annotated by RefSeq or Ensembl were analyzed.

SNPs between B. t. indicus and B. t. taurus were identified by the following criteria: 1) at least 10 reads coverage at a given chromosome position; 2) presence of only one variant allele (different from the reference allele); 3) at least 3 reads for the lower expressed allele. In order to further reduce the false positive SNPs, only the SNPs that exist in at least two of the four tissues (e.g. liver and muscle) from a single individual were kept for further analysis. Only genes that had one or more SNPs and at least 10 SNP-containing read counts were analyzed. Only genes with at least $15 \%$ of reads from the repressed allele were considered biallelically expressed.

\section{SNP validation by Sanger sequencing}

DNA was isolated from B.t.taurus, B.t. indicus, and B.t. indicus $\times$ B.t.taurus $\mathrm{F}_{1}$ hybrid tissues by phenol-chloroform extraction. PCR amplifications were performed using GoTaq Hot Start polymerase (Promega) and amplicons were sequenced at the University of Missouri DNA core using the 96-capillary applied Biosystems 3730 DNA analyzer with Big Dye Terminator.

Analysis of allelic expression

Allele-specific expression of PEG3 and $S N R P N$ were validated using RT-PCR followed by Sanger sequencing. The PCR reactions were prepared and sequenced as 
described above. Allelic expression of NNAT and PLAGL1 were determined using RTPCR followed by RFLP and PAGE.

\section{DNA methylation analysis}

Genomic DNA was mutagenized with sodium bisulfite using the Imprint DNA Modification Kit (Sigma) according to the manufacturer's instructions. Amplified PCR products were ligated with pCC1 vector and cloned using CopyControl PCR cloning kit according to manufacturer's instructions except all the cloning incubation procedures were performed at room temperature. Colonies were usually visible within two days. Positive colonies were sequenced as described above.

\subsection{Acknowledgements}

We thank Dr. Christopher Childers, Colin Diesh, and Dr. Shu Tao for assistance with the RNAseq analysis. We would also like to thank Ms. Kira Marshall for assistance with the manuscript. This work was supported by the National Institutes of Health (grant number 5R21HD062920) and the Food for the 21st Century program at the University of Missouri. 


\subsection{Supplemental information for Chapter 2}

\section{Supplemental material and methods}

Fetal tissue collection

Day 105 control and ART-induced LOS fetuses were generated in a previous study by our laboratory (Chen et al., 2013). Briefly, for the ART group, cumulus-oocyte complexes (COCs) from B. t. taurus (Holstein breed) were matured in vitro and fertilized in vitro using the semen from one $B$. $t$. indicus bull (Nelore breed; ABS CSS MR N OB 425/1677344 29NE0001 97155). Five days post fertilization, embryo culture medium was supplemented with $10 \%$ estrus cow serum. On day 7 post-fertilization, blastocysts were transferred into synchronized B. t. taurus recipients (two blastocysts per recipient; one blastocyst per uterine horn). For control group, B. t. taurus females were artificially inseminated with the semen from the same $B$. $t$. indicus bull used in the ART group. On day $\sim 105$, B. t. indicus $\times$ B. t. taurus $\mathrm{F}_{1}$ hybrid conceptuses (fetus + placental tissues) from both control and ART groups were collected from gravid uteri at caesarean section. For the control group, all fetuses were singleton pregnancies. For the ART group, 11 fetuses were singleton pregnancies and 16 were twins (Chen et al., 2013). Two of the LOS females used here were twins (one fetus/uterine horn; LOS \#1 and LOS \#3) and two were singletons (LOS \#2 and LOS \#4). Statistical analysis showed that bodyweight was not different between twins and singletons $(\mathrm{P}=0.9$ between ART-produced singletons

and twins). At collection, liver, skeletal muscle (from here referred to as muscle), brain, and kidney were well diced, mixed, and snap frozen in liquid nitrogen and stored at $80^{\circ} \mathrm{C}$. All animal surgery procedures were performed at TransOva Genetics (IA) by veterinarians and in accordance with TransOva's animal care and use committee. 


\section{Illumina RNA sequencing (RNAseq)}

Liver, muscle, brain, and kidney RNA samples from four control females and four LOS females were subjected to RNAseq at the University of Missouri-Columbia's DNA core. Total RNA from fetal tissues was isolated using Trizol Reagent (Invitrogen) according to the manufacturer's instructions. RNA quality was determined by both spectrometry and agarose gel electrophoresis. For each sample, $5 \mu \mathrm{g}$ RNA was submitted to the DNA core for RNAseq library preparation using Illumina TruSeq RNA sample preparation kit. Briefly, poly-A containing RNA was purified using magnetic beads with oligo-dT attached. The purified RNA was fragmented and used as a template for cDNA synthesis using random primers. In the following end-repair procedure, an " $\mathrm{A}$ " was added to the 3' end of the cDNA. The cDNA fragments with A-tailing were then ligated with Ttailing adaptors. PCR amplifications of cDNA fragments were followed to generate the final RNAseq libraries.

Initially, liver, muscle, and brain RNAseq libraries from one control fetus (i.e. Control \#2) were sequenced in one lane on Illumina HiSeq 2000 platform to test the sequencing quality and sequencing depth with our RNA samples. We obtained 223.7 million single-end reads (read length $50 \mathrm{bp}$ ) for the three samples. Subsequent RNAseq libraries were sequenced using Illumina HiSeq 2000 platform with single-end read length as $100 \mathrm{bp}$.

\section{RNAseq reads quality trimming}

Raw RNAseq reads were subjected to quality trimming before being aligned to the reference genome. Briefly, adaptor sequences of the reads were removed with the use 
of FastqMcf program (version 1.04.636; ref Aronesty, 2011). Low quality reads (i.e. reads with high percentage of ambiguous base calls) were filtered out using DynamicTrim (version 1.13; ref Cox et al., 2010). DynamicTrim generates the longest contiguous segment of a read in which the phred sequencing score of each base is greater than 20 (base call accuracy $>99 \%$ ). Only reads with a length above $30 \mathrm{bp}$ were kept for further analysis. Lastly, read quality was confirmed by FastQC (version 0.10.1; http://www.bioinformatics.babraham.ac.uk/projects/fastqc/). The number of reads before and after trimming for each sample is summarized in Table S2.

\section{RNAseq reads alignment}

RNAseq reads were aligned to the bovine reference genome assembly UMD3.1 using TopHat2 (version 2.0.10; Kim et al., 2013). By default, TopHat first aligns the reads to the reference genome allowing for a maximum of two mismatches. Reads that span exon-exon junctions would not be aligned and set aside at this step. TopHat then breaks up the exon-exon junction reads into smaller segments and aligns them to the reference genome independently. The aligned smaller segments are then assembled together to generate a final read alignment. The de novo identification of splicing junction sites by TopHat is based on the aligning of exon-exon junction reads. Any two segments pairs that are closer or farther than these parameters are not considered as exon-exon junction reads.

In our analysis, we adjusted the number of allowed mismatches based on read length (i.e. shorter reads were allowed fewer mismatches than longer reads) by passing Bowtie2 scoring option (--score-min L,0,-0.2 --mp 6,6; http://bowtie- 
bio.sourceforge.net/bowtie2/manual.shtml - Scoring-options) through Tophat2 (Kim et al., 2013; Langmead and Salzberg, 2012). This allows us to achieve unique alignment of any read length at greater than $96.7 \%$ identity. In this method, the score for a perfect alignment (i.e. 100\% identity) is 0 while a single nucleotide mismatch within the aligned fragment will be penalized by -6 units. Therefore, the closer the number is to 0 the greater the similarity between the read and the reference genome. For an alignment to be considered valid, then the minimum score cannot be less than $(-0.2) \mathrm{x}$ (read length).

For example; for a 100bp read, the minimum required mapping score is $(-0.2) \mathrm{x}$ $(100)=-20$. Since each mismatch receives a penalty of -6 , then $-20 /-6=3.33$ mismatches allowed. At this level, the alignment identity is $>97 \%$. So using the formula above, the score for a $30 \mathrm{bp}$ read $=-6$ thus only 1 mismatch is allowed for such a read length.

Furthermore, gene and exon models from the Ensembl (http://www.ensembl.org) and the RefSeq (http://www.ncbi.nlm.nih.gov/refseq/) databases were provided to TopHat in order to increase mapping depth at any base. When known gene and exon models are provided, TopHat extracts the transcripts sequences and align the reads to the transcriptome first. This step improves the alignment accuracy of exon-exon junction reads by taking advantage of known splice junctions. The reads that were not aligned to the transcriptome are then aligned to the genome as described above.

Only reads that were uniquely aligned to the reference genome were kept for the subsequent analysis. Percentage of uniquely aligned reads for each sample is summarized in Table S2. 


\section{Transcript assembly from aligned RNAseq reads}

Cufflinks (version 2.1.1; Trapnell et al., 2010) was used to assemble transcripts from the aligned reads for each sample. Assembled transcripts of each sample (i.e. total 32 samples) were merged into a single set of genes and transcripts using Cuffmerge (version 2.1.1; Trapnell et al., 2010) for gene expression analysis. Known gene and exon models from the Ensembl and the RefSeq databases were provided to Cuffmerge to maximize the assembly accuracy. Cuffmerge also performs Cuffcompare (version 2.1.1; Trapnell et al., 2010) to compare the merged transcripts to known gene models. Cuffcompare classifies each transcript into different categories depending on how they match the gene annotation (e.g. class code "=" stands for complete match of a transcript to a known gene intron chain; "u" stands for unknown, intergenic transcript, etc.). Merged transcripts that completely match a known gene intron chain (i.e. class code " $=")$ were assigned with either the Ensembl gene ID or the RefSeq ID if it is not annotated in Ensembl database. Both overall gene expression and allele-specific gene expression were analyzed based on the gene models generated from Cuffmerge.

\section{RNAseq based gene expression analysis}

Gene expression was determined by the total read counts from a gene locus. Total read counts for each gene was generated using the Htseq (version 0.5.4) with default union-counting mode (Anders et al., 2013). In union-counting mode, reads that are located at the overlapping regions of different genes are discarded (Anders et al., 2013). The rationale for this action is that true differential expression of one gene may inaccurately appear as differential expression another gene with overlapped regions. 
Bioconductor package edgeR is a read-counts based RNAseq differential expression analysis tool and determines expression at a gene level based on the total read counts from a gene locus (McCarthy et al., 2012; Robinson et al., 2010). EdgeR normalizes the raw read counts of genes with several parameters (e.g. sequencing depth) excluding gene length (McCarthy et al., 2012; Robinson et al., 2010). Thus edgeR presents gene expression unit as counts per million (CPM) instead of reads per kilobase per million (RPKM) (McCarthy et al., 2012; Robinson et al., 2010). Genes that have less than $10 \mathrm{CPM}$ for the sum of all 8 samples (i.e. control group: $\mathrm{n}=4$ and LOS group: $\mathrm{n}=4$ ) were considered as low expression and not included in the subsequent analysis.

About 100 known imprinted genes in humans and 150 in mouse ( $\sim 50$ overlapped with humans') were obtained from human (http://igc.otago.ac.nz/Search.html; http://www.geneimprint.comsite/genes-by-species) and mouse imprinted gene database (http://www.har.mrc.ac.uk/research/genomic_imprinting/). Approximately 130 known imprinted genes are annotated in bovine Ensembl and/or RefSeq databases and included in Cuffmerge gene models. In the annotated known imprinted gene list, 105 genes were expressed in at least one of the tissues analyzed (i.e. liver, muscle, brain, and kidney) based on the criteria described above.

Gene expression for each tissue from each LOS fetus was compared to the average gene expression of control fetuses. Differential gene expression between each LOS fetus and the average of the controls was identified using edgeR (McCarthy et al., 2012; Robinson et al., 2010). Differentially expressed imprinted genes were uploaded to GeneCards database (http://www.genecards.org/) to identify their shared descriptors. 


\section{RNAseq based allele-specific expression analysis of known imprinted genes}

Single nucleotide polymorphisms (SNPs) between B. t. indicus and B. t. taurus are needed to determine parental-allele specific gene expression. In order to ascertain the allele-specific expression in $\mathrm{F}_{1}$ fetuses, we identified SNPs from the B. t. indicus $\times$ B.t. taurus $\mathrm{F}_{1}$ hybrid fetal RNAseq data.

Reads that were uniquely aligned to the UMD3.1 reference genome (B. t. taurus) were used to identify SNPs. The following criteria were initially applied to identify heterozygous SNPs for each sample: 1) coverage by at least 10 reads at a given chromosome position; 2) presence of only one variant allele (different from the reference allele); 3 ) at least 3 reads for the lower expressed allele. In order to further reduce the false positive SNPs, only the SNPs that exist in at least two of the four tissues (e.g. liver and muscle) from a single individual were kept for further analysis. In addition, SNPs that could not be assigned to a single gene (i.e. a SNP that is located in a region with two different genes with sense and antisense transcription) were not used for allele-specific expression analysis.

With the identified SNPs and SNP-containing read counts, we determined the allele-specific expression of the known imprinted genes by the relative counts of reference and alternative alleles. Only genes that had one or more SNPs and at least 10 SNP-containing read counts were analyzed. 


\section{Determination of Loss-of-imprinting}

Eleven imprinted genes (i.e. PEG3, SNRPN CDKN1C, H19, IGF2, SGCE, PEG10, NAP1L5, PLAGL1, PHLDA2, MAGEL2) were selected to compute the standard deviation of the expression from the repressed allele. These were chosen because Sanger sequencing was performed on this subset of genes as part of validations performed in this and our previous study (Chen et al., 2013) to confirm allelic parental origin.

We assumed that an imprinted gene is monoallelically expressed and hence has no expression from the repressed allele. Therefore, the low level of expression detected from the repressed allele of these genes was categorized as noise, which is expected to follow a normal distribution. We calculated $2 \times \mathrm{SD}$ using all of the aforementioned genes. That number was $\sim 12 \%$. We decided to use the more stringent cutoff of $15 \%$ expression from the repressed allele to ascribe an imprinted gene as being biallelically expressed. Further, we used Pearson correlation to determine associations between allelic expression and transcript amount.

\section{SNPs validation by Sanger sequencing}

DNA was isolated using the phenol-chloroform method. For eight B. t. taurus animals (four Holstein breed and four Angus breed), DNA was isolated from the uterine tissues. DNA of the B. t. indicus bull and B.t. indicus $\times$ B. t. taurus $\mathrm{F}_{1}$ hybrid fetuses was isolated from semen and tails, respectively. PCR primers were designed to amplify 14 genomic regions that contain 37 SNPs identified by RNAseq using IDT primer design program (http://www.idtdna.com/Primerquest/Home/Index). PCR in a $20 \mu \mathrm{l}$ reaction

containing $1 \times$ colorless GoTaq Flexi Buffer (Promega), $0.3 \mu \mathrm{M}$ forward and reverse 
primer (IDT), $2.5 \mathrm{mM} \mathrm{MgCl}_{2}$ (Promega), $200 \mu \mathrm{M} \mathrm{dNTP}$ (Invitrogen), and 0.5U GoTaq Hot Start polymerase (Promega) were under the following cycling program: initial denaturation at $94.0^{\circ} \mathrm{C}$ for 2 minutes 15 seconds, 35 cycles of $94.0^{\circ} \mathrm{C}$ for 15 seconds, 58.4-62. $3^{\circ} \mathrm{C}$ for 30 seconds, and $72.0^{\circ} \mathrm{C}$ for 1 minute, and final extension at $72.0^{\circ} \mathrm{C}$ for 5 minutes (For detailed PCR and primer condition information see Table S8). PCR products were resolved by $1 \%$ agarose gel electrophoresis and products at expected size were excised and cleaned using Wizard DNA Clean Up system (Promega). Cleaned PCR products were sequenced at the University of Missouri DNA core using the 96-capillary applied Biosystems 3730 DNA analyzer with Big Dye Terminator. Sequencing data was aligned to references using MacVector software.

Validation of allele-specific expression of imprinted genes by Sanger sequencing and restriction fragment length polymorphism (RFLP)

RNA was treated with RNase-free DNase (Fisher Scientific) before cDNA synthesis. First strand cDNA synthesis was performed with 80 ng RNA in a $20 \mu$ l reaction; 10mM DTT (Invitrogen), $1 \times$ First Strand Buffer (Invitrogen), 0.5 $\mu$ g random primer (Promega), 1mM dNTP (Invitrogen), 100U Superscript II reverse transcriptase (Invitrogen), and 20U RNase Inhibitor (Fisher Scientific). The cDNA synthesis reaction was first incubated at $42^{\circ} \mathrm{C}$ for 1 hour, followed by $95^{\circ} \mathrm{C}$ for 10 minutes, and stored at $20^{\circ} \mathrm{C}$ until further use. In order to ensure no genomic DNA contamination, a cDNA synthesis reaction without reverse transcriptase (RT) for each sample was also included.

Allele-specific expression of PEG3 and $S N R P N$ were validated using RT-PCR followed by Sanger sequencing (Chen et al., 2013). The PCR reactions were prepared and 
sequenced as described above. Allelic expression of NNAT and PLAGL1 were determined using RT-PCR followed by RFLP and polyacrylamide gel electrophoresis (PAGE). Briefly, SNPs in NNAT and PLAGL1 exons were used to discriminate parental alleles by applying allele-specific restriction enzyme digestion (i.e. SfcI for NNAT, and BstUI for PLAGL1). Detailed PCR and primer information is included in Table S8.

DNA methylation analysis of PLAGL1, SNRPN, and NNAT DMRS

Bisulfite mutagenesis of DNA was conducted using the Imprint DNA Modification Kit (Sigma) according to the manufacturer's instructions. During this process, unmethylated cytosines are converted into uracil, and replaced by thymine during PCR amplifications, whereas methylated cytosines remain cytosines. PLAGL1 DMR (ref|AC_000166.1: 82473331-82474137) and SNRPN DMR (ref|AC_000178.1: 25826-26186) have been reported as gametic DMRs in bovine (O'Doherty et al., 2012). PCR Primers for these regions were designed using the Methyl Primer Express Software (version 1.0, Applied Biosystems). Since NNAT DMR had not been previously reported in bovine, a CpG island (ref|AC_000170.1: 67118285-67119013) at NNAT promoter region identified by MethPrimer (Li and Dahiya, 2002) was used to design primers. All the amplicons cover SNPs between B. t. indicus and B. t. taurus identified by Sanger sequencing. Primer information is included in Table S8.

Bisulfite PCR conditions were similar to those described above except that $4 \mathrm{mM}$ $\mathrm{MgCl}_{2}$ was used. For PLAGL1 DMR, the PCR cycling program was as follows: initial denaturation at $94.0^{\circ} \mathrm{C}$ for 2 minutes 15 seconds, 45 cycles of $94.0^{\circ} \mathrm{C}$ for 30 seconds, $60.5^{\circ} \mathrm{C}$ for 45 seconds, and $72.0^{\circ} \mathrm{C}$ for 1 minute, and final extension at $72.0^{\circ} \mathrm{C}$ for 5 
minutes. For $N N A T$ and $S N R P N$ DMRs, touchdown PCR cycling program was used to optimize the amplification of product. Briefly, after initial denaturation, the annealing temperature was gradually decreased by $1{ }^{\circ} \mathrm{C} /$ cycle for five cycles to the optimized temperature (i.e. $60.5^{\circ} \mathrm{C}$ ). The optimized temperature was used as the annealing temperature for the subsequent 40 cycles.

PCR products were resolved by $1 \%$ agarose gel electrophoresis and isolated as described above. PLAGL1 DMR amplicon (298bp contains 29 CpGs; ref|AC_000166.1: 82473614-82473911), SNRPN DMR amplicon (566bp contains 37 CpGs; ref|AC_000178.1: 25757-26322), and NNAT DMR amplicon (342bp contains 34 CpGs; ref|AC_000170.1: 67118612-67118953) were ligated with pCC1 vector and cloned using CopyControl PCR cloning kit according to manufacturer's instructions except all the cloning incubation procedures were performed at room temperature. Colonies were usually visible within two days. Individual colonies were picked up and diluted with $10 \mu 1$ $\mathrm{H}_{2} \mathrm{O}$, from which $2 \mu \mathrm{lmix}$ was used as template for colony PCR. PCR products were then precipitated with isopropanol. Briefly, $20 \mu \mathrm{l}$ PCR product was added with $4 \mu \mathrm{l} 3 \mathrm{M}$ sodium acetate and $20 \mathrm{ul} 100 \%$ isopropanol and placed at $-80^{\circ} \mathrm{C}$ for at least 15 minutes. After centrifuging at $16,000 \mathrm{~g}$ for 20 minutes, supernatant was removed and pellet (generally invisible) was washed with $70 \%$ ethanol. After repeating the centrifuge step, pellet was suspended in $\mathrm{H}_{2} \mathrm{O}$ and sent for Sanger sequencing as described above. 


\section{Supplemental figures and tables for Chapter 2}

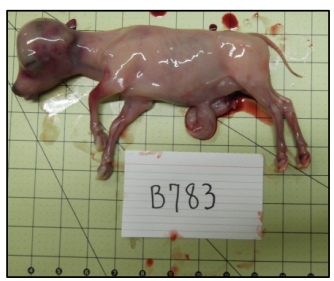

Control \#1: 392g

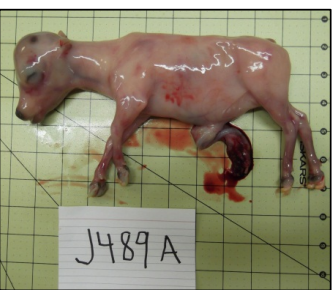

LOS \#1: 514g

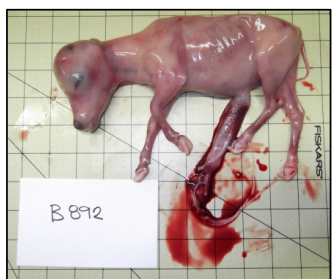

Control \#2: 404g

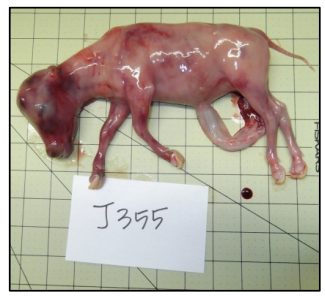

LOS \#2: $518 \mathrm{~g}$

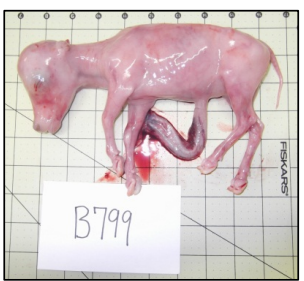

Control \#3: 408g

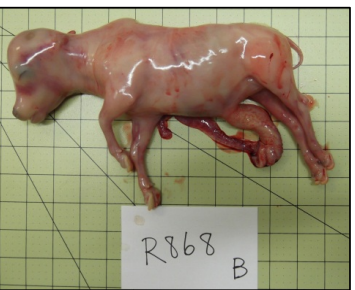

LOS \#3: 620g

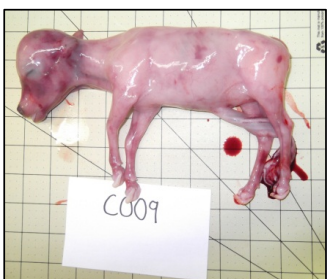

Control \#4: 416g

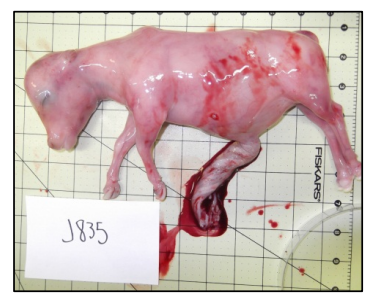

LOS \#4: 714g

Figure S1. Day $\sim 105$ fetuses used in this study. Each fetus is a B. t. indicus and B. t. taurus hybrid females. Fetus number and bodyweight are shown. Each square on the background = $2.54 \mathrm{~cm}^{2}$. Information on the fetus beyond that obtained in this study may be found in the publication (Chen et al., 2013).

A

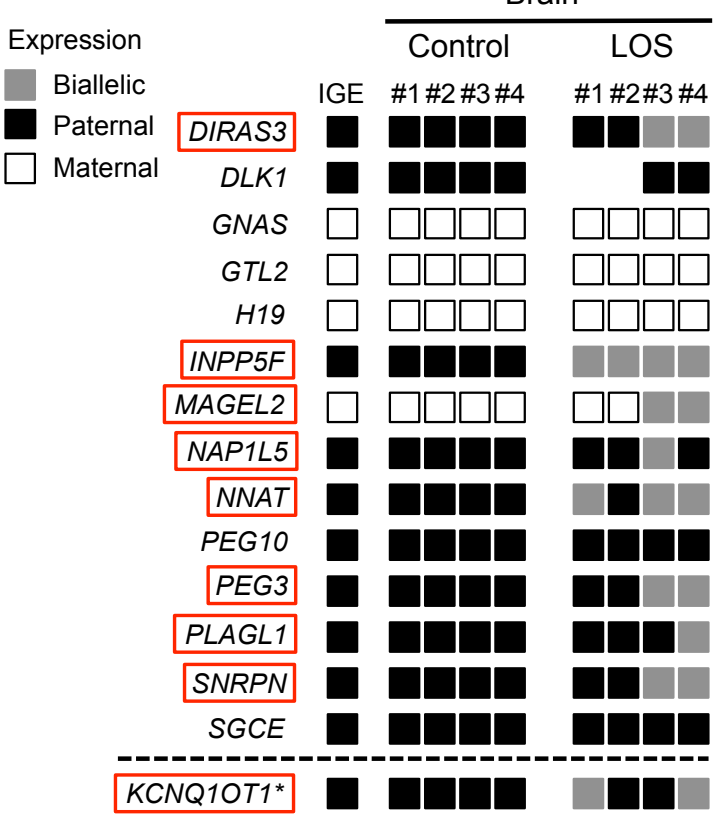

B Brain (\% of the repressed allele)

Control LOS

\begin{tabular}{|c|c|c|c|c|c|c|c|c|}
\hline & & 42 & \#3 & $\# 4$ & $\# 1$ & $\# 2$ & $\# 3$ & $\# 4$ \\
\hline DIRAS3 & 0 & . & 0 & 0 & 0 & 0 & 33.4 & 31.9 \\
\hline DLK1 & & & & & LE & LE & 0 & 0 \\
\hline GNAS & & & & & & & 0 & 0 \\
\hline$\angle 2$ & 0 & & & & & & & \\
\hline 119 & 0 & 0 & & 0 & 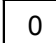 & 0 & 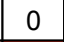 & 0 \\
\hline INPP5F & 0 & 0 & & 0 & 21 & 24.6 & 46.5 & 20. \\
\hline AGEL2 & 0 & & & 0 & 0 & 0 & $|17.7|$ & 26 \\
\hline NAP1L5 & 0 & 0 & 0 & 0 & 0 & 0 & 16.5 & $\infty$ \\
\hline NNAT & 0 & 0 & & 0 & 20.7 & 0 & 51.6 & 28 \\
\hline PEG10 & 0 & 0 & & 0 & 0 & & 0 & 0 \\
\hline PEG3 & 0 & 0 & 0 & 0 & & 0 & 18 & 2 \\
\hline PLAGL1 & 0 & 0 & 0 & 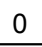 & 0 & 0 & 0 & 23 \\
\hline SNRPN & U & 0 & 0 & 0 & 0 & 0 & 18 & 24.5 \\
\hline & & & & 0 & 0 & & 0 & \\
\hline $10 T 1$ & 0 & 0 & 0 & $C$ & & & 0 & \\
\hline
\end{tabular}

Figure S2. Allele-specific expression analysis of known imprinted genes using RNAseq in brain of the fetuses. Details are as in Figure 1. LE=low expression (fewer than 10 reads). 
A

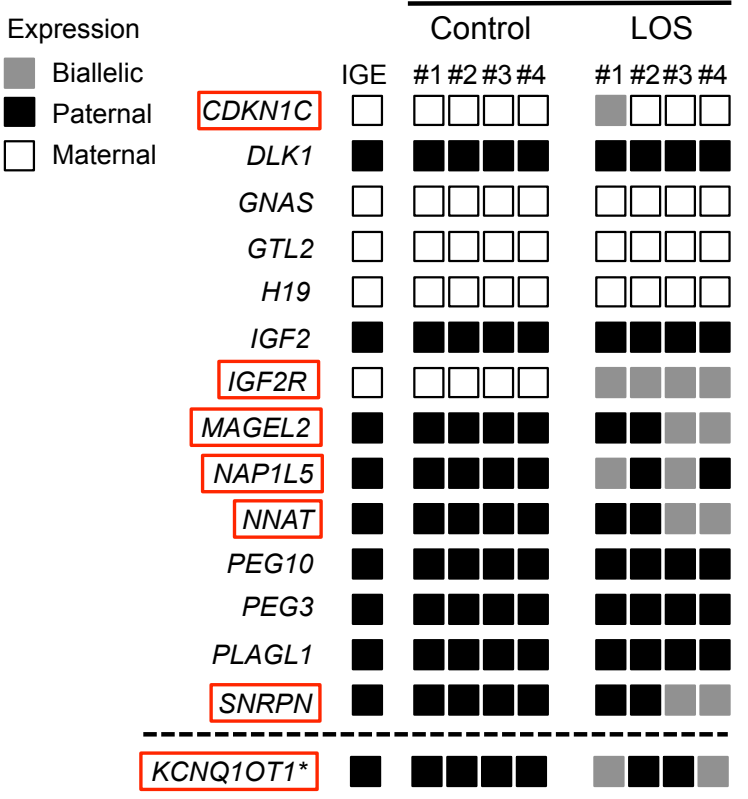

B Liver (\% of the repressed allele)

Control LOS

\begin{tabular}{|c|c|c|c|c|c|c|c|c|}
\hline & $\# 1$ & \#2 & \#3 & $\$ 4$ & $\# 1$ & $\# 2$ & $\# 3$ & $\# 4$ \\
\hline CDKN1C & 0 & 0 & 0 & 0 & 36.1 & 0 & 0 & 0 \\
\hline DLK1 & 0 & 0 & 0 & 0 & 0 & 0 & 0 & 0 \\
\hline GNAS & 0 & 0 & 0 & 0 & 0 & $\underline{0}$ & 0 & 0 \\
\hline GTL2 & 0 & 0 & 0 & 0 & 0 & 0 & 0 & 0 \\
\hline$H 19$ & 0 & 0 & 0 & 0 & 0 & 0 & 0 & 0 \\
\hline IGF2 & 0 & & & & 0 & & 0 & \\
\hline
\end{tabular}

\begin{tabular}{|l|l|l|l|l|l|l|l|l|l|l|}
\hline IGF2R & 0 & 0 & 0 & 0 & 42 & 23.2 & 27.4 & 21.7 \\
\hline
\end{tabular}

\begin{tabular}{|c|c|c|c|c|c|c|c|c|}
\hline MAGEL2 & 0 & 0 & 0 & 0 & 0 & 0 & 19.6 & 23.5 \\
\hline
\end{tabular}

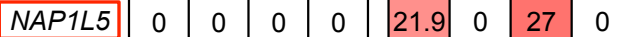

\begin{tabular}{|l|l|l|l|l|l|l|l|l|l|}
\hline NNAT & 0 & 0 & 0 & 0 & 0 & 0 & 34.8 & 19.5 \\
\hline
\end{tabular}

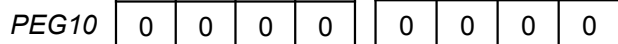

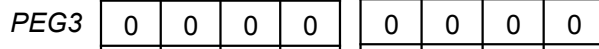

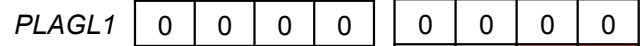

\begin{tabular}{|l|l|l|l|l|l|l|l|l|l|l|}
\hline SNRPN & 0 & 0 & 0 & 0 & 0 & 0 & 18.7 & 30.1 \\
\hline
\end{tabular}

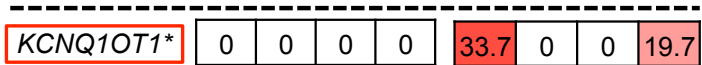

Figure S3. Allele-specific expression analysis of known imprinted genes using RNAseq in liver of the fetuses. Details are as in Figure 1. LE=low expression (fewer than 10 reads).

A

Expression

Biallelic

Paternal

Maternal

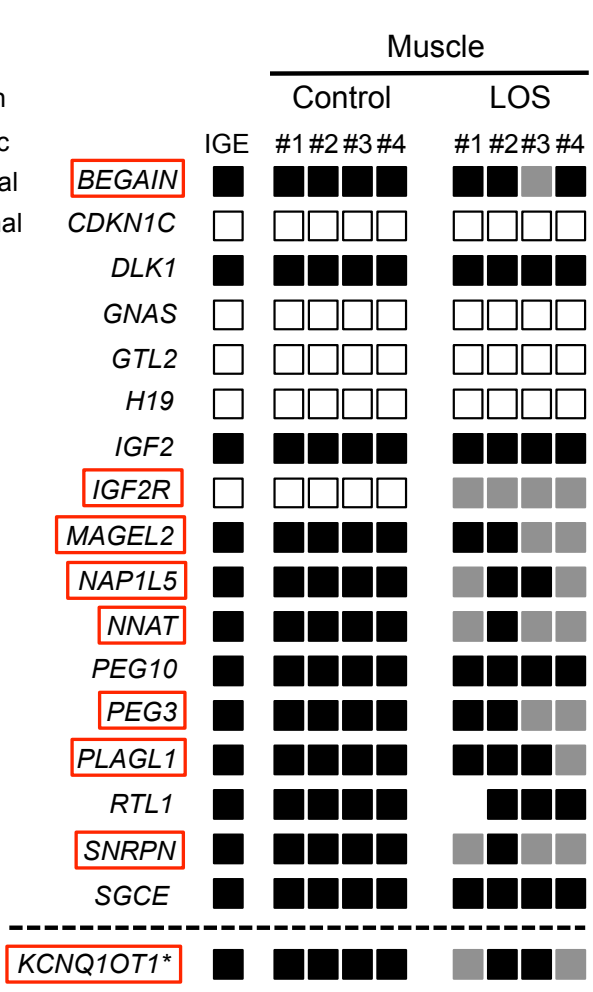

B

Muscle (\% of the repressed allele)

Control

LOS

BEGAIN

CDKN1C

$D L K 1$

GNAS

GTL2

$H 19$

IGF2

IGF2R

\begin{tabular}{|c|c|c|c|c|c|c|c|c|}
\hline MAGEL2 & 0 & 0 & 0 & 0 & 0 & 0 & 24.7 & 36.1 \\
\hline
\end{tabular}

\begin{tabular}{|l|l|l|l|l|}
\hline NAP1L5 & 0 & 0 & 0 & 0 \\
\hline
\end{tabular}

\begin{tabular}{|l|l|l|l|l|}
\hline NNAT & 0 & 0 & 0 & 0 \\
\hline
\end{tabular}

PEG10 000000

\begin{tabular}{|l|l|l|l|l|}
\hline PEG3 & 0 & 0 & 0 & 0 \\
\hline
\end{tabular}

\begin{tabular}{|l|l|l|l|l|}
\hline PLAGL1 & 0 & 0 & 0 & 0 \\
\hline
\end{tabular}

\begin{tabular}{l|l|l|l|l|}
$R T L 1$ & 0 & 0 & 0 & 0 \\
\cline { 2 - 5 }
\end{tabular}

\begin{tabular}{|l|l|l|l|l|}
\hline SNRPN & 0 & 0 & 0 & 0 \\
\hline
\end{tabular}

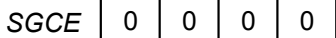

\begin{tabular}{|c|c|c|c|}
\hline$\# 1$ & \#2 & \#3 & $\# 4$ \\
\hline 0 & 0 & 24.5 & 0 \\
\hline 0 & 0 & 0 & 0 \\
\hline 0 & 0 & 0 & U \\
\hline 0 & 0 & 0 & U \\
\hline 0 & 0 & 0 & 0 \\
\hline 0 & 0 & 0 & 0 \\
\hline 0 & 0 & 0 & 0 \\
\hline 20.7 & 20.7 & 24 & 20.9 \\
\hline 0 & 0 & 24.7 & 36.1 \\
\hline 19.3 & 0 & 0 & 16.2 \\
\hline 31 & 0 & 45.7 & 45.7 \\
\hline 0 & 0 & 0 & 0 \\
\hline 0 & 0 & 20.5 & 39.2 \\
\hline 0 & 0 & 0 & 39.2 \\
\hline NA & 0 & 0 & 0 \\
\hline 18.6 & 0 & 21.3 & 41.6 \\
\hline 0 & 0 & 0 & 0 \\
\hline & 0 & 0 & \\
\hline
\end{tabular}

Figure S4. Allele-specific expression analysis of known imprinted genes using RNAseq in skeletal muscle of the fetuses. Details are as in Figure 1. NA: not assessable due to lack of subspecies-specific SNPs. 


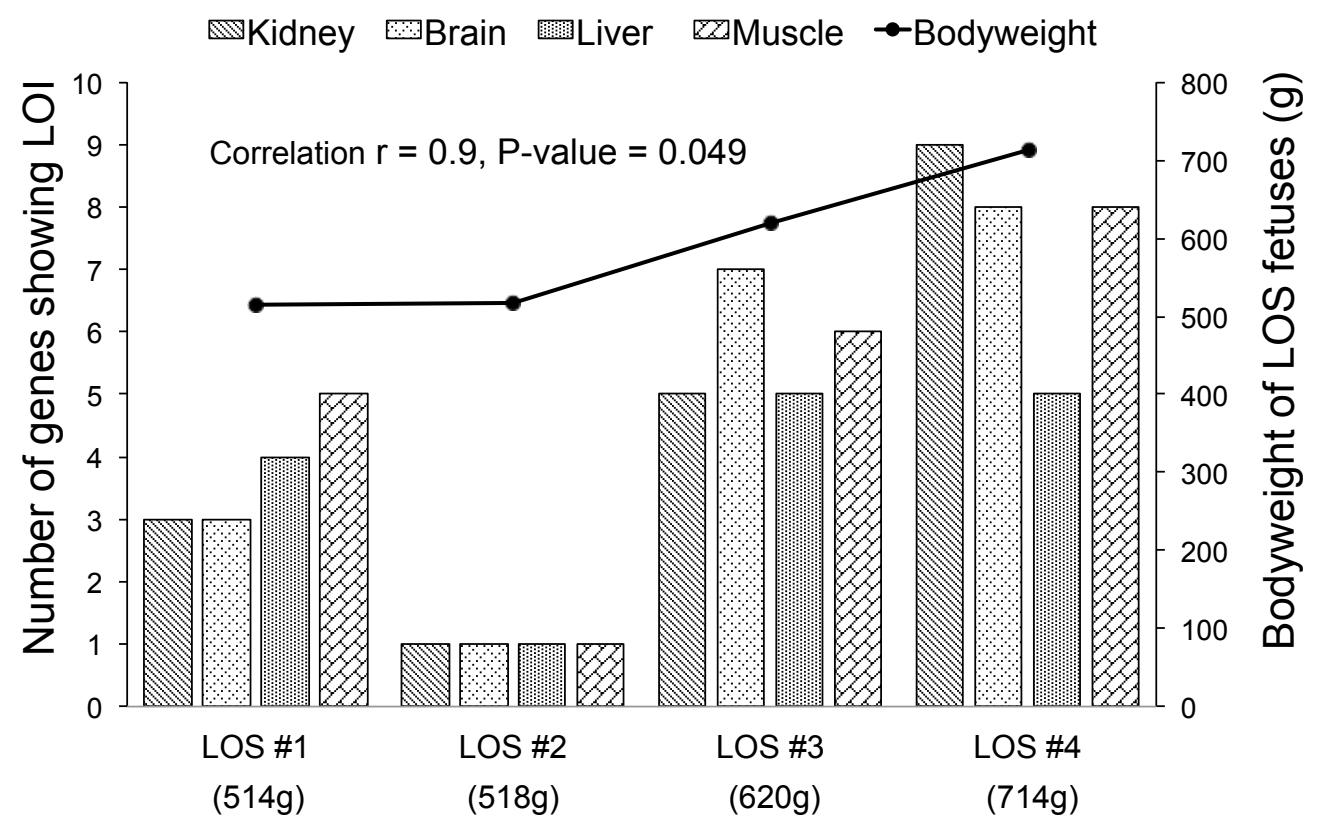

Figure S5. Positive correlation between number of genes showing LOI and bodyweight of LOS fetuses. Each bar represents the number of imprinted genes showing abnormal allelic expression in kidney, brain, liver and muscle of each LOS fetus. The bodyweight of each LOS fetus is illustrated by the connected black dots. The Pearson correlation coefficient between the number of genes showing LOI and the bodyweight of LOS fetuses is $0.90(\mathrm{P}=0.049)$. Allelic expression of KCNQ1OT1 was also included in the analysis.

PEG3

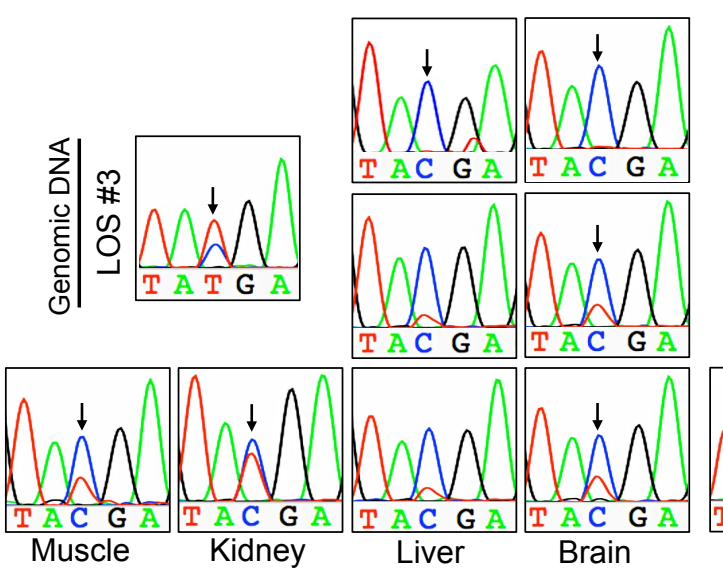

\section{SNRPN}

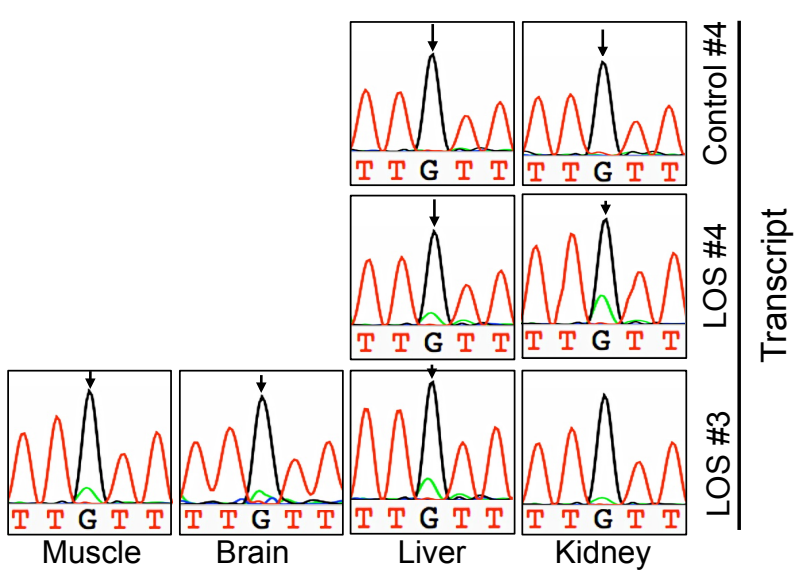

Figure S6. Verification of the allelic expression of PEG3, and $S N R P N$ in control and LOS fetuses. Shown is Sanger sequencing of RT-PCR amplicons of PEG3 and $S N R P N$. Double peaks pointed by arrows demonstrate biallelic expression. PEG3 in LOS \#3 and \#4 liver, and $S N R P N$ in LOS\#3 kidney were not considered biallelically expressed because the ratios expressed from the repressed allele were below 15\% according to RNAseq data. Other details are the same as described in Figure 2. 
Figure S7.
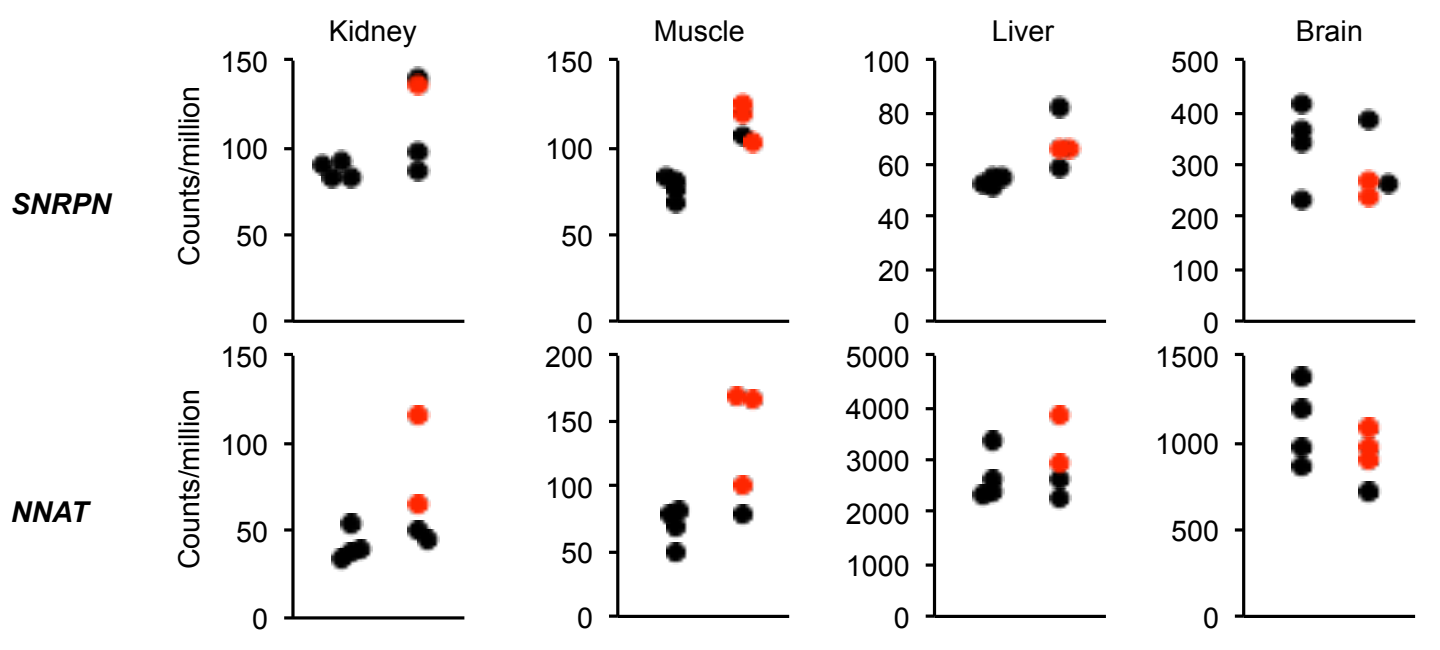

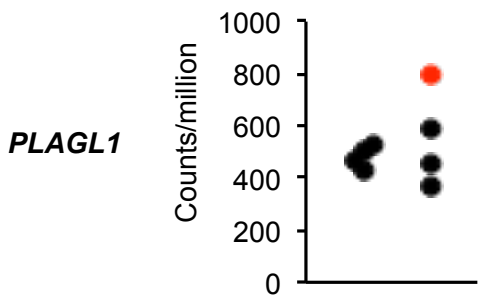
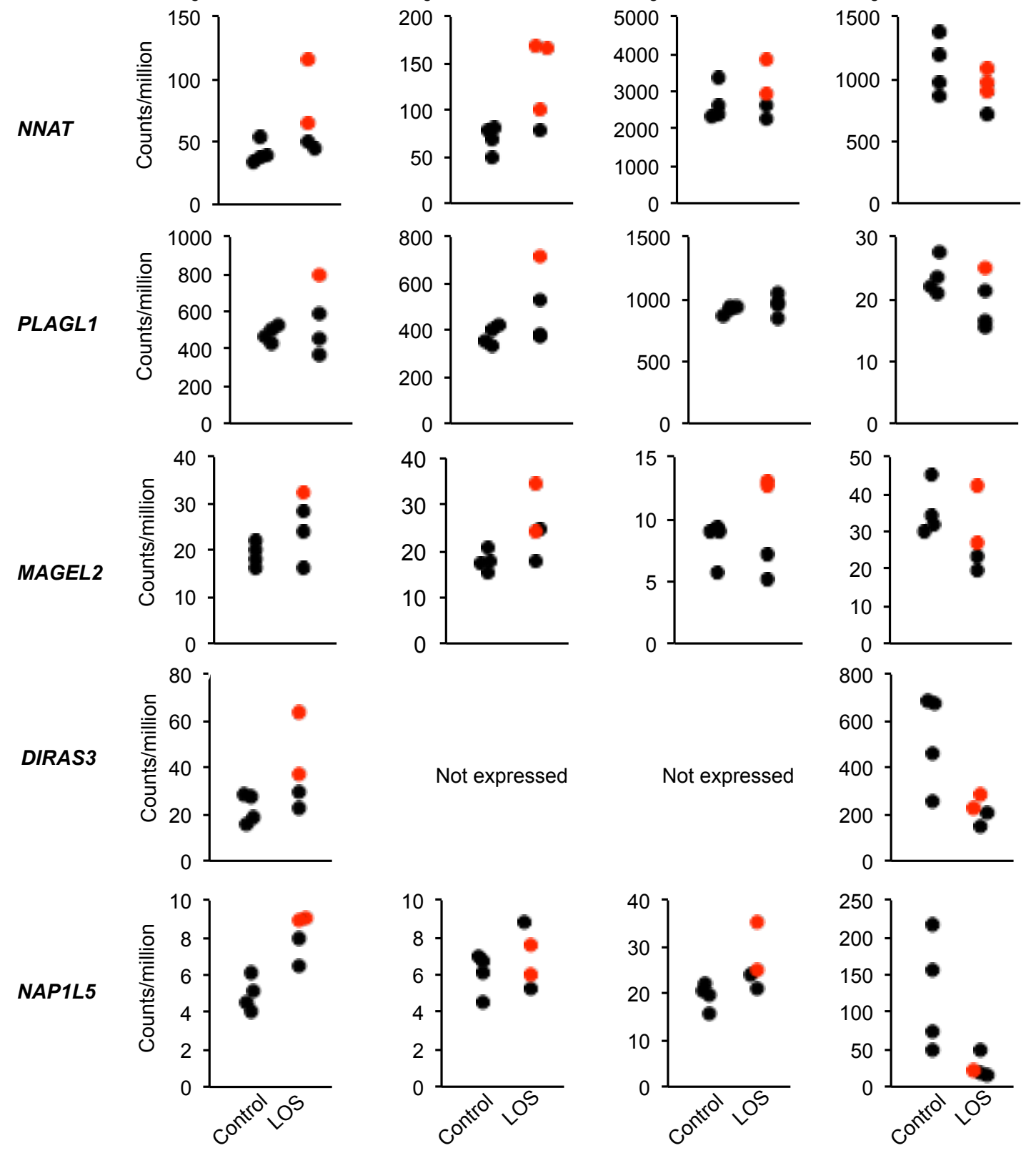


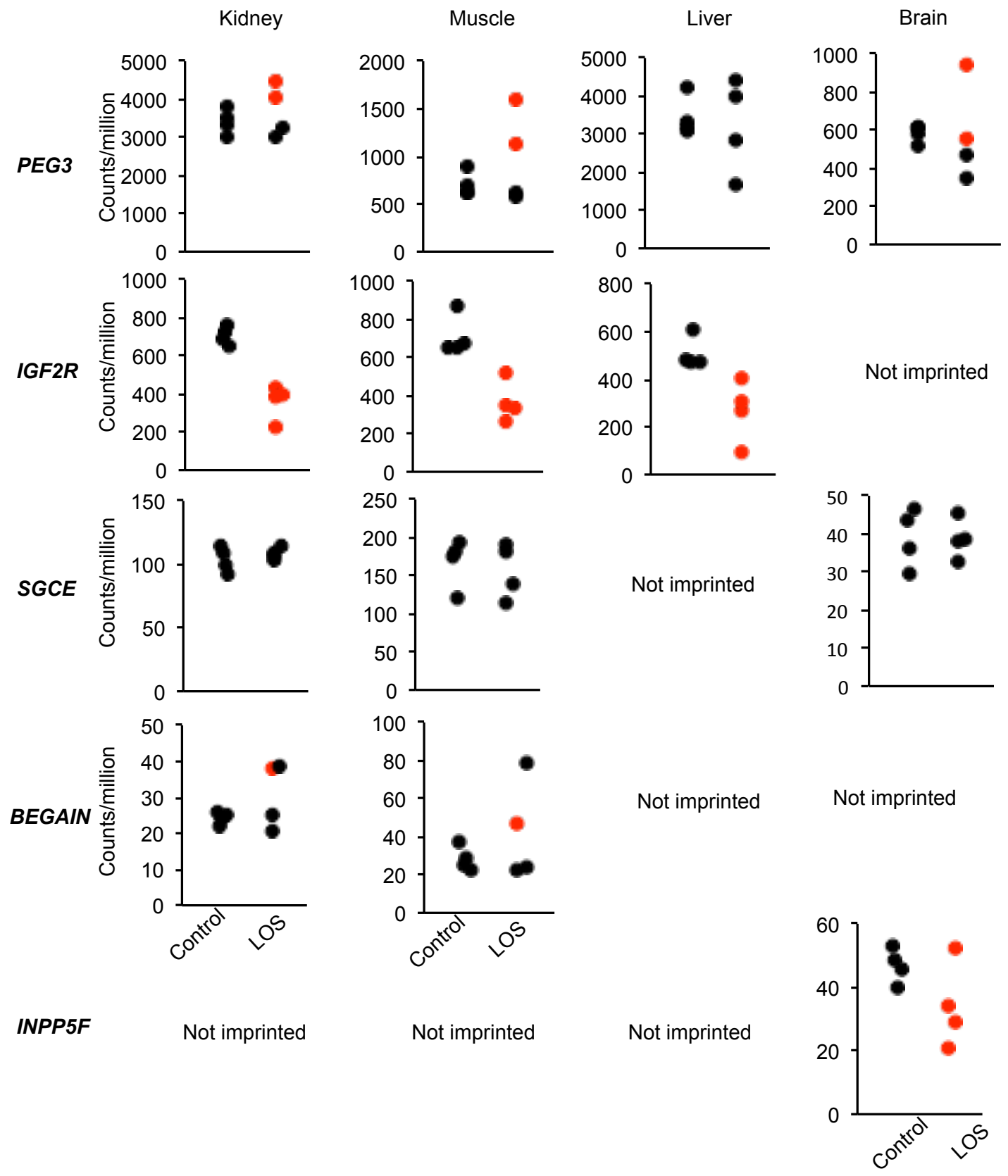

Figure S7. Lack of association between allelic expression and transcript abundance of imprinted genes. Transcript abundance was determined by the normalization of total RNAseq reads that aligned to the gene locus. Black dots represent genes with monoallelic expression and red dots represent genes with biallelic expression. 


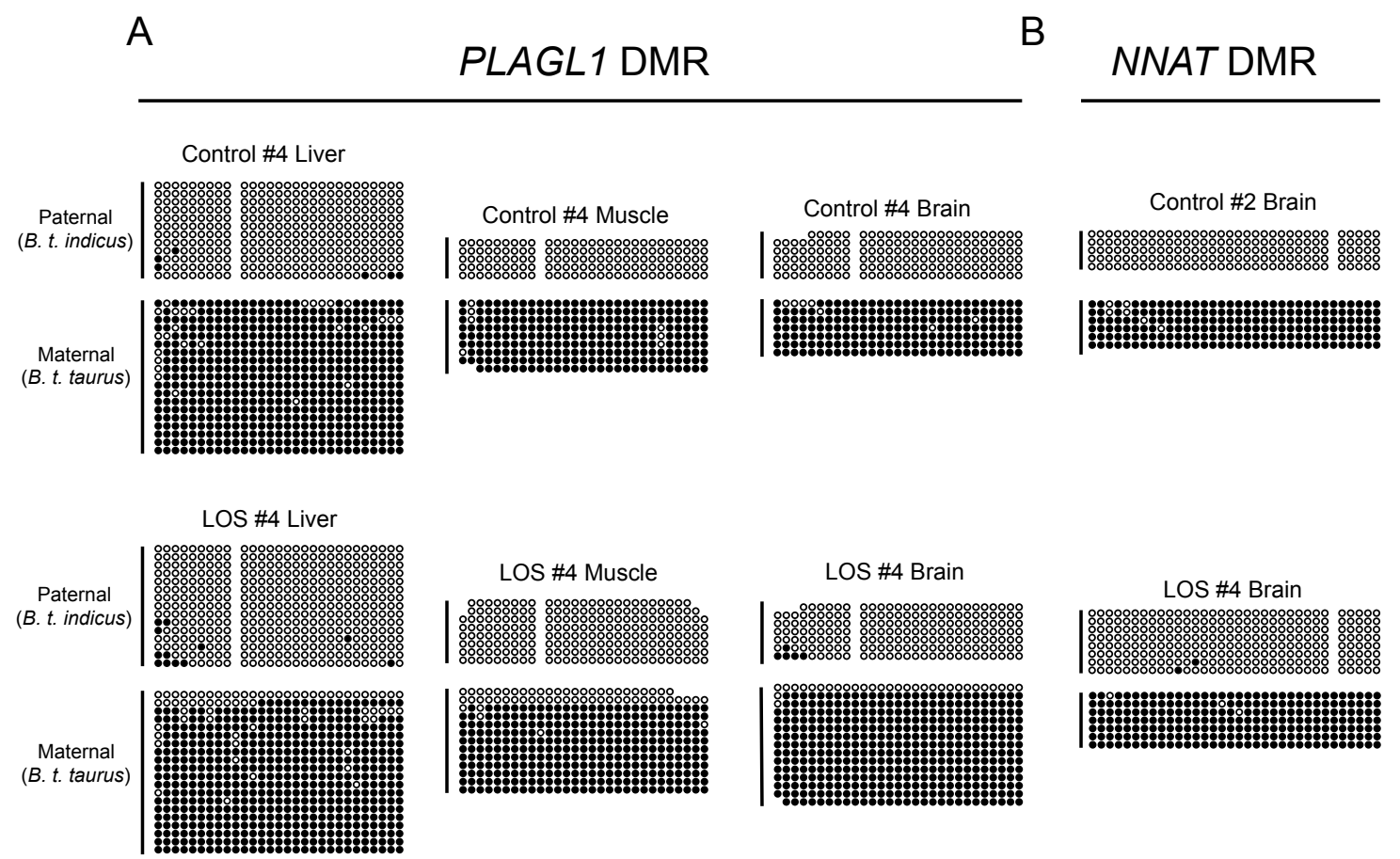

Figure S8. Allele-specific DNA methylation analysis of $P L A G L 1$ and $N N A T$ DMRs. (A). Methylation prolife of PLAGL1 DMR in liver, muscle, and brain of Control \#4 and LOS \#4 fetus. (B). Methylation profile of NNAT DMR in brain of Control \#2 and LOS \#4 LOS fetuses. Other details are the same as described in Figure 3. 

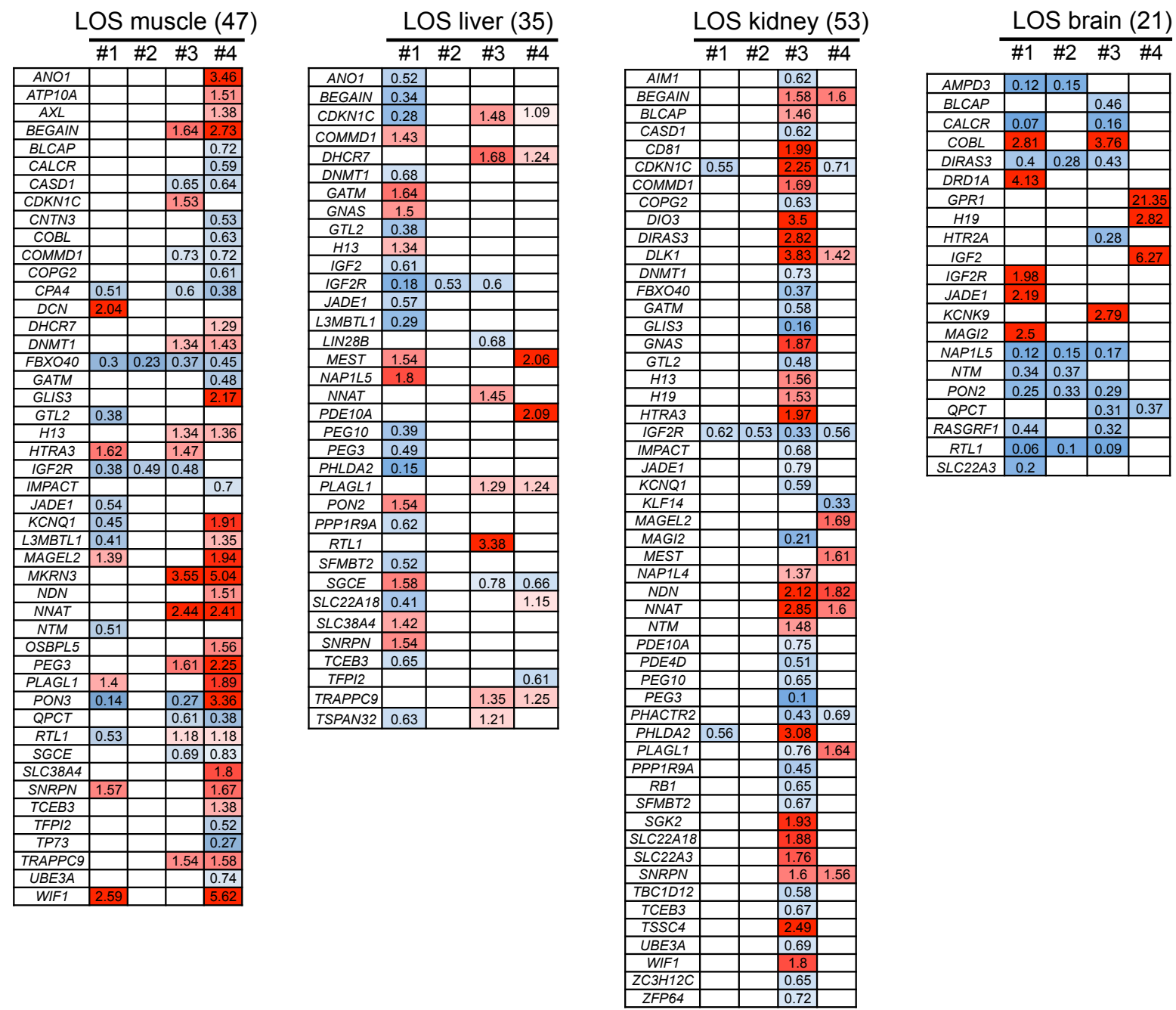

Figure S9. Differential expression of imprinted genes in LOS compared to controls. Heat map illustrating the differentially expressed imprinted genes identified using edgeR (false discovery rate $<0.05$ ) in each tissue of each LOS fetus (Blue = downregulated; Red = upregulated). Relative intensity of the color represents the level of fold change. Numbers in the table represent the transcript level relative to the average of controls. 
Table S1. B. t. indicus $\times$ B. t. taurus $F_{1}$ hybrid fetus information

\begin{tabular}{cccccccc}
\hline & & & & \multicolumn{4}{c}{ Organ Weight $(\mathrm{g})$} \\
\cline { 5 - 8 } Fetus & Sex & Fetal age & BW $(\mathrm{g})$ & Liver & Muscle & Brain & Kidney \\
\hline Control \#1 & Female & $\mathrm{d} 104$ & 392 & $13.4(3.4 \%)$ & ND & $12.8(3.3 \%)$ & $5.6(1.4 \%)$ \\
Control \#2 & Female & $\mathrm{d} 105$ & 404 & $14.9(3.7 \%)$ & ND & $11.3(2.8 \%)$ & $6.0(1.5 \%)$ \\
Control \#3 & Female & $\mathrm{d} 106$ & 408 & $13.9(3.4 \%)$ & ND & $12.0(2.9 \%)$ & $5.2(1.3 \%)$ \\
Control \#4 & Female & $\mathrm{d} 106$ & 416 & $14.1(3.4 \%)$ & ND & $11.7(2.8 \%)$ & $5.6(1.3 \%)$ \\
& & & & & & & \\
LOS \#1 & Female & $\mathrm{d} 106$ & $514^{*}$ & $23.3(4.5 \%)$ & ND & $11.6(2.3 \%)$ & $8.7(1.7 \%)$ \\
LOS \#2 & Female & $\mathrm{d} 105$ & $518^{*}$ & $19.4(3.7 \%)$ & ND & $11.6(2.2 \%)$ & $6.7(1.3 \%)$ \\
LOS \#3 & Female & $\mathrm{d} 106$ & $620^{*}$ & $27.7(4.5 \%)$ & ND & $11.7(1.9 \%)$ & $6.2(1.0 \%)$ \\
LOS \#4 & Female & $\mathrm{d} 105$ & $714^{*}$ & $26.8(3.8 \%)$ & ND & $11.2(1.6 \%)$ & $13.3(1.9 \%)$ \\
\hline
\end{tabular}

LOS: large offspring syndrome; BW: body weight (student t-test: LOS vs. Control * $\mathrm{P}=0.008$ ). Organ weights were also presented as the percentage of the bodyweight (as shown in parentheses), which were not statistically different between LOS and control fetuses. ND: not determined, only a piece of skeletal muscle was collected for analysis.

Table S2. Control and LOS fetal tissues RNAseq summary

\begin{tabular}{|c|c|c|c|c|c|c|c|c|}
\hline Fetus ID & Organ & Read length & $\begin{array}{c}\text { \# reads before quality } \\
\text { trimming }\end{array}$ & $\begin{array}{c}\text { \# reads after quality } \\
\text { trimming }\end{array}$ & $\begin{array}{c}\text { \# total reads mapped to } \\
\text { UMD3.1 }\end{array}$ & $\begin{array}{c}\% \text { total reads } \\
\text { mapped }\end{array}$ & $\begin{array}{c}\text { \# uniquely mapped } \\
\text { to UMD3.1 }\end{array}$ & $\begin{array}{l}\% \text { uniquely mapped } \\
\text { reads }\end{array}$ \\
\hline Control \#1 & Liver & $100 \mathrm{bp}$ & $49,324,460$ & $39,771,401$ & $37,440,644$ & 94.14 & $34,805,066$ & 87.51 \\
\hline Control \#1 & Muscle & $100 \mathrm{bp}$ & $35,012,854$ & $28,164,307$ & $25,873,674$ & 91.87 & $24,593,143$ & 87.32 \\
\hline Control \#1 & Brain & $100 \mathrm{bp}$ & $39,114,786$ & $31,602,386$ & $28,431,993$ & 89.97 & $27,434,118$ & 86.81 \\
\hline Control \#1 & Kidney & $100 \mathrm{bp}$ & $44,168,361$ & $35,748,305$ & $32,663,416$ & 91.37 & $31,188,516$ & 87.24 \\
\hline Control \#2 & Liver & $50 \mathrm{bp}$ & $89,453,260$ & $45,661,628$ & $43,683,972$ & 95.67 & $37,402,160$ & 81.91 \\
\hline Control \#2 & Muscle & $50 \mathrm{bp}$ & $56,538,563$ & $29,854,108$ & $28,126,835$ & 94.21 & $26,296,827$ & 88.08 \\
\hline Control \#2 & Brain & $50 \mathrm{bp}$ & $77,691,951$ & $38,651,699$ & $36,395,229$ & 94.16 & $33,913,437$ & 87.74 \\
\hline Control \#2 & Kidney & $100 \mathrm{bp}$ & $60,857,482$ & $43,334,190$ & $39,760,139$ & 91.75 & $37,999,230$ & 87.69 \\
\hline Control \#3 & Liver & $100 \mathrm{bp}$ & $47,032,906$ & $37,570,762$ & $35,616,256$ & 94.80 & $32,918,922$ & 87.62 \\
\hline Control \#3 & Muscle & $100 \mathrm{bp}$ & $36,876,793$ & $29,969,941$ & $27,970,927$ & 93.33 & $26,636,566$ & 88.88 \\
\hline Control \#3 & Brain & $100 \mathrm{bp}$ & $45,716,097$ & $37,136,173$ & $34,133,616$ & 91.91 & $32,718,553$ & 88.10 \\
\hline Control \#3 & Kidney & $100 \mathrm{bp}$ & $60,174,902$ & $41,681,288$ & $38,143,750$ & 91.51 & $36,302,479$ & 87.10 \\
\hline Control \#4 & Liver & $100 \mathrm{bp}$ & $48,359,824$ & $39,244,627$ & $36,949,505$ & 94.15 & $34,378,296$ & 87.60 \\
\hline Control \#4 & Muscle & $100 \mathrm{bp}$ & $32,704,016$ & $26,326,185$ & $23,929,859$ & 90.90 & $22,866,008$ & 86.86 \\
\hline Control \#4 & Brain & $100 \mathrm{bp}$ & $47,403,478$ & $38,145,390$ & $34,476,641$ & 90.38 & $33,229,226$ & 87.11 \\
\hline Control \#4 & Kidney & $100 \mathrm{bp}$ & $57,133,460$ & $40,562,200$ & $36,830,958$ & 90.80 & $35,088,062$ & 86.50 \\
\hline LOS \#1 & Liver & $100 \mathrm{bp}$ & $43,702,500$ & $35,039,540$ & $32,617,194$ & 93.09 & $29,689,819$ & 84.73 \\
\hline LOS \#1 & Muscle & $100 \mathrm{bp}$ & $36,677,201$ & $29,433,490$ & $26,969,294$ & 91.63 & $25,400,463$ & 86.30 \\
\hline LOS \#1 & Brain & $100 \mathrm{bp}$ & $34,368,056$ & $27,188,866$ & $25,718,282$ & 94.59 & $24,477,407$ & 90.03 \\
\hline LOS \#1 & Kidney & $100 \mathrm{bp}$ & $52,656,189$ & $39,385,040$ & $35,992,962$ & 91.39 & $34,353,786$ & 87.23 \\
\hline LOS \#2 & Liver & $100 \mathrm{bp}$ & $38,657,402$ & $31,204,376$ & $29,457,242$ & 94.40 & $27,117,333$ & 86.90 \\
\hline LOS \#2 & Muscle & $100 \mathrm{bp}$ & $46,224,766$ & $37,352,304$ & $34,653,583$ & 92.77 & $32,965,587$ & 88.26 \\
\hline LOS \#2 & Brain & $100 \mathrm{bp}$ & $43,052,038$ & $34,693,252$ & $32,524,533$ & 93.75 & $31,100,002$ & 89.64 \\
\hline LOS \#2 & Kidney & $100 \mathrm{bp}$ & $62,356,428$ & $43,043,803$ & $39,206,473$ & 91.09 & $37,295,278$ & 86.64 \\
\hline LOS \#3 & Liver & $100 \mathrm{bp}$ & $67,410,973$ & $49,344,697$ & $46,497,196$ & 94.23 & $43,052,525$ & 87.25 \\
\hline LOS \#3 & Muscle & $100 \mathrm{bp}$ & $66,164,453$ & $48,644,403$ & $45,352,608$ & 93.23 & $43,488,821$ & 89.40 \\
\hline LOS \#3 & Brain & $100 \mathrm{bp}$ & $76,645,582$ & $56,760,249$ & $52,959,888$ & 93.30 & $50,911,766$ & 89.70 \\
\hline LOS \#3 & Kidney & $100 \mathrm{bp}$ & $44,825,859$ & $32,386,152$ & $29,141,781$ & 89.98 & $27,305,679$ & 84.31 \\
\hline LOS \#4 & Liver & $100 \mathrm{bp}$ & $53,967,229$ & $43,237,304$ & $40,707,119$ & 94.15 & $37,594,353$ & 86.95 \\
\hline LOS \#4 & Muscle & $100 \mathrm{bp}$ & $56,742,110$ & $45,144,209$ & $41,889,305$ & 92.79 & $40,379,885$ & 89.45 \\
\hline LOS \#4 & Brain & $100 \mathrm{bp}$ & $79,150,645$ & $62,861,848$ & $58,386,058$ & 92.88 & $56,343,425$ & 89.63 \\
\hline LOS \#4 & Kidney & $100 \mathrm{bp}$ & $56,302,574$ & $38,654,962$ & $35,183,985$ & 91.02 & $33,495,182$ & 86.65 \\
\hline
\end{tabular}

LOS: large offspring syndrome; bp: base pairs 
Table S3. Allelic expression of the monoallelically expressed imprinted genes in $\sim \mathbf{d} 105$ bovine fetuses. For each tissue, left table shows the actual percentage of the expression from the repressed allele for each gene in each fetus. Right table shows the number of the reads covering the SNPs used for ascribing allelic expression of the imprinted gene in each fetus. Genes with biallelic expression in LOS but not in controls are delineated by a red box. LE = low expression (fewer than 10 reads); ND $=$ not detectable; $\mathrm{NA}=$ not assessable due to lack of subspecies-specific SNPs.

\begin{tabular}{|c|c|c|c|c|c|c|c|c|}
\hline & \multicolumn{8}{|c|}{ Brain ( $\%$ of the repressed allele) } \\
\hline & \multicolumn{4}{|c|}{ Control } & \multicolumn{4}{|c|}{ LOS } \\
\hline & \#1 & \#2 & \#3 & \#4 & \#1 & \#2 & \#3 & \#4 \\
\hline DIRAS3 & 0.1 & 0.0 & 0.3 & 0.2 & 6.1 & 6.3 & 33.4 & 31.9 \\
\hline DLK1 & 0.0 & 0.0 & 0.0 & 0.0 & LE & LE & 0.0 & 0.0 \\
\hline GNAS & 0.5 & 1.3 & 0.4 & 0.5 & 0.1 & 0.4 & 1.5 & 1.6 \\
\hline GTL2 & 0.0 & 0.2 & 0.1 & 0.1 & 0.0 & 0.1 & 0.0 & 0.5 \\
\hline$H 19$ & 12.1 & 6.7 & 14.2 & 4.0 & 0.0 & LE & 6.9 & 9.2 \\
\hline MAGEL2 & 0.0 & 0.0 & 0.0 & 0.6 & 6.7 & 3.2 & 17.7 & 26.5 \\
\hline NAP1L5 & 0.0 & 2.3 & 0.1 & 0.4 & 9.8 & 9.3 & 16.5 & 9.5 \\
\hline NNAT & 0.9 & 0.1 & 0.6 & 0.4 & 20.7 & 1.5 & 51.6 & 28.4 \\
\hline PEG10 & 0.6 & 0.0 & 0.2 & 0.1 & 0.1 & 0.1 & \begin{tabular}{|l|}
0.0 \\
\end{tabular} & 0.1 \\
\hline PEG3 & 0.0 & 0.6 & 0.4 & 0.0 & 0.0 & 7.8 & 18.0 & 28.0 \\
\hline PLAGL1 & 1.2 & 1.8 & 1.1 & 2.7 & 2.5 & 1.5 & 0.8 & 23.1 \\
\hline RTL1 & ND & ND & ND & ND & ND & ND & ND & ND \\
\hline SNRPN & 0.0 & 0.0 & 0.0 & 0.0 & 6.2 & 5.3 & 18.0 & 24.5 \\
\hline BEGAIN & 23.8 & 46.7 & 38.8 & 32.9 & 58.6 & 14.6 & 43.1 & 40.3 \\
\hline$C D K N 1 C$ & 11.4 & 11.1 & 20.0 & 13.1 & 18.2 & 18.7 & 34.8 & 32.0 \\
\hline IGF2 & 38.9 & 19.0 & LE & 58.3 & 0.0 & 23.3 & 34.4 & 25.0 \\
\hline IGF2R & 51.1 & 41.6 & 45.8 & 59.4 & 54.2 & 49.7 & 47.5 & 41.5 \\
\hline INPP5F & 3.4 & 11.2 & 2.7 & 1.6 & 21.0 & 24.6 & 46.5 & 20.3 \\
\hline PHLDA2 & 50.0 & LE & LE & LE & LE & LE & LE & 70.0 \\
\hline$S G C E$ & 1.0 & 2.6 & 0.0 & 1.2 & 0.0 & 1.0 & 1.2 & 2.0 \\
\hline
\end{tabular}

Kidney (\% of the repressed allele)

\begin{tabular}{|c|c|c|c|c|c|c|c|c|}
\hline & \multicolumn{4}{|c|}{ Control } & \multicolumn{4}{|c|}{ LOS } \\
\hline & $\# 1$ & \#2 & \#3 & \#4 & \#1 & \#2 & \#3 & \#4 \\
\hline \begin{tabular}{|l|} 
DIRAS3 \\
\end{tabular} & \begin{tabular}{|l|}
1.5 \\
\end{tabular} & 0.0 & 0.5 & \begin{tabular}{|l|} 
\\
\end{tabular} & 3.0 & 3.5 & 51.3 & 41.2 \\
\hline DLK1 & \begin{tabular}{|l|}
0.2 \\
\end{tabular} & 0.0 & 0.3 & \begin{tabular}{|l|}
0.1 \\
\end{tabular} & 0.2 & 0.1 & 0.1 & 0.1 \\
\hline GNAS & 1.1 & 0.8 & 1.3 & 0.9 & 1.7 & 1.2 & 1.0 & 1.2 \\
\hline GTL2 & 1.0 & 0.7 & 0.5 & 0.5 & 1.1 & 0.6 & 1.6 & 1.2 \\
\hline$H 19$ & 3.3 & 3.3 & 3.5 & 3.6 & 3.8 & 3.8 & 3.8 & 3.8 \\
\hline MAGEL2 & \begin{tabular}{|l|}
0.0 \\
\end{tabular} & \begin{tabular}{|l|} 
\\
0.0
\end{tabular} & 0.0 & \begin{tabular}{|l|}
0.0 \\
\end{tabular} & 10.2 & 3.9 & 8.7 & 35.6 \\
\hline NAP1L5 & 0.0 & 13.0 & 0.0 & 2.4 & 25.8 & 6.5 & 6.5 & 15.5 \\
\hline NNAT & 0.9 & 5.3 & 2.2 & 2.5 & 7.3 & 0.6 & 43.1 & 41.8 \\
\hline PEG10 & \begin{tabular}{|l|}
0.1 \\
\end{tabular} & \begin{tabular}{|l|}
0.2 \\
\end{tabular} & 0.1 & \begin{tabular}{|l|}
0.1 \\
\end{tabular} & \begin{tabular}{|l|}
0.1 \\
\end{tabular} & 0.1 & \begin{tabular}{|l|}
0.4 \\
\end{tabular} & 0.1 \\
\hline PEG3 & 0.1 & 0.4 & 0.3 & 0.4 & 0.6 & 13.6 & 52.4 & 47.6 \\
\hline PLAGL1 & 0.6 & 0.4 & 0.6 & 1.3 & 0.3 & 0.7 & 0.5 & 35.5 \\
\hline RTL1 & 2.1 & 0.0 & 4.5 & 7.5 & NA & 2.2 & 12.0 & 3.4 \\
\hline SNRPN & 0.0 & 0.0 & 0.0 & 0.0 & 5.1 & 9.1 & 11.8 & 33.9 \\
\hline BEGAIN & 0.0 & 7.4 & 3.0 & 5.9 & 3.6 & 0.0 & 21.3 & 1.8 \\
\hline CDKN1C & 3.1 & 3.9 & 1.7 & 4.3 & 6.3 & 4.1 & 3.5 & 8.5 \\
\hline IGF2 & 0.2 & 0.1 & 0.2 & 0.1 & 0.4 & 0.1 & 0.1 & 0.1 \\
\hline IGF2R & 9.0 & 7.7 & 9.5 & 11.3 & 17.7 & 15.1 & 32.2 & 21.7 \\
\hline INPP5F & 36.5 & 36.5 & 2.0 & 3.4 & 49.7 & 38.6 & 50.6 & 13.4 \\
\hline PHLDA2 & 17.6 & 18.6 & 11.2 & 9.7 & 15.7 & 8.7 & 5.8 & 12.7 \\
\hline SGCE & 0.2 & 0.2 & 0.0 & 0.0 & 0.6 & 0.0 & 2.1 & 0.9 \\
\hline
\end{tabular}

Brain ( \# of reads containing the SNPs used to ascribe the allelic expression)

\begin{tabular}{|c|c|c|c|c|c|c|c|c|c|}
\hline & \multicolumn{8}{|c|}{$\begin{array}{l}\text { Brain (\# of reads containing the SNPs } \\
\text { used to ascribe the allelic expression) }\end{array}$} & \\
\hline & \multicolumn{4}{|c|}{ Control } & \multicolumn{4}{|c|}{ LOS } & \\
\hline & $\# 1$ & \#2 & \#3 & $\# 4$ & $\# 1$ & $\# 2$ & \#3 & $\# 4$ & \\
\hline \begin{tabular}{|l|} 
DIRAS3 \\
\end{tabular} & 3627 & 696 & 2777 & 4629 & 783 & 821 & 2022 & 2993 & \\
\hline DLK1 & 28 & 18 & 59 & 62 & 3 & 7 & 11 & 191 & \\
\hline GNAS & 1932 & 1012 & 2071 & 2052 & 1425 & 1390 & 1579 & 2326 & \\
\hline GTL2 & 877 & 929 & 885 & 719 & 508 & 735 & 1180 & 1959 & \\
\hline$H 19$ & 41 & 30 & 21 & 25 & 9 & 20 & 160 & 577 & \\
\hline MAGEL2 & 109 & 50 & 99 & 165 & 60 & 62 & 96 & 177 & \\
\hline \begin{tabular}{|l|} 
NAP1L5 \\
\end{tabular} & 2004 & 308 & 805 & 1715 & 102 & 140 & 309 & 979 & \\
\hline \begin{tabular}{|l|} 
NNAT \\
\end{tabular} & 3390 & 1037 & 3804 & 11825 & 733 & 4883 & 2355 & 13430 & \\
\hline PEG10 & 5225 & 3585 & 6097 & 7762 & 6327 & 5430 & 7472 & 11290 & \\
\hline PEG3 & 262 & 154 & 230 & 225 & 137 & 154 & 900 & 1770 & \\
\hline \begin{tabular}{|l|} 
PLAGL1 \\
\end{tabular} & 81 & 57 & 95 & 73 & 40 & 67 & 124 & 221 & \\
\hline RTL1 & 0 & 0 & 0 & 0 & 0 & 0 & 0 & 0 & \\
\hline SNRPN & 1971 & 498 & 1758 & 2477 & 1823 & 1472 & 1760 & 2311 & \\
\hline$B E G A I N$ & 63 & 30 & 103 & 82 & 29 & 48 & 58 & 139 & \\
\hline CDKN1C & 44 & 18 & 50 & 76 & 11 & 16 & 66 & 72 & \\
\hline IGF2 & 72 & 79 & 7 & 36 & 17 & 43 & 122 & 776 & \\
\hline IGF2R & 223 & 173 & 194 & 180 & 306 & 316 & 547 & 695 & \\
\hline INPP5F & 5133 & 1013 & 589 & 733 & 2123 & 3055 & 6452 & 1132 & \\
\hline PHLDA2 & 14 & 3 & 6 & 6 & 3 & 4 & 6 & 10 & \\
\hline \multirow[t]{4}{*}{ SGCE } & 186 & 77 & 265 & 254 & 119 & 198 & 257 & 350 & \\
\hline & \multicolumn{8}{|c|}{$\begin{array}{l}\text { Kidney (\# of reads containing the SNPs } \\
\text { used to ascribe the allelic expression) }\end{array}$} & \\
\hline & \multicolumn{4}{|c|}{ Control } & \multicolumn{4}{|c|}{ LOS } & \\
\hline & $\# 1$ & \#2 & \#3 & $\# 4$ & \#1 & $\# 2$ & \#3 & \#4 & \\
\hline \begin{tabular}{|l|} 
DIRAS3 \\
\end{tabular} & 132 & 202 & 211 & 105 & 199 & 144 & 386 & 262 & \\
\hline$D L K 1$ & 2391 & 2564 & 2672 & 2817 & 3352 & 3324 & 8785 & 3686 & \\
\hline GNAS & 1184 & 1470 & 1351 & 1322 & 1295 & 1691 & 1240 & 1836 & \\
\hline GTL2 & 706 & 839 & 547 & 650 & 797 & 637 & 124 & 650 & \\
\hline H19 & 10705 & 13994 & 12275 & 16069 & 14507 & 13336 & 15148 & 15991 & \\
\hline MAGEL2 & 53 & 67 & 65 & 38 & 59 & 76 & 57 & 101 & \\
\hline NAP1L5 & 52 & 46 & 46 & 42 & 93 & 61 & 62 & 71 & \\
\hline NNAT & 3390 & 19 & 185 & 276 & 41 & 326 & 65 & 491 & \\
\hline PEG10 & 4571 & 5922 & 4568 & 4776 & 5395 & 4591 & 972 & 4311 & \\
\hline \begin{tabular}{|l|}
$P E G 3$ \\
\end{tabular} & 1755 & 1855 & 2048 & 1091 & \begin{tabular}{|l|}
1316 \\
\end{tabular} & 1541 & 248 & 2195 & \\
\hline PLAGL1 & 1257 & 1547 & 1396 & 1076 & 1263 & 1225 & 425 & 2966 & \\
\hline RTL1 & 48 & 18 & 44 & 40 & NA & 46 & 67 & 89 & \\
\hline SNRPN & 348 & 433 & 379 & 355 & 375 & 482 & 382 & 613 & \\
\hline BEGAIN & 22 & 27 & 33 & 34 & 28 & 24 & 47 & 55 & \\
\hline CDKN1C & 292 & 281 & 290 & 276 & 159 & 318 & 517 & 200 & \\
\hline IGF2 & 9227 & 11520 & 11107 & 11709 & \begin{tabular}{|l|l}
9578 \\
\end{tabular} & 10549 & 8825 & 10075 & \\
\hline IGF2R & 2674 & 3459 & 3002 & 2706 & 1754 & 1661 & 556 & 1601 & \\
\hline INPP5F & 356 & 389 & 50 & 29 & 541 & 485 & 259 & 67 & \\
\hline PHLDA2 & 262 & 306 & 259 & 421 & 210 & 392 & 1003 & 291 & \\
\hline SGCE & 556 & 510 & 569 & 465 & 514 & 663 & 517 & 633 & \\
\hline
\end{tabular}


Table S3. Allelic expression of the monoallelically expressed imprinted genes in $\sim$ d105 bovine fetuses.

\begin{tabular}{|c|c|c|c|c|c|c|c|c|}
\hline & \multicolumn{8}{|c|}{ Liver (\% of the repressed allele) } \\
\hline & \multicolumn{4}{|c|}{ Control } & \multicolumn{4}{|c|}{ LOS } \\
\hline & \#1 & \#2 & \#3 & \#4 & \#1 & \#2 & \#3 & \#4 \\
\hline DIRAS3 & ND & ND & ND & ND & ND & ND & ND & ND \\
\hline DLK1 & 0.2 & 1.4 & 0.5 & 0.5 & 0.4 & 0.3 & 0.0 & 0.1 \\
\hline GNAS & 1.1 & 3.7 & 1.0 & 0.6 & 2.1 & 0.7 & 0.7 & 1.7 \\
\hline GTL2 & 0.0 & 0.6 & 0.5 & 0.5 & \begin{tabular}{|l|} 
\\
\end{tabular} & 0.2 & 0.3 & 1.1 \\
\hline$H 19$ & 3.6 & 1.8 & 5.8 & 6.2 & \begin{tabular}{|l|}
5.5 \\
\end{tabular} & 7.1 & 5.8 & \begin{tabular}{|l|}
5.4 \\
\end{tabular} \\
\hline MAGEL2 & 0.0 & 0.0 & 0.0 & 0.0 & 3.8 & 3.8 & 19.6 & 23.5 \\
\hline NAP1L5 & 0.0 & 4.3 & 0.0 & 0.0 & 21.9 & 5.4 & 27.0 & 3.0 \\
\hline NNAT & 0.1 & 0.1 & 0.9 & 0.3 & 1.0 & 0.4 & 34.8 & 19.5 \\
\hline PEG10 & 0.0 & 0.0 & 0.1 & 0.1 & 0.2 & 0.3 & 0.1 & 0.1 \\
\hline PEG3 & 0.1 & 0.0 & 0.1 & 0.1 & 1.1 & 0.1 & 14.1 & 12.5 \\
\hline PLAGL1 & 0.1 & 0.1 & 0.1 & 0.1 & \begin{tabular}{|l|}
0.1 \\
\end{tabular} & 0.1 & 3.7 & \begin{tabular}{|l|}
8.6 \\
\end{tabular} \\
\hline$R T L 1$ & ND & ND & ND & ND & ND & ND & ND & ND \\
\hline SNRPN & 0.0 & 0.0 & 0.0 & 0.0 & 7.9 & 4.5 & 18.7 & 30.1 \\
\hline BEGAIN & 13.3 & 0.0 & 21.1 & 13.3 & LE & 0.0 & \begin{tabular}{|l|} 
\\
\end{tabular} & 0.0 \\
\hline CDKN1C & 5.0 & 5.0 & 7.3 & 9.9 & 36.1 & 7.5 & 9.4 & 6.0 \\
\hline IGF2 & 0.1 & 0.0 & 0.0 & 0.0 & 0.0 & 0.0 & 0.0 & \begin{tabular}{|l|} 
\\
\end{tabular} \\
\hline IGF2R & 14.2 & 13.6 & 14.8 & 14.0 & 42.0 & 23.2 & 27.4 & 21.7 \\
\hline INPP5F & 32.4 & 37.4 & 2.0 & 3.4 & 50.3 & 35.5 & 44.1 & 19.0 \\
\hline PHLDA2 & 5.0 & LE & 0.0 & 0.0 & LE & $\mathrm{LE}$ & LE & 21.1 \\
\hline SGCE & 25.2 & 33.1 & 24.0 & 30.1 & 36.2 & 24.1 & 26.8 & 16.3 \\
\hline
\end{tabular}

\begin{tabular}{|c|c|c|c|c|c|c|c|c|}
\hline & \multicolumn{8}{|c|}{ Muscle (\% of the repressed allele) } \\
\hline & \multicolumn{4}{|c|}{ Control } & \multicolumn{4}{|c|}{ LOS } \\
\hline & $\# 1$ & $\# 2$ & \#3 & $\# 4$ & $\# 1$ & $\# 2$ & \#3 & $\# 4$ \\
\hline DIRAS3 & ND & ND & ND & ND & ND & ND & ND & ND \\
\hline DLK1 & 0.4 & 1.0 & 0.3 & 0.2 & 0.5 & 0.4 & 0.1 & 0.1 \\
\hline GNAS & 0.4 & 2.8 & 0.4 & 0.6 & 1.0 & 0.2 & 1.4 & 1.6 \\
\hline GTL2 & 0.2 & 0.2 & 0.5 & 0.3 & 0.5 & 0.2 & 0.4 & 0.3 \\
\hline H19 & 3.8 & 4.5 & 4.6 & 5.7 & 4.7 & 4.4 & 4.4 & 5.3 \\
\hline MAGEL2 & 0.0 & 0.0 & 0.0 & 4.2 & 10.3 & 10.9 & 24.7 & 36.1 \\
\hline NAP1L5 & 0.0 & 2.5 & 0.0 & 0.0 & 19.3 & 10.0 & $\mid 4.1$ & 16.2 \\
\hline NNAT & 6.3 & 3.4 & 4.0 & 13.5 & 31.0 & 2.6 & 45.7 & 45.7 \\
\hline PEG10 & 0.2 & 0.1 & 0.2 & 0.2 & 0.2 & 0.3 & 0.1 & 0.8 \\
\hline PEG3 & 0.6 & 0.0 & 0.3 & 0.0 & 0.0 & 7.2 & 20.5 & 39.2 \\
\hline \begin{tabular}{|l|} 
PLAGL1 \\
\end{tabular} & 1.9 & 1.1 & 0.6 & 1.9 & 0.5 & 1.2 & 0.4 & 39.2 \\
\hline RTL1 & 2.1 & 2.7 & 3.1 & 7.0 & NA & 2.8 & 5.4 & 1.4 \\
\hline SNRPN & 0.0 & 0.0 & 0.0 & 0.0 & 18.6 & 11.4 & 21.3 & 41.6 \\
\hline BEGAIN & 0.0 & 0.0 & 0.0 & 5.3 & 3.3 & 3.2 & 24.5 & 10.8 \\
\hline CDKN1C & 5.9 & 4.2 & 6.0 & 5.3 & 4.7 & 2.7 & 4.2 & 11.7 \\
\hline IGF2 & 0.5 & 0.5 & 0.6 & 0.5 & 0.4 & 0.2 & 0.4 & 1.0 \\
\hline IGF2R & 8.9 & 7.7 & 11.1 & 8.6 & 20.7 & 20.7 & 24.0 & 20.9 \\
\hline INPP5F & 47.2 & 52.5 & 1.1 & 1.4 & 51.9 & 46.3 & 53.5 & 19.0 \\
\hline PHLDA2 & LE & LE & LE & ND & LE & LE & ND & LE \\
\hline$S G C E$ & 1.6 & 2.2 & 1.8 & 0.9 & 2.2 & 0.8 & 2.4 & 10.3 \\
\hline
\end{tabular}

\begin{tabular}{|c|c|c|c|c|c|c|c|c|}
\hline & & $\begin{array}{l}\text { Liv } \\
\text { us }\end{array}$ & ( \# & rea & & & $\epsilon$ & \\
\hline & & Cont & & & & & OS & \\
\hline & $\# 1$ & $\# 2$ & $\# 3$ & $\# 4$ & $\# 1$ & \#2 & $\# 3$ & $\# 4$ \\
\hline DIRAS3 & 0 & 0 & 0 & 0 & 0 & 0 & 0 & 0 \\
\hline DLK1 & 4330 & 1898 & 4290 & 3919 & 3883 & 2570 & 4452 & 3875 \\
\hline GNAS & 807 & 815 & 894 & 837 & 807 & 568 & 1069 & 922 \\
\hline GTL2 & 628 & 787 & 424 & 650 & 185 & 432 & 953 & 1008 \\
\hline$H 19$ & 7771 & 4854 & 7997 & \begin{tabular}{|l|}
8778 \\
\end{tabular} & 6513 & 4520 & 22867 & 24216 \\
\hline MAGEL2 & 44 & 23 & 11 & 43 & 26 & 26 & 56 & 34 \\
\hline NAP1L5 & 240 & 116 & 251 & 175 & 333 & 204 & 392 & 305 \\
\hline NNAT & 12020 & 4039 & 9643 & 20024 & 2822 & 14834 & 7381 & 30639 \\
\hline PEG10 & 15788 & 10197 & 15286 & 16663 & 7629 & 12360 & 17495 & 12801 \\
\hline PEG3 & 1366 & 1232 & 1648 & 1430 & 179 & 1125 & 5778 & 4650 \\
\hline PLAGL1 & 3864 & 3815 & 2727 & 2890 & 1660 & 2896 & 5092 & 6560 \\
\hline RTL1 & 0 & 0 & 0 & 0 & 0 & 0 & 0 & 0 \\
\hline SNRPN & 296 & 125 & 272 & 294 & 302 & 262 & 417 & 418 \\
\hline BEGAIN & 15 & 12 & 19 & 15 & 0 & 10 & 23 & 14 \\
\hline CDKN1C & 219 & 100 & 246 & 242 & 61 & 133 & 394 & 283 \\
\hline IGF2 & 6849 & 6880 & 7332 & 7043 & 3167 & 4655 & 12894 & 11801 \\
\hline \begin{tabular}{|l|}
$I G F 2 R$ \\
\end{tabular} & 2461 & 1693 & 2580 & 2357 & 295 & 1159 & 1842 & 2314 \\
\hline INPP5F & 278 & 99 & 18 & 23 & 260 & 214 & 383 & 90 \\
\hline PHLDA2 & 20 & 4 & 17 & 15 & 1 & 8 & 5 & 19 \\
\hline SGCE & 856 & 293 & 890 & 730 & 828 & 605 & 761 & 576 \\
\hline
\end{tabular}

\begin{tabular}{|c|c|c|c|c|c|c|c|c|}
\hline & \multicolumn{8}{|c|}{$\begin{array}{l}\text { Muscle (\# of reads containing the SNPs } \\
\text { used to ascribe the allelic expression) }\end{array}$} \\
\hline & \multicolumn{4}{|c|}{ Control } & \multicolumn{4}{|c|}{ LOS } \\
\hline & $\# 1$ & $\# 2$ & $\# 3$ & $\# 4$ & $\# 1$ & $\# 2$ & $\# 3$ & $\# 4$ \\
\hline DIRAS3 & 0 & 0 & 0 & 0 & 0 & 0 & 0 & 0 \\
\hline DLK1 & 5274 & 1868 & 4470 & 4261 & 3925 & 5722 & 7932 & 7287 \\
\hline GNAS & \begin{tabular}{|l|}
1702 \\
\end{tabular} & 1185 & 1780 & \begin{tabular}{|l|}
1350 \\
\end{tabular} & \begin{tabular}{|l|}
1517 \\
\end{tabular} & 2408 & 3476 & 3336 \\
\hline GTL2 & 3663 & 5394 & \begin{tabular}{|l|}
3597 \\
\end{tabular} & \begin{tabular}{|l|}
4144 \\
\end{tabular} & 1348 & 4016 & 5536 & 5248 \\
\hline$H 19$ & 8841 & 4663 & 8245 & \begin{tabular}{|l|}
6604 \\
\end{tabular} & 7834 & 8613 & 32769 & 34196 \\
\hline MAGEL2 & 50 & 31 & 50 & 47 & 97 & 73 & 89 & 119 \\
\hline \begin{tabular}{|l|} 
NAP1L5 \\
\end{tabular} & 59 & 39 & 64 & 48 & 62 & 100 & 71 & 74 \\
\hline NNAT & \begin{tabular}{|l|}
288 \\
\end{tabular} & 59 & 273 & 297 & 116 & 699 & 341 & 1862 \\
\hline PEG10 & 9361 & 7093 & 12023 & 11439 & 9742 & 11932 & 18427 & 12250 \\
\hline PEG3 & 173 & 202 & 341 & 203 & 86 & 293 & \begin{tabular}{|l|}
1457 \\
\end{tabular} & \begin{tabular}{|l|l|}
1972 \\
\end{tabular} \\
\hline PLAGL1 & 881 & 541 & 1056 & 619 & 854 & 1109 & 1700 & 4509 \\
\hline RTL1 & 47 & 37 & 64 & 57 & NA & 72 & 242 & 142 \\
\hline SNRPN & 234 & 101 & 269 & 205 & 397 & 525 & 684 & 730 \\
\hline BEGAIN & 33 & 15 & 21 & 19 & 30 & 31 & 57 & 92 \\
\hline CDKN1C & 603 & 237 & 547 & 436 & 360 & 563 & 970 & 468 \\
\hline IGF2 & 9937 & 10007 & 9942 & 8423 & 8188 & 11964 & 25527 & 23919 \\
\hline IGF2R & \begin{tabular}{|l}
2109 \\
\end{tabular} & 1510 & 2537 & 1989 & \begin{tabular}{|l|}
807 \\
\end{tabular} & 1599 & 1899 & 2887 \\
\hline INPP5F & 593 & 312 & 88 & 71 & 839 & 973 & \begin{tabular}{|l|}
976 \\
\end{tabular} & 121 \\
\hline PHLDA2 & 2 & 1 & 2 & 0 & 1 & 1 & 0 & 6 \\
\hline SGCE & \begin{tabular}{|l|}
872 \\
\end{tabular} & 214 & 841 & 697 & \begin{tabular}{|l|}
694 \\
\end{tabular} & 1197 & 970 & 1014 \\
\hline
\end{tabular}




\begin{tabular}{|c|c|c|c|c|c|c|c|c|c|c|c|c|c|c|c|c|}
\hline & & & & & 34 & nscri & amou & 1110 & \|енс & pre & slon of & the im & pimt & ge & in & 105 \\
\hline & & & $\begin{array}{l}\text { Weight } \rightarrow \\
\text { Fetus } \rightarrow\end{array}$ & $\begin{array}{c}392 \mathrm{~g} \\
\text { Control \#1 }\end{array}$ & $\begin{array}{c}404 \mathrm{~g} \\
\text { Control \#2 }\end{array}$ & $\begin{array}{c}408 \mathrm{~g} \\
\text { Control \#3 }\end{array}$ & $\begin{array}{c}416 \mathrm{~g} \\
\text { Control \#4 }\end{array}$ & & $\begin{array}{c}514 \mathrm{~g} \\
\text { LOS \#1 }\end{array}$ & $\begin{array}{l}518 \mathrm{~g} \\
\text { LOS \#2 }\end{array}$ & $\begin{array}{c}620 \mathrm{~g} \\
\text { LOS \#3 }\end{array}$ & $\begin{array}{l}714 \mathrm{~g} \\
\text { LOS \#4 }\end{array}$ & & Fold C & hange & \\
\hline $\begin{array}{l}\text { Gene \# } \\
1\end{array}$ & $\begin{array}{l}\text { Tissue } \\
\text { Brain }\end{array}$ & $\begin{array}{l}\text { Gene Name } \downarrow \\
\text { AlM1 }\end{array}$ & $\begin{array}{c}\text { Gene ID } \\
\text { ENSBTAG00000017527 }\end{array}$ & 14.92 & $\begin{array}{l}\text { read cour } \\
13.55\end{array}$ & $\begin{array}{l}\text { its/million } \\
10.35\end{array}$ & 27.33 & $\begin{array}{ll}\text { Mean } \\
16.54\end{array}$ & 8.77 & $\begin{array}{c}\text { read cour } \\
11.33\end{array}$ & $\begin{array}{l}\text { nts/million } \\
11.84\end{array}$ & 16.02 & Los \#1 & $\begin{array}{l}\text { LOS \#2 } \\
0.69\end{array}$ & $\begin{array}{c}\text { LOS \#3 } \\
0.72\end{array}$ & $\begin{array}{c}\text { LOS \#4 } \\
0.97\end{array}$ \\
\hline & $\begin{array}{l}\text { Bran } \\
\text { Kidney }\end{array}$ & AIM7 & ENSBTAG00000017527 & $\begin{array}{l}14.92 \\
55.80 \\
\end{array}$ & $\begin{array}{l}13.55 \\
58.91\end{array}$ & $\begin{array}{l}70.35 \\
53.05\end{array}$ & $\begin{array}{l}27.33 \\
53.49\end{array}$ & $\begin{array}{l}1.0 .54 \\
55.31\end{array}$ & 57.90 & 64.92 & 34.21 & 51.56 & \begin{tabular}{|l|l|}
0.03 \\
1.05
\end{tabular} & $\begin{array}{l}0.69 \\
1.17\end{array}$ & 0.62 & 0.93 \\
\hline & $\begin{array}{l}\text { Nover } \\
\text { Liver }\end{array}$ & AIM1 & ENSBTAG00000017527 & $\begin{array}{l}18.72 \\
18.72\end{array}$ & 16.12 & 20.52 & 21.26 & 19.15 & 18.07 & $\begin{array}{l}19.66 \\
19.66\end{array}$ & 17.43 & $\begin{array}{l}17.49 \\
\end{array}$ & 0.94 & 1.03 & 0.91 & 0.91 \\
\hline & Muscle & AIM1 & ENSBTAG000000017527 & 12.09 & 11.55 & 14.03 & 18.61 & 14.07 & 14.43 & 13.37 & 13.65 & 20.54 & 1.03 & 0.95 & 0.97 & 1.46 \\
\hline 2 & Brain & AMPD3 & ENSBTAG00000015821 & 15.17 & 7.10 & 15.79 & 15.46 & 13.38 & 1.61 & 2.05 & 5.93 & 4.23 & 0.12 & 0.15 & 0.44 & 0.32 \\
\hline & $\begin{array}{l}\text { Kidney } \\
\text { Liver } \\
\text { Muscle }\end{array}$ & $\begin{array}{l}\text { AMPD3 } \\
\text { AMPD3 } \\
\text { AMPD3 }\end{array}$ & $\begin{array}{l}\text { ENSBTAG000000015821 } \\
\text { ENSBAGG00000015821 } \\
\text { ENSBTAG000000015821 }\end{array}$ & 4.44 & 4.90 & 5.21 & 4.42 & 4.74 & 5.23 & 3.84 & 5.99 & 6.38 & 1.10 & 0.81 & 1.26 & 1.35 \\
\hline 3 & Brain & ANO1 & ENSBTAG00000009997 & 2.32 & 1.25 & 3.34 & 1.98 & 2.22 & 0.54 & 0.54 & 1.33 & 3.34 & 0.24 & 0.24 & 0.60 & 1.50 \\
\hline & Kidney & ANO1 & ENSBTAG000000099997 & 16.46 & 12.15 & 13.47 & 15.92 & 14.50 & 10.05 & 12.75 & 15.85 & 13.84 & 0.69 & 0.88 & 1.09 & 0.95 \\
\hline & Liver & ANO1 & ENSBTAG00000009997 & 18.56 & 27.72 & 20.46 & 21.36 & 22.02 & 11.50 & 26.11 & 22.92 & 20.59 & 0.52 & 19 & .04 & 0.94 \\
\hline & Muscle & ANO1 & ENSBTAG000000009997 & 2.03 & 2.21 & 2.13 & 3.78 & 2.54 & 1.62 & 2.22 & 2.95 & 8.80 & 0.64 & 0.87 & 1.16 & 3.46 \\
\hline 4 & Brain & ART5 & ENSBTAG00000037826 & & & & & & & & & & & & & \\
\hline & Kidney & $\begin{array}{l}\text { ART5 } \\
\text { ART5 }\end{array}$ & ENSBTAG00000037826 & & & & & & & & & & & & & \\
\hline & $\begin{array}{l}\text { Liver } \\
\text { Muscle }\end{array}$ & $\begin{array}{l}\text { ART5 } \\
\text { ART5 }\end{array}$ & $\begin{array}{l}\text { ENSBATGG00000037826 } \\
\text { ENSBAGG0000037826 }\end{array}$ & $\begin{array}{l}\begin{array}{l}19.53 \\
2.69\end{array} \\
\end{array}$ & $\begin{array}{l}16.85 \\
1.99\end{array}$ & $\begin{array}{l}65.16 \\
1.92\end{array}$ & $\begin{array}{l}21.13 \\
1.74\end{array}$ & $\begin{array}{l}30.67 \\
2.08\end{array}$ & $\begin{array}{l}4.653 \\
2.66\end{array}$ & $\begin{array}{l}68.44 \\
1.16\end{array}$ & $\begin{array}{l}31.52 \\
1.77\end{array}$ & $\begin{array}{l}62.00 \\
1.05\end{array}$ & $\begin{array}{l}1.52 \\
1.28\end{array}$ & $\begin{array}{l}2.23 \\
0.56\end{array}$ & $\begin{array}{l}1.03 \\
0.85\end{array}$ & $\begin{array}{l}2.02 \\
0.50\end{array}$ \\
\hline 5 & Brain & $A S B 4$ & ENSBTAG00000018185 & & & & & & & & & & & & & \\
\hline & $\begin{array}{l}\text { Brain } \\
\text { Kidney }\end{array}$ & $\begin{array}{ll}A S B 4^{2} \\
A S B 4\end{array}$ & ENSBTAG00000018185 & & & & & & & & & & & & & \\
\hline & Liver & $A S B 4$ & ENSBTAG00000018185 & 2.34 & 0.82 & 2.14 & 1.16 & 1.62 & 1.41 & 2.32 & 1.76 & 1.93 & 0.87 & 1.43 & 1.09 & 1.19 \\
\hline & Muscle & ASB4 & ENSBTAG00000018185 & 26.59 & 19.17 & 35.95 & 21.68 & 25.84 & 25.21 & 20.90 & 21.92 & 18.84 & 0.98 & 0.81 & 0.85 & 0.73 \\
\hline 6 & Brain & ATP10A & ENSBTAG00000027081 & 3.70 & 2.88 & 2.18 & 2.94 & 2.92 & 3.12 & 3.02 & 2.95 & 3.65 & 1.07 & 1.03 & 1.01 & 1.25 \\
\hline & $\begin{array}{l}\text { Kidney } \\
\text { Liver }\end{array}$ & $\begin{array}{l}\text { ATP10A } \\
\text { ATP10A }\end{array}$ & $\begin{array}{l}\text { ENSBTAG000000027081 } \\
\text { ENSBATGP00002778 }\end{array}$ & 15.19 & 12.28 & 14.78 & 13.76 & 14.00 & 15.70 & 13.03 & 15.17 & 18.12 & 1.12 & 0.93 & 1.08 & 1.29 \\
\hline & Muscle & ATP10A & ENSBTAG00000027081 & 9.07 & 14.21 & 9.80 & 10.33 & 10.85 & 6.80 & 13.12 & 10.46 & 16.44 & 0.63 & 1.21 & 0.96 & 1.51 \\
\hline 7 & Brain & $A X L$ & ENSBTAG00000003166 & 49.94 & 44.97 & 25.23 & 32.40 & 38.13 & 3.72 & 21.27 & 26.53 & 48.49 & 36 & 56 & .70 & 1.27 \\
\hline & Kidney & $A X L$ & 0003166 & 44.39 & 43.00 & 38.66 & 37.44 & 40.87 & 42.52 & 47.02 & 54.70 & 56.15 & 1.04 & & & \\
\hline & Liver & $A X L$ & ENSBTAGO & 16.94 & 23.3 & 17.79 & 17.64 & 18.92 & 14.32 & 15.81 & 18.12 & 22.38 & 0.76 & 0.84 & 0.96 & 1.18 \\
\hline & Muscle & $A \times L$ & ENSBTAG000000003166 & 56.15 & 81.93 & 50.93 & 60.02 & 62.26 & 67.36 & 60.48 & 61.57 & 86.03 & 1.08 & 0.97 & 0.99 & 1.38 \\
\hline 8 & Brain & BEGAIN & ENSBTAG00000017716 & 39.81 & 53.25 & 48.86 & 45.47 & 46.85 & 20.92 & 28.86 & 34.30 & 52.22 & 0.45 & 62 & 0.73 & 1.11 \\
\hline & Kidney & BEGAIN & ENSBTAG000000017716 & 23.96 & 25.75 & 22.08 & 24.96 & 24.19 & 33.94 & 20.49 & 38.18 & 38.76 & 1.40 & 85 & 1.58 & 1.60 \\
\hline & Liver & BEGAIN & ENSBTAG00000017716 & 10.09 & 20.14 & 13.05 & 11.44 & 13.68 & 4.58 & 8.15 & 16.86 & 14.45 & 0.34 & 60 & 1.23 & 1.06 \\
\hline & Muscle & BEGAIN & ENSBTAG00000017716 & 29.28 & 37.75 & 24.84 & 22.85 & 28.68 & 25.16 & 23.79 & 47.14 & 78.37 & 0.88 & 0.83 & 1.64 & 2.73 \\
\hline 9 & Brair & BLCAP & ENSB & 244.15 & 198.90 & 278.30 & 362.57 & 270.98 & 215.21 & 154.30 & 123.31 & 145.91 & 0.79 & 0.57 & 0.46 & 0.54 \\
\hline & Kidney & BLCAP & 003209 & 59.01 & 60.73 & 59.89 & 60.07 & 59.93 & 59.96 & 63.92 & 87.29 & 67.50 & $\begin{array}{l}.100 \\
1.00\end{array}$ & 1.07 & $\begin{array}{l}0.46 \\
1.46\end{array}$ & $\begin{array}{l}1.13 \\
1.13\end{array}$ \\
\hline & Liver & BLCAP & ENSBTAG00000003209 & 145.28 & 169.40 & 137.41 & 122.22 & 143.58 & 128.22 & 175.99 & 121.96 & 117.55 & 0.89 & 1.23 & 0.85 & 0.82 \\
\hline & Muscle & BLCAP & ENSBTAG00000003209 & 236.38 & 236.13 & 234.50 & 195.04 & 225.51 & 196.76 & 242.64 & 213.36 & 163.29 & 0.87 & 1.08 & 0.95 & 0.72 \\
\hline 10 & $\begin{array}{c}\text { Brain } \\
\text { Kidney }\end{array}$ & $\begin{array}{l}\text { CALCR } \\
\text { CALCR }\end{array}$ & $\begin{array}{l}\text { ENSBTAG000000017448 } \\
\text { ENSBTAG00000017458 }\end{array}$ & 39.69 & 8.49 & 10.31 & 15.19 & 18.42 & 1.29 & 3.91 & 2.95 & 10.04 & 0.07 & 0.21 & 0.16 & 0.55 \\
\hline & $\begin{array}{l}\text { Kidney } \\
\text { Liver }\end{array}$ & $\begin{array}{l}\text { CALCR } \\
\text { CALCR }\end{array}$ & $\begin{array}{l}\text { ENSBBAG00000017458 } \\
\text { ENSBTAG0000017458 }\end{array}$ & & & & & & & & & & & & & \\
\hline & Muscle & CALCR & ENSBTAG00000017458 & 16.48 & 17.93 & 16.99 & 19.84 & 17.81 & 14.03 & 16.75 & 20.05 & 10.44 & 0.79 & 0.94 & 1.13 & 0.59 \\
\hline 11 & Brain & CASD1 & ENSBTAG00000009109 & 115.57 & 122.09 & 169.26 & 179.98 & 146.73 & 104.73 & 146.83 & 110.82 & 101.14 & 0.71 & 1.00 & 0.76 & 0.69 \\
\hline & Kidney & CASD1 & ENSBTAGO & 130.12 & 129.78 & 131.85 & 123.48 & 128.81 & 134.40 & 133.28 & 80.27 & 107.05 & 1.04 & 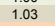 & & \\
\hline & Liver & CASD1 & ENSBTAG00000009109 & 20.18 & 15.80 & 19.37 & 20.68 & 19.01 & 22.30 & 20.69 & 14.41 & 14.61 & 1.17 & 1.09 & 0.76 & 0.77 \\
\hline & Muscle & CASD1 & ENSBTAG00000009109 & 135.21 & 125.17 & 161.12 & 162.94 & 146.11 & 160.18 & 133.00 & 95.40 & 93.44 & 1.10 & 0.91 & 0.65 & 0.64 \\
\hline 12 & $\mathrm{Bra}$ & CD81 & 17495 & 227.68 & 142.42 & 138.33 & 72.39 & 170.21 & 128.99 & 114.46 & 78 & 164.33 & 0.76 & 0.67 & 0.62 & 0.97 \\
\hline & Kidney & CD81 & ENSBTAG00000047495 & 158.59 & 151.67 & 145.15 & 150.57 & 151.49 & \begin{tabular}{|l|}
151.07 \\
\end{tabular} & 158.74 & 302.12 & 192.02 & & & & \\
\hline & Liver & CD81 & ENSBTAG00000047495 & 120.49 & 154.14 & 150.09 & 132.14 & 139.22 & 158.50 & 122.69 & 135.60 & 129.99 & 1.14 & 0.8 & 77 & \\
\hline & Muscle & CD81 & ENSBTAG00000047495 & 213.42 & 176.38 & 183.69 & 182.11 & 188.90 & 205.18 & 213.26 & 202.03 & 237.90 & 1.09 & 1.13 & 1.07 & 1.26 \\
\hline 13 & Brain & CDKN1C & ENSBTAG00000031184 & 16.88 & 19.30 & 8.24 & 17.86 & 15.57 & 8.93 & 8.94 & 21.96 & 17.84 & 0.57 & 0.57 & 1.41 & 1.15 \\
\hline & Kidney & CDKN1C & ENSBTAG00000031184 & 98.97 & 87.04 & 86.17 & & 92.78 & 50.68 & 90.01 & 208.35 & & 0 & & 5 & \\
\hline & Liver & CDKN1C & ENSBTAG00000031184 & 51.32 & & 61.21 & & & & & 103.09 & & & & & \\
\hline & Muscle & CDKN1C & ENSBTAG00000031184 & 189.34 & 252.95 & 152.50 & 134.21 & 182.25 & 130.93 & 150.49 & 278.60 & 143.77 & 0.72 & 0.83 & 1.53 & 0.79 \\
\hline 14 & Brair & CMAH & 3892 & & & & & & & & & & & & & \\
\hline & Kidney & CMAH & ENSBTAG00000003892 & 2.37 & & 37 & 315 & & 1.22 & & & & 0.61 & (1) & 72 & 0.88 \\
\hline & $\begin{array}{l}\text { Liver } \\
\text { Muscle }\end{array}$ & $\begin{array}{l}\text { CMAH } \\
\text { CMAH }\end{array}$ & $\begin{array}{l}\text { ENSBTAG00000003892 } \\
\text { ENSBTAG00000003892 }\end{array}$ & 39.89 & 32.27 & 37.36 & 34.50 & 36.01 & 41.02 & 40.14 & 36.64 & 37.64 & 1.14 & 1.11 & 1.02 & 1.05 \\
\hline 15 & Brain & CNTN3 & & 20.74 & 15.76 & 28.53 & 37.6 & 25.67 & 59.65 & 24.10 & 17.27 & 39 & & & & 40 \\
\hline & Kidney & CNTN3 & ENSBTAG000000007615 & 6.93 & 7.57 & 10.32 & 6.41 & 7.81 & 7.50 & 6.67 & 5.13 & 9.08 & 0.96 & 0.85 & 0.66 & 1.16 \\
\hline & $\begin{array}{l}\text { Liver } \\
\text { Muscle }\end{array}$ & $\begin{array}{l}\text { CNTN3 } \\
\text { CNTN3 }\end{array}$ & $\begin{array}{l}\text { ENSBBAG } \\
\text { ENSBTA }\end{array}$ & 44.35 & 38.68 & 41.83 & 53.32 & 44.55 & 57.56 & 55.00 & 32.99 & 23.45 & 1.29 & 1.23 & 0.74 & 0.53 \\
\hline 16 & $\mathrm{Br}$ & COBL & 3806 & 11.71 & 23.73 & 15.97 & 9.51 & 15.23 & 42.87 & 24.18 & 57 & 21.16 & 2. & 1.59 & 3.76 & 1.39 \\
\hline & Kidney & COBL & ENSBTAC & 32 & 36 & 30 & & & & & 30. & & 1. & & & \\
\hline & Liver & $C O B L$ & ENSBTAG00000023806 & 7.91 & 11.54 & $\begin{array}{c}6.72 \\
165.52\end{array}$ & 10.37 & $\begin{array}{c}9.13 \\
91575\end{array}$ & 8.02 & 7.82 & 8.90 & 9.50 & 0.88 & 0.8 & & 0 \\
\hline & Muscle & COBL & ENSBTAG00000023806 & 162.94 & 148.27 & 165.52 & 142.28 & 154.75 & 173.62 & 141.27 & 149.05 & 97.72 & 1.12 & 0.91 & 0.96 & 0.63 \\
\hline 17 & $\mathrm{Br}$ & MMD1 & & 23.50 & & & & 27.45 & 23.08 & 27. & 23.48 & 22.75 & 0.84 & 1.8 & 0.86 & 0.83 \\
\hline & Kid & MMD1 & EN & & & & & & & & 68 & & & & & \\
\hline & Liver & COMMD1 & & 18 & & 14 & & 16.00 & 22 & 18 & 14. & & 1.43 & 1.13 & & 0 \\
\hline & Muscle & COMMD1 & ENSBTAG000000019867 & 54.97 & 36.91 & 55.81 & 50.10 & 49.45 & 62.39 & 57.53 & 36.15 & 35.62 & 1.26 & 1.16 & 0.73 & 0.72 \\
\hline 18 & Brain & COPG2 & EI & 43.1 & 43 & 45. & 56.79 & 47 & 56 & 50.67 & 43 & 35 & 1. & 1.07 & 0.92 & 0.75 \\
\hline & & $c c$ & & & & & & & & & & & & & & \\
\hline & Liver & COPG2 & EN & & 9 & 9 & & & & 12. & 10 & & 1. & & & \\
\hline & Muscle & COPG2 & ENSBTAG00000017245 & 41.56 & 35.4 & 46.53 & 43.16 & 41.66 & 47.02 & 46.59 & 37.57 & 25.24 & 1.13 & 1.12 & 0.90 & 0.61 \\
\hline 19 & $\mathrm{Br}$ & & & & & & & & & & & & & & & \\
\hline & & & & & & & & & & & & & & & & \\
\hline & Liv & CPA & EN & 1.62 & 2 & 1. & & & 0 & 1. & 1. & & 0. & & & \\
\hline & Muscle & CPA4 & ENSBTAG00000046648 & 95.97 & 114.68 & 88.19 & 107.26 & 101.52 & 51.95 & 99.51 & 61.02 & 38.67 & 0.51 & 0.98 & 0.60 & 0.38 \\
\hline 20 & $\mathrm{Bra}$ & $D C$ & E & & 45.7 & 21. & & 50. & 27. & 65. & & & 0.55 & 1.30 & 125 & 1.18 \\
\hline & Kid & $D C$ & & & & & & & & & & & & & & \\
\hline & Liv & $D C N$ & ENSBTAG & 79.32 & 71 & & & 73.44 & 92.84 & & 74. & 94.42 & $1.2 \mathrm{Y}$ & 0.9 & & \\
\hline & Muscle & $D C N$ & ENSBTAG000000003505 & 1788.34 & 1253.83 & 1724.73 & 2079.08 & 1711.50 & 3496.55 & 2021.10 & 1847.74 & 1859.22 & 2.04 & 1.18 & 1.08 & 1.09 \\
\hline 21 & & & & & & & & & & & & & & & & \\
\hline & & & & & & & & & & & & & & & & \\
\hline & $\begin{array}{l}\text { Liver } \\
\text { Muscle }\end{array}$ & $\begin{array}{l}D D C \\
D D C\end{array}$ & $\begin{array}{l}\text { ENSBTAG000000020869 } \\
\text { ENSBTAG00000020869 }\end{array}$ & 14.73 & 20 & 17. & & & 19 & 12 & 19. & & 1.08 & 71 & 1.10 & 1.26 \\
\hline 22 & Brai & & & 1357 & & & & & 12 & & 118 & & & 10 & 1. & 0 \\
\hline & Kid & & & & & & & & & 36. & & & $\begin{array}{l}1.10 \\
0.85\end{array}$ & & 1.00 & 0.78 \\
\hline & Liver & DHCR7 & ENSBTAG000000016465 & 93 & 111 & 94. & 76. & 94.05 & 99.1 & 82.22 & 157.78 & 116.83 & 1.05 & 0.87 & 1. & 1.24 \\
\hline & Muscle & DHCR7 & ENSBTAG00000016465 & 27.91 & 39.30 & 21.83 & 19.53 & 27.14 & 22.36 & 21.57 & 28.27 & 35.02 & 0.82 & 0.79 & 1.04 & 1.29 \\
\hline
\end{tabular}




\begin{tabular}{|c|c|c|c|c|c|c|c|c|c|c|c|c|c|c|c|c|c|c|c|c|c|}
\hline \multirow{2}{*}{$\begin{array}{c}\text { Gene \# } \\
23\end{array}$} & \multirow{2}{*}{$\begin{array}{l}\text { Tissue } \\
\text { Brain } \\
\text { Kidney } \\
\text { Liver } \\
\text { Muscle }\end{array}$} & \multirow{2}{*}{$\begin{array}{c}\text { Gene Name } \downarrow \\
\text { DIO3 } \\
\text { DIO3 } \\
\text { DIO3 } \\
\text { DIO3 }\end{array}$} & \multirow{2}{*}{$\begin{array}{l}\text { Weight } \rightarrow \\
\text { Fetus } \rightarrow \\
\text { Gene ID } \downarrow \\
\text { ENSBTAG00000043578 }\end{array}$} & \multirow{2}{*}{ 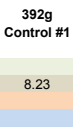 } & \multirow{2}{*}{\multicolumn{2}{|c|}{$\begin{array}{cc}\begin{array}{cc}404 \mathrm{~g} & 408 \mathrm{~g} \\
\text { Control \#2 Control \#3 } \\
\text { read counts/million }\end{array} \\
6.76 \quad 9.40 \\
6.76\end{array}$}} & $\stackrel{416 \mathrm{~g}}{\text { Control \#4 }}$ & \multirow{2}{*}{\begin{tabular}{|c|} 
Mean \\
7.95 \\
\end{tabular}} & \multirow{2}{*}{]$_{7.64}^{\begin{array}{c}514 \mathrm{~g} \\
\text { LOS \#1 }\end{array}}$} & \multirow{2}{*}{$\begin{array}{c}518 \mathrm{~g} \\
\text { LOS \#2 } \\
\text { read count } \\
7.43\end{array}$} & \multirow{2}{*}{ 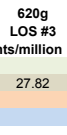 } & \multirow{2}{*}{$\begin{array}{c}714 \mathrm{~g} \\
\text { LOS \#4 } \\
9.94\end{array}$} & \multicolumn{4}{|c|}{$\begin{array}{c}\text { Fold Change } \\
\text { LOS \#1 LOS \#2 LOS \#3 LOS \#4 }\end{array}$} & \multicolumn{4}{|c|}{\begin{tabular}{|c|} 
FDR (False Discovery Rate) \\
LOS \#1 LOS \#2 LOS \#3 LOS \#4
\end{tabular}} & \multirow[t]{2}{*}{$\begin{array}{c}\text { Alllelic } \\
\text { Exrssion } \\
\text { in Control } \\
\text { E NA }\end{array}$} \\
\hline & & & & & & & 7.42 & & & & & & 0.96 & 0.93 & 3.50 & 1.25 & 1.00 & 1.00 & 0.00 & 0.66 & \\
\hline 24 & $\begin{array}{l}\text { Brain } \\
\text { Kidney } \\
\text { Liver } \\
\text { Muscle }\end{array}$ & $\begin{array}{l}\text { DIRAS3 } \\
\text { DIRAS3 } \\
\text { DIRAS3 } \\
\text { DIRAS3 }\end{array}$ & $\begin{array}{l}\text { ENSBTAG000000012182 } \\
\text { ENSBTAG000000012182 } \\
\text { ENSBATAG000000012182 } \\
\text { ENSBTAG00000012182 }\end{array}$ & $\begin{array}{c}676.74 \\
19.17\end{array}$ & $\begin{array}{l}254.81 \\
28.65\end{array}$ & $\begin{array}{l}459.65 \\
27.23\end{array}$ & $\begin{array}{c}688.40 \\
15.38\end{array}$ & $\begin{array}{c}519.90 \\
22.61\end{array}$ & $\begin{array}{l}209.89 \\
29.93\end{array}$ & $\begin{array}{c}146.21 \\
22.66\end{array}$ & $\begin{array}{l}221.82 \\
63.75\end{array}$ & $\begin{array}{r}283.04 \\
37.27\end{array}$ & $\begin{array}{l}0.40 \\
1.32\end{array}$ & $\begin{array}{l}0.28 \\
1.00\end{array}$ & $\begin{array}{l}0.43 \\
2.82\end{array}$ & $\begin{array}{l}0.54 \\
1.65\end{array}$ & $\begin{array}{l}0.01 \\
1.00\end{array}$ & $\begin{array}{l}0.00 \\
1.00\end{array}$ & $\begin{array}{l}0.03 \\
0.00\end{array}$ & $\begin{array}{l}0.17 \\
0.13\end{array}$ & $\begin{array}{l}\text { Paternal } \\
\text { Paternal }\end{array}$ \\
\hline 25 & $\begin{array}{l}\text { Brain } \\
\text { Kidney } \\
\text { Liver } \\
\text { Muscle }\end{array}$ & $\begin{array}{l}\text { DLGAP2 } \\
\text { DLGAP2 } \\
\text { DLGAP2 } \\
\text { DLGAP2 }\end{array}$ & $\begin{array}{l}100847272 \\
100847272 \\
1008477272 \\
100847272\end{array}$ & 17.61 & 32.88 & 32.78 & 24.15 & 26.85 & 42.33 & 27.31 & 37.75 & 31.39 & 1.58 & 1.02 & 1.41 & 1.17 & 0.38 & 1.00 & 0.57 & 0.93 & NA \\
\hline 26 & $\begin{array}{l}\text { Brain } \\
\text { Kidney } \\
\text { Liver } \\
\text { Muscle }\end{array}$ & $\begin{array}{l}\text { DLK1 } \\
D L K 1 \\
D L K 1 \\
D L K 1\end{array}$ & $\begin{array}{l}\text { ENSBTAG000000037899 } \\
\text { ENSBTAG00000037899 } \\
\text { ENSBATAG000000037899 } \\
\text { ENSBTAG000000037899 }\end{array}$ & $\begin{array}{l}13.34 \\
485.84 \\
834.07 \\
1367.87\end{array}$ & $\begin{array}{r}12.85 \\
478.26 \\
109.22 \\
1637.22\end{array}$ & $\begin{array}{c}4.61 \\
505.79 \\
893.59 \\
1103.25\end{array}$ & \begin{tabular}{r|}
14.13 \\
543.00 \\
808.18 \\
1176.30
\end{tabular} & $\begin{array}{c}11.23 \\
503.22 \\
906.76 \\
1321.16\end{array}$ & $\begin{array}{c}2.10 \\
605.83 \\
979.05 \\
1118.00\end{array}$ & $\begin{array}{c}2.17 \\
561.60 \\
702.84 \\
1263.38\end{array}$ & $\begin{array}{c}2.88 \\
1929.80 \\
1058.41 \\
1827.77\end{array}$ & $\begin{array}{r}38.61 \\
714.54 \\
945.46 \\
1521.74\end{array}$ & $\begin{array}{l}0.19 \\
1.20 \\
1.08 \\
0.85\end{array}$ & $\begin{array}{l}0.19 \\
1.12 \\
0.78 \\
0.96\end{array}$ & $\begin{array}{l}0.26 \\
3.83 \\
1.17 \\
1.38\end{array}$ & $\begin{array}{l}3.44 \\
1.42 \\
1.04 \\
1.15\end{array}$ & $\begin{array}{l}0.05 \\
0.76 \\
0.33 \\
0.73\end{array}$ & $\begin{array}{l}0.11 \\
1.00 \\
0.75 \\
1.00\end{array}$ & $\begin{array}{l}0.18 \\
0.00 \\
0.16 \\
0.06\end{array}$ & $\begin{array}{l}0.08 \\
0.01 \\
0.53 \\
0.32\end{array}$ & $\begin{array}{l}\text { Paternal } \\
\text { Patemal } \\
\text { Patemal } \\
\text { Paternal }\end{array}$ \\
\hline 27 & $\begin{array}{l}\text { Brain } \\
\text { Kidney } \\
\text { Liver } \\
\text { Muscle }\end{array}$ & $\begin{array}{l}D L X 5 \\
D L X 5 \\
D L X 5 \\
D L X 5\end{array}$ & $\begin{array}{l}\text { ENSBTAG000000018645 } \\
\text { ENSBTAG000000018645 } \\
\text { ENSBATAG000000018645 } \\
\text { ENSBTAG000000018645 }\end{array}$ & 0.94 & 9.70 & 13.07 & 0.99 & 6.17 & 3.33 & 3.79 & 10.17 & 6.99 & 0.54 & 0.61 & 1.65 & 1.13 & 0.85 & 1.00 & 0.72 & 0.96 & NA \\
\hline 28 & $\begin{array}{l}\text { Brain } \\
\text { Kidney } \\
\text { Liver } \\
\text { Muscle }\end{array}$ & $\begin{array}{l}\text { DNMT1 } \\
\text { DNMT1 } \\
\text { DNMT1 } \\
\text { DNMT1 }\end{array}$ & $\begin{array}{l}\text { ENSBTAG000000002736 } \\
\text { ENSBTAG000000002736 } \\
\text { ENSBATGG000000002736 } \\
\text { ENSBTAG000000002736 }\end{array}$ & $\begin{array}{l}80.07 \\
98.62 \\
113.14 \\
69.85\end{array}$ & $\begin{array}{c}91.01 \\
94.18 \\
125.51 \\
91.31\end{array}$ & $\begin{array}{l}69.29 \\
100.19 \\
110.20 \\
67.66\end{array}$ & $\begin{array}{c}76.29 \\
92.28 \\
108.23 \\
71.37\end{array}$ & $\begin{array}{l}79.17 \\
96.32 \\
114.27 \\
75.05\end{array}$ & $\begin{array}{l}84.72 \\
95.58 \\
78.12 \\
60.76\end{array}$ & $\begin{array}{c}82.66 \\
90.64 \\
126.09 \\
79.17\end{array}$ & $\begin{array}{l}86.36 \\
69.96 \\
135.13 \\
100.73\end{array}$ & $\begin{array}{c}93.53 \\
94.35 \\
131.78 \\
107.51\end{array}$ & $\begin{array}{l}1.07 \\
0.99 \\
0.68 \\
0.81\end{array}$ & $\begin{array}{l}1.04 \\
0.94 \\
1.10 \\
1.05\end{array}$ & $\begin{array}{l}1.09 \\
0.73 \\
1.18 \\
1.34\end{array}$ & 1.43 & $\begin{array}{l}0.90 \\
1.00 \\
0.00 \\
0.45\end{array}$ & $\begin{array}{l}1.00 \\
1.00 \\
0.79 \\
0.99\end{array}$ & $\begin{array}{l}0.86 \\
0.00 \\
0.10 \\
0.01\end{array}$ & $\begin{array}{l}0.91 \\
0.97 \\
0.13 \\
0.00\end{array}$ & $\begin{array}{l}\text { Biallelic } \\
\text { Biallelic } \\
\text { Biallelic } \\
\text { Biallelic }\end{array}$ \\
\hline 29 & $\begin{array}{l}\text { Brain } \\
\text { Kidney } \\
\text { Liver } \\
\text { Muscle }\end{array}$ & $\begin{array}{l}\text { DRD 1A } \\
D R D 1 A \\
D R D 1 A \\
D R D 1 A\end{array}$ & $\begin{array}{l}\text { ENSBTAG000000047719 } \\
\text { ENSBAAG000000477719 } \\
\text { ENSBATAG000000047779 } \\
\text { ENSBTAG000000477719 }\end{array}$ & 7.28 & 13.51 & 35.83 & 13.65 & 17.57 & 73.37 & 13.81 & 30.15 & 13.98 & 4.18 & 0.79 & 1.72 & 0.80 & 0.03 & 0.98 & 0.70 & 0.92 & NA \\
\hline 30 & $\begin{array}{l}\text { Brain } \\
\text { Kidney } \\
\text { Liver } \\
\text { Muscle }\end{array}$ & $\begin{array}{l}F B \times O 40 \\
F B X 040 \\
F B X 040 \\
F B \times 040\end{array}$ & $\begin{array}{l}\text { ENSBTAG000000014508 } \\
\text { ENSBTAG000000014508 } \\
\text { ENSBTAG000000014508 } \\
\text { ENSBTAG00000014508 }\end{array}$ & $\begin{array}{l}28.13 \\
4.01\end{array}$ & $\begin{array}{r}23.55 \\
3.72\end{array}$ & $\begin{array}{l}25.98 \\
3.22\end{array}$ & $\begin{array}{r}28.77 \\
3.48\end{array}$ & $\begin{array}{c}26.61 \\
3.61\end{array}$ & $\begin{array}{r}29.96 \\
1.08\end{array}$ & $\begin{array}{l}25.77 \\
0.84\end{array}$ & $\begin{array}{l}9.72 \\
1.32\end{array}$ & $\begin{array}{c}28.68 \\
1.61\end{array}$ & $\begin{array}{l}1.13 \\
0.30\end{array}$ & $\begin{array}{l}0.97 \\
0.23\end{array}$ & $\begin{array}{l}0.37 \\
0.37\end{array}$ & $\begin{array}{l}1.08 \\
0.45\end{array}$ & $\begin{array}{l}1.00 \\
0.00\end{array}$ & $\begin{array}{l}1.00 \\
0.00\end{array}$ & $\begin{array}{l}0.00 \\
0.02\end{array}$ & $\begin{array}{l}0.85 \\
0.02\end{array}$ & NA \\
\hline 31 & $\begin{array}{l}\text { Brain } \\
\text { Kidney } \\
\text { Liver } \\
\text { Muscle }\end{array}$ & $\begin{array}{l}\text { GATM } \\
\text { GATM } \\
\text { GATM } \\
\text { GATM }\end{array}$ & $\begin{array}{l}\text { ENSBTAG000000005586 } \\
\text { ENSBTAG00000005586 } \\
\text { ENSBATGO00000005556 } \\
\text { ENSBTAG00000005586 }\end{array}$ & $\begin{array}{c}191.12 \\
406.54 \\
1228.00 \\
17.66\end{array}$ & $\begin{array}{c}91.15 \\
375.18 \\
943.43 \\
11.15\end{array}$ & $\begin{array}{l}108.32 \\
380.69 \\
1081.66 \\
17.34\end{array}$ & $\begin{array}{c}101.40 \\
347.31 \\
1223.62 \\
14.72\end{array}$ & $\begin{array}{c}123.00 \\
377.43 \\
1119.18 \\
15.22\end{array}$ & $\begin{array}{c}123.99 \\
450.09 \\
1834.72 \\
14.48\end{array}$ & $\begin{array}{c}119.18 \\
331.96 \\
1426.25 \\
14.11\end{array}$ & $\begin{array}{c}81.65 \\
219.69 \\
1036.80 \\
12.67\end{array}$ & $\begin{array}{r}70.08 \\
466.10 \\
1110.94 \\
7.27\end{array}$ & $\begin{array}{l}1.01 \\
1.19 \\
1.64 \\
0.95\end{array}$ & $\begin{array}{l}0.97 \\
0.88 \\
1.27 \\
0.93\end{array}$ & $\begin{array}{l}0.66 \\
0.58 \\
0.93 \\
0.83\end{array}$ & & $\begin{array}{l}0.91 \\
0.86 \\
0.00 \\
0.61\end{array}$ & $\begin{array}{l}1.00 \\
1.00 \\
0.72 \\
1.00\end{array}$ & $\begin{array}{l}0.46 \\
0.00 \\
0.59 \\
0.32\end{array}$ & $\begin{array}{l}0.38 \\
0.24 \\
0.84 \\
0.00\end{array}$ & $\begin{array}{l}\text { Biallelic } \\
\text { Biallelic } \\
\text { Biallelic } \\
\text { Biallelic }\end{array}$ \\
\hline 32 & $\begin{array}{l}\text { Brain } \\
\text { Kidney } \\
\text { Liver } \\
\text { Muscle }\end{array}$ & $\begin{array}{l}\text { GDAP1L1 } \\
\text { GDAPL1L1 } \\
\text { GDAPL1 } \\
\text { GDAP1L1 }\end{array}$ & $\begin{array}{l}\text { ENSBTAG000000002593 } \\
\text { ENSBTAG000000002593 } \\
\text { ENSBATAG00000000253 } \\
\text { ENSBTAG000000002593 }\end{array}$ & 53.31 & 63.78 & 64.79 & 63.36 & 61.31 & 96.71 & 75.97 & 78.22 & 64.26 & 1.58 & 1.24 & 1.28 & 1.05 & 0.32 & 0.97 & 0.70 & 0.99 & Biallelic \\
\hline 33 & $\begin{array}{l}\text { Brain } \\
\text { Kidney } \\
\text { Liver } \\
\text { Muscle }\end{array}$ & $\begin{array}{l}\text { GLIS3 } \\
\text { GLIS3 } \\
\text { GLLS3 } \\
\text { GLIS3 }\end{array}$ & $\begin{array}{l}\text { ENSBTAG000000014467 } \\
\text { ENSBTAG000000014467 } \\
\text { ENSBTAG000000014467 } \\
\text { ENSBTAG00000014467 }\end{array}$ & $\begin{array}{l}10.17 \\
12.02 \\
1.70\end{array}$ & $\begin{array}{c}6.72 \\
14.39 \\
3.63\end{array}$ & $\begin{array}{l}2.69 \\
10.06 \\
2.92\end{array}$ & $\begin{array}{r}5.51 \\
11.77 \\
2.10\end{array}$ & $\begin{array}{l}6.27 \\
12.06 \\
2.59\end{array}$ & $\begin{array}{r}3.12 \\
11.93 \\
2.86\end{array}$ & $\begin{array}{l}3.64 \\
10.48 \\
2.43\end{array}$ & $\begin{array}{l}3.19 \\
1.89 \\
3.58\end{array}$ & $\begin{array}{l}7.65 \\
8.80 \\
5.60\end{array}$ & $\begin{array}{l}0.50 \\
0.99\end{array}$ & $\begin{array}{l}0.87 \\
0.94\end{array}$ & $\begin{array}{l}0.51 \\
0.16 \\
1.39\end{array}$ & 73 & $\begin{array}{l}0.58 \\
1.00 \\
0.63\end{array}$ & $\begin{array}{l}0.94 \\
1.00 \\
1.00\end{array}$ & $\begin{array}{l}0.65 \\
0.00 \\
0.28\end{array}$ & $\begin{array}{l}0.96 \\
0.49 \\
0.01\end{array}$ & NA \\
\hline 34 & $\begin{array}{l}\text { Brain } \\
\text { Kidney } \\
\text { Liver } \\
\text { Muscle }\end{array}$ & $\begin{array}{l}\text { GNAS } \\
\text { GNAS } \\
\text { GNAS } \\
\text { GNAS }\end{array}$ & $\begin{array}{l}\text { ENSBTAG000000017475 } \\
\text { ENSBTAG00000017475 } \\
\text { ENSBAGG000000017745 } \\
\text { ENSBTAG000000174775 }\end{array}$ & $\begin{array}{l}2080.31 \\
2550.90 \\
1038.77 \\
3009.67\end{array}$ & $\begin{array}{l}1323.53 \\
2332.40 \\
1078.07 \\
2521.03\end{array}$ & $\begin{array}{l}1991.16 \\
2537.77 \\
1102.19 \\
2774.96\end{array}$ & $\begin{array}{l}2068.06 \\
2283.96 \\
1040.60 \\
2665.60\end{array}$ & $\begin{array}{l}1865.77 \\
2426.26 \\
1064.91 \\
2742.81\end{array}$ & $\begin{array}{l}1859.64 \\
2584.77 \\
1600.39 \\
3504.88\end{array}$ & $\begin{array}{l}1440.89 \\
2643.09 \\
924.31 \\
3111.15\end{array}$ & $\begin{array}{l}1221.72 \\
4547.49 \\
110.26 \\
3115.71\end{array}$ & $\begin{array}{l}1465.72 \\
3119.53 \\
985.37 \\
3088.79\end{array}$ & $\begin{array}{l}1.00 \\
1.07 \\
1.50 \\
1.28\end{array}$ & $\begin{array}{l}0.77 \\
1.09 \\
0.87 \\
1.13\end{array}$ & $\begin{array}{l}0.65 \\
1.87 \\
1.04 \\
1.14\end{array}$ & & $\begin{array}{l}0.88 \\
1.00 \\
0.00 \\
0.29\end{array}$ & $\begin{array}{l}0.83 \\
1.00 \\
0.92 \\
1.00\end{array}$ & $\begin{array}{l}0.43 \\
0.00 \\
0.86 \\
0.73\end{array}$ & $\begin{array}{l}0.77 \\
0.12 \\
0.77 \\
0.78\end{array}$ & $\begin{array}{l}\text { Maternal } \\
\text { Maternal } \\
\text { Maternal } \\
\text { Maternal }\end{array}$ \\
\hline 35 & $\begin{array}{l}\text { Brain } \\
\text { Kidney } \\
\text { Liver } \\
\text { Muscle }\end{array}$ & $\begin{array}{l}\text { GPR1 } \\
\text { GPR1 } \\
\text { GPR1 } \\
\text { GPR1 }\end{array}$ & $\begin{array}{l}\text { ENSBTAG000000017043 } \\
\text { ENSBTAG000000017043 } \\
\text { ENSBATAG0000000170043 } \\
\text { ENSBTAG000000017043 }\end{array}$ & 0.49 & 0.83 & 0.58 & 0.86 & 0.69 & 0.22 & 2.01 & 0.81 & 14.70 & 0.31 & 2.92 & 1.18 & 21.35 & 0.41 & 0.32 & 0.90 & 0.00 & NA \\
\hline 36 & $\begin{array}{l}\text { Brain } \\
\text { Kidney } \\
\text { Liver } \\
\text { Muscle }\end{array}$ & $\begin{array}{l}\text { GRB10 } \\
\text { GRB10 } \\
\text { GRB10 } \\
\text { GRB10 }\end{array}$ & $\begin{array}{l}\text { ENSBTAG000000017086 } \\
\text { ENSBTAG00000017086 } \\
\text { ENSBATGG000000177866 } \\
\text { ENSBTAG00000017086 }\end{array}$ & $\begin{array}{l}18.26 \\
28.97 \\
37.43 \\
37.64\end{array}$ & $\begin{array}{l}23.73 \\
29.07 \\
42.72 \\
40.81\end{array}$ & $\begin{array}{l}21.31 \\
28.64 \\
41.16 \\
32.24\end{array}$ & $\begin{array}{l}30.48 \\
29.58 \\
38.32 \\
38.09\end{array}$ & $\begin{array}{l}23.44 \\
29.06 \\
40.04 \\
37.20\end{array}$ & $\begin{array}{l}35.82 \\
33.21 \\
42.65 \\
30.97\end{array}$ & $\begin{array}{l}25.61 \\
27.91 \\
41.71 \\
35.12\end{array}$ & $\begin{array}{l}35.73 \\
36.47 \\
49.72 \\
44.87\end{array}$ & $\begin{array}{l}31.86 \\
32.30 \\
47.59 \\
46.06\end{array}$ & $\begin{array}{l}1.53 \\
1.14 \\
1.07 \\
0.83\end{array}$ & 0.94 & 1.21 & & 0.48 & $\begin{array}{l}1.00 \\
1.00\end{array}$ & $\begin{array}{l}0.56 \\
0.32 \\
0.12 \\
0.25\end{array}$ & $\begin{array}{l}0.88 \\
0.72 \\
0.20 \\
0.18\end{array}$ & $\begin{array}{l}\text { Biallelic } \\
\text { Biallelic } \\
\text { Biallelic } \\
\text { Biallelic }\end{array}$ \\
\hline 37 & $\begin{array}{l}\text { Brain } \\
\text { Kidney } \\
\text { Liver } \\
\text { Muscle }\end{array}$ & $\begin{array}{l}\text { GTL2 } \\
\text { GTL2 } \\
\text { GTL2 } \\
\text { GTL2 }\end{array}$ & $\begin{array}{l}100335527 \\
100335527 \\
100335527 \\
100335527\end{array}$ & $\begin{array}{l}2942.28 \\
2743.66 \\
173.71 \\
8618.49\end{array}$ & $\begin{array}{l}3185.48 \\
2951.02 \\
2498.12 \\
12651.22\end{array}$ & $\begin{array}{l}2997.76 \\
2688.35 \\
1587.00 \\
7314.21\end{array}$ & $\begin{array}{r}2675.69 \\
2852.13 \\
1992.35 \\
10408.91\end{array}$ & \begin{tabular}{|l|}
2950.30 \\
2808.79 \\
1953.82 \\
9748.21
\end{tabular} & \begin{tabular}{|l|}
1974.43 \\
3404.54 \\
778.59 \\
3701.30
\end{tabular} & $\begin{array}{l}2183.02 \\
2508.46 \\
1696.79 \\
7428.26\end{array}$ & $\begin{array}{l}1977.22 \\
1347.47 \\
1926.90 \\
6869.11\end{array}$ & $\begin{array}{l}2706.53 \\
3304.71 \\
2177.65 \\
8211.05\end{array}$ & 0.67 & 0.76 & $\begin{array}{l}0.99 \\
0.70\end{array}$ & & 0.00 & $\begin{array}{l}0.93 \\
1.00 \\
1.00 \\
1.00\end{array}$ & $\begin{array}{l}0.63 \\
0.00 \\
0.74 \\
0.54\end{array}$ & $\begin{array}{l}0.97 \\
0.40 \\
0.32 \\
0.80\end{array}$ & $\begin{array}{l}\text { Maternal } \\
\text { Maternal } \\
\text { Maternal } \\
\text { Maternal }\end{array}$ \\
\hline 38 & $\begin{array}{l}\text { Brain } \\
\text { Kidney } \\
\text { Liver } \\
\text { Muscle }\end{array}$ & $\begin{array}{l}H 13 \\
H 13 \\
H 13 \\
H 13\end{array}$ & $\begin{array}{l}\text { ENSBTAG000000008880 } \\
\text { ENSBAAG0000000088480 } \\
\text { ENSBTAG00000008840 } \\
\text { ENSBTAG000000088440 }\end{array}$ & $\begin{array}{l}57.14 \\
47.37 \\
38.30\end{array}$ & $\begin{array}{l}57.48 \\
57.02 \\
43.42\end{array}$ & $\begin{array}{l}28.97 \\
58.03 \\
42.53 \\
35.29\end{array}$ & $\begin{array}{l}65.23 \\
46.39 \\
33.85\end{array}$ & $\begin{array}{l}35.03 \\
59.47 \\
48.33 \\
37.71\end{array}$ & 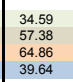 & 41.03 & $\begin{array}{l}92.60 \\
56.86 \\
50.35\end{array}$ & 51.18 & 1.05 & 1.09 & 1.34 & & 0.62 & & & $\begin{array}{l}0.96 \\
0.85 \\
0.13 \\
0.03\end{array}$ & $\mathrm{NA}$ \\
\hline 39 & $\begin{array}{l}\text { Brain } \\
\text { Kidney } \\
\text { Liver } \\
\text { Muscle }\end{array}$ & $\begin{array}{l}\text { H19 } \\
\text { H19 } \\
\text { H19 } \\
\text { H19 }\end{array}$ & $\begin{array}{l}100126192 \\
100126192 \\
100126192 \\
100126192\end{array}$ & $\begin{array}{c}41.91 \\
13054.53 \\
5592.22 \\
9301.91\end{array}$ & $\begin{array}{c}77.74 \\
12685.42 \\
7271.85 \\
10067.82\end{array}$ & $\begin{array}{c}18.75 \\
13964.67 \\
5284.72 \\
8241.40\end{array}$ & $\begin{array}{c}29.50 \\
16180.77 \\
6196.11 \\
7887.78\end{array}$ & \begin{tabular}{|c|}
41.97 \\
13971.35 \\
6086.23 \\
8874.73
\end{tabular} & \begin{tabular}{|c}
17.94 \\
13530.67 \\
5676.19 \\
10559.28
\end{tabular} & $\begin{array}{c}24.10 \\
13092.79 \\
5487.67 \\
9824.36\end{array}$ & $\begin{array}{c}48.16 \\
21343.08 \\
6388.35 \\
9413.54\end{array}$ & $\begin{array}{c}118.40 \\
13285.92 \\
6632.67 \\
9083.55\end{array}$ & 1.19 & 1.11 & 1.06 & 1.02 & 41 & 1.00 & $\begin{array}{l}0.67 \\
0.75\end{array}$ & $\begin{array}{l}0.00 \\
0.91 \\
0.55 \\
0.77\end{array}$ & $\begin{array}{l}\text { Maternal } \\
\text { Maternal } \\
\text { Maternal } \\
\text { Maternal }\end{array}$ \\
\hline 40 & $\begin{array}{l}\text { Brain } \\
\text { Kidney } \\
\text { Liver } \\
\text { Muscle }\end{array}$ & $\begin{array}{l}\text { HTR2A } \\
\text { HTR2A } \\
\text { HTR2A } \\
\text { HTR2A }\end{array}$ & $\begin{array}{l}\text { ENSBTAG000000013498 } \\
\text { ENSBAGG000000013498 } \\
\text { ENSBTAG000000013498 } \\
\text { ENSBTAG00000013498 }\end{array}$ & $\begin{array}{l}17.40 \\
5.82 \\
1.62\end{array}$ & $\begin{array}{l}7.28 \\
6.24 \\
1.26\end{array}$ & $\begin{array}{l}20.0 \\
5.77 \\
1.38\end{array}$ & & $\begin{array}{l}14.57 \\
5.32 \\
1.43\end{array}$ & $\begin{array}{l}3.33 \\
3.63 \\
2.69\end{array}$ & $\begin{array}{l}13.38 \\
4.94 \\
1.94\end{array}$ & $\begin{array}{l}4.10 \\
4.01 \\
1.80\end{array}$ & 1.90 & \begin{tabular}{|l|}
0.68 \\
1.88
\end{tabular} & 36 & 26 & & 0.13 & 0.99 & $\begin{array}{l}0.03 \\
0.26 \\
0.77\end{array}$ & \begin{tabular}{l|}
0.36 \\
0.18 \\
0.65
\end{tabular} & NA \\
\hline 41 & $\begin{array}{l}\text { Brain } \\
\text { Kidney } \\
\text { Liver } \\
\text { Muscle }\end{array}$ & $\begin{array}{l}\text { HTRA3 } \\
\text { HTRA3 } \\
\text { HTRA3 } \\
\text { HTRA3 }\end{array}$ & $\begin{array}{l}\text { STAG000000047613 } \\
\text { BTAG00000047613 } \\
\text { BTAG0000000047613 } \\
\text { BTAG00000047613 }\end{array}$ & $\begin{array}{c}7.62 \\
43.69\end{array}$ & $\begin{array}{c}9.71 \\
47.36\end{array}$ & 6.91 & $\begin{array}{c}7.05 \\
32.98\end{array}$ & 7.82 & 7.46 & 10.67 & 15.40 & $\begin{array}{l}11.60 \\
40.23\end{array}$ & 0.95 & 1.36 & 1.97 & 1.48 & 1.00 & 1.00 & 0.01 & 0.34 & NA \\
\hline 42 & $\begin{array}{l}\text { Brain } \\
\text { Kidney } \\
\text { Liver } \\
\text { Muscle }\end{array}$ & $\begin{array}{l}1 G F 2 \\
1 G F 2 \\
1 G F 2 \\
\text { IGF2 }\end{array}$ & $\begin{array}{l}\text { ENSBTAG000000013066 } \\
\text { ENSBTAG00000013066 } \\
\text { ENSBAGG00000013066 } \\
\text { ENSBTAG00000013066 }\end{array}$ & $\begin{array}{c}60.96 \\
9091.27 \\
4215.26 \\
9619.31\end{array}$ & $\begin{array}{c}76.95 \\
9586.41 \\
4920.90 \\
12219.01\end{array}$ & $\begin{array}{c}14.96 \\
10108.87 \\
4481.60 \\
8995.81\end{array}$ & $\begin{array}{c}31.37 \\
10866.42 \\
4657.53 \\
9115.27\end{array}$ & \begin{tabular}{|c|}
46.06 \\
9913.24 \\
4568.82 \\
9987.35
\end{tabular} & \begin{tabular}{|l}
10.60 \\
8757.28 \\
2805.21 \\
9970.52
\end{tabular} & $\begin{array}{c}40.54 \\
8392.55 \\
3969.01 \\
9411.92\end{array}$ & $\begin{array}{c}53.40 \\
11957.84 \\
5539.81 \\
12005.32\end{array}$ & $\begin{array}{c}288.67 \\
8343.93 \\
5585.75 \\
12735.20\end{array}$ & $\begin{array}{l}0.61 \\
1.00\end{array}$ & $\begin{array}{l}0.87 \\
0.94\end{array}$ & $\begin{array}{l}1.21 \\
1.20\end{array}$ & 1.28 & 0.08 & $\begin{array}{l}1.00 \\
1.00 \\
0.99 \\
1.00\end{array}$ & $\begin{array}{l}0.70 \\
0.47 \\
0.31 \\
0.41\end{array}$ & $\begin{array}{l}0.00 \\
0.45 \\
0.30 \\
0.26\end{array}$ & $\begin{array}{l}\text { Biallelic } \\
\text { Paternal } \\
\text { Paternal } \\
\text { Paternal }\end{array}$ \\
\hline 43 & $\begin{array}{l}\text { Brain } \\
\text { Kidney } \\
\text { Liver } \\
\text { Muscle }\end{array}$ & $\begin{array}{l}I G F 2 R \\
I G F 2 R \\
I G F 2 R \\
I G F 2 R\end{array}$ & $\begin{array}{l}\text { ENSBTAG000000002402 } \\
\text { ENSBTAG000000002402 } \\
\text { ENSBATAG000000002402 } \\
\text { ENSBTAG000000002402 }\end{array}$ & $\begin{array}{l}729.77 \\
473.96 \\
648.16\end{array}$ & $\begin{array}{l}765.07 \\
606.62 \\
868.58\end{array}$ & $\begin{array}{l}690.98 \\
486.72 \\
670.55\end{array}$ & $\begin{array}{l}652.22 \\
474.73 \\
650.52\end{array}$ & $\begin{array}{l}709.51 \\
510.51 \\
709.45\end{array}$ & $\begin{array}{c}438.47 \\
90.46 \\
269.10\end{array}$ & $\begin{array}{l}379.07 \\
272.28 \\
348.37\end{array}$ & $\begin{array}{l}307.08 \\
340.94\end{array}$ & $\begin{array}{l}404.97 \\
512.52\end{array}$ & $\begin{array}{l}0.18 \\
0.38\end{array}$ & $\begin{array}{l}0.53 \\
0.49\end{array}$ & $\begin{array}{l}0.60 \\
0.48\end{array}$ & $\begin{array}{l}0.79 \\
0.72\end{array}$ & 0.00 & $\begin{array}{l}0.00 \\
0.00\end{array}$ & $\begin{array}{l}0.00 \\
0.00 \\
0.00\end{array}$ & $\begin{array}{l}0.22 \\
0.00 \\
0.31 \\
0.11\end{array}$ & $\begin{array}{l}\text { Biallelic } \\
\text { Maternal } \\
\text { Maternal } \\
\text { Maternal }\end{array}$ \\
\hline 44 & $\begin{array}{l}\text { Brain } \\
\text { Kidney } \\
\text { Liver } \\
\text { Muscle }\end{array}$ & $\begin{array}{l}\text { IMPACT } \\
\text { IMPACT }\end{array}$ & $\begin{array}{l}\text { ENSBATGG0000000003035 } \\
\text { ENSBAGG00000003035 } \\
\text { ENSBTAG00000003035 }\end{array}$ & $\begin{array}{l}72.33 \\
33.51 \\
89.92\end{array}$ & $\begin{array}{l}32.94 \\
95.96\end{array}$ & $\begin{array}{r}29.16 \\
96.42\end{array}$ & $\begin{array}{l}29.92 \\
98.52\end{array}$ & $\begin{array}{l}31.38 \\
95.21\end{array}$ & $\begin{array}{l}30.49 \\
84.55\end{array}$ & $\begin{array}{l}28.35 \\
82.30\end{array}$ & $\begin{array}{l}23.73 \\
71.27\end{array}$ & $\begin{array}{l}26.01 \\
66.46\end{array}$ & 0.89 & 0.86 & 0.75 & 0.70 & 0.52 & $\begin{array}{l}1.00 \\
1.00\end{array}$ & $\begin{array}{l}0.17 \\
0.10\end{array}$ & $\begin{array}{l}0.88 \\
0.39 \\
0.01\end{array}$ & $\begin{array}{l}\text { Biallelic } \\
\text { Biallelec } \\
\text { Biallelic } \\
\text { Biallelelic }\end{array}$ \\
\hline 45 & Brain & INPP5F & ENSBTAG00000021801 & 37.85 & 315.16 & 734.90 & 806.53 & 3.6 & 69.37 & 454.27 & 627.67 & 534.65 & 0.70 & 0.67 & 0.93 & 0.79 & 0.24 & 0.38 & 0.76 & 0.64 & atemal \\
\hline
\end{tabular}




\begin{tabular}{|c|c|c|c|c|c|c|c|c|c|c|c|c|c|c|c|c|c|c|}
\hline Gene & ${ }^{7}$ Tissue & & $\begin{array}{l}\text { Weight } \rightarrow \\
\text { Fetus } \rightarrow \\
\text { Gene ID } \downarrow\end{array}$ & $\begin{array}{c}392 \mathrm{~g} \\
\text { Control \#1 }\end{array}$ & $\begin{array}{l}404 \mathrm{~g} \\
\text { Control \#2 } \\
\text { read cour }\end{array}$ & $\begin{array}{c}408 g \\
\text { Control \#3 } \\
\text { ts/million }\end{array}$ & $\begin{array}{c}416 \mathrm{~g} \\
\text { Control \#4 }\end{array}$ & Mean & $\begin{array}{c}514 \mathrm{~g} \\
\mathrm{LOS} \# 1\end{array}$ & $\begin{array}{c}518 \mathrm{~g} \\
\text { LoS \#2 } \\
\text { read cour }\end{array}$ & $\begin{array}{c}620 \mathrm{~g} \\
\text { Los \#3 } \\
\text { nts/million }\end{array}$ & $\begin{array}{c}714 \mathrm{~g} \\
\operatorname{LoS} \# 4\end{array}$ & Los \#1 & $\begin{array}{l}\text { Fold C } \\
\text { LoS \#2 }\end{array}$ & $\begin{array}{l}\text { hange } \\
\text { LoS \#3 }\end{array}$ & $\operatorname{Los} \# 4$ & $\begin{array}{r}\text { FDR } \\
\text { LOS \#1 }\end{array}$ & $\begin{array}{l}\text { FFalse DI } \\
\text { LOS \#2 }\end{array}$ \\
\hline & Kidney & $\begin{array}{l}\text { INPPSF } \\
\text { INPPSE }\end{array}$ & ENSBTAG000000 1801 & \begin{tabular}{|l|l|} 
& 64.64 \\
3 & 01 \\
\end{tabular} & $\begin{array}{l}63.75 \\
2854\end{array}$ & $\begin{array}{l}63.79 \\
3522\end{array}$ & $\begin{array}{l}54.91 \\
36.22\end{array}$ & $\begin{array}{l}61.77 \\
3397\end{array}$ & $\begin{array}{l}77.16 \\
43309\end{array}$ & 69.77 & $\begin{array}{l}48.71 \\
3978\end{array}$ & $\begin{array}{l}69.68 \\
38\end{array}$ & 1.25 & 1.13 & 0.79 & 1.13 & 0.63 & 1.00 \\
\hline & $\begin{array}{l}\text { Liver } \\
\text { Muscle }\end{array}$ & $\begin{array}{l}\text { INPPSF } \\
\text { INPPSF }\end{array}$ & $\begin{array}{l}\text { ENSBAAG000000021801 } \\
\text { ENSBTAG00000021801 }\end{array}$ & \begin{tabular}{|l|}
35.91 \\
120.81
\end{tabular} & $\begin{array}{l}28.54 \\
118.40\end{array}$ & $\begin{array}{l}35.22 \\
149.23\end{array}$ & $\begin{array}{c}36.22 \\
131.34\end{array}$ & $\begin{array}{c}33.97 \\
129.94\end{array}$ & $\begin{array}{r}43.09 \\
168.55\end{array}$ & $\begin{array}{l}40.84 \\
143.70\end{array}$ & $\begin{array}{l}39.78 \\
114.48\end{array}$ & $\begin{array}{l}38.98 \\
109.12\end{array}$ & $\begin{array}{l}1.27 \\
1.30\end{array}$ & $\begin{array}{l}1.20 \\
1.11\end{array}$ & $\begin{array}{l}1.17 \\
0.88\end{array}$ & $\begin{array}{l}1.15 \\
0.84\end{array}$ & $\begin{array}{l}0.19 \\
0.18\end{array}$ & $\begin{array}{l}0.91 \\
1.00\end{array}$ \\
\hline 46 & Brain & JADE1 & ENSBTAG 00000017493 & 40,42 & 54.53 & 41.96 & 3537 & 43.07 & & & & & & & & & & \\
\hline & Kidney & JADE1 & ENSBTAG00000017493 & 55.26 & 63.07 & 52.92 & 52.52 & 55.94 & $\begin{array}{l}94.24 \\
58.15\end{array}$ & $\begin{array}{l}55.43 \\
51.08\end{array}$ & $\begin{array}{l}81.67 \\
22.28\end{array}$ & 57.09 & 2.19 & 1.29 & 1.90 & 1.33 & 0.02 & 0.85 \\
\hline & Liver & JADE1 & ENSBTAG00000017493 & 27.65 & 28.77 & 23.98 & 24.81 & 26.31 & 15.11 & 28.26 & 30.80 & $\begin{array}{l}51.11 \\
26.69\end{array}$ & $\begin{array}{l}7.04 \\
0.57\end{array}$ & 1.07 & 1.17 & 1.01 & 0.00 & 0.99 \\
\hline & Muscle & JADE1 & ENSBTAG00000017493 & 50.96 & 76.00 & 52.81 & 59.77 & 59.88 & 32.25 & 52.50 & 64.10 & 62.52 & 0.54 & 0.88 & 1.07 & 1.04 & 0.00 & 1.00 \\
\hline 47 & Brain & KCNK9 & ENSBTAG000000047488 & 11.83 & 13.41 & 6.82 & 6.98 & 9.76 & 22.81 & 13.38 & 27.20 & 16.27 & 2.34 & 1.37 & 2.79 & 1.67 & 0.10 & 0.90 \\
\hline & $\begin{array}{c}\text { Kidney } \\
\text { Liver }\end{array}$ & $\begin{array}{l}\text { KCNKG } \\
\text { KCNK9 }\end{array}$ & ENSBTAG00000047488 & & & & & & & & & & & & & & & \\
\hline & Muscle & KCNK9 & ENSBTAG00000047488 & & & & & & & & & & & & & & & \\
\hline 48 & Brain & KCNQ1 & ENSBTAG00000010986 & 15.94 & 11.88 & 6.72 & 7.83 & 10.59 & 6.56 & 6.81 & 8.60 & 12.60 & 0.62 & 0.64 & 0.81 & 1.19 & 0.68 & 0.95 \\
\hline & Kidney & KCNQ1 & ENSBTAG00000010986 & $\begin{array}{l}21.32 \\
651\end{array}$ & 27.15 & 19.20 & 18.38 & $\begin{array}{r}21.51 \\
7.55\end{array}$ & $\begin{array}{l}23.75 \\
317\end{array}$ & 15.45 & 12.65 & 28.61 & 1.10 & 0.72 & 0.59 & 1.33 & 1.00 & 1.00 \\
\hline & Liver & KCNQ1 & ENSBTAG00000010986 & 6.51 & 14.09 & 3.92 & 5.69 & 7.55 & 3.17 & 5.34 & 8.28 & 8.13 & 0.42 & 0.71 & 1.10 & 1.08 & 0.15 & .00 \\
\hline & Muscle & KCNQ1 & ENSBTAG00000010986 & 34.24 & 47.85 & 27.10 & 35.12 & 36.08 & 16.35 & 23.01 & 34.70 & 69.03 & 0.45 & 0.64 & 0.96 & 1.91 & 0.00 & 0.59 \\
\hline 49 & Brain & KLF14 & 785436 & & & & & & & & & & & & & & & \\
\hline & Kidney & KLF14 & 785436 & 3.79 & 2.63 & 2.92 & 3.64 & 3.24 & 2.55 & 3.05 & 3.47 & 1.07 & 0.78 & 0.94 & 1.07 & 0.33 & 1.00 & 1.00 \\
\hline & Muscle & KLF14 & $\begin{array}{l}785436 \\
785436\end{array}$ & 5.76 & 4.51 & 2.79 & 4.60 & 4.42 & 5.22 & 4.19 & 4.29 & 2.86 & 1.18 & 0.95 & 0.97 & 0.65 & 0.73 & 1.00 \\
\hline 50 & Brain & L3MBTL1 & 529298 & 39.61 & 54.98 & 43.09 & 41.40 & 44.77 & 47.28 & 37.87 & 38.89 & 40.61 & 1.06 & 0.85 & 0.87 & 0.91 & 0.89 & 1.00 \\
\hline & Kidney & L3MBTL1 & 529298 & 13.28 & 16.60 & 16.42 & 16.39 & 15.67 & 16.67 & 12.62 & 12.47 & 16.70 & 1.06 & 0.81 & 0.80 & 1.07 & 1.00 & 1.00 \\
\hline & $\begin{array}{l}\text { Liver } \\
\text { Muscle }\end{array}$ & $\begin{array}{l}\text { L3MBBLT } \\
\text { LMBTL1 }\end{array}$ & $\begin{array}{l}529298 \\
529298\end{array}$ & $\begin{array}{l}6.14 \\
16.15\end{array}$ & $\begin{array}{l}8.87 \\
20.09\end{array}$ & $\begin{array}{l}7.61 \\
12.37\end{array}$ & $\begin{array}{l}7.37 \\
13.75\end{array}$ & $\begin{array}{l}7.49 \\
15.59\end{array}$ & $\begin{array}{l}2.20 \\
6.35\end{array}$ & $\begin{array}{c}5.42 \\
11.89\end{array}$ & $\begin{array}{l}5.79 \\
14.62\end{array}$ & $\begin{array}{c}7.91 \\
21.10\end{array}$ & $\begin{array}{l}0.29 \\
0.41\end{array}$ & $\begin{array}{l}0.72 \\
0.76\end{array}$ & $\begin{array}{l}0.77 \\
0.94\end{array}$ & $\begin{array}{l}1.06 \\
1.35\end{array}$ & $\begin{array}{l}0.00 \\
0.00\end{array}$ & $\begin{array}{l}0.94 \\
1.00\end{array}$ \\
\hline 51 & Brain & LIN28B & ENSBTAG00000043973 & 7.16 & 4.16 & 5.23 & 9.75 & 6.57 & 1.94 & 1.70 & 2.12 & 2.60 & 0.29 & 0.26 & 0.32 & 0.40 & 0.13 & 0.16 \\
\hline & $\begin{array}{c}\text { Kidney } \\
\text { Liver }\end{array}$ & $\begin{array}{l}{ }_{L}^{L} \text { LIN28B } \\
\text { LIN28B }\end{array}$ & $\begin{array}{l}\text { ENSBBAG000000043973 } \\
\text { ENSBTAG00000043973 }\end{array}$ & 32.61 & 22.49 & 27.11 & 29.34 & 27.89 & 26.83 & 25.37 & 19.04 & 23.44 & 0.96 & 0.91 & 0.68 & 0.84 & 0.58 & 0.92 \\
\hline & Muscle & LIN28B & ENSBTAG00000043973 & 年 & $22 .+0$ & T... & 20.07 & (7) & 20.00 & 年 & 0.07 & 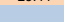 & 0.00 & 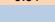 & 0.00 & 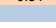 & 0.00 & 0.02 \\
\hline 52 & Brain & LRRTM1 & ENSBTAG000 & 24.89 & 33.47 & 27.15 & 33.80 & 29.83 & 49.43 & 28.93 & 28.46 & 30.38 & 1.66 & 0.97 & 0.95 & 1.02 & 0.29 & 1.00 \\
\hline & $\begin{array}{l}\text { Kidney } \\
\text { Liver }\end{array}$ & $\begin{array}{l}\text { LRRTM1 } \\
\text { LRRTM1 }\end{array}$ & $\begin{array}{l}\text { ENSBTAG00000002967 } \\
\text { ENSBTAG00000002967 }\end{array}$ & & & & & & & & & & & & & & & \\
\hline & Muscle & LRRTM1 & ENSBTAG000000002967 & & & & & & & & & & & & & & & \\
\hline 53 & Brain & MAGEL2 & ENSBTAG00000045998 & 34.24 & 29.90 & 31.87 & 45.23 & 35.31 & 23.56 & 19.46 & 27.08 & 42.20 & 0.67 & 0.55 & 0.77 & 1.20 & 0.43 & 38 \\
\hline & Kidney & MAGEL2 & 0000045998 & 19.94 & 22.02 & 16.19 & 18.35 & 19.12 & 16.29 & 23.98 & 28.32 & 32.27 & 0.85 & 1.25 & 1.48 & 1.69 & 1.00 & \\
\hline & Liver & MAGEL2 & ENSBTAG00000045998 & 8.97 & 9.34 & 5.67 & 9.08 & 8.26 & 5.24 & 7.12 & 13.10 & 12.69 & 0.63 & 0.86 & 1.5 & 1. & 0.23 & 0 \\
\hline & Muscle & MAGEL2 & ENSBTAG00000045998 & 17.66 & 20.85 & 15.12 & 17.13 & 17.69 & 24.67 & 18.02 & 24.19 & 34.37 & 1.39 & 1.02 & 1.37 & 1.94 & 0.04 & 1.00 \\
\hline 54 & Brain & MAG/2 & 100298336 & 51.52 & 47.46 & 42.54 & 46.32 & 46.96 & 117.37 & 67.19 & 67.33 & 59.42 & 2.50 & 1.43 & 1.43 & 1.27 & 0.01 & 82 \\
\hline & $\begin{array}{l}\text { Kidney } \\
\text { Liver }\end{array}$ & $M A G / 2$ & $\begin{array}{l}100298336 \\
100298336\end{array}$ & 20.63 & 21.89 & 20.08 & 19.50 & 20.52 & 20.44 & 17.21 & 4.28 & 16.84 & 1.00 & 0.84 & 0.21 & 0.82 & 1.00 & 1.00 \\
\hline & $\begin{array}{l}\text { Liver } \\
\text { Muscle }\end{array}$ & $\begin{array}{l}\text { MAGG/2 } \\
\text { MAG/2 }\end{array}$ & $\begin{array}{l}10002983336 \\
100298336\end{array}$ & & & & & & & & & & & & & & & \\
\hline 55 & Brain & MEST & ENSBTAG00000017223 & 118.33 & 14 & $10 s$ & 109.68 & 121.43 & 98.22 & 91.71 & 101 & 108.28 & 0.81 & 0.76 & 0.83 & 89 & 0.81 & 94 \\
\hline & Kidney & & ENSBTAG00000017223 & 213.51 & & 194.26 & 183.82 & 206.20 & 238.63 & 227.88 & 218.52 & 332.13 & 1.16 & 11 & 1.06 & 1 & 1.00 & 0 \\
\hline & Liver & MEST & ENSBTAG00000017223 & 22.11 & 20.05 & 20.46 & 19.35 & 20.49 & 31.51 & 18.12 & 19.48 & 42.14 & 1.54 & 0.88 & 0.95 & 2.06 & 0.00 & 96 \\
\hline & Muscle & MEST & ENSBTAG00000017223 & 1136.59 & 969.63 & 1173.48 & 1205.39 & 1121.27 & 1308.81 & 1385.65 & 931.59 & 1229.94 & 1.17 & 1.24 & 0.83 & 1.10 & 0.59 & 0.97 \\
\hline 56 & $\begin{array}{l}\text { Brain } \\
\text { Kidpey }\end{array}$ & MKRN3 & $\begin{array}{l}517464 \\
551744\end{array}$ & 14.31 & 10.46 & 17.68 & 16.59 & 14.76 & 14.46 & 12.53 & 16.69 & 14.64 & 1.01 & 1.20 & 0.94 & 0.88 & 0.90 & 0.97 \\
\hline & $\begin{array}{l}\text { Kidney } \\
\text { Liver }\end{array}$ & $\begin{array}{l}\text { MRRN3 } \\
\text { MKRN3 }\end{array}$ & $\begin{array}{l}517644 \\
517464\end{array}$ & & & & & & & & & & & & & & & \\
\hline & Muscle & MKRN3 & 517464 & & & & & & & & & & & & & & & \\
\hline 57 & Brain & MST1R & ENSBTAG00000015046 & 0.37 & 3.40 & 2.29 & 0.89 & 1.73 & 2.53 & 1.74 & 1.57 & 2.17 & 1.46 & 1.00 & 0.91 & 1.25 & 57 & 98 \\
\hline & Kidney & $\begin{array}{l}\text { MST1R } \\
\text { MSTR }\end{array}$ & ENSBTAG00000015046 & 3.75 & 2. & 3.6 & 3. & 3.51 & 3. & 1 & 5.22 & 5. & 1 & 0 & 1.50 & 1.44 & 1.00 & \\
\hline & $\begin{array}{l}\text { Liver } \\
\text { Muscle }\end{array}$ & $\begin{array}{l}\text { MSTIR } \\
\text { MST1R }\end{array}$ & $\begin{array}{l}\text { ENSBTAG00000015046 } \\
\text { ENSBTAG0000015046 }\end{array}$ & 1.43 & 2.20 & 1.75 & 1.23 & 1.65 & 0.48 & 1.32 & 1.73 & 1.43 & 0.29 & 0.80 & 1.05 & 0.86 & 0.02 & 1.00 \\
\hline 58 & Brain & NAAGO & ENSBTAG00000004875 & 66.16 & & 60.33 & 62.4 & 62.12 & 74 & & & 65.48 & 1.20 & 0.98 & 1.03 & 05 & 83 & 00 \\
\hline & Kidney & & 00000004875 & 29.09 & & & 31.81 & 29.35 & 29.79 & & & 32 & 1.02 & 1.08 & 1.37 & & & \\
\hline & Liver & NAAGO & ENSBTAG00000004875 & 19.87 & & 19 & 20 & 20.8 & 14.89 & 18.66 & 25 & 23. & 0.72 & 0.5 & 1.23 & 1 & 0.05 & \\
\hline & Muscle & NAAGO & ENSBTAG00000004875 & 34.48 & 31.60 & 26.58 & 29.35 & 30.50 & 29.74 & 36.24 & 43.03 & 38.39 & 0.98 & 1.19 & 1.41 & 1.2 & 0.97 & 0.95 \\
\hline 59 & Brain & NAP1L4 & 000022160 & 145.90 & 135.46 & 150.93 & 152.58 & 146.22 & 221.67 & 187.02 & 153.10 & 129.37 & 1.52 & 1.28 & 1.05 & 0.88 & 41 & 0.96 \\
\hline & Kidney & NAP1L4 & 00000022160 & 114.39 & 118.41 & 119.92 & 122.50 & 118.81 & 113.72 & 121.16 & & 36 & 0.96 & & 1.37 & & & \\
\hline & Liver & NAP1L4 & ENSBTAG00000022160 & 111.30 & 99.56 & 101.96 & 114.43 & 106.81 & 113.24 & 119.17 & 102.70 & 107.55 & 1.06 & 1.12 & 0.96 & 1.01 & 0.82 & 7 \\
\hline & Muscle & NAP1L4 & ENSBTAG00000022160 & 158.73 & 147.17 & 158.90 & 137.07 & 150.47 & 156.19 & 174.63 & 150.81 & 144.25 & 1.04 & 1.16 & 1.00 & 0.96 & 0.89 & 0.96 \\
\hline 60 & Brain & NAP1L5 & ENSB & 218.53 & 50.34 & 74.38 & 156.82 & 125.02 & 15.44 & 18.41 & 21.72 & 50.73 & 0.12 & 0.15 & 0.17 & 0.41 & 0.00 & 0.00 \\
\hline & Kidney & NAP1L5 & ENSB & 6.0 & & & 4. & 4.8 & 9 & & & & & & & & & \\
\hline & $\begin{array}{l}\text { Liver } \\
\text { Muscle }\end{array}$ & $\begin{array}{l}\text { NAP115 } \\
\text { NAP1L5 }\end{array}$ & ENSBTAG00000010128 & 20.65 & $\begin{array}{l}15.82 \\
456\end{array}$ & $\begin{array}{l}22.11 \\
6.93\end{array}$ & 19.84 & 19. & 35.25 & $\begin{array}{l}24.04 \\
887\end{array}$ & $\begin{array}{l}24.90 \\
5.22\end{array}$ & 21.2 & $12+2$ & $\begin{array}{l}1.23 \\
1.45\end{array}$ & 1.27 & 1.08 & & \\
\hline & muste & & ENSBTAG00000010128 & 0.19 & 4.00 & 0.90 & 6.80 & 6.12 & 1.00 & 8.81 & 5.22 & 6.05 & 1.25 & 1.45 & 0.85 & 0.99 & 0.71 & 0.94 \\
\hline 61 & $\mathrm{Br}$ & NL & ENSBTAG00000002186 & 然 & 57.93 & 96.34 & 86.01 & 76.5 & 87 & $6 \varepsilon$ & 71.69 & 79 & 1.15 & 0.90 & 0.94 & 1.04 & 0.97 & 0.97 \\
\hline & Kid & & & & & & & & & & & & & & & & & \\
\hline & Liver & NDN & ENSBT & 150 & & & & 13 & 12. & & & & & 0.9 & . & & & \\
\hline & Muscle & NDN & ENSBTAG00000002186 & 53.04 & 37.44 & 45.79 & 40.49 & 44.1 & 62.04 & 54.22 & 7.46 & 66.7 & 1.40 & 1.2 & 1.3 & 1.51 & 0.10 & 0.99 \\
\hline 62 & sic & & & & & 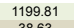 & 87.23 & 11 & & & & 99.53 & 0.8 & 0.6 & 0. & 0.82 & & 0.66 \\
\hline & Kic & & & & & & & & & & & & 1 & 1 & $?$ & & & \\
\hline & Liver & NNAT & ENSBTAG00000003212 & 2376.18 & 3377.11 & 2645.97 & 2339.23 & 2684.62 & 2627.99 & 2255.50 & 3887.36 & 2929.25 & 0.98 & 0.8 & 1.4 & 1. & 0.75 & (1) \\
\hline & Muscle & NNAT & ENSBTAG00000003212 & 77.83 & 69.00 & 81.74 & 49.69 & 69.57 & 101.78 & 79.34 & 169.78 & 167.76 & 1.46 & 1.14 & 2.44 & 2.41 & 0.09 & 1.00 \\
\hline 63 & $\begin{array}{c}\text { Brain } \\
\text { Kidney }\end{array}$ & $\begin{array}{l}\text { NTM } \\
\text { NTM }\end{array}$ & $\begin{array}{l}\text { ENSBTAG000000010032 } \\
\text { ENSBTAG00000010032 }\end{array}$ & $\begin{array}{c}206.53 \\
8.96\end{array}$ & $\begin{array}{c}121.64 \\
7.99\end{array}$ & $\begin{array}{c}197.17 \\
8.19\end{array}$ & $\begin{array}{c}273.62 \\
8.67\end{array}$ & $\begin{array}{l}199.74 \\
8.45\end{array}$ & $\begin{array}{l}67.88 \\
5.76\end{array}$ & $\begin{array}{c}74.15 \\
9.60\end{array}$ & $\begin{array}{l}106.20 \\
13.19\end{array}$ & $\begin{array}{l}97.39 \\
10.25\end{array}$ & $\begin{array}{l}0.34 \\
0.68\end{array}$ & $\begin{array}{l}0.37 \\
1.14\end{array}$ & $\begin{array}{l}0.53 \\
1.56\end{array}$ & $\begin{array}{l}0.49 \\
1.21\end{array}$ & $\begin{array}{l}0.00 \\
0.92\end{array}$ & $\begin{array}{l}0.01 \\
1.00\end{array}$ \\
\hline & Liver & NTM & ENSBTAG000000010032 & & & & & & & & & & & & & & & \\
\hline & Muscle & NTM & ENSBTAG00000010032 & 42.69 & 28.77 & 31.98 & 35.02 & 34.62 & 17.82 & 35.33 & 30.77 & 39.69 & 0.51 & 1.02 & 0.89 & 1.15 & 0.00 & 1.00 \\
\hline 64 & $\mathrm{Br}$ & 0 & EN & 39 & & & 42.8 & 36 & & & & & 1.23 & 0. & 1.00 & & & \\
\hline & Kid & & & & & & & & & & & & & & & & & \\
\hline & Liver & $8015-5+25$ & & 16 & & & 16 & & 15 & & & 19 & & 0. & 1. & & & \\
\hline & Muscle & OSBPL5 & ENSBTAG00000001077 & 16.72 & 22.35 & 15.08 & 16.16 & 17.58 & 18.61 & 18.12 & 22.74 & 27.41 & 1.06 & 1.03 & 1.29 & 1.56 & 0.50 & 1.00 \\
\hline 65 & $\mathrm{Br}$ & & & & & & & & & & & & & & & & & \\
\hline & & & & & & & & & & & & & & & & & & \\
\hline & Liver & PDE & ENSBT & 9. & 8. & & 19 & & & & & & 0. & 0.9 & 1.2 & 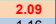 & & \\
\hline & Muscle & PDE10A & ENSBTAG00000007758 & 32.63 & 30.85 & 28.19 & 35.17 & 31.71 & 29.40 & 23.15 & 45.87 & 36.92 & 0.93 & 0.73 & 1.45 & 1.16 & 0.73 & 0.58 \\
\hline 66 & $\mathrm{Br}$ & $P L$ & EN & 10 & 117.3 & 15 & 185.36 & 140 & 205 & 193 & 15 & 10 & 1. 4 & 1. 5 & 1. & 0.76 & 0.60 & 0.94 \\
\hline & & & & & & & & 79 & & & & & 0. & 0. & 0. & & 0 & 1.0 \\
\hline & Liver & $P D E 4 D$ & ENSBTAG00000000 & 17 & 15 & 10 & 14. & 14 & 16.66 & 14. & 15 & & 1.13 & 0. & 1.08 & 0.96 & 0.59 & 1.00 \\
\hline & Muscle & $P D E 4 D$ & ENSBTAG00000000494 & 77.93 & 84.49 & 101.39 & 86.40 & 87.55 & 99.56 & 64.81 & 89.24 & 86.00 & 1.14 & 0.74 & 1.02 & 0.98 & 0.57 & 0.49 \\
\hline 67 & Brain & & & & & & & & & & & & 1.45 & 078 & 1.06 & & & \\
\hline & & PEG10 & ENSBTAG00000038093 & 547.19 & 548.67 & 442.65 & 405.93 & 486.11 & 446.98 & 380.80 & $50.1-5$ & & 0.92 & 0.78 & 0.10 & 0.94 & 1.00 & 1.00 \\
\hline
\end{tabular}




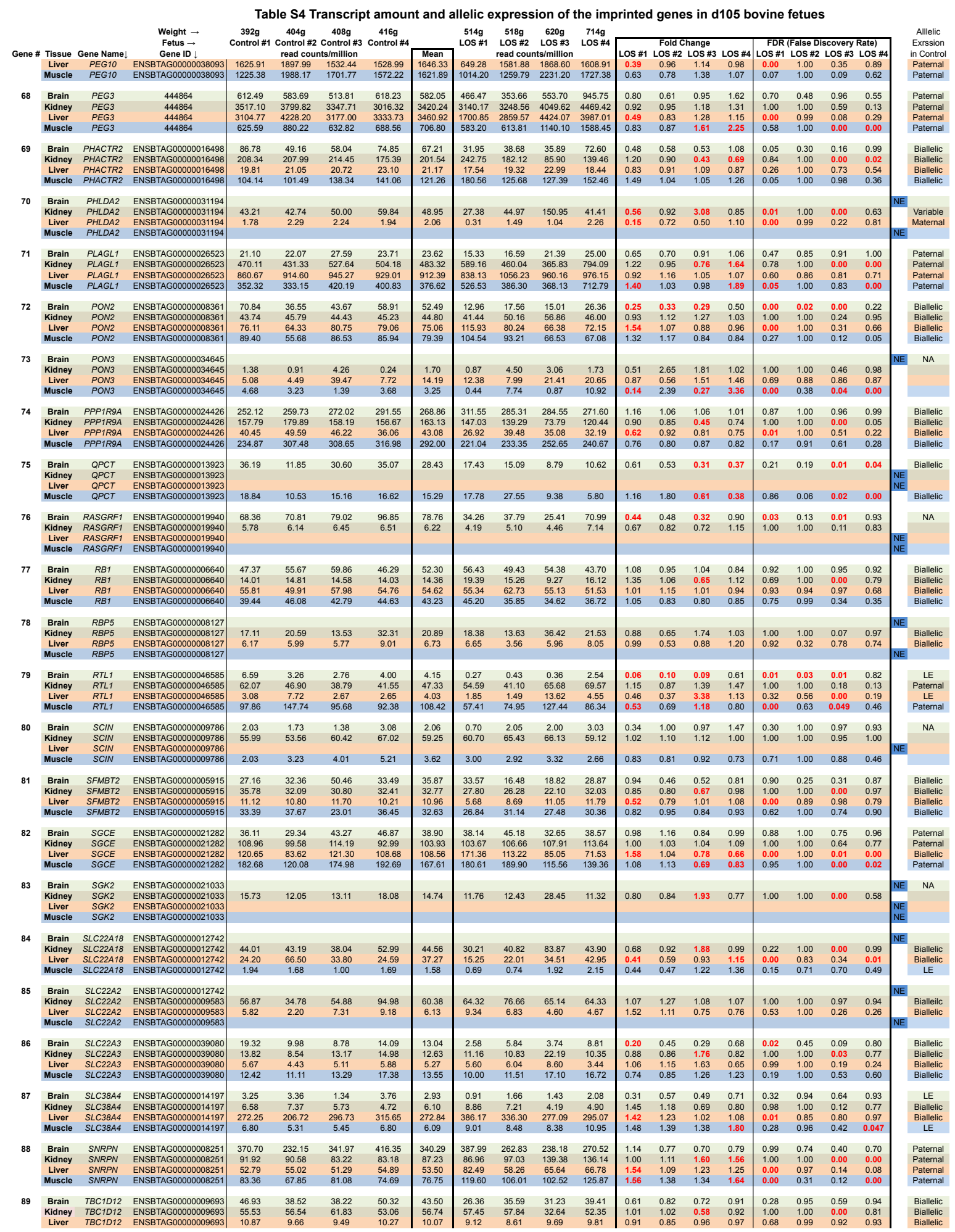




\begin{tabular}{|c|c|c|c|c|c|c|c|c|c|c|c|c|c|c|c|c|c|c|c|c|c|}
\hline \multirow[t]{2}{*}{ Gene \# } & \multirow{2}{*}{$\begin{array}{l}\text { Tissue } \\
\text { Muscle }\end{array}$} & \multirow{2}{*}{$\begin{array}{c}\text { Gene Name } \downarrow \\
T B C 1 D 12\end{array}$} & \multirow[t]{2}{*}{$\begin{array}{l}\text { Weight } \rightarrow \\
\text { Fetus } \rightarrow \\
\text { Gene ID } \downarrow \\
\text { ENSBTAG00000009693 }\end{array}$} & \multirow[t]{2}{*}{ 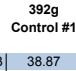 } & \multirow[t]{2}{*}{$\begin{array}{c}404 \mathrm{~g} \\
\text { Control \#2 } \\
\text { read cour } \\
38.29\end{array}$} & \multirow[t]{2}{*}{$\begin{array}{c}408 \mathrm{~g} \\
\text { Control \#3 } \\
\text { nts/million } \\
45.79\end{array}$} & $\begin{array}{c}416 \mathrm{~g} \\
\text { Control \#4 }\end{array}$ & \multirow{2}{*}{$\begin{array}{l}\text { Mean } \\
42.34\end{array}$} & $\begin{array}{r}514 \mathrm{~g} \\
\text { LoS \#1 }\end{array}$ & $\begin{array}{c}518 \mathrm{~g} \\
\text { LOS \#2 } \\
\text { read cour }\end{array}$ & $\begin{array}{c}620 \mathrm{~g} \\
\text { Los \#3 } \\
\text { its/million }\end{array}$ & $\begin{array}{c}714 \mathrm{~g} \\
\operatorname{LOS} \# 4\end{array}$ & LOS \#1 & $\begin{array}{l}\text { Fold } \\
\text { LoS \#2 }\end{array}$ & $\begin{array}{l}\text { hange } \\
\text { Los \#3 }\end{array}$ & & $\begin{array}{l}\text { FDR } \\
\operatorname{LOS} \# 1\end{array}$ & $\begin{array}{l}\text { (False D } \\
\text { LOS \#2 }\end{array}$ & iscovery & $\begin{array}{l}\text { Rate) } \\
\text { LOS \#4 }\end{array}$ & $\begin{array}{l}\text { Alllelic } \\
\text { Exrssion } \\
\text { in Contro }\end{array}$ \\
\hline & & & & & & & & & & & 37.86 & 48.72 & 1.29 & 1.03 & 0.89 & & 0.21 & & & 0.63 & Biallelic \\
\hline 90 & Brain & TCEB3 & ENSBTAG00000026585 & 80.72 & 112.35 & 87.08 & 69.59 & 87.43 & \begin{tabular}{|l|l|}
108.98 \\
\end{tabular} & 110.86 & 126.90 & 126.96 & 1.25 & 1.27 & 1.45 & 1.45 & 0.57 & 0.82 & 0.32 & 0.55 & Biallelic \\
\hline & Kidney & TCEB3 & ENSBTAG00000026585 & 129.20 & 132.90 & 120.05 & 110.36 & 123.13 & \begin{tabular}{|l}
125.65 \\
4583
\end{tabular} & 118.05 & 82.97 & $\begin{array}{l}123.00 \\
8525\end{array}$ & 1.02 & 0.96 & 0.67 & 1.00 & 1.00 & 1.00 & 0.00 & 1.00 & Biallelic \\
\hline & $\begin{array}{l}\text { Muscele } \\
\text { Muscla }\end{array}$ & $\begin{array}{l}\text { TCEB3 } \\
\text { TCEB3 }\end{array}$ & ENSBTAGO00000026558 & $\begin{array}{l}72.69 \\
86.81\end{array}$ & 115.21 & 93.81 & 91.87 & 96.92 & \begin{tabular}{|l}
45.83 \\
85.19
\end{tabular} & $\begin{array}{l}6.84 \\
90.92\end{array}$ & $\begin{array}{r}75.18 \\
110.45\end{array}$ & $\begin{array}{r}85.25 \\
133.59\end{array}$ & $\begin{array}{l}0.65 \\
0.88\end{array}$ & $\begin{array}{l}0.92 \\
0.94\end{array}$ & $\begin{array}{l}1.07 \\
1.14\end{array}$ & $\begin{array}{l}1.21 \\
1.38\end{array}$ & $\begin{array}{l}0.00 \\
0.75\end{array}$ & $\begin{array}{l}1.00 \\
1.00\end{array}$ & $\begin{array}{l}0.65 \\
0.21\end{array}$ & $\begin{array}{l}0.14 \\
0.00\end{array}$ & $\begin{array}{l}\text { Bailelelic } \\
\text { Biallelic }\end{array}$ \\
\hline 91 & Brain & TFPI2 & ENSBTAG00000015844 & & & & & & & & & & & & & & & & & & \\
\hline & Kidney & TFPI2 & ENSBTAG00000015844 & 46.38 & 49.66 & 30.34 & 33.93 & 40.08 & 31.64 & 58.10 & 36.92 & 26.26 & 0.79 & 1.45 & 0.92 & 0.66 & 1.00 & 0.69 & 0.36 & 0.20 & Biallelic \\
\hline & $\begin{array}{l}\text { Liver } \\
\text { Muscle }\end{array}$ & $\begin{array}{l}T F P 12 \\
\text { TFPI2 }\end{array}$ & $\begin{array}{l}\text { ENSBTAG000000015844 } \\
\text { ENSBTAG00000015844 }\end{array}$ & $\begin{array}{l}42.26 \\
31.17\end{array}$ & $\begin{array}{l}28.74 \\
24.74\end{array}$ & $\begin{array}{l}56.01 \\
2876\end{array}$ & $\begin{array}{l}47.59 \\
6.37\end{array}$ & $\begin{array}{l}43.65 \\
3676\end{array}$ & $\begin{array}{r}53.71 \\
4589\end{array}$ & $\begin{array}{l}42.66 \\
46.59\end{array}$ & $\begin{array}{l}34.36 \\
34.15\end{array}$ & $\begin{array}{l}26.07 \\
1901\end{array}$ & $\begin{array}{l}1.23 \\
1.25\end{array}$ & $\begin{array}{l}0.98 \\
1.27\end{array}$ & $\begin{array}{l}0.79 \\
0.93\end{array}$ & 60 & $\begin{array}{l}0.60 \\
0.77\end{array}$ & $\begin{array}{l}0.97 \\
100\end{array}$ & 0.07 & $\begin{array}{l}0.00 \\
0.01\end{array}$ & $\begin{array}{l}\text { Biallelic } \\
\text { Bialelici }\end{array}$ \\
\hline & Muscle & TFPI2 & ENSBTAG00000015844 & 31.17 & 24.74 & 28.76 & 62.37 & & 45.89 & 46.59 & 34.15 & 19.01 & 1.25 & 1.27 & 0.93 & 0.52 & 0.77 & 1.00 & 0.72 & 0.01 & Biallelic \\
\hline 92 & $\begin{array}{c}\text { Brain } \\
\text { Kidney }\end{array}$ & ${ }_{T H}^{T H}$ & $\begin{array}{l}\text { ENSBTAG00000026768 } \\
\text { ENSBTAG00000026768 }\end{array}$ & & & & & & & & & & & & & & & & & & $\mathrm{NA}$ \\
\hline & $\begin{array}{l}\text { Liver } \\
\text { Muscle }\end{array}$ & $\begin{array}{l}T H \\
T H\end{array}$ & $\begin{array}{l}\text { ENSBTAG00000026768 } \\
\text { ENSBTAG00000026768 }\end{array}$ & 1.03 & 3.11 & 1.58 & 3.17 & 2.22 & 1.28 & 3.19 & 2.15 & 1.62 & 0.58 & 1.43 & 0.97 & 0.73 & 0.51 & 0.88 & 0.91 & 0.85 & \\
\hline 93 & Brain & NFRSF 23 & ENSBTAG00000039129 & 7.16 & 6.44 & 3.88 & 2.67 & 5.04 & 3.01 & 3.95 & 5.22 & 5.05 & 0.60 & 0.78 & 1.04 & 1.00 & 0.76 & 1.00 & 0.94 & 0.99 & Biallelic \\
\hline & $\begin{array}{l}\text { Kindney } \\
\text { Liver }\end{array}$ & $\begin{array}{l}\text { TNFRFF23 } \\
\text { TNFRSF } 23\end{array}$ & $\begin{array}{l}\text { ENSBTAG000000039129 } \\
\text { ENSBAGG0000039129 }\end{array}$ & $\begin{array}{l}3.33 \\
5.95\end{array}$ & $\begin{array}{r}2.99 \\
11.27\end{array}$ & $\begin{array}{l}3.08 \\
7.87\end{array}$ & $\begin{array}{l}2.56 \\
0.90\end{array}$ & $\begin{array}{l}2.99 \\
6.50\end{array}$ & $\begin{array}{l}2.48 \\
1.63\end{array}$ & $\begin{array}{l}2.61 \\
1.74\end{array}$ & $\begin{array}{l}1.76 \\
2.65\end{array}$ & $\begin{array}{l}2.69 \\
2.12\end{array}$ & \begin{tabular}{|l}
0.83 \\
0.25
\end{tabular} & $\begin{array}{l}0.87 \\
0.27\end{array}$ & $\begin{array}{l}0.59 \\
0.41\end{array}$ & .33 & $\begin{array}{l}1.00 \\
0.14\end{array}$ & $\begin{array}{l}1.00 \\
0.68\end{array}$ & $\begin{array}{l}0.07 \\
0.55\end{array}$ & $\begin{array}{l}0.93 \\
0.35\end{array}$ & $\begin{array}{l}\text { Baillelic } \\
\text { Biallelic }\end{array}$ \\
\hline & Muscle & TNFRSF 23 & ENSBTAG00000039129 & 4.06 & 7.17 & 4.53 & 2.76 & 4.63 & 2.22 & 3.48 & 2.85 & 4.64 & 0.48 & 0.75 & 0.61 & 1.00 & 0.23 & 1.00 & 0.57 & 0.65 & Biallelic \\
\hline 94 & Brain & TP73 & ENSBTAG00000005812 & 2.52 & 6.69 & 5.52 & 1.40 & 4.03 & 4.20 & 6.54 & 3.60 & 14.09 & 1.04 & 1.62 & 0.89 & 3.49 & 0.86 & 0.81 & 0.95 & 0.12 & NA \\
\hline & $\begin{array}{l}\text { Kidney } \\
\text { Liver }\end{array}$ & $\begin{array}{l}\text { TP73 } \\
T \text { TP3 }\end{array}$ & $\begin{array}{l}\text { ENSBTAG000000005812 } \\
\text { ENSBTAG00000005812 }\end{array}$ & & & & & & & & & & & & & & & & & & \\
\hline & Muscle & TP73 & $\begin{array}{l}\text { ENSBTAG00000005812 } \\
\text {. }\end{array}$ & 4.86 & 8.68 & 3.96 & 4.60 & 5.53 & 6.01 & 4.96 & 2.37 & 1.50 & 1.09 & 0.90 & 0.43 & 0.27 & 0.42 & 1.00 & 0.10 & 0.00 & \\
\hline 95 & Brain & TRAPPC9 & ENSBTAG00000013955 & 53.60 & & 58.01 & 64.90 & 63.66 & & & & & 90 & 84 & 18 & .06 & 95 & 00 & 72 & .96 & Biallelic \\
\hline & Kidney & TRAPPC9 & ENSBTAG00000013955 & 28.78 & 29.33 & 27.82 & 32.14 & 29.52 & 25.78 & 27.66 & 33.36 & 29.54 & 87 & 94 & .13 & 00 & 1.00 & 1.00 & .89 & 1.00 & Biallelic \\
\hline & Liver & TRAPPC9 & ENSBTAG00000013955 & 15.85 & 28.95 & 18.61 & 16.90 & 20.08 & 13.00 & 15.72 & 27.12 & 25.06 & 0.65 & 0.78 & 1.35 & .25 & 0.10 & 1.00 & 0.00 & 0.01 & Biallelic \\
\hline & Muscle & TRAPPC9 & ENSBTAG00000013955 & 11.24 & 15.58 & 10.24 & 7.82 & 11.22 & 8.52 & 10.06 & 17.26 & 17.68 & 0.76 & 0.90 & 1.54 & 1.58 & 0.69 & 1.00 & 0.02 & 0.01 & Biallelic \\
\hline 96 & Brain & TSPAN32 & ENSBTAG00000002702 & 3.09 & 2.84 & 1.23 & 1.61 & 2.19 & 1.18 & 1.86 & 3.05 & 2.64 & 0.54 & 0.85 & 1.39 & 1.20 & 0.72 & 1.00 & 0.77 & 0.95 & $\mathrm{NA}$ \\
\hline & Liver & TSPAN32 & ENSBTAG00000002702 & 67.95 & 94.77 & 61.31 & 66.62 & 72.66 & 46.13 & 73.62 & 88.21 & 72.90 & 0.63 & 1.01 & 1.21 & 1.00 & 0.01 & 0.90 & 0.02 & 0.47 & \\
\hline & Muscle & TSPAN32 & ENSBTAG00000002702 & 1.46 & 1.77 & 1.26 & 2.05 & 1.64 & 0.69 & 1.30 & 1.95 & 1.58 & 0.42 & 0.80 & 1.19 & 0.97 & 0.11 & 1.00 & 0.70 & 0.98 & \\
\hline 97 & Brain & $T S S C 4$ & 47793 & 11. & 12.16 & 12.16 & 12.21 & 11. & 8.23 & 9.44 & 12.05 & 00 & 0.69 & 0.79 & 1.01 & 1.01 & 0.69 & 0.98 & 0.99 & 00 & Biallelic \\
\hline & Kidney & TSSC4 & 793 & 11.5 & 12 & 1 & 13. & 12. & 11 & 12 & 30 & $1442-24$ & & & & & & & & & Biallelic \\
\hline & Liver & $T S S C 4$ & & 17. & 2 & & 17.09 & 18 & & 14 & 22 & & & & & & & & & & iallelic \\
\hline & Muscle & TSSC4 & ENSBTAG00000047793 & 14.07 & 14.25 & 12.85 & 10.89 & 13.02 & 11.47 & 11.96 & 14.28 & 13.13 & 0.88 & 0.92 & 1.10 & 1.01 & 0.76 & 1.00 & 0.67 & 0.95 & Biallelic \\
\hline 98 & Brain & UBE $3 A$ & ENSBTA & 128.62 & 160.79 & 184.2 & 164.04 & 159.43 & 97 & 176.93 & 173.73 & 138.96 & 03 & 11 & 1.09 & 37 & 3 & 00 & 92 & 93 & Biallelic \\
\hline & Kidney & UBEЗA & 000002487 & 160.93 & 155.44 & 16 & & 15 & & & 108.09 & & & & & & & & & 72 & Biallelic \\
\hline & Liver & UBE3A & 000002487 & $\begin{array}{c}92.18 \\
1878\end{array}-2$ & 82.03 & & & 91.13 & 110.42 & 101.79 & $\begin{array}{r}87.15 \\
16567\end{array}$ & 10010 & & & & & 36 & 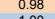 & 69 & 64 & Biallelic \\
\hline & Muscle & UBE3A & ENSBTAG00000002487 & 187.78 & 192.36 & 203.43 & 189.78 & 193.34 & 225.87 & 184.13 & 165.67 & 142.19 & 1.17 & 0.95 & 0.86 & 1.74 & 0.36 & 1.00 & 0.38 & 0.01 & Biallelic \\
\hline 99 & B & n & ENSBTAGO & & & & & & & & & & & & & & & & & & \\
\hline & $\begin{array}{l}\text { Kidney } \\
\text { Liver }\end{array}$ & $\begin{array}{l}\text { WIF1 } \\
\text { WF1 }\end{array}$ & $\begin{array}{l}\text { ENSBTAG00000014758 } \\
\text { ENSBTGG0000001458 }\end{array}$ & 10.26 & 8.96 & 10.06 & 11.84 & 10.28 & 10.53 & 13.25 & 18.50 & 14.70 & 1.02 & 1.25 & 1.80 & 1.43 & 1.00 & 1.00 & 0.01 & 0.30 & alelic \\
\hline & Muscle & WIF1 & ENSBTAG00000014758 & 0.94 & 1.02 & 0.39 & 0.77 & 0.78 & 2.02 & 1.13 & 1.58 & 4.39 & 2.59 & 1.44 & 2.03 & 5.62 & 0.02 & 0.96 & 0.12 & 0.00 & LE \\
\hline 100 & Kidno & WT & & & & & & & & & & & & & & & & & & & $\mathrm{NA}$ \\
\hline & Kidney & WT1 & & 92.27 & 90.22 & 110.52 & 108.20 & 100.30 & 113.23 & 95.80 & 108.68 & 99.63 & 1.13 & 0.96 & 1.08 & 0.99 & 1.00 & 1.00 & 0.89 & 1.00 & \\
\hline & $\begin{array}{l}\text { Liver } \\
\text { Muscle }\end{array}$ & WT1 & $\begin{array}{l}\text { ENSBATG000000047268 } \\
\text { ENSBAGG0000047268 }\end{array}$ & & & & & & & & & & & & & & & & & & \\
\hline 101 & Brain & & & & & & & & & & & & & & & & & & & & \\
\hline & Kidney & & & & & & & & & & & & & & & & & & & & iallelic \\
\hline & Liver & $\mathrm{ZC} 3 \mathrm{H} 12 \mathrm{C}$ & & 10.7 & & & & & & 11 & & & & & & & & & & & Biallelic \\
\hline & Muscle & $\mathrm{ZC} 3 \mathrm{H} 12 \mathrm{C}$ & ENSB & 36.41 & 36.0 & 41.04 & 43.76 & 39.3 & 43.8 & 33.50 & 35.20 & 34.03 & 1.12 & 0.85 & 0.90 & 0.87 & 0.70 & 0.95 & 0.58 & 0.29 & Biallelic \\
\hline 102 & Brain & $z$ & ENSBT & 9.23 & $15.1>2>1$ & 11.65 & 11.05 & 11.77 & 18.83 & 19.11 & $18 \mathrm{P}-2>3$ & 16.79 & 1.60 & 1.62 & 1.53 & 1.43 & 0.42 & 0.66 & 0.51 & 0.80 & Biallelic \\
\hline & Kid & & & & & & & & & & & & & & & & & & & & Biallelic \\
\hline & $\begin{array}{l}\text { Liver } \\
\text { Muscle }\end{array}$ & $\begin{array}{l}\text { ZFFAT } \\
\text { ZFAT }\end{array}$ & & 8.64 & $10.7-7>2>$ & 9.2 & $11.2>>2>$ & & & $\begin{array}{l}4.76 \\
10.03\end{array}$ & 12.01 & 14.03 & & 1.30 & & & 42 & & 46 & & Biallelic \\
\hline 103 & & & & & & & & & & & & & & & & & & & & & \\
\hline & Kid & & & & & & & & & & & & & & & & & & & & Biallelic \\
\hline & Liver & & & 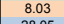 & & & & & & & & & & & & & & & 40 & & Biallelic \\
\hline & Muscle & ZFP64 & 300000001512 & 28.05 & 28.5 & 27 & 25 & 27. & 30.38 & 25.09 & 34.5 & 33. & 1.11 & 0.92 & 1.26 & 1.22 & 0.57 & 1.00 & 0.21 & 0.29 & Biallelic \\
\hline 104 & $\mathrm{Br}$ & & & & & & & & & & & & & & & & & 0.86 & 0.48 & 0.70 & NA \\
\hline & & & & & & & & & & & & & & & & & & & & & \\
\hline & & & ENSB & 4.7. & 6. & 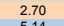 & 4 & 4 & & & & 2 & & & & & & & 管 & & \\
\hline & Muscle & ZNF215 & ENSBTAG00000003638 & 6.71 & 14.34 & 5.14 & 7.16 & 8.34 & 4.09 & 4.54 & 4.80 & 3.76 & 0.49 & 0.54 & 0.58 & 0.45 & 0.22 & 0.95 & 0.49 & 0.08 & \\
\hline 105 & $\mathrm{Bra}$ & & & & & & & 34. & & & 31. & & 1.40 & 1.29 & 0.91 & 0.86 & 0.64 & 0.97 & 31 & 0.91 & \\
\hline & & & & & & & & & & & & & & & & & & & & & Biallelic \\
\hline & & & & 5 & 5 & 5. & 5 & & & 6. & & & & & & & & & & & \\
\hline & Muscle & ZNF331 & ENSBTAG00000016513 & 12.28 & 11.07 & 12.42 & 14.52 & 12.57 & 14.08 & 13.23 & 10.04 & 10.75 & 1.12 & 1.05 & 0.80 & 0.86 & 0.80 & 1.00 & 0.37 & 0.39 & Biallelic \\
\hline
\end{tabular}


Table S5 Imprinted gene expression profile in each tissue of control fetuses

\begin{tabular}{|c|c|c|c|c|c|c|c|}
\hline \multirow[b]{2}{*}{ Gene Name $\downarrow$} & \multirow[b]{2}{*}{ Mean CPM } & \multirow[b]{2}{*}{ Min CPM } & \multirow[b]{2}{*}{ Max CPM } & \multicolumn{4}{|c|}{ CPM ratio to the average } \\
\hline & & & & Brain & Kidney & Liver & Muscle \\
\hline AIM1 & 26.27 & 10.35 & 58.91 & 0.63 & 2.11 & 0.73 & 0.54 \\
\hline AMPD3 & 9.06 & 4.42 & 15.79 & 1.48 & 0.52 & 0.00 & 0.00 \\
\hline ANO1 & 10.32 & 1.25 & 27.72 & 0.22 & 1.40 & 2.13 & 0.25 \\
\hline ART5 & 16.38 & 1.74 & 65.16 & 0.00 & 0.00 & 1.87 & 0.13 \\
\hline ASB4 & 13.73 & 0.82 & 35.95 & 0.00 & 0.00 & 0.12 & 1.88 \\
\hline ATP10A & 9.26 & 2.18 & 15.19 & 0.32 & 1.51 & 0.00 & 1.17 \\
\hline$A X L$ & 40.05 & 16.94 & 81.93 & 0.95 & 1.02 & 0.47 & 1.55 \\
\hline BEGAIN & 28.35 & 10.09 & 53.25 & 1.65 & 0.85 & 0.48 & 1.01 \\
\hline BLCAP & 175.00 & 59.01 & 362.57 & 1.55 & 0.34 & 0.82 & 1.29 \\
\hline$C A L C R$ & 18.11 & 8.49 & 39.69 & 1.02 & 0.00 & 0.00 & 0.98 \\
\hline CASD1 & 110.16 & 15.80 & 179.98 & 1.33 & 1.17 & 0.17 & 1.33 \\
\hline$C D 81$ & 162.45 & 120.49 & 227.68 & 1.05 & 0.93 & 0.86 & 1.16 \\
\hline CDKN1C & 90.09 & 8.24 & 252.95 & 0.17 & 1.03 & 0.77 & 2.02 \\
\hline CMAH & 19.00 & 1.45 & 39.89 & 0.00 & 0.10 & 1.90 & 0.00 \\
\hline CNTN3 & 26.01 & 6.41 & 53.32 & 0.99 & 0.30 & 0.00 & 1.71 \\
\hline COBL & 53.27 & 6.72 & 165.52 & 0.29 & 0.64 & 0.17 & 2.91 \\
\hline COMMD1 & 33.42 & 14.09 & 55.81 & 0.82 & 1.22 & 0.48 & 1.48 \\
\hline COPG2 & 29.44 & 9.51 & 56.79 & 1.61 & 0.65 & 0.33 & 1.42 \\
\hline CPA4 & 32.79 & 1.13 & 114.68 & 0.35 & 0.50 & 0.05 & 3.10 \\
\hline$D C N$ & 580.25 & 21.16 & 2079.08 & 0.09 & 0.84 & 0.13 & 2.95 \\
\hline$D D C$ & 12.61 & 5.77 & 20.23 & 0.00 & 0.56 & 1.44 & 0.00 \\
\hline DHCR7 & 66.42 & 19.53 & 135.74 & 1.58 & 0.59 & 1.42 & 0.41 \\
\hline DIO3 & 7.95 & 6.76 & 9.40 & 0.00 & 1.00 & 0.00 & 0.00 \\
\hline DIRAS3 & 271.25 & 15.38 & 688.40 & 1.92 & 0.08 & 0.00 & 0.00 \\
\hline DLGAP2 & 26.85 & 17.61 & 32.88 & 1.00 & 0.00 & 0.00 & 0.00 \\
\hline DLK1 & 685.60 & 4.61 & 1637.22 & 0.02 & 0.73 & 1.32 & 1.93 \\
\hline DLX5 & 6.17 & 0.94 & 13.07 & 1.00 & 0.00 & 0.00 & 0.00 \\
\hline DNMT1 & 91.20 & 67.66 & 125.51 & 0.87 & 1.06 & 1.25 & 0.82 \\
\hline DRD1A & 17.57 & 7.28 & 35.83 & 1.00 & 0.00 & 0.00 & 0.00 \\
\hline FBXO40 & 15.11 & 3.22 & 28.77 & 0.00 & 1.76 & 0.00 & 0.24 \\
\hline GATM & 408.71 & 11.15 & 1228.00 & 0.30 & 0.92 & 2.74 & 0.04 \\
\hline GDAP1L1 & 61.31 & 53.31 & 64.79 & 1.00 & 0.00 & 0.00 & 0.00 \\
\hline GLIS3 & 6.97 & 1.70 & 14.39 & 0.90 & 1.73 & 0.00 & 0.37 \\
\hline GNAS & 2024.94 & 1038.77 & 3009.67 & 0.92 & 1.20 & 0.53 & 1.35 \\
\hline GPR1 & 0.69 & 0.49 & 0.86 & 1.00 & 0.00 & 0.00 & 0.00 \\
\hline GRB10 & 32.43 & 18.26 & 42.72 & 0.72 & 0.90 & 1.23 & 1.15 \\
\hline GTL2 & 4365.28 & 1587.00 & 12651.22 & 0.68 & 0.64 & 0.45 & 2.23 \\
\hline$H 13$ & 45.13 & 28.97 & 65.23 & 0.78 & 1.32 & 1.07 & 0.84 \\
\hline H19 & 7243.57 & 18.75 & 16180.77 & 0.01 & 1.93 & 0.84 & 1.23 \\
\hline HTR2A & 7.11 & 1.26 & 20.00 & 2.05 & 0.75 & 0.20 & 0.00 \\
\hline HTRA3 & 22.53 & 6.91 & 47.36 & 0.00 & 0.35 & 0.00 & 1.65 \\
\hline IGF2 & 6128.87 & 14.96 & 12219.01 & 0.01 & 1.62 & 0.75 & 1.63 \\
\hline IGF2R & 495.40 & 37.50 & 868.58 & 0.11 & 1.43 & 1.03 & 1.43 \\
\hline IMPACT & 68.55 & 29.16 & 98.52 & 1.11 & 1.05 & 0.46 & 1.39 \\
\hline INPP5F & 224.82 & 28.54 & 837.85 & 3.00 & 0.27 & 0.15 & 0.58 \\
\hline JADE1 & 46.30 & 23.98 & 76.00 & 0.93 & 1.21 & 0.57 & 1.29 \\
\hline KCNK9 & 9.76 & 6.82 & 13.41 & 1.00 & 0.00 & 0.00 & 0.00 \\
\hline KCNQ1 & 18.93 & 3.92 & 47.85 & 0.56 & 1.14 & 0.40 & 1.91 \\
\hline KLF14 & 3.83 & 2.63 & 5.76 & 0.00 & 0.85 & 0.00 & 1.15 \\
\hline L3MBTL1 & 20.88 & 6.14 & 54.98 & 2.14 & 0.75 & 0.36 & 0.75 \\
\hline LIN28B & 17.23 & 4.16 & 32.61 & 0.38 & 0.00 & 1.62 & 0.00 \\
\hline LRRTM1 & 29.83 & 24.89 & 33.80 & 1.00 & 0.00 & 0.00 & 0.00 \\
\hline MAGEL2 & 20.10 & 5.67 & 45.23 & 1.76 & 0.95 & 0.41 & 0.88 \\
\hline MAG/2 & 33.74 & 19.50 & 51.52 & 1.39 & 0.61 & 0.00 & 0.00 \\
\hline MEST & 367.35 & 19.35 & 1205.39 & 0.33 & 0.56 & 0.06 & 3.05 \\
\hline MKRN3 & 14.76 & 10.46 & 17.68 & 1.00 & 0.00 & 0.00 & 0.00 \\
\hline MST1R & 2.30 & 0.37 & 3.95 & 0.75 & 1.53 & 0.72 & 0.00 \\
\hline NAA60 & 35.69 & 19.87 & 66.16 & 1.74 & 0.82 & 0.58 & 0.85 \\
\hline NAP1L4 & 130.58 & 99.56 & 158.90 & 1.12 & 0.91 & 0.82 & 1.15 \\
\hline NAP1L5 & 38.92 & 4.01 & 218.53 & 3.21 & 0.13 & 0.50 & 0.16 \\
\hline NDN & 44.19 & 12.27 & 96.34 & 1.73 & 0.96 & 0.31 & 1.00 \\
\hline NNAT & 975.64 & 33.09 & 3377.11 & 1.14 & 0.04 & 2.75 & 0.07 \\
\hline NTM & 80.94 & 7.99 & 273.62 & 2.47 & 0.10 & 0.00 & 0.43 \\
\hline OSBPL5 & 27.82 & 14.41 & 45.03 & 1.32 & 1.42 & 0.62 & 0.63 \\
\hline PDE10A & 46.49 & 8.37 & 204.76 & 2.49 & 0.55 & 0.28 & 0.68 \\
\hline PDE4D & 80.33 & 10.74 & 185.36 & 1.74 & 0.98 & 0.18 & 1.09 \\
\hline PEG10 & 1128.54 & 405.93 & 1988.17 & 0.67 & 0.43 & 1.46 & 1.44 \\
\hline PEG3 & 2042.50 & 513.81 & 4228.20 & 0.28 & 1.67 & 1.69 & 0.35 \\
\hline PHACTR2 & 102.79 & 19.81 & 214.45 & 0.65 & 1.96 & 0.21 & 1.18 \\
\hline PHLDA2 & 25.50 & 1.78 & 59.84 & 0.00 & 1.92 & 0.08 & 0.00 \\
\hline PLAGL1 & 448.99 & 21.10 & 945.27 & 0.05 & 1.08 & 2.03 & 0.84 \\
\hline PON2 & 62.94 & 36.55 & 89.40 & 0.83 & 0.71 & 1.19 & 1.26 \\
\hline PON3 & 6.38 & 0.24 & 39.47 & 0.00 & 0.27 & 2.23 & 0.51 \\
\hline PPP1R9A & 191.77 & 36.06 & 316.98 & 1.40 & 0.85 & 0.22 & 1.52 \\
\hline QPCT & 21.86 & 10.53 & 36.19 & 1.30 & 0.00 & 0.00 & 0.70 \\
\hline RASGRF1 & 42.49 & 5.78 & 96.85 & 1.85 & 0.15 & 0.00 & 0.00 \\
\hline RB1 & 41.13 & 14.01 & 59.86 & 1.27 & 0.35 & 1.33 & 1.05 \\
\hline RBP5 & 13.81 & 5.77 & 32.31 & 0.00 & 1.51 & 0.49 & 0.00 \\
\hline RTL1 & 40.98 & 2.65 & 147.74 & 0.10 & 1.15 & 0.10 & 2.65 \\
\hline
\end{tabular}

CPM: counts per million Min: mimum CPM in tissues of control fetuses

CPM ratio: the average CPM of each tissue was divded by Mean CPM Boxed $=$ not expressed 
Table S5 Imprinted gene expression profile in each tissue of control fetuses

\begin{tabular}{|c|c|c|c|c|c|c|c|}
\hline \multirow[b]{2}{*}{ Gene Name $\downarrow$} & \multirow[b]{2}{*}{ Mean CPM } & \multirow[b]{2}{*}{ Min CPM } & \multirow[b]{2}{*}{ Max CPM } & \multicolumn{4}{|c|}{ CPM ratio to the average } \\
\hline & & & & Brain & Kidney & Liver & Muscle \\
\hline SCIN & 21.64 & 1.38 & 67.02 & 0.10 & 2.74 & 0.00 & 0.17 \\
\hline SFMBT2 & 28.06 & 10.21 & 50.46 & 1.28 & 1.17 & 0.39 & 1.16 \\
\hline SGCE & 104.75 & 29.34 & 192.69 & 0.37 & 0.99 & 1.04 & 1.60 \\
\hline SGK2 & 14.74 & 12.05 & 18.08 & 0.00 & 1.00 & 0.00 & 0.00 \\
\hline SLC22A18 & 27.80 & 1.00 & 66.50 & 0.00 & 1.60 & 1.34 & 0.06 \\
\hline$S L C 22 A 2$ & 33.25 & 2.20 & 94.98 & 0.00 & 1.82 & 0.18 & 0.00 \\
\hline$S L C 22 A 3$ & 11.12 & 4.43 & 19.32 & 1.17 & 1.14 & 0.47 & 1.22 \\
\hline$S L C 38 A 4$ & 71.99 & 1.34 & 315.65 & 0.04 & 0.08 & 3.79 & 0.08 \\
\hline SNRPN & 139.44 & 51.29 & 416.35 & 2.44 & 0.63 & 0.38 & 0.55 \\
\hline TBC1D12 & 38.16 & 9.49 & 61.83 & 1.14 & 1.49 & 0.26 & 1.11 \\
\hline TCEB3 & 94.55 & 67.80 & 132.90 & 0.92 & 1.30 & 0.75 & 1.03 \\
\hline TFPI2 & 40.16 & 24.74 & 62.37 & 0.00 & 1.00 & 1.09 & 0.92 \\
\hline $\mathrm{TH}$ & 2.22 & 1.03 & 3.17 & 0.00 & 0.00 & 1.00 & 0.00 \\
\hline TNFRSF23 & 4.79 & 0.90 & 11.27 & 1.05 & 0.62 & 1.36 & 0.97 \\
\hline TP73 & 4.78 & 1.40 & 8.68 & 0.84 & 0.00 & 0.00 & 1.16 \\
\hline TRAPPC 9 & 31.12 & 7.82 & 78.12 & 2.05 & 0.95 & 0.65 & 0.36 \\
\hline TSPAN32 & 25.50 & 1.23 & 94.77 & 0.09 & 0.00 & 2.85 & 0.06 \\
\hline TSSC4 & 13.97 & 10.89 & 22.49 & 0.85 & 0.88 & 1.34 & 0.93 \\
\hline UBE3A & 150.29 & 82.03 & 203.43 & 1.06 & 1.05 & 0.61 & 1.29 \\
\hline WIF1 & 5.53 & 0.39 & 11.84 & 0.00 & 1.86 & 0.00 & 0.14 \\
\hline$W T 1$ & 100.30 & 90.22 & 110.52 & 0.00 & 1.00 & 0.00 & 0.00 \\
\hline $\mathrm{ZC} 3 \mathrm{H} 12 \mathrm{C}$ & 34.46 & 10.74 & 51.11 & 1.35 & 1.18 & 0.32 & 1.14 \\
\hline ZFAT & 9.91 & 3.33 & 15.95 & 1.19 & 1.44 & 0.37 & 1.00 \\
\hline ZFP64 & 26.01 & 7.35 & 39.15 & 1.33 & 1.31 & 0.32 & 1.05 \\
\hline ZNF215 & 9.73 & 2.70 & 20.96 & 1.50 & 1.15 & 0.49 & 0.86 \\
\hline ZNF331 & 20.97 & 5.01 & 39.42 & 1.63 & 1.51 & 0.26 & 0.60 \\
\hline
\end{tabular}


Table S6. Validation of SNPs identified by RNAseq using Sanger sequencing

\begin{tabular}{|c|c|c|c|c|c|c|c|c|c|}
\hline \multirow[b]{2}{*}{ Gene symbol } & \multirow[b]{2}{*}{ Chr } & \multirow[b]{2}{*}{ Position } & \multirow[b]{2}{*}{$\begin{array}{l}\text { Reference } \\
\text { (UMD3.1) }\end{array}$} & \multicolumn{3}{|c|}{ Sanger Sequencing of genomic DNA } & \multicolumn{2}{|c|}{ RNAseq (F1 hybrid fetuses) } & \multirow[b]{2}{*}{ SNP flanking Sequence } \\
\hline & & & & $\begin{array}{c}\text { B.t. } \\
\text { taurus }\end{array}$ & $\begin{array}{c}\text { B.t. } \\
\text { indicus }\end{array}$ & $\begin{array}{c}\text { F1 hybrid } \\
\text { fetuses }\end{array}$ & $\begin{array}{c}\text { Reference } \\
\text { allele }\end{array}$ & $\begin{array}{l}\text { Alternative } \\
\text { allele }\end{array}$ & \\
\hline MEST $T^{*}$ & 4 & 95073135 & A & A & A & A/A(LOS \#4) & A & G & \\
\hline SGCE & 4 & 11856585 & c & C & $\mathrm{T}$ & NA & C & $\mathrm{T}$ & atcaggttga $(\mathrm{C} / \mathrm{T})$ agaacaaagc \\
\hline PEG10 & 4 & 11921764 & $\mathrm{~T}$ & $\mathrm{~T}$ & c & NA & $\mathrm{T}$ & c & caccttgacc $(\mathrm{T} / \mathrm{C})$ ggaacttcag \\
\hline PEG10 & 4 & 11921800 & $\mathrm{~T}$ & $\mathrm{~T}$ & C & NA & $\mathrm{T}$ & c & gcattgttca(T/C)tggctggaag \\
\hline PEG10 & 4 & 11921824 & G & G & A & NA & G & A & gccacctgaa $(\mathrm{G} / \mathrm{A})$ tcttgggagc \\
\hline PEG10 & 4 & 11922094 & $\mathrm{~T}$ & $\mathrm{~T}$ & c & NA & $\mathrm{T}$ & c & $\operatorname{ctgacttcct}(\mathrm{T} / \mathrm{C})$ tagcctggcc \\
\hline PEG10 & 4 & 11922259 & G & G & C & NA & G & c & ctgggagcat(G/C)tgttcaaca \\
\hline PEG10 & 4 & 11922533 & A & A & C & NA & A & c & tcttaaatca(A/C)ttaaaattga \\
\hline NAP1L5 & 6 & 37510035 & c & C & $\mathrm{T}$ & NA & C & $\mathrm{T}$ & taaaatacta $(\mathrm{C} / \mathrm{T})$ gaaattgacg \\
\hline NAP1L5 & 6 & 37510055 & c & c & $\mathrm{T}$ & NA & c & $\mathrm{T}$ & gcaatcagat $(\mathrm{C} / \mathrm{T})$ atgcccagcg \\
\hline NAP1L5 & 6 & 37510166 & A & A & G & NA & A & G & tgtgaggact( $(\mathrm{A} / \mathrm{G})$ gagttgagac \\
\hline NAP1L5 & 6 & 37510180 & C & C & A & NA & C & A & ttgagactta(C/A)taagggtgtc \\
\hline NAP1L5 & 6 & 37510241 & A & A & G & NA & A & G & tcgtcacagt( $(\mathrm{A} / \mathrm{G})$ tcgcactagg \\
\hline NAP1L5 & 6 & 37510307 & G & G & C & NA & G & c & tcttccattt(G/C)ttttgcattt \\
\hline NAP1L5 & 6 & 37510534 & C & C & $\mathrm{T}$ & N/A & C & $\mathrm{T}$ & atgacctgta $(\mathrm{C} / \mathrm{T})$ aatattgtc \\
\hline NAP1L5 & 6 & 37510546 & $\mathrm{~T}$ & $\mathrm{~T}$ & C & NA & $\mathrm{T}$ & c & atattgtcaa $(\mathrm{T} / \mathrm{C})$ gtgtgtgttc \\
\hline NNAT & 13 & 67120130 & A & $\mathrm{A} / \mathrm{T}$ & $\mathrm{A} / \mathrm{T}$ & $\mathrm{A} / \mathrm{T}(\operatorname{LOS} \# 4)$ & A & $\mathrm{T}$ & cagcaccgac(A/T)atgacgacaa \\
\hline NNAT & 13 & 67120164 & C & $\mathrm{C} / \mathrm{T}$ & $\mathrm{C} / \mathrm{T}$ & $\mathrm{C} / \mathrm{T}(\operatorname{LOS} \# 4)$ & C & $\mathrm{T}$ & $\operatorname{ccccacccct}(\mathrm{C} / \mathrm{T}) \operatorname{tgcacccctc}$ \\
\hline NNAT & 13 & 67120187 & G & $\mathrm{G} / \mathrm{T}$ & $\mathrm{G} / \mathrm{T}$ & G/T(LOS \#4) & G & $\mathrm{T}$ & actgcgggtg $(\mathrm{G} / \mathrm{T})$ cctagaggga \\
\hline NNAT & 13 & 67120502 & G & $\mathrm{G} / \mathrm{A}$ & $\mathrm{G} / \mathrm{A}$ & G/A(LOS \#4) & G & A & ctttgcctac(G/A)gcatgtgcat \\
\hline NNAT & 13 & 67120662 & c & $\mathrm{C} / \mathrm{T}$ & $\mathrm{C} / \mathrm{T}$ & C/T(LOS \#4) & C & $\mathrm{T}$ & ccctcactga $(\mathrm{C} / \mathrm{T})$ cttgaatccc \\
\hline PEG3 & 18 & 64275301 & C & $\mathrm{T}$ & C & NA & C & $\mathrm{T}$ & agaagaacat $(\mathrm{C} / \mathrm{T})$ gagcgcaaga \\
\hline PEG3 & 18 & 64275316 & c & C & G & NA & C & G & aggcacgtgc $(\mathrm{C} / \mathrm{G})$ aagaagaaga \\
\hline PEG3 & 18 & 64275553 & A & A & G & NA & A & G & gcctcaggga(A/G)gacggggagcc \\
\hline PEG3 & 18 & 64275646 & $\mathrm{~T}$ & $\mathrm{~T}$ & C & NA & $\mathrm{T}$ & C & gccccgaaga $(\mathrm{T} / \mathrm{C})$ ggccaggagg \\
\hline PEG3 & 18 & 64275685 & C & C & $\mathrm{C} / \mathrm{T}$ & NA & C & $\mathrm{T}$ & aggatgagga $(\mathrm{C} / \mathrm{T})$ gaagagaagg \\
\hline PEG3 & 18 & 64275732 & G & G & $\mathrm{G} / \mathrm{A}$ & NA & G & A & gtccatgccc $(\mathrm{G} / \mathrm{A})$ gctctcagaa \\
\hline PEG3 & 18 & 64281735 & $\mathrm{~T}$ & $\mathrm{~T}$ & $\mathrm{C} / \mathrm{T}$ & $\mathrm{C} / \mathrm{T}(\mathrm{LOS} \# 3, \# 4$, and Control \#4) & $\mathrm{T}$ & c & aggcgatgta( $(\mathrm{T} / \mathrm{C})$ gagccggaag \\
\hline PEG3 & 18 & 64281750 & $\mathrm{~T}$ & $\mathrm{~T}$ & $\mathrm{C} / \mathrm{T}$ & $\mathrm{C} / \mathrm{T}(\mathrm{LOS} \# 3, \# 4) ; \mathrm{T} / \mathrm{T}($ Control \#4) & $\mathrm{T}$ & c & tcctggaaga $(\mathrm{T} / \mathrm{C})$ tacgaggcga \\
\hline MAGEL2 & 21 & 783853 & A & A & c & $\mathrm{A} / \mathrm{C}(\mathrm{LOS} \# 4)$ & A & c & aggtgcaaac $(A / C)$ cccatccaat \\
\hline MAGEL2 & 21 & 784015 & c & C & $\mathrm{T}$ & C/T(LOS \#4) & C & $\mathrm{T}$ & gggccctggg $(\mathrm{C} / \mathrm{T}) \operatorname{ctctgggaaa}$ \\
\hline MAGEL2 & 21 & 784070 & C & C & $\mathrm{T}$ & C/T(LOS \#4) & C & $\mathrm{T}$ & gctccctact(C/T)ttgctcaggg \\
\hline MAGEL2 & 21 & 784354 & $\mathrm{~T}$ & $\mathrm{~T}$ & C & T/C(LOS \#4) & $\mathrm{T}$ & c & tcaacaaggg $(\mathrm{T} / \mathrm{C})$ agaaagggtg \\
\hline MAGEL2 & 21 & 784789 & $\mathrm{~T}$ & $\mathrm{~T}$ & c & T/C(LOS\#3) & $\mathrm{T}$ & c & ataaaaccga $(\mathrm{T} / \mathrm{C})$ gaccccacca \\
\hline SNRPN & 21 & 8725 & A & A & G & NA & A & G & agcttgcatt(A/G)tttgtaagag \\
\hline SNRPN & 21 & 19911 & A & A & G & NA & A & G & gcctcactga(A/G)caaccaggag \\
\hline$S N R P N^{*}$ & 21 & 25855 & c & c & c & $\mathrm{C} / \mathrm{C}(\operatorname{LOS} \# 4)$ & c & A & \\
\hline
\end{tabular}

Two SNPs (indicated by asterisk) were not confirmed by Sanger seq
syndrome; NA: not analyzed; SNP: single nucleotide polymorphism;

Table S7. Identification of SNPs at DMRs by Sanger sequencing

\begin{tabular}{|c|c|c|c|c|c|c|}
\hline DMRs & Chr & Position & B.t.taurus & B. t. indicus & F1 hybrid fetus & SNP flanking sequence \\
\hline PLAGL1 DMR & 9 & 82473774 & G & $\mathrm{T}$ & G/T(LOS \#4, Control \#4 ) & $\operatorname{acgggcggac}(\mathrm{G} / \mathrm{T})$ gggcggctct \\
\hline SNRPN DMR & 21 & 26268 & A & G & A/G(LOS \#4, Control \#2, \#4) & ttaacaaaaa $(\mathrm{A} / \mathrm{G})$ gaaataccca \\
\hline NNAT DMR & 13 & 67118693 & G & $\mathrm{A} / \mathrm{G}$ & $\begin{array}{c}\mathrm{G} / \mathrm{G}(\text { Control \#4), G/A } \\
\text { (Control \#2, LOS \#3, \#4) }\end{array}$ & ttctggggac $(\mathrm{G} / \mathrm{A})$ cgcatcggga \\
\hline
\end{tabular}

DMR: differentially methylated region; Chr: Chromosome; The chromosome positions are based on UMD3.1 reference assembly. 
Table S8. PCR primer information

\begin{tabular}{|c|c|c|c|c|c|c|}
\hline Genes & Amplicon locations & Primer types & Primer sequences $\left(5^{\prime}-3^{\prime}\right)$ & Annealing temperature & Amplicon size & Assays \\
\hline MEST & Chr 4: 95072724-95073233 & Forward & CTAGATGGTACAGCGTTGAG & $59.1^{\circ} \mathrm{C}$ & 510bp & gDNA-PCR for SNP validation \\
\hline \multirow{2}{*}{ SGCE } & Chr 4. 11856803-11856067 & Reverse & CGTCACTGCCGTTTCTAA & & & \\
\hline & Chr 4: 11856803-11856067 & $\begin{array}{l}\text { Forward } \\
\text { Reverse }\end{array}$ & $\begin{array}{l}\text { GGACCTAGAAAGGCCAATATAA } \\
\text { GTTCCACAGTCTCTGCTTAC }\end{array}$ & $59.1^{\circ} \mathrm{C}$ & 737bp & gDNA-PCR for SNP validation \\
\hline \multirow[t]{2}{*}{ PEG10 } & Chr 4: 11921664-11922608 & Forward & CAGTCATGTATGAGGGCTAATG & $62.3^{\circ} \mathrm{C}$ & 945bp & gDNA-PCR for SNP validation \\
\hline & & Reverse & GCCTCTTATTCACAGTGTTGTA & & & \\
\hline \multirow[t]{2}{*}{ NAP1L5 } & Chr 6: $36808477-36809288$ & Forward & CTGCCGCCAAAGATGAG & $59.1^{\circ} \mathrm{C}$ & 812bp & gDNA-PCR for SNP validation \\
\hline & & Reverse & GGGATTTAATGCTTAAACAATTGGG & & & \\
\hline \multirow[t]{2}{*}{ NNAT } & Chr 13: 67119624-67120409 & Forward & CCATTGCGAGAAGTGAGGTAT & $58.4^{\circ} \mathrm{C}$ & 804bp & gDNA-PCR for SNP validation \\
\hline & & Reverse & ACACAGGCACAGAGTTGTC & & & \\
\hline \multirow[t]{2}{*}{ NNAT } & Chr 13: 67120338-67120931 & Forward & TACACCAGCCAGCAGAATG & $58.4^{\circ} \mathrm{C}$ & $615 \mathrm{bp}$ & gDNA-PCR for SNP validation \\
\hline & & Reverse & ACCCAGACCAGTCTTAGAAGTA & & & \\
\hline \multirow[t]{2}{*}{ PEG3 } & Chr 18: 64275728-64275179 & Forward & CTCAGAAGAGTCACACGATCAC & $59.8^{\circ} \mathrm{C}$ & $550 \mathrm{bp}$ & gDNA-PCR for SNP validation \\
\hline & & Reverse & GAGCTACGGACAAAGAACTCTC & & & \\
\hline \multirow[t]{2}{*}{ PEG3 } & Chr 18: 64276162-64275271 & Forward & CCTGATTGAGCACCAGAAGAT & $59.8^{\circ} \mathrm{C}$ & 892bp & gDNA-PCR for SNP validation \\
\hline & & Reverse & CATCATGGAGGCGTCGTAAT & & & \\
\hline \multirow[t]{2}{*}{ PEG3 } & Chr 18: 64282227-64281459 & Forward & GGGACATGTGTGTCTCCTTAC & $59.8^{\circ} \mathrm{C}$ & $770 \mathrm{bp}$ & gDNA-PCR for SNP validation \\
\hline & & Reverse & GCTGCTGTTGTCGTCTGA & & & \\
\hline \multirow[t]{2}{*}{ MAGEL2 } & Chr 21: 783758-784573 & Forward & GAGGAGGATAGTAGTGGCAAGA & $58.4^{\circ} \mathrm{C}$ & $816 \mathrm{bp}$ & gDNA-PCR for SNP validation \\
\hline & & Reverse & CTCCAGGTACTTCTCCCTTACA & & & \\
\hline \multirow[t]{2}{*}{ MAGEL2 } & Chr 21: 784158-784946 & Forward & TGCAGTTCCTCTTGGTTAAGG & $58.4^{\circ} \mathrm{C}$ & 789bp & gDNA-PCR for SNP validation \\
\hline & & Reverse & GAAATCCAAACTTACACACACAGA & & & \\
\hline \multirow[t]{2}{*}{ SNRPN } & Chr 21: 8930-8548 & Forward & TGGGAGATAAGGTCATCAAATAG & $59.1^{\circ} \mathrm{C}$ & 383bp & gDNA-PCR for SNP validation \\
\hline & & Reverse & CACCTCACACTCTGGAAATC & & & \\
\hline \multirow[t]{2}{*}{ SNRPN } & Chr 21: 20218-19677 & Forward & GGGCTAGATTCTGACCATTG & $59.1^{\circ} \mathrm{C}$ & $542 \mathrm{bp}$ & gDNA-PCR for SNP validation \\
\hline & & Reverse & GCTGAAGGAGGTAGAACTTTAT & & & \\
\hline \multirow[t]{2}{*}{ SNRPN } & Chr 21: 26236-25797 & Forward & ACCACTCTGGGTTGAAGTA & $59.1^{\circ} \mathrm{C}$ & 440bp & gDNA-PCR for SNP validation \\
\hline & & Reverse & CTGGAAGCTCAGGATGATTAAA & & & \\
\hline \multirow[t]{2}{*}{ PLAGL1 } & Chr 9: 82419707-82420135 & Forward & CTGCGACAGATGCTTCTAC & $60.7^{\circ} \mathrm{C}$ & 429bp & RT-PCR for allelic expression \\
\hline & & Reverse & GCGCTGTACTTGTGATTCT & & & \\
\hline \multirow[t]{2}{*}{ PEG3 } & Chr 18: 64280579-64281817 & Forward & GGATCAAGCCTTTGGGTGTA & $64.7^{\circ} \mathrm{C}$ & $299 \mathrm{bp}$ & RT-PCR for allelic expression \\
\hline & Spanning 2 introns & Reverse & GTCCCGTGACTCCATGTC & & & \\
\hline \multirow[t]{2}{*}{ SNRPN } & Chr 21: 8676-19987 & Forward & CACTTTAGACGGACTACAGAAC & $63.7^{\circ} \mathrm{C}$ & 302bp & RT-PCR for allelic expression \\
\hline & Spanning 2 introns & Reverse & ACCTAAGGGAAGGCTACAGATA & & & \\
\hline \multirow[t]{2}{*}{ NNAT } & Chr 13: 67120319-67120605 & Forward & CCACAGAGATCCAGACAACTAC & $63.1^{\circ} \mathrm{C}$ & $287 \mathrm{bp}$ & RT-PCR for allelic expression \\
\hline & & Reverse & CGTGCTCAGTAGGTCCAAAT & & & \\
\hline \multirow[t]{2}{*}{ PALGL1 DMR } & Chr 9: 82473614-82473911 & Forward & GTTGAGGGYGGTAAAAGTTTA & $60.5^{\circ} \mathrm{C}$ & 298bp & DNA methylation assay \\
\hline & & Reverse & CRACTACAAACCTCCCCAC & & & \\
\hline \multirow[t]{2}{*}{ SNRPN DMR } & Chr 21: 25757-26322 & Forward & TGGAAAGTTTGAGGAAATTTGA & $60.5^{\circ} \mathrm{C}$ Touchdown PCR & $566 \mathrm{bp}$ & DNA methylation assay \\
\hline & & Reverse & AAAACCCAAATCCCCAATAAAT & See SI methods & & \\
\hline NNAT DMR & Chr 13: 67118612-67118953 & Forward & ATTTGGTGTAGTAGGATTTTTTTAT & $60.5^{\circ} \mathrm{C}$ Touchdown PCR & $342 \mathrm{bp}$ & DNA methylation assay \\
\hline
\end{tabular}




\section{Chapter 3 Global misregulation of genes largely uncoupled to DNA methylome epimutations characterizes a congenital overgrowth syndrome}

\subsection{Abstract}

Assisted reproductive therapies (ART) have become increasingly common worldwide and numerous retrospective studies have indicated that ART-conceived children are more likely to develop the overgrowth syndrome Beckwith-Wiedemann (BWS). In bovine, the use of ART can induce a similar overgrowth condition, which is referred to as large offspring syndrome (LOS). Both BWS and LOS involve misregulation of imprinted genes. However, it remains unknown whether molecular alterations at non-imprinted loci contribute to these syndromes. Here we examined the transcriptome of skeletal muscle, liver, kidney, and brain of control and LOS bovine fetuses and found that different tissues within LOS fetuses have perturbations of distinct gene pathways. Notably, in skeletal muscle, multiple pathways involved in myoblast proliferation and fusion into myotubes are misregulated in LOS fetuses. Further, characterization of the DNA methylome of skeletal muscle demonstrates numerous local methylation differences between LOS and controls; however, only a small percent of differentially expressed genes (DEGs), including the imprinted gene $I G F 2 R$, could be associated with the neighboring differentially methylated regions. In summary, we not only show that misregulation of non-imprinted genes and loss-of-imprinting characterize the ART-induced overgrowth syndrome but also demonstrate that most of the DEGs is not directly associated with DNA methylome epimutations. 


\subsection{Introduction}

The use of ART has become increasingly common worldwide and each year approximately six percent of infants born in developed countries are conceived employing these technologies (European et al., 2016). The use of ART involves manipulation of gametes and/or embryos in an artificial environment, and it has been reported that ART-conceived children have increased risk for birth defects

(Grafodatskaya et al., 2013). For example, a meta-analysis of eight epidemiologic studies indicates that ART-conceived children have a 5.2-fold increased likelihood of developing the congenital overgrowth condition BWS (Vermeiden and Bernardus, 2013). However, it is still unresolved whether the use of ART or the infertility per se is the main cause of the loss-of-imprinting disorders observed in ART-conceived humans(Vermeiden and Bernardus, 2013). BWS is characterized by complex and variable symptoms including pre- and post-natal overgrowth, enlarged tongue, ear malformation, umbilical hernia, and predisposition to develop childhood tumors (Weksberg et al., 2010). In ruminants, gametes and embryos subjected to in vitro manipulations can develop into unusually large offspring that share phenotypes with BWS (Chen et al., 2013; Young et al., 1998). LOS, as the congenital overgrowth syndrome is referred to in ruminants, can cause detrimental effects to both the dam and offspring such as delivery difficulty due to the oversized fetus and inability for the newborn to stand and to suckle (Young et al., 1998). It is not clear what triggers the development of these congenital overgrowth conditions and associated phenotypes and why ART potentiates the syndromes. Numerous animal studies have indicated that the use of ART can alter the epigenome of the gametes and embryos (de Waal et al., 2012a; de Waal et al., 2012b; Market-Velker et al., 2010; Rivera 
et al., 2008) and this can contribute to the etiology of the ART-induced overgrowth conditions (Kuentz et al., 2011; Vermeiden and Bernardus, 2013).

DNA methylation is an epigenetic modification involving the addition of a methyl group to the $5^{\text {th }}$ carbon of cytosine (Smith and Meissner, 2013). In mammals, DNA methylation typically occurs in a $\mathrm{CpG}$ context with the exception of $\mathrm{CpH}$ methylation, which is mostly observed in neural tissues, oocytes and embryonic stem cells (He and Ecker, 2015; Shirane et al., 2013). DNA methylation plays a key role in many biological processes such as regulation of tissue-specific gene expression, suppression of parasite DNA in the genome, X-chromosome inactivation, and genomic imprinting (Smith and Meissner, 2013). Genomic imprinting is an epigenetic phenomenon in which a subset of genes, known as imprinted genes, are transcribed monoallelically in a parental-origindependent manner (Bartolomei and Ferguson-Smith, 2011). The transcriptional asymmetry of the parental alleles is usually directed by allele-specific DNA methylation (ASM) at imprinted loci established during gametogenesis (Bartolomei and FergusonSmith, 2011). Proper allele-specific DNA methylation and allele-specific expression of imprinted genes play important roles in the regulation of embryonic and neonatal growth, placental function, postnatal behavior, and metabolism (Plasschaert and Bartolomei, 2014). Altered gene expression and DNA methylation at imprinted loci have been associated with congenital overgrowth disorders such as BWS in human (Kalish et al., 2014) and LOS in bovine (Chen et al., 2015; Chen et al., 2013).

Most BWS cases are sporadic and have been linked to two imprinted loci on chromosome 11p15.5, the KCNQ1 locus and the H19/IGF2 locus (Weksberg et al., 2010). Approximately $50 \%$ of the BWS cases are associated with the hypomethylation of 
KvDMR1 at the KCNQ1 locus and 2-7\% are linked to the hypermethylation of the differentially methylated region (DMR) at the H19/IGF2 locus (Weksberg et al., 2010). In addition, studies have shown that a subset of BWS patients with epimutation at the $K C N Q 1$ locus also exhibit abnormal DNA methylation at other imprinted loci (Eggermann et al., 2016). We have observed loss of methylation of the KvDMR1 on the maternal allele in LOS (Chen et al., 2013) and have reported that LOS is a multi-locus loss-of-imprinting condition in which aberrant imprinted gene expression is associated with tissue-specific loss of imprinted DNA methylation (Chen et al., 2015).

Although it is well accepted that loss-of-imprinting can contribute to these overgrowth syndromes, and as such have been coined "loss-of-imprinting syndromes", it remains unknown whether aberrant gene expression and DNA methylation occur at nonimprinted loci and to what extent these molecular alterations contribute to the variable phenotypes observed in these conditions. To address this question, we examined the transcriptome of skeletal muscle, liver, kidney, and brain of four control and four LOS day $\sim 105$ Bos taurus indicus (B. t. indicus) $\times$ Bos taurus taurus (B. t.taurus) $\mathrm{F}_{1}$ fetuses (Chen et al., 2015). We found that different LOS fetuses exhibit different numbers of DEGs and that each tissue within LOS fetuses have perturbations of distinct gene pathways. Notably, in skeletal muscle, multiple pathways involved in myoblast proliferation and fusion into myotubes are misregulated in LOS fetuses. Further, characterization of the DNA methylome of skeletal muscle revealed numerous local methylation differences between LOS and controls; however, very few DEGs could be linked to neighboring identified DMRs. This study indicates that global misregulation of non-imprinted genes in addition to loss-of-imprinting characterizes the ART-induced 
overgrowth syndrome. The observation that most identified DMRs could not be directly associated with aberrant gene expression suggests that caution should be exercised when making conclusions about the etiology of such syndromes by interpreting time-pointspecific DNA methylation data.

\subsection{Results}

\section{Identification and characterization of DEGs in LOS fetuses}

In order to determine to what extent the transcriptome is altered in bovine LOS fetuses, we analyzed RNA sequencing (RNAseq) data of skeletal muscle, liver, kidney, and brain from four control and four LOS day $\sim 105(\mathrm{~d} 104-106)$ B. t. indicus $\times$ B. t. taurus $F_{1}$ fetuses that we generated in a previous study (Chen et al., 2015) (Table 1). These tissues were selected because they represent the three primary germ layers (i.e., liver for endoderm, kidney and skeletal muscle for mesoderm, and brain for ectoderm). Only females were used in this study to avoid any potential sex-specific gene expression (Ingleby et al., 2014b). The average bodyweight for control and LOS fetuses was 405g $[$ standard deviation $(\mathrm{SD})=10 \mathrm{~g}$, range $=24 \mathrm{~g}]$ and $592 \mathrm{~g}(\mathrm{SD}=95 \mathrm{~g}$, range $=200 \mathrm{~g})$, respectively $(\mathrm{p}=0.008)$ (Table 1$)$. The bodyweight differences between the control and LOS fetuses are not expected to be due to paternal effects because all fetuses were sired by one Nelore bull [ABS CSS MR N OB 425/1 677344 29NE0001 97155 (i.e., B. t. indicus)](Chen et al., 2013).

As LOS fetuses exhibited dramatic difference in bodyweight, each LOS fetus was considered individually and was compared to the mean of the four controls to identify 
DEGs. To ensure the validity of grouping four control fetuses for DEG identification, principle component analyses (PCA) were performed using the normalized RNAseq read counts. For RNAseq the expression libraries of kidney, skeletal muscle, and liver, control fetuses clustered while the libraries from the LOS fetuses did not (Fig. S1). It should be noted that the RNAseq libraries of skeletal muscle and liver from Control \#2 fetus were sequenced with 50bp read length; while the RNAseq reads of other fetuses were of $100 \mathrm{bp}$. The read length differences may explain why Control \#2 is segregated from other control fetuses in these two tissue types. In brain, both control and LOS fetuses exhibited a segregated pattern in the PCA plot (Fig. S1). In order to minimize the false positive DEGs caused by natural biological variation and technical variation, the DEGs were identified with the consideration of variance among the controls and the RNAseq read length differences using the edgeR package as we previously described (Anders et al., 2013; Chen et al., 2015; Robinson et al., 2010). The number of DEGs varied between tissues for different LOS fetuses (Fig. S2). For example, in the largest LOS fetus analyzed (weight $=714 \mathrm{~g}$ ), the number of DEGs was 3868, 1581, 441, and 276 for skeletal muscle, liver, kidney, and brain, while in the smallest LOS fetus (weight $=$ 514g), the number of DEGs for those tissues were 2270, 3232, 139, and 1106 (Fig. S2). The number of DEGs was not associated with fetal weight, but the fetuses with loss-ofimprinting at the KCNQ1 locus (i.e., LOS \#1 and \#4) had more DEGs in liver and skeletal muscle than LOS fetuses with correct imprinting at this locus (Fig. S2). Next, we performed Kyoto Encyclopedia of Genes and Genomes (KEGG) pathway analyses (Huang da et al., 2009) to gain biological insights of the DEGs identified in the LOS fetuses. We found that several KEGG pathways were shared by DEGs in different 
A

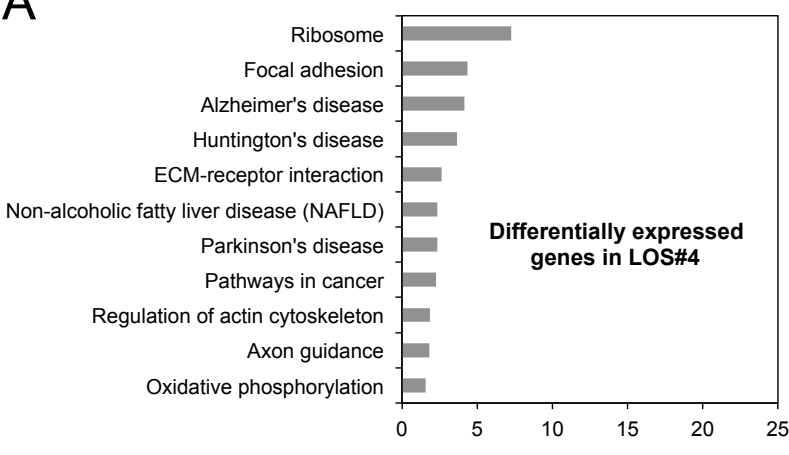

B

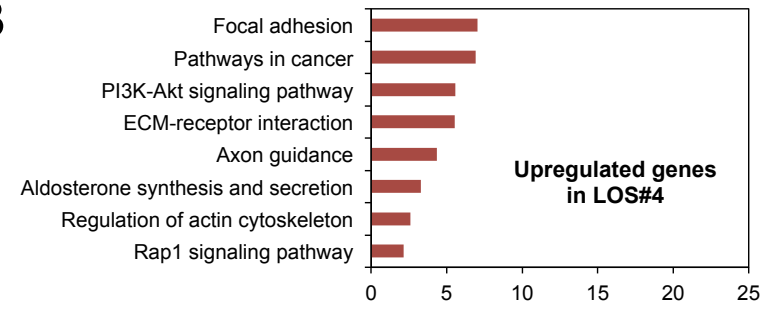

Ribosome Oxidative phosphorylation Parkinson's disease Alzheimer's disease Huntington's disease Non-alcoholic fatty liver disease (NAFLD) Cardiac muscle contraction Metabolic pathways

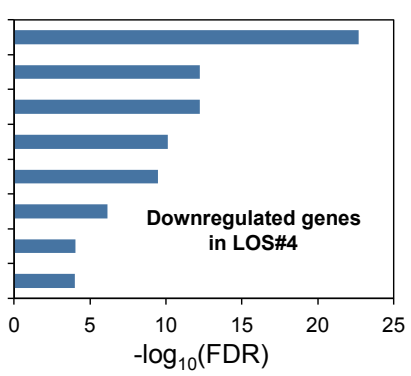

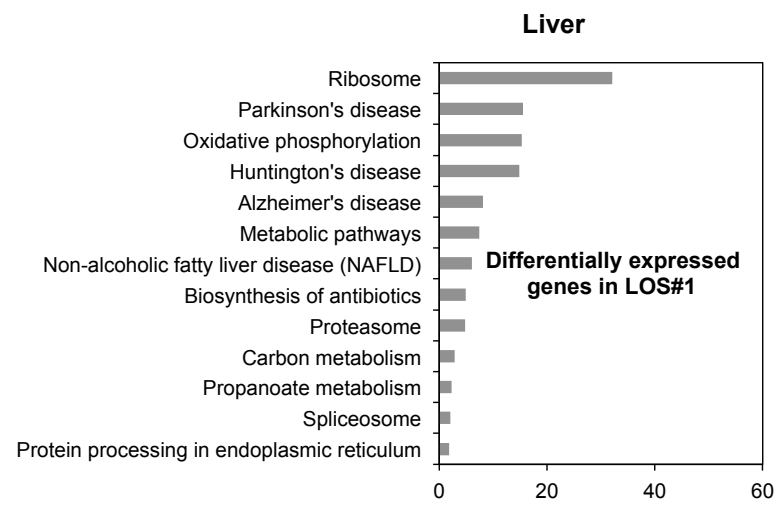

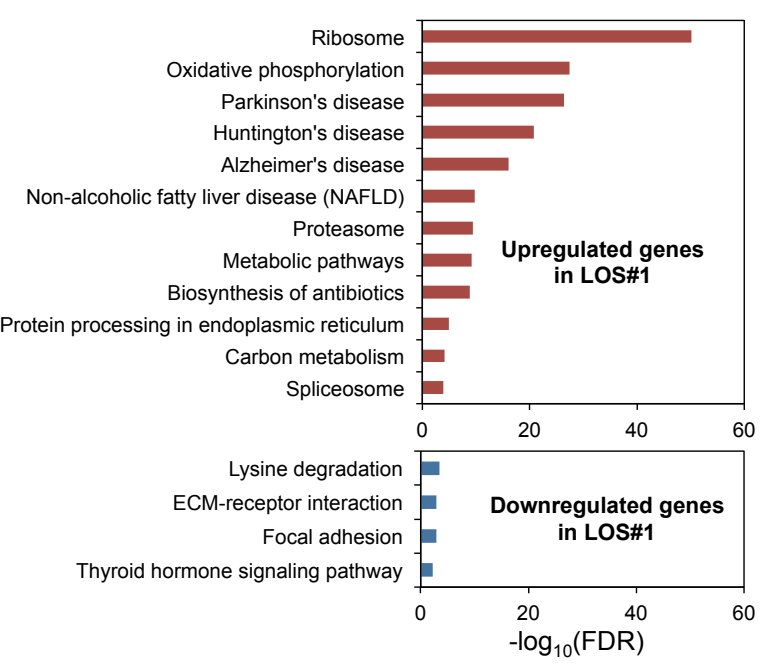

Figure 1. KEGG pathway analyses of DEGs in somatic tissues of LOS fetuses. A). KEGG pathways enriched for DEGs in skeletal muscle and liver (FDR <0.05). Shown here are examples of tissues with the most DEGs. B). KEGG pathways enriched for upregulated and downregulated genes in skeletal muscle and liver (FDR < 0.05). DEG: differentially expressed genes; LOS: large offspring syndrome. 
tissues while others were unique for a particular tissue (Fig. 1A and Fig. S2). For example, "ribosome" and "oxidative phosphorylation" were enriched in DEGs in skeletal muscle, liver, and kidney, while "pathways in cancer" was unique for skeletal muscle (Fig. 1A and Fig. S2), suggesting the over-proliferation of the muscle cells in LOS fetuses. Further analyses revealed that even the common pathways could be differentially represented in different tissues. For instance, "ribosome" and "oxidative phosphorylation" were enriched with downregulated genes in skeletal muscle but enriched with upregulated genes in liver and kidney (Fig. 1B and Fig. S2). For brain, however, misregulated genes were only found to enrich two KEGG pathways (Fig. S2). Overall, we found that numbers of DEGs varied among LOS fetuses and different tissues within LOS fetuses have perturbations of distinct gene pathways.

\section{Multiple pathways involved in myoblast proliferation and fusion into myotubes} are misregulated in LOS

To identify DEGs correlated with the overgrowth phenotype of the LOS fetuses, weighted gene correlation network analyses (WGCNA) (Langfelder and Horvath, 2008) were used to identify clusters of genes that were highly interconnected, namely modules, and to determine which modules were correlated with bodyweight of the fetuses. For all the tissues analyzed, three modules [i.e., turquoise, red, and green (Fig. S3)] in skeletal muscle were identified to be significantly correlated ( $\mathrm{p}$-value $<0.05$ ) with bodyweight and were enriched with the KEGG pathways associated with overgrowth phenotype (Fig. $2 \mathrm{~A}$ and $\mathrm{B})$. In both turquoise and red clusters, the enriched pathways were involved in cell proliferation and differentiation (e.g., "pathways in cancer" and "cell cycle"), and cell-cell adhesion and fusion (e.g., "focal adhesion", "cell adhesion molecules", and 
"adherens junction") (Fig. 2A and B), while the green module was only enriched with genes associated with the "spliceosome" pathway (Fig. S3).

Fetal myogenesis involves myoblast proliferation/differentiation and fusion of myoblasts into multinucleated muscle fibers (Biressi et al., 2007). Characterization of genes that belong to "pathways in cancer" led us to identify multiple upregulated genes in LOS fetuses that are known to be involved in myoblast proliferation and differentiation (Fig. 2C). For example, IGF1 is a major regulator for skeletal muscle growth and both IGF1 (Schiaffino and Mammucari, 2011) and its downstream signaling component PIK3CD (Schiaffino and Mammucari, 2011) exhibited increased expression levels in LOS \#3 and/or \#4, the two largest fetuses. Besides $I G F 1$, transcript abundance of several other growth factors and their receptors such as FGF1, FGF18, FGFR1, FGFR2, PDGFB, PDGFRA, PDGFRB, TGFB1, and TGFBR2 were also increased in LOS \#3 and/or \#4 (Fig. 2C). These growth factors may work in synergy to promote myoblast proliferation and differentiation (Biressi et al., 2007). In addition, numerous upregulated genes in LOS that belong to the "focal adhesion" and the "cell adhesion molecules" pathways have been reported to play a role in myoblast adhesion and fusion. For example, we found $D O C K 1$, a prototypical member of the family of Rho GTPase activator that is essential for myoblast fusion (Laurin et al., 2008), to be upregulated in both LOS \#3 and \#4 (Fig. 2C). Further, a number of extra cellular matrix genes, reported to be involved in myoblast migration, adhesion, and fusion (e.g., ADAM12, FN1, ICAM1, ITGA3, and ITGA5) (Abmayr and Pavlath, 2012; Thorsteinsdottir et al., 2011) exhibited increased transcript abundance in LOS \#3 and/or \#4 (Fig. 2C). Although it is likely that some of the gene expression changes could be the consequential events of the altered 


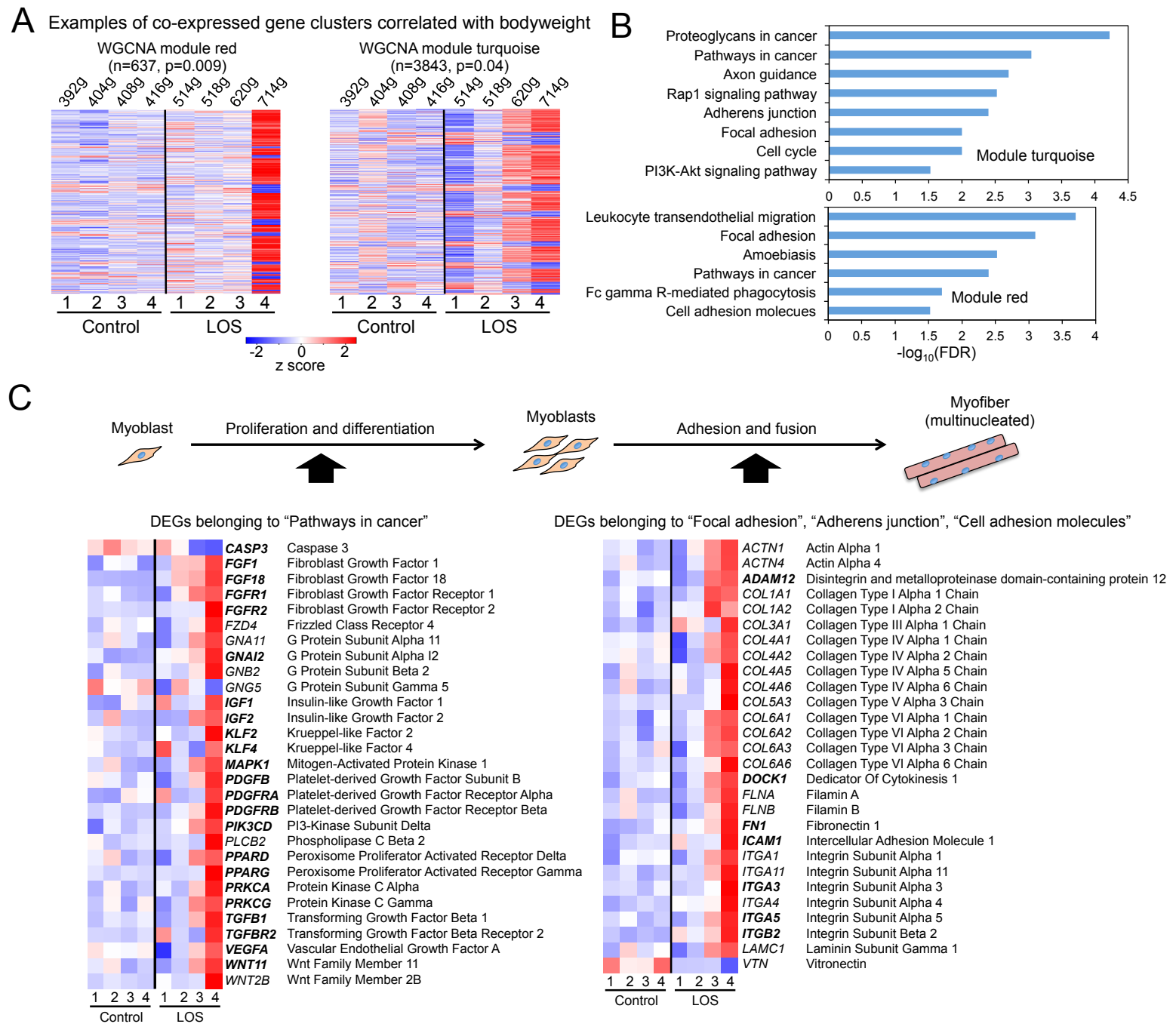

Figure 2. Multiple pathways involved in myogenesis are misregulated in skeletal muscle of

LOS fetuses. A). Heat map profiling of the genes of the WGCNA network module red and turquoise. Red and turquoise modules represent clusters of co-expressed genes that are correlated with bodyweight of the fetuses (details may be found in Figure S2). Shown in the parentheses are the number of genes in each module and p-value of the correlation between the module and bodyweight. The z-score scale represents the mean-subtracted regularized log-transformed read counts. B). Enriched KEGG pathways for module turquoise and red (FDR < 0.05). C). Heat map profiling of the genes that belong to the "pathways in cancer" and the "focal adhesion/adherens junction/cell adhesion molecules" pathways. Bolded genes are those known to be involved in myoblast proliferation and differentiation or fusion of myoblasts into myotubes. 
fetal myogenesis, these data suggest that the misregulated pathways may be responsible for the increased muscle mass and overgrowth phenotype of the LOS fetuses. In summary, gene co-expression network analyses revealed that multiple pathways involved in fetal skeletal muscle development were disrupted in LOS.

Whole genome bisulfite sequencing (WGBS) and read processing

As differential gene expression can be regulated by differential DNA methylation (Smith and Meissner, 2013) and DNA methylation can be altered by the use of ART (de Waal et al., 2012a; de Waal et al., 2012b; Market-Velker et al., 2010; Rivera et al., 2008), we next sought to address whether altered DNA methylation could be the molecular mechanism responsible for the misregulated gene pathways in skeletal muscle of LOS fetuses. To compare the methylome between control and LOS, we generated WGBS data of skeletal muscle of the fetuses used for RNAseq analyses. For each individual, we sequenced $\sim 400$ million $100 \mathrm{bp}$ paired-end reads, which is approximately $26 \times$ coverage of the bovine reference genome (Table S1). The average bisulfite conversion rate was 99.31\% (Table S1). Of note, strong methylation bias was detected at the 5' end of read mate 1 and both ends of read mate 2 (Fig. S4). As these biases could be introduced during the library preparation and/or sequencing steps (Hansen et al., 2012), the first 3 base pairs (bp) and last 2bp of each WGBS read were trimmed and not used for determination of $\mathrm{CpG}$ methylation levels. Following a series of quality filtering steps, $\sim 80 \% \mathrm{CpGs}$ in the reference genome were covered by $\geq 5$ WGBS reads (Table $\mathrm{S} 2$ ). 
Genome-wide CpG methylation landscape and its relationship with transcriptome in fetal skeletal muscle

Prior to making comparisons between LOS and controls, we sought to establish the DNA methylation baseline in bovine fetal skeletal muscle. In controls, the distribution of $\mathrm{CpG}$ sites across different methylation levels exhibited a bimodal pattern (Fig. 3A), which is similar to what have been observed in somatic tissues of human and mouse (Messerschmidt et al., 2014). Further, consistent with the reports that $\mathrm{CpG}$ islands are usually unmethylated (Messerschmidt et al., 2014), the majority of the CpGs within CpG islands in our study were hypomethylated (Fig. 3A and B). In addition, we explored the relationship between the DNA methylome and the transcriptome. We first plotted the average methylation of all the annotated genes along the gene body and $3 \mathrm{~kb}$ upstream of the transcription start site (TSS) and $3 \mathrm{~kb}$ downstream of the transcription end site (TES). The $3 \mathrm{~kb}$ range was chosen because the average DNA methylation beyond this distance was $\sim 0.6-0.7$, which was the overall average methylation of bovine fetal skeletal muscle. As shown in Figure 3C, we found a negative correlation (Spearman rank test, $\mathrm{p}<0.02$ ) between the gene expression level and the level of DNA methylation at regions near the TSS (-500bp to TSS) (Fig. 3C). Furthermore, we queried the relationship between DNA methylation and transcript abundance for genes with different levels of $\mathrm{CpG}$ density at their promoter regions. For genes with high (HCP) and intermediate (ICP) $\mathrm{CpG}$ density promoters, there was a negative correlation (Spearman rank test, $\mathrm{p}<0.02$ ) between the methylation level at promoter regions and the transcript abundance (Fig. 3C). However, low CpG density promoters (LCP) were usually methylated and had no apparent correlation with gene expression levels (Fig. 3C). 
A
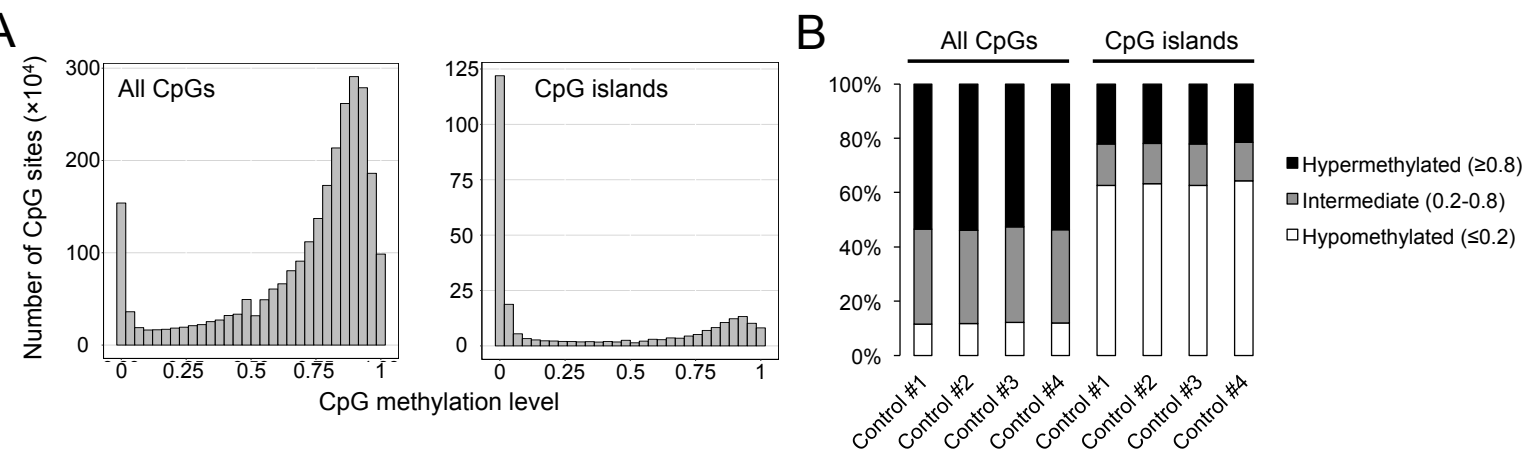

\section{C}
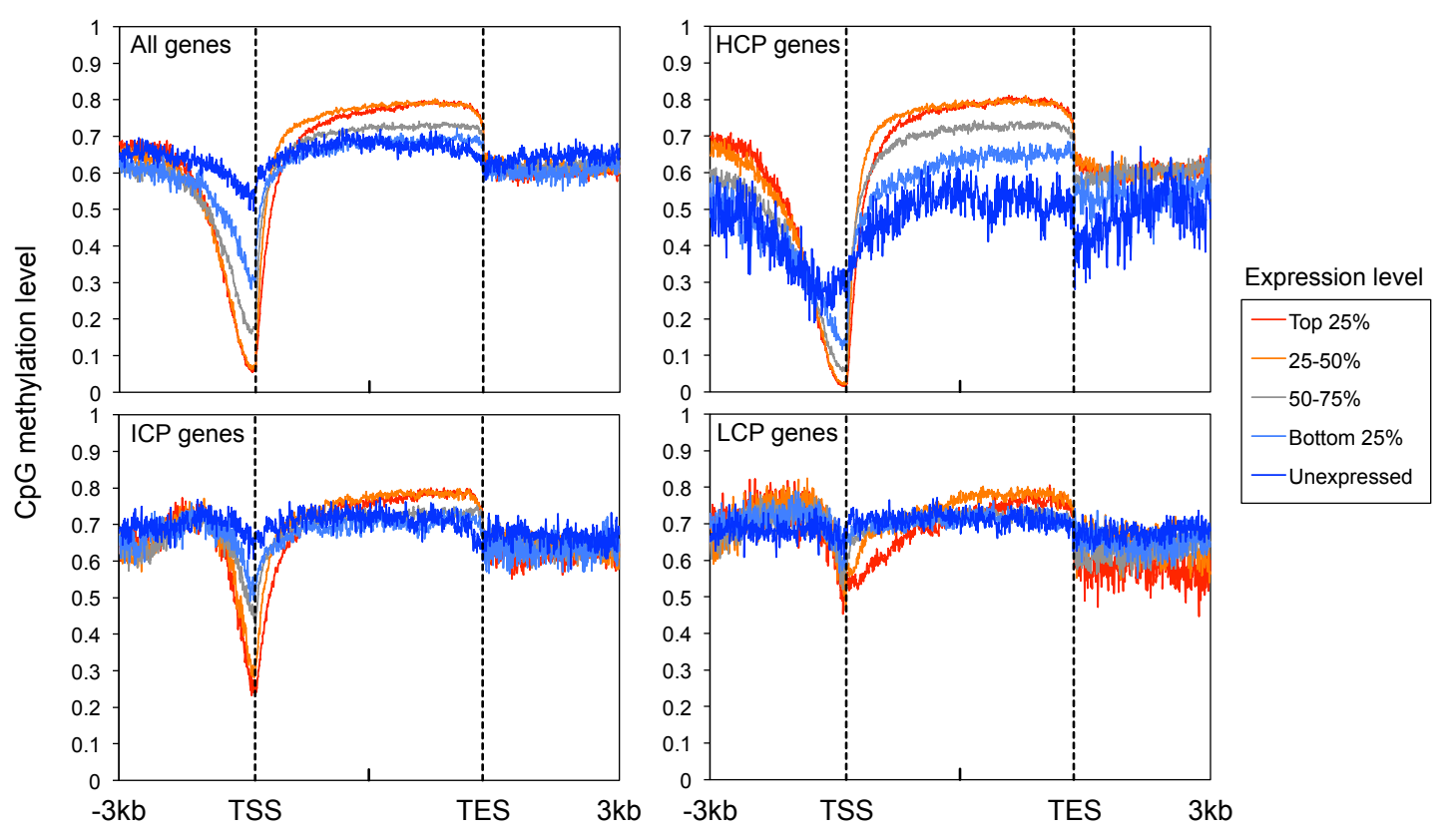

Figure 3. Genome-wide CpG methylation landscape and its relationship to the transcriptome in day $\mathbf{1 0 5}$ skeletal muscle of control fetuses. A). Distribution of CpGs sites across different methylation levels. The methylation level of each $\mathrm{CpG}$ site was calculated as the ratio of the " $\mathrm{C}$ " reads to the sum of the " $\mathrm{C}$ " and " $\mathrm{T}$ " reads. CpGs from all four controls were combined for the generation of this plot. B). Percent of $\mathrm{CpGs}$ at each methylation level in control fetuses. C). Averaged DNA methylation of annotated bovine genes along the gene body and $3 \mathrm{~kb}$ upstream of the TSS and $3 \mathrm{~kb}$ downstream of the TES. Genes were classified into five groups based on their expression levels. HCP: high CpG density promoter; ICP: intermediate $\mathrm{CpG}$ density promoter; $\mathrm{LCP}$ : low $\mathrm{CpG}$ density promoter; TSS: transcription start site; TES: transcription end site. 
Notably, we observed a positive correlation (Spearman rank test, $\mathrm{p}<0.02$ ) between DNA methylation at gene bodies and transcript abundance, especially with HCP genes (Fig. 3C). Overall, in our study, the associations between global DNA methylation and global gene expression were consistent with the findings in human and mouse (Messerschmidt et al., 2014).

Identification and characterization of the ASM regions in bovine fetal skeletal muscle

As only a limited number of imprinted ASM regions have been characterized in bovine (Chen et al., 2015; Chen et al., 2016; Chen et al., 2013; O'Doherty et al., 2012; Robbins et al., 2012), one objective of our study was to identify ASM regions at base resolution in bovine fetal skeletal muscle using WGBS. To distinguish the parental origins of the alleles in the B.t. indicus $\times B$. t. taurus $\mathrm{F}_{1}$ progenies, we obtained heterozygous SNPs of the fetuses from two sources: 1) SNPs identified from the WGBS data using Bis-SNP (Liu et al., 2012) and 2) SNPs identified from the RNAseq data of the $F_{1}$ hybrids (Chen et al., 2016) (Fig. S5). We assigned the WGBS reads overlapping the SNPs to their parental origins based on the genotype of the B. t. indicus sire (Chen et al., 2016) of the $F_{1}$ fetuses. As an initial step to screen CpGs with ASM, allelic WGBS reads were combined from the four controls and CpGs with at least $4 \times$ coverage of each allele $(\mathrm{n}=4,798,414 ; 17.4 \%$ of CpGs in the bovine genome) were subject to Fisher's exact test to determine whether DNA methylation level was significantly different between alleles. In total, 109,794 ASM candidate CpGs were identified with a p-value cutoff of 0.01 [false discovery rate $(\mathrm{FDR}) \approx 0.05$, Fig. S5]. To further minimize the false positive ASM sites, the ASM candidate CpGs were merged into regions using a clustering method 
A

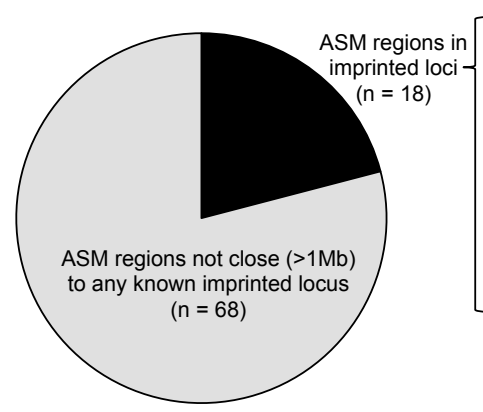

ASM regions previously described in bovine $(n=7)$ :

GNAS DMR, GNASXL DMR, H19/IGF2 DMR ( 2.5kb upstream of H19), IGF2R/AIRN DMR, KVDMR1, MEST DMR, PLAGL1 DMR

Novel ASM regions identified $(n=11)$ :

BEGAIN DMR, DLK1 DMR, DIRAS3 DMR,

GNAS upstream DMR ( 8kb upstream of GNAS), GTL2 DMR,

H19 $1^{\text {st }}$ exon DMR (1st exon of H19), H19 upstream DMR ( 1kb upstream of H19),

MAGEL2 DMR, MEG8 DMR, MGC157368 DMR (PEG3 domain), NAP1L5 DMR

B

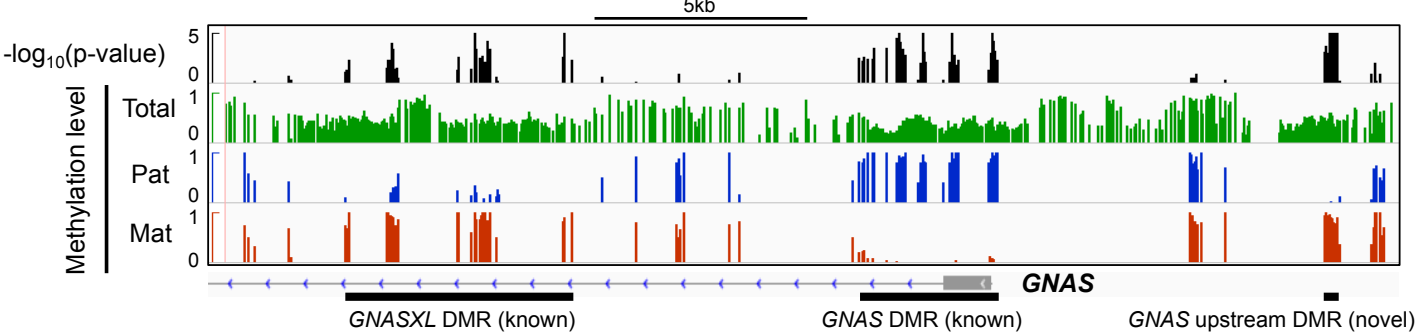

C

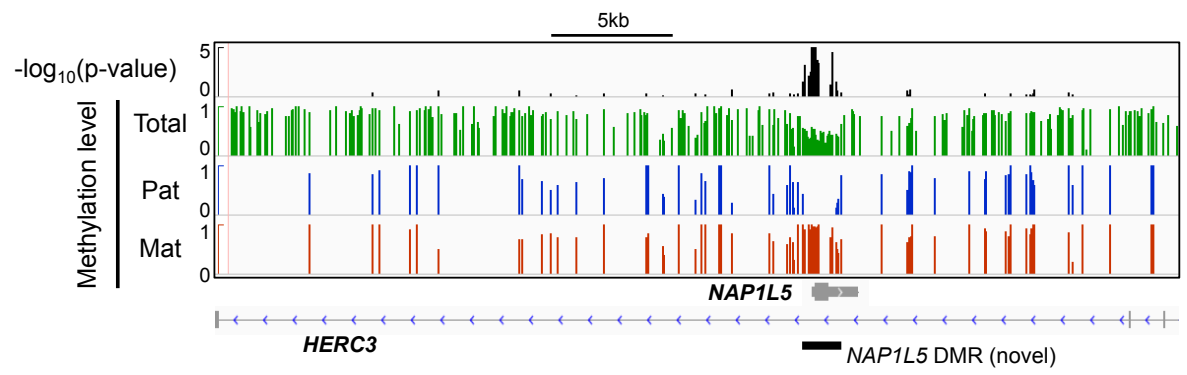

D

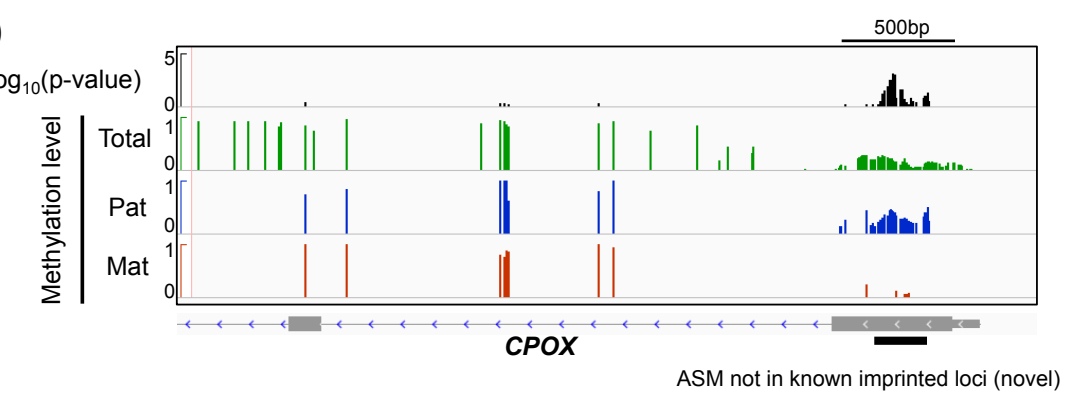

$E$

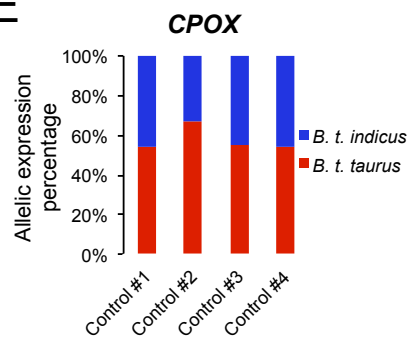

Figure 4. Identification and characterization of ASM regions in skeletal muscle of control fetuses. A). Summary of the ASM regions identified at imprinted and non-imprinted loci. Red color: DMRs with maternal allele-specific methylation; blue color: DMRs with paternal allelespecific methylation. B-D). Genome browser views of the imprinted ASM loci: GNAS/GNASXL and NAP1L5/HERC3 and the non-imprinted CPOX. The $-\log _{10}$ (p-value) for ASM significance (black) and the $\mathrm{CpG}$ methylation levels for total (green), B. t. indicus allele (blue, paternal), and $B$. t. taurus allele (red, maternal) are also shown. Each bar represents a single $\mathrm{CpG}$ site. E). Allelic expression percentage of $C P O X$ in skeletal muscle of the control fetuses. ASM: allele-specific DNA methylation; DMR: differentially methylated region; Mat: maternal allele; Pat: paternal allele. More examples may be found in Figure S7-S8. 
as previously described (Xie et al., 2012). In total, 1,070 ASM CpGs were grouped into 86 discrete genomic regions (Fig. 4A and Table S3).

We manually annotated the identified 86 ASM regions (Table S3) and found that 18 of them were located at known imprinted loci, while others were located $>1 \mathrm{Mb}$ away from any known imprinted gene in bovine (Fig. 4A). The two types of ASM regions are of comparable size; however, the imprinted ASMs have a higher density of CpGs (Fig. S6) located within CpG islands. Both imprinted and non-imprinted ASM loci have overall DNA methylation level around 0.5, but ASM regions at imprinted loci exhibit less variation (range $=0.43$ [imprinted] vs 0.8 [non-imprinted] and $\mathrm{SD}=0.11$ [imprinted] vs. 0.19 [non-imprinted]; Fig. S6). Lastly, the methylation differences between the parental alleles for the ASM regions at imprinted loci are also more dramatic when compared to the ASM regions that are not close to any known imprinted loci (mean $=0.73$ [imprinted] vs. 0.51 [non-imprinted]; Fig. S6).

Of the 18 imprinted ASM regions, seven have been previously described in bovine by us and others including the paternal DMRs: GNAS DMR, H19/IGF2 DMR (Chen et al., 2016; Robbins et al., 2012) and the maternal DMRs: GNASXL DMR, IGF2R/AIRN DMR, KvDMR1, MEST DMR, and PLAGL1 DMR (Chen et al., 2015; Chen et al., 2016; O'Doherty et al., 2012; Robbins et al., 2012) (Fig. 4B and Fig. S7). Of the novel imprinted ASM regions identified in bovine fetal skeletal muscle, eight of them, including NAP1L5 DMR, DIRAS3 DMR, GTL2 DMR and DLK1 DMR (Fig. 4C, Fig. S7), have been previously reported in human and/or mouse (Table S4). The other three novel ASM regions namely, BEGAIN, MEG8, and $M G C 157368$ DMRs, were located within the gene bodies of the known imprinted genes in bovine (Table S3). All 
the ASM regions described above could be associated with the nearby imprinted transcriptional activities previously reported by us (Chen et al., 2016) with the exception of MEST DMR, for which no heterozygous SNP was identified to enable allelic gene expression analyses.

We also manually inspected the imprinted ASM regions previously described in human and/or mouse in our bovine WGBS data. We found that five ASM regions (i.e., GRB10 DMR, H13 DMR, NNAT DMR, PEG3 DMR, and PEG13 DMR) were absent in this study due to either poor SNP coverage or low read coverage (Table S4). A less stringent criterion (i.e., $3 \times$ coverage per allele instead of $4 \times$ ) for ASM calling could lead to the identification of another three DMRs (i.e., INPP5F DMR, PEG10 DMR, and SNRPN DMR) (Table S4). Lastly, we found that some imprinted DMRs in human and/or mouse were simply not subject to ASM in bovine fetal skeletal muscle as these loci were either hypomethylated (e.g., RASGRF1 DMR, ZRSR1/IMPACT1 DMR, and COMMD1 DMR) or partially methylated on both parental alleles (e.g., MKRN3 DMR and GPR1/ZDBF2 DMR) (Table S4).

For the 68 ASM regions not adjacent to any known imprinted locus, we sought to identify genes subject to allele-specific expression in the nearby regions. As shown in Table S3, of the 68 ASM regions, 20 were intergenic ( $>5 \mathrm{~kb}$ away from any annotated gene) while the other 48 regions could be associated with nearby genes. For the 48 genes, parental-allele-specific read counts for 24 genes could be obtained from our previous study (Chen et al., 2016). We found that all of these genes exhibited biallelic gene expression and had no association with the nearby ASM regions (shown in in Fig. 4D and E, and Fig. S8 are five examples). The remaining 24 genes were either lowly 
expressed in skeletal muscle or had no heterozygous SNPs available to ascertain the parental alleles. It is possible that some of these ASM regions are associated with tissuespecific or transcript-isoform-specific monoallelic gene expression.

\section{Lack of direct associations between DMRs and DEGs in LOS}

As for the DEG analysis, each LOS fetus was analyzed independently and compared to the average of all four controls to identify DMRs using the Bsseq R package (Hansen et al., 2012). The hierarchical clustering of CpG methylation within DMRs showed that control fetuses clustered together and were separated from the LOS fetuses (Fig. 5A). The numbers of DMRs identified were similar for the four LOS fetuses with most DMRs identified in LOS \#1 (Fig. 5B). Notably, we identified loss of DNA methylation of KvDMR1 in LOS\#1 and \#4 (Fig. 5C), indicating the validity of the statistical method used for DMR determination, as we have previously identified these specific epimutations by the bisulfite polymerase chain reaction (PCR), cloning, and sequencing (Chen et al., 2013). However, our previous study only reported a DMR with a size of only $385 \mathrm{bp}$ ( $37 \mathrm{CpGs}$ ) while the WGBS analyses allowed us to identify methylation differences between control and LOS across the entire CpG island (1957bp, 207 CpGs, UMD3.1 Chr29: 49,552,845-49,554,801). Further characterization of the DMRs revealed that most of them contained less than 20 CpGs and were of methylation differences between 0.2-0.5 (Fig. 5D).

We then characterized the genomic distances between the identified DMRs and the DEGs, in order to determine whether any of the DMRs could be linked to aberrant gene expression in LOS. We first queried the genomic regions within $5 \mathrm{~kb}$ range of the 


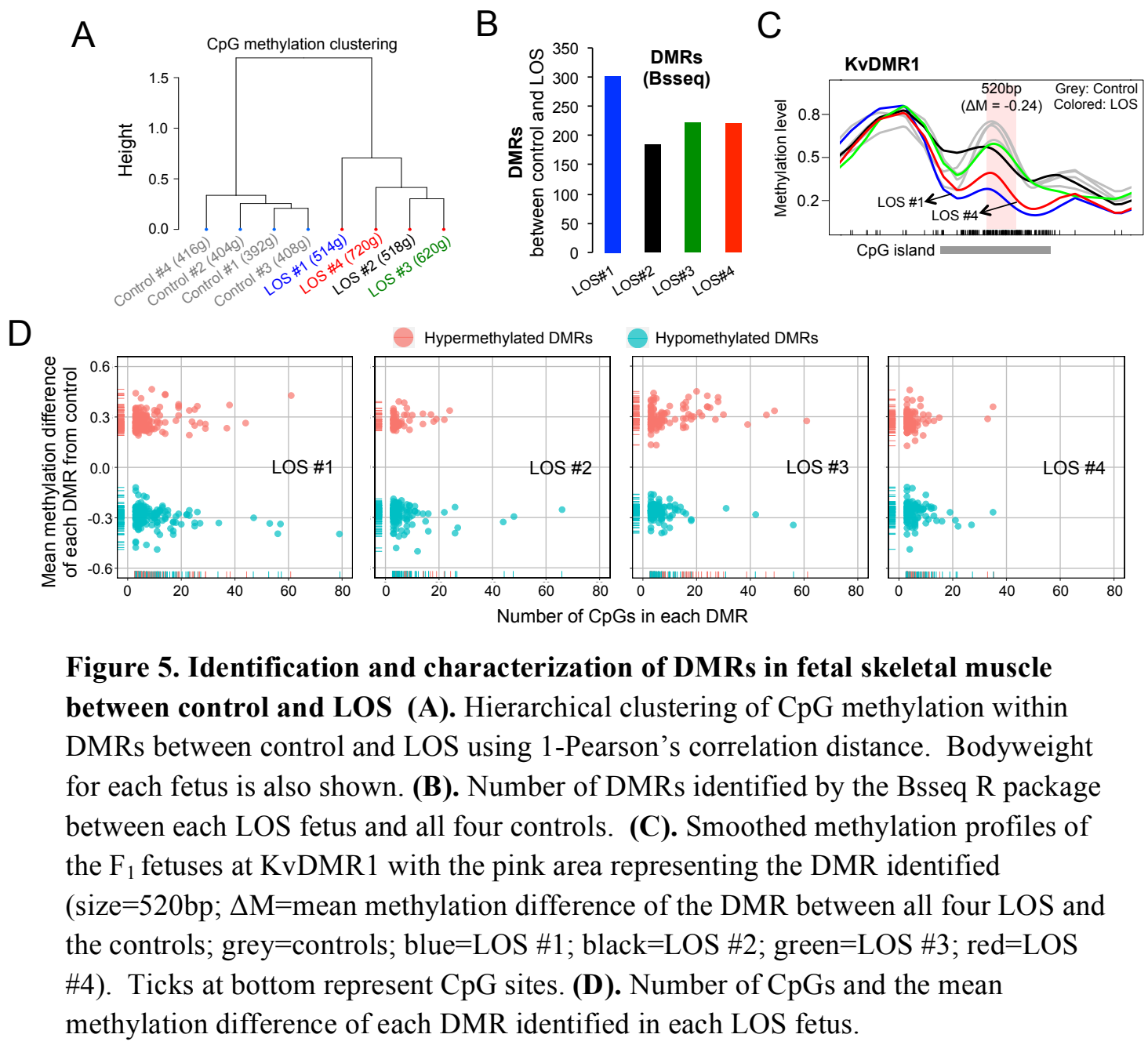


DEGs and found that on average only $\sim 1.5 \%$ of DEGs in each LOS fetus could be associated with the identified DMRs (Fig. 6A). Increasing the range queried from $5 \mathrm{~kb}$ to $20 \mathrm{~kb}$ only added an additional $\sim 0.5 \%$ of DEGs that associate with the identified DMRs (Fig. 6A). To determine whether the numbers of DEGs that are associated with the DMRs were greater than expected by chance, the identified DMRs were shuffled within each chromosome (excluding genome assembly gaps) 1000 times. For each dataset, DEGs were considered to be associated with altered DNA methylation if DMRs were within $5 \mathrm{~kb}$ or $20 \mathrm{~kb}$ range of the DEGs. The expected counts of DEGs were calculated as the average of the 1000 randomized datasets. As shown in Figure 6A, DEGs are associated with DMRs more often than expected by chance in LOS \#1, \#3, and \#4 but not in $\operatorname{LOS} \# 2$.

We found that 1407 DEGs are common between LOS \#3 and \#4 (the two largest LOS fetuses) and we sought to determine if differential DNA methylation can explain misexpression of these genes (Fig. 2C). Only 14 DMRs overlapped between LOS \#3 and \#4, and only one of these was located within $5 \mathrm{~kb}$ of one common DEG (C5H12orf45) and another within $20 \mathrm{~Kb}(R W D D 1)$. We also noted that not all LOS fetuses with aberrant DNA methylation at the same locus had differential gene expression of the associated gene (an example shown in Fig. 6B-C), suggesting that differential DNA methylation is not the sole cause of differential gene expression. Lastly, minor methylation differences were detected in the TSS regions $(-1.5 \mathrm{~kb}$ to $+500 \mathrm{bp})$ of the DEGs between control and LOS (Fig. 6F and Fig. S9). In summary, only a very small percent DEGs in fetal skeletal muscle could be directly associated with the DMRs between control and LOS. 

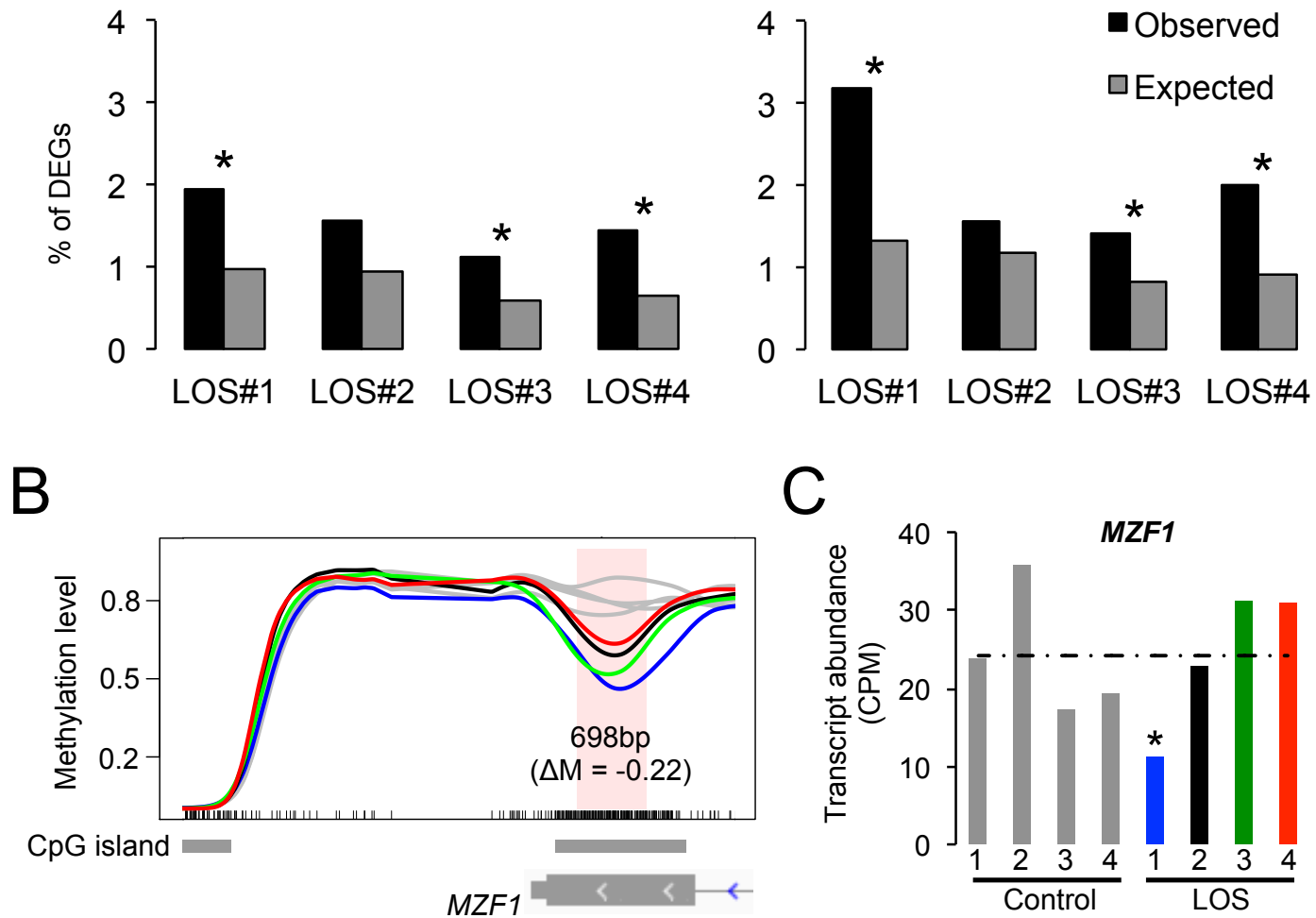

$D$
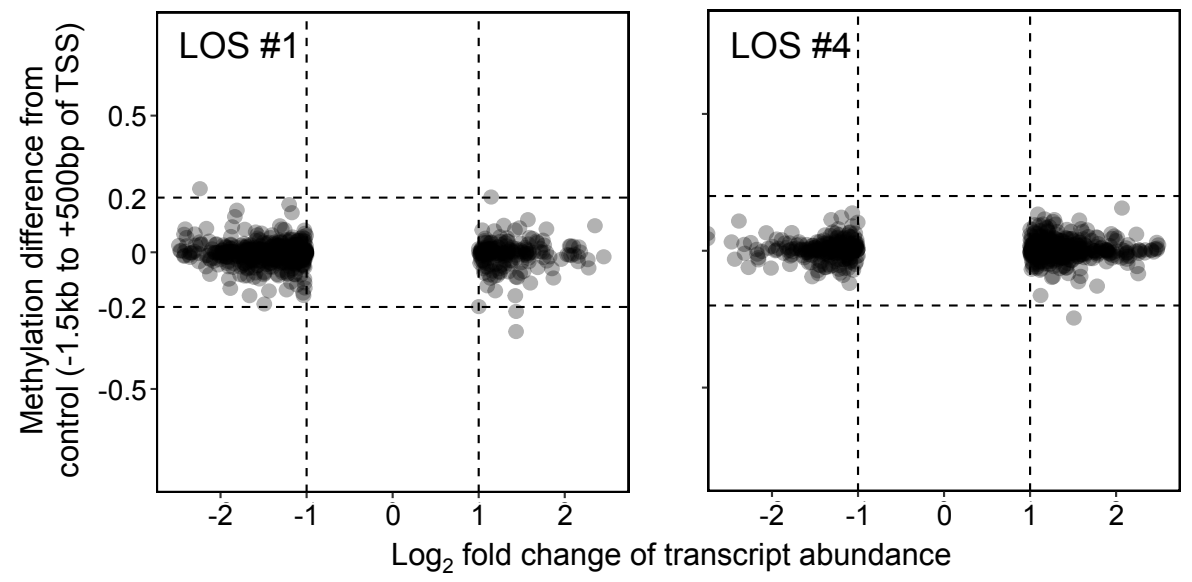

Figure 6. Lack of associations between the DEGs and the DMRs in skeletal muscle of the LOS fetuses. (A). Percentage of DEGs that are associated with the DMRs in LOS fetuses. A DMR is considered to be associated with a DEG if it is less than $5 \mathrm{~kb}$ or $20 \mathrm{~kb}$ away from the DEG. To determine whether the number of DEGs associated with DMRs were greater than expected by chance, DMRs were shuffled within each chromosome 1000 times and the expected numbers of DEGs associated with the DMRs were calculated as the average of 1000 datasets and were used in chi-square tests $\left({ }^{*} p<0.01\right)$. (B-C). Examples of lack of association between DEGs and DMRs when the DMRs are within $5 \mathrm{~kb}$ range of the DEGs. For (B), the details may be found in Fig. 5. Panel (C) shows the transcript abundance of $M Z F 1$ in all fetuses (asterisk: edgeR FDR < 0.05). DEG: differentially expressed genes. (D). Mean methylation level differences of the DEGs between control and LOS fetuses at the TSS region $(-1.5 \mathrm{~kb}$ to $+500 \mathrm{bp})$. Only DEGs that had at least 2-fold change are shown here. 
Association of aberrant DNA methylation and differential gene expression at IGF2R/AIRN and MAGEL2 imprinted loci

Insulin-like growth factor 2 receptor (IGF2R) is a scavenger receptor of the fetal growth factor IGF2, and is maternally expressed in cow (Bebbere et al., 2013; Chen et al., 2015) (Fig. 7A) and mouse (Barlow et al., 1991), but exhibits polymorphic imprinting in human (Xu et al., 1993). In this study, IGF2R exhibited decreased transcript abundance in three out of four LOS fetuses (Fig. 7C), and this differential gene expression was associated with the altered DNA methylation at DMR1 and DMR2 of the IGF2R locus (Fig. 7A-B, Fig. S9). In all four LOS fetuses, we found loss of methylation of DMR2, which is located in the second intron of IGF2R with loss of methylation in all four LOS fetuses (Fig 7B). Normally, DMR2 was methylated on the maternal allele and unmethylated on the paternal allele. Hypomethylation of DMR2 has also been reported in sheep LOS (Young et al., 2001), suggesting the $I G F 2 R$ locus is similarly misregulated in these two species. DMR1, which is close to the promoter region of $I G F 2 R$, is normally partially methylated on the paternal allele and unmethylated on the maternal allele. In our study, DMR1 was hypermethylated in LOS (Fig. 7A-B).

In mice, paternal silencing of $I G F 2 R$ is mediated by the paternally expressed long non-coding RNA $A I R N$, which is transcribed in the antisense direction to IGF2R and is regulated by DMR2 of $I G F 2 R$ (Wutz et al., 1997). Thus, we reasoned that hypomethylation of DMR2 can lead to biallelic expression of $A I R N$, which represses the expression of $I G F 2 R$ on both alleles and results in the decreased level of IGF2R in LOS fetuses. However, contrary to our expectation, $A I R N$ was biallelically expressed in both control and LOS fetal skeletal muscle (Fig. 7D), suggesting that down-regulation of 
A

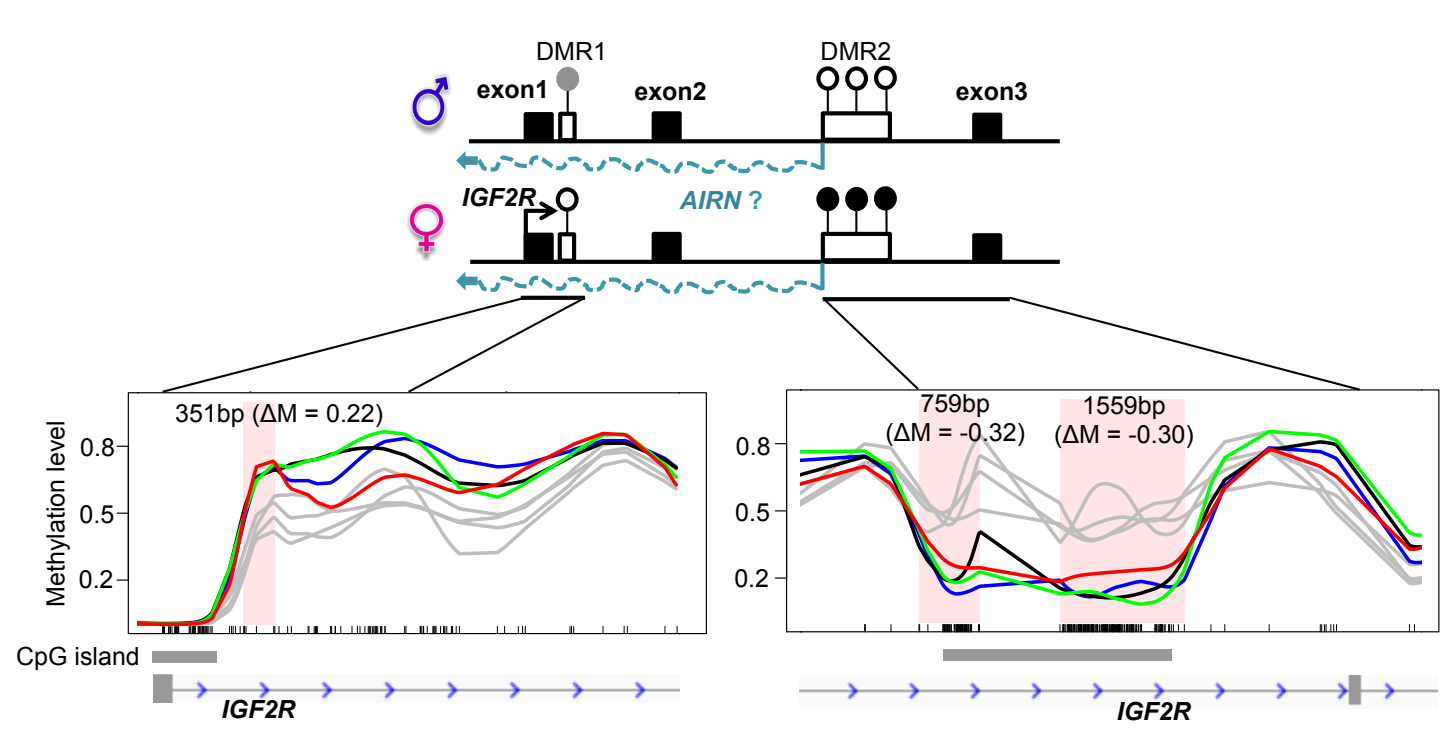

C

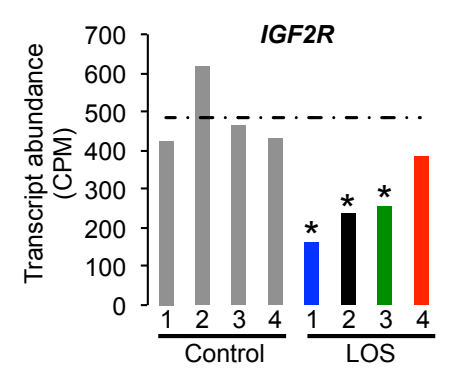

Allelic expression analyses of AIRN

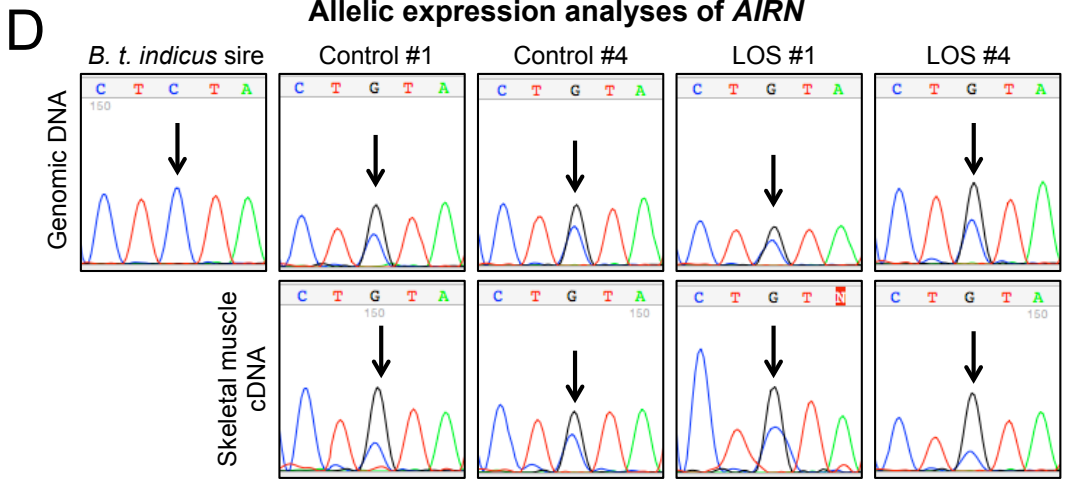

$\mathrm{E}$

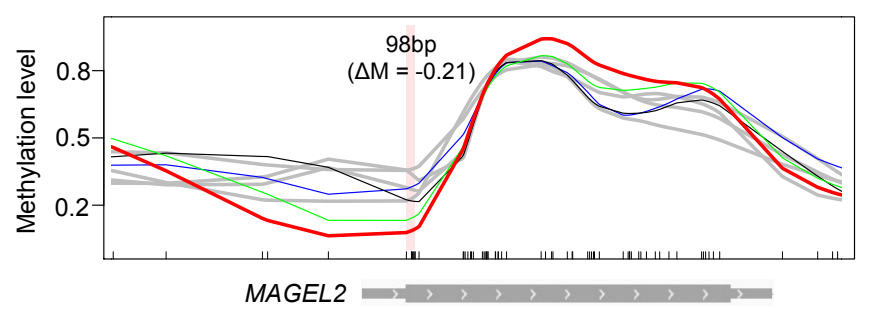

$\mathrm{F}$

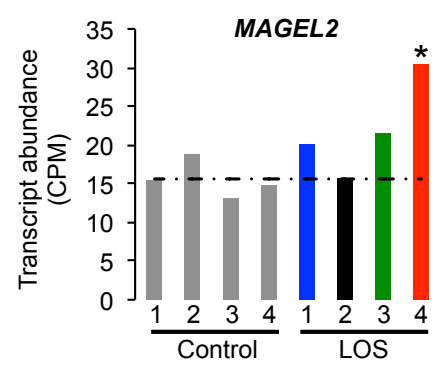

Figure 7. DNA methylation and differential gene expression at IGF2R/AIRN and MAGEL2 imprinted loci. A). Schematics of the $I G F 2 R / A I R N$ locus. Bent arrows represent the transcription start sites. White boxes represent the ASM regions (white lollipops = unmethylated; grey lollipop = partially methylated; black lollipops = methylated). B). Smoothed methylation profiles for the two DMRs at IGF2R locus between control and LOS fetuses. The pink areas represent the DMR identified $(\triangle \mathrm{M}=$ mean methylation difference of the DMR between all four LOS and the controls;

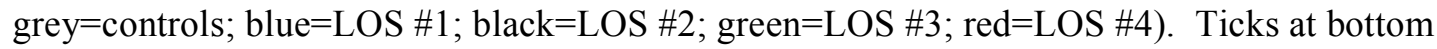
represent CpG sites. C). Transcript abundance of IGF2R in control and LOS fetuses (asterisk: edgeR FDR $<0.05$, LOS vs control). D). Allelic expression analyses for $A I R N$ by Sanger sequencing. The SNP site is indicated by an arrow. E) Smoothed methylation profile of MAGEL2 locus. The labels are as panel B. F). Transcript abundance of MAGEL2 in the $\mathrm{F}_{1}$ fetuses (asterisk: edgeR FDR $<0.05$ ). 
$I G F 2 R$ is not mediated by biallelic expression of $A I R N$. Nevertheless, decreased transcript abundance of $I G F 2 R$ was associated with a hypomethylated DMR2 and hypermethylated DMR1 in LOS fetuses.

In addition to $I G F 2 R$, we detected an association between differential gene expression and differential DNA methylation at the MAGEL2 locus. MAGEL2 is a paternally expressed gene (Chen et al., 2015) with its promoter methylated on the maternal allele but unmethylated on the paternal allele (Table S3). As shown in Fig. 7E$\mathrm{F}$, the increased transcript abundance of MAGEL2 in LOS \#4 is associated with the loss of DNA methylation at its promoter region.

\subsection{Discussion}

Since BWS was first described in the early 1960s (Weksberg et al., 2005) and the first cases of ART-induced LOS were reported in 1995 (Farin and Farin, 1995), the main focus in both fields has been to understand how loss-of-imprinting alone can contribute to the complex and variable phenotypic features of these overgrowth conditions. In human, the epimutation and genetic alteration of the two imprinted loci (i.e., $K C N Q 1$ and $H 19 / I G F 2$ loci) affect nearly $70 \%$ of BWS patients with the hypomethylation at KvDMR1 accounting for $\sim 50 \%$ of all BWS cases (Eggermann et al., 2016). In addition, loss-of-imprinting at multiple loci was observed in $\sim 25 \%$ of BWS cases with epimutation at the KCNQ1 locus (Eggermann et al., 2016). Similarly in bovine, we have also shown that LOS exhibits loss-of-imprinting at multiple loci including the $K C N Q 1$ locus (Chen et al., 2015; Chen et al., 2013). However, to date, there has been no report of molecular 
features that can consistently and reliably diagnose and predict these syndromes and the associated variable phenotypes. Here we advance the field by describing the altered transcript abundance of genes beyond imprinted loci in LOS fetuses, showing that lossof-imprinting is not be the only molecular lesion of this condition.

In this study, WGCNA and KEGG pathway analyses identified that multiple pathways involved in myoblast proliferation and fusion of myoblasts into myotubes are misregulated in skeletal muscle of LOS fetuses. This finding is consistent with the previous report that short-term culture of ovine embryos can affect myogenic programming and the altered myogenesis may contribute to the large muscles observed in the oversized fetuses (Maxfield et al., 1998). In addition to skeletal muscle, altered transcript abundance of genes was also detected in liver, brain, and kidney of the LOS fetuses. Interestingly, tissue specific alterations in transcript abundance varied between LOS fetuses. For example, LOS \#1 had the most DEGs in liver, LOS \#3 in kidney and LOS \#4 in muscle. This observation is supported by findings in BWS in which different individuals have different susceptibility for Wilms tumor of the kidney or hepatoblastoma (Eggermann et al., 2016). Future studies with in depth characterization of the histology and the transcriptome of the tissues at various developmental stages of LOS will better elucidate relationships between the altered gene pathways and the potential disturbed development of these organs.

An interesting finding of this study was that only a small percent of DEGs could be linked to the identified DMRs between control and LOS. Recently, it has been shown that histone modifications such as $\mathrm{H} 3 \mathrm{~K} 27 \mathrm{me} 3$ at distal regions in oocytes can escape epigenetic reprogramming and maintain inheritance during the pre-implantation 
development (Zheng et al., 2016). It is possible that molecular marks like H3K27me3 in bovine may act as an epigenetic memory of the use of ART procedures and mediate the differential gene expression and the aberrant development in later stages. Alternatively, the DEGs identified in this study could represent the downstream effect of the aberrant expression of developmentally important genes affected by ART during pre-implantation development. In summary, our study demonstrates that altered transcriptome in LOS skeletal muscle is largely uncoupled to DNA methylome epimutations and we submit that caution should be exercised in interpreting results from DNA methylation data alone when studying the etiology of these overgrowth syndromes.

In addition to providing novel insights on the etiology of LOS, our study characterized the cytosine methylation landscape at base resolution in bovine. Consistent to what has been previously reported for other mammals, $\mathrm{CpG}$ islands in bovine are hypomethylated and a negative association between DNA methylation and gene expression levels are observed at HCP and ICP, but not LCP promoters (Messerschmidt et al., 2014). Furthermore, we have identified and characterized novel ASM at both imprinted and non-imprinted loci in bovine. For the imprinted loci, we have expanded the number of imprinted ASM in bovine by describing several novel DMRs such as DIRAS3 DMR, GTL2 DMR, and NAP1L5 DMR. Moreover, we have shown that some known imprinted DMRs in human and/or mouse (e.g., the L3MBTL DMR in human and the primary DMRs RASGRF1 and IMPACT in mouse) are not subject to ASM in fetal bovine skeletal muscle, suggesting species-specific imprinting of these genes. Lastly, we have identified ASM regions at non-imprinted loci which others (Tycko, 2010) have proposed may be the result of cis-acting regulatory polymorphisms. It should be noted 
that ASM regions identified in this study are not comprehensive because we could not assign all WGBS reads covering CpGs to either parental alleles due to the lack of informative SNPs. In future studies, analyses of the $\mathrm{F}_{1}$ hybrids of different crosses may further improve the identification of ASM CpG sites.

In conclusion, we have shown that misregulation of non-imprinted genes in addition to loss-of-imprinting characterizes to the ART-induced overgrowth syndrome and have demonstrated that most of the aberrant gene expression in fetal skeletal muscle is not associated with aberrant DNA methylation.

\subsection{Materials and Methods}

RNAseq data analyses

The production of the B.t. indicus $\times$ B.t.taurus $\mathrm{F}_{1}$ fetuses and collection of the fetal tissues were previously described by us (Chen et al., 2015). All animal procedures were performed at TransOva Genetics by veterinarians, and all procedures were approved by their animal care and use committee. RNAseq data of the control and LOS fetuses were obtained from one of our previous studies and were processed as previously described (Chen et al., 2015; Chen et al., 2016). DEGs between each LOS fetus and the mean of all four controls were determined with the consideration of variance among the controls using the edgeR package (Anders et al., 2013; Robinson et al., 2010). The WGCNA package (Langfelder and Horvath, 2008) was used to construct a weighted gene co-expression network for the skeletal muscle of the four control and four LOS fetuses. Further, the identified network modules were correlated with the bodyweight to identify 
genes in modules most likely responsible for the altered bodyweight. In addition, KEGG pathway analyses for the significant network modules and the DEGs in LOS fetuses were performed using the Database for Annotation, Visualization and Integrated Discovery (DAVID) (Huang da et al., 2009).

\section{WGBS library preparation and sequencing}

Genomic DNA isolated from skeletal muscle of four control and four LOS day $\sim 105$ B.t.taurus $\times$ B.t.taurus $\mathrm{F}_{1}$ female fetuses (Chen et al., 2015) were subject to WGBS analyses. The WGBS libraries were generated using the NEBNext Ultra DNA library Prep Kit for Illumina (NEB E7370) as per the manufacturer's instructions. Prior to sodium bisulfite mutagenesis, $0.5 \%$ unmethylated Lambda DNA was added to the bovine DNA to act as an internal control to monitor the bisulfite conversion rate. Each WGBS library was sequenced using Illumina sequencing technology to generate 100bp paired-end reads. The raw FASTQ files are publically available at Gene Expression Omnibus (GEO accession no. GSE93775).

\section{WGBS data analyses}

Following adaptor removal and quality trimming, WGBS read pairs were aligned to the bovine reference genome assembly UMD3.1 using Bismark (version: 0.15.0) (Krueger and Andrews, 2011) with the default parameters. Only uniquely aligned reads were used for subsequent analyses. In order to distinguish the $\mathrm{C}>\mathrm{T}$ SNPs from $\mathrm{C}>\mathrm{T}$ substitutions that were caused by sodium bisulfite conversion in the $\mathrm{F}_{1}$ fetuses, Bis-SNP (version: 0.82.2) (Liu et al., 2012) was used to perform the SNP calling. Only CpG sites that showed consensus $\mathrm{CpG}$ context for both parental alleles (i.e., do not overlap any 
SNPs identified by Bis-SNP) were used for the subsequent analyses. The methylation level was determined using the reads from both forward and reverse strands covering the same symmetric $\mathrm{CpG}$ site. The methylation level was calculated as: (number of "C" reads)/(number of "C" reads + number of "T" reads).

In order to identify ASM regions, the WGBS read pairs overlapping SNPs were assigned to their parental origin based on the genotype of the B. t. indicus sire (Chen et al., 2016). Further, the allelic WGBS reads were pooled from four controls to determine the sequencing depth and only CpGs that had at least $4 \times$ coverage of each allele were used for Fisher's exact test. To estimate the FDR, WGBS read pairs overlapping SNPs were randomly assigned to be either B. t. indicus or B. t. taurus in origin in order to generate a randomized dataset of ASM sites (Xie et al., 2012). ASM CpGs were identified using a p-value of 0.01, resulting in 109,794 ASM sites with a FDR of $\sim 5 \%$ (compared to the $5507 \mathrm{CpGs}$ identified as ASM sites in the permutated datasets). Lastly, the identified ASM sites were clustered into regions as previously described (Xie et al., 2012). Methylkit R package (Akalin et al., 2012) was used to perform the hierarchical clustering of the methylation profiles. The DMRs between each LOS fetus and all four controls were identified using the Bsseq R package (Hansen et al., 2012).

To estimate the expected number of DEGs associated with DMRs, the identified DMRs were randomly shuffled within each chromosome using "shuffleBed" function from BedTools (Quinlan and Hall, 2010). The UMD3.1 genome assembly gaps from UCSC genome Browser were excluded from the potential shuffled positions. Shuffling was performed 1000 times and for each dataset, a DEG was considered to be associated with a DMR if the DMR falls into the DEG coordinates that had been extended by $5 \mathrm{~kb}$ or 
$20 \mathrm{~kb}$. The averaged DEG counts of the 1000 datasets were used as the expected count for chi-square test.

\section{Statistical analyses and data visualization}

Statistical analyses were implemented with R (http://www.r-project.org/). Heat maps were generated with the R function "heatmap.2". The averaged methylation level of the annotated genes were generated using deepTools (Ramirez et al., 2014). For ASM regions, bed files were converted to the bigwig format and visualized as custom tracks in the Integrative Genomics Viewer (IGV) (Robinson et al., 2011).

\section{Allelic expression analyses of AIRN}

Both DNA and cDNA of the $\mathrm{F}_{1}$ fetuses and DNA of the sire were amplified by PCR followed by Sanger sequencing to ascribe the parental origin of the transcript. Nucleic acid isolation, cDNA synthesis, and PCR amplifications were as previously reported (Chen et al., 2015).

\subsection{Acknowledgements}

We thank Dr. Susanta Behura for the critical reading of the manuscript. This work was supported by the University of Missouri Research Board (RB 16-07 HAGEN), National Science Foundation (NSF Award No. 1615789), National Institute of Health (Grant 5R21HD062920), and the Food for the $21^{\text {st }}$ Century Program at the University of Missouri. 


\title{
3.7 Supplemental information for Chapter 3
}

\section{Supplemental materials and methods}

\author{
WGBS library preparation and sequencing
}

Genomic DNA isolated from skeletal muscle of four control and four LOS d105 B. t. taurus $\times$ B. $t$. taurus $\mathrm{F}_{1}$ fetuses were subject to WGBS analyses. For library preparation, $1 \mu \mathrm{g}$ bovine DNA was subject to sodium bisulfite mutagenesis using NEB EpiMark Bisulfite Conversion Kit (NEB E3318) according to the manufacturer's instructions. Prior to the sodium bisulfite reaction, 5ng (0.5\%) unmethylated cl857 Sam7 Lambda DNA (48,502bp, Promega D1521) was combined with bovine DNA to act as an internal control to monitor the bisulfite conversion rate. The bisulfite conversion rate was calculated as the percentage of thymines sequenced at the cytosine reference positions in the Lambda genome. The WGBS libraries were generated using the NEBNext Ultra DNA library Prep Kit for Illumina (NEB E7370) as per the manufacturer's instructions. Each WGBS library was sequenced in two lanes with 100bp paired-end reads on an Illumina HiSeq 2000 platform. For Control \#4 fetus, one lane of the WGBS library was sequenced on an Illumina HiSeq 2500 platform.

\section{WGBS reads trimming and alignment}

Raw WGBS read pairs were subject to quality trimming prior to alignment to the reference genome. The Cutadapt program (version: 1.9.1) (Martin, 2011) was used to remove the adaptor sequences in the read pairs. For base quality trimming, DynamicTrim (version 3.1) (Cox et al., 2010) was used to obtain the longest segment of

the read in which each base has $<1 \%$ error rate. Following quality trimming, WGBS read 
pairs were aligned to the bovine reference genome assembly UMD3.1 and the Lambda genome using Bismark (version: 0.15.0) (Krueger and Andrews, 2011) with the default parameters. Only uniquely aligned read pairs that had the expected read mate orientation and expected ranges of insert size were retained for further analyses.

\section{Identification of SNPs using WGBS data}

In order to distinguish the $\mathrm{C}>\mathrm{T} \mathrm{SNPs}$ in the $\mathrm{F}_{1}$ fetuses from the $\mathrm{C}>\mathrm{T}$ substitutions that are caused by sodium bisulfite conversion, Bis-SNP (version: 0.82.2)(Liu et al., 2012) was used to perform the SNP calling using the WGBS data. In brief, the uniquely aligned WGBS read pairs were subject to several post-alignment processes prior to the SNP calling including PCR duplicates removal, insertions/deletions re-alignment, and base quality recalibration. Known insertions/deletions (indel) and known SNPs downloaded from Ensembl and RefSeq FTP sites were supplied for the indel realignment and the base quality recalibration (ftp://ftp.ensembl.org/pub/release78/variation/vcf/bos_taurus/; ftp://ftp.ncbi.nlm.nih.gov/snp/organisms/cow_9913/VCF/; ftp://ftp.ncbi.nlm.nih.gov/snp/organisms/cow_30522/). In addition, the SNPs identified from the DNA sequencing data (Chen et al., 2016) of the sire of the B. t. indicus X B. t. taurus $\mathrm{F}_{1}$ fetuses were also supplied for the base quality recalibration process. After processing the uniquely aligned WGBS read pairs, Bis-SNP was used to identify SNPs with the exclusion of first $3 \mathrm{bp}$ and last $2 \mathrm{bp}$ of the both read mates using the option "trim5 3 -trim 32 " as these read positions were associated with strong methylation bias (Fig. S1). 


\section{Determination of the methylation level of each $C p G$ site}

Only $\mathrm{CpG}$ site that show consensus $\mathrm{CpG}$ context for both parental alleles (i.e., do not overlap any SNPs identified by Bis-SNP) were used for the subsequent analyses. As CpG methylation is symmetric, the methylation level was determined using the reads from both forward and reverse strands covering the same symmetric $\mathrm{CpG}$ site. The methylation level was calculated as: (number of "C" reads)/(number of "C" reads + number of "T" reads). For most analyses, only CpGs covered by at least by five reads were used for the calculation of the methylation level. The exception is the allelespecific methylation analyses, in which only CpGs covered at least by 8 reads (four reads per parental allele) were included for the statistical test. For reads with PCR duplicates, only one of them was randomly choosed for the determination of the methylation level.

\section{Identification of the ASM regions}

Heterozygous SNPs of the fetuses were obtained from two sources for the determination of parental allele origin: 1) SNPs identified from the WGBS data using Bis-SNP (Liu et al., 2012); 2) SNPs identified from the RNA-seq data of the $\mathrm{F}_{1}$ hybrids (Chen et al., 2016). The WGBS read pairs overlapping SNPs were assigned to their parental origins based on the genotype of the B. t. indicus sire (Chen et al., 2016). If the SNP included a cytosine, its bisulfite converted form thymine was also considered. Further, the allelic WGBS reads were pooled from four controls to determine the sequencing depth and only CpGs that had at least $4 \times$ coverage of each allele were used for Fisher's exact test. To estimate the FDR, WGBS read pairs overlapping SNPs were permutated for their parental origin by randomly assigning them to either B. t. indicus or 
B. t. taurus alleles. The ASM for each $\mathrm{CpG}$ in the permutated dataset was assessed using Fisher's exact test. The permutation was performed five times and the numbers of identified ASM CpGs at different p-value cutoff were averaged. ASM CpGs were identified using a p-value of 0.01 , resulting 109,794 ASM sites with a FDR of $\sim 5 \%$ as simply by chance 5507 ASM sites can be identified in the permutated datasets. Lastly, the identified ASM sites were clustered into regions as previously described (Xie et al., 2012). In brief, significant ASM sites within $2 \mathrm{~kb}$ were merged as DMR candidates. For each candidate, at least five ASM sites were required and at least 75\% CpGs should show consistent methylation bias toward the same allele. The clustered DMRs were also manually inspected and annotated.

\section{Association between DEGs and DMRs in LOS fetuses}

Methylkit R package (Akalin et al., 2012) was used to perform the hierarchical clustering of the methylation profiles using 1-Pearson's correlation distance. The DMRs between each LOS fetus and all four controls was identified using Bsseq R package (Hansen et al., 2012) with the default parameters except that only CpGs with at least 5 reads coverage for each fetus were included for analyses. For comparison, Bsseq was also used to identify DMRs between all four LOS fetuses and the controls.

RNA-seq data of the control and LOS fetuses were obtained from one of our previous studies and were processed as previously described (Chen et al., 2015; Chen et al., 2016). DEGs between each LOS fetus and all four controls were determined using the edgeR package (McCarthy et al., 2012; Robinson et al., 2010). In brief, for each LOS fetus, a DGElist was created with the gene read counts of the four controls and the LOS 
fetus. Following normalization (i.e., function "calcNormFactors") and parameters estimation (functions "estimateGLMTrendedDisp" and "estimateGLMTagwiseDisp"), functions "glmFit" and "glmLRT" were used to identify genes with differential transcript abundance between the LOS and the mean of the four controls (FDR $<0.05$ ). To determine whether any DMRs are associated with DEGs, $5 \mathrm{~kb}$ or $20 \mathrm{~kb}$ regions surrounding the DEGs (including the gene body) were used to intersect with the DMRs using "intersectbed" from bedtools (Quinlan and Hall, 2010). The methylation level of the TSS region $(-1.5 \mathrm{~kb}$ to $+500 \mathrm{bp})$ was calculated by averaging the methylation level of all CpGs with at least five reads coverage.

\section{WGCNA and KEGG analyses}

A weighted gene co-expression network was constructed for the skeletal muscle of the four control and four LOS fetuses using "blockwiseModule" function from the WGCNA package (Langfelder and Horvath, 2008). Further, the identified network modules were linked to the external trait (i.e., bodyweight) to determine which module is significantly correlated to the bodyweight. In addition, KEGG pathway analyses for the significant network modules were performed using the Database for Annotation, Visualization and Integrated Discovery (DAVID) (Huang et al., 2007).

\section{Genomic annotations}

The TSS and TES of the annotated genes were obtained from Bovine Genome Database (http://bovinegenome.org)(Elsik et al., 2015) and were curated with the RNAseq data of brain, kidney, liver, and skeletal muscle using cuffmerge (Trapnell et al., 2010). Annotation of $\mathrm{CpG}$ islands was obtained from UCSC genome browser 
(Rosenbloom et al., 2015), in which the CpG islands are defined as genomic regions that fulfill the following criteria: 1) $>200 \mathrm{bp}, 2$ ) GC content $\geq 50 \%$ and 3 ) the ratio of the observed CpGs to the expected CpGs $(\mathrm{O} / \mathrm{E})>0.6$.

Promoters are defined as $-2 \mathrm{~kb}$ to $+500 \mathrm{bp}$ of the TSS (Liao et al., 2015). High CpG Promoters (HCP) promoters contain a 500bp interval with: 1) GC content $\geq 55 \%$ and 2) the $\mathrm{O} / \mathrm{E}$ ratio $\geq 0.6$ (Mikkelsen et al., 2007). Low CpG Promoters (LCP) promoters do not contain a 500bp interval with the $\mathrm{O} / \mathrm{E}$ ratio $\geq 0.4$ (Mikkelsen et al., 2007). The rest of the promoters are classified as Intermediate CpG Promoters (ICP) promoters (Mikkelsen et al., 2007). The formula used for calculation of the O/E ratio of a sequence segment is as follows: number of CpGs * sequence length / (number of C * number of G) (Gardiner-Garden and Frommer, 1987).

\section{Allelic expression analyses of AIRN}

Both DNA and cDNA of the $\mathrm{F}_{1}$ fetuses and DNA of the sire were amplified by PCR followed by Sanger sequencing to ascribe the parental origin of the transcript. Nucleic acid isolation, cDNA synthesis, and PCR amplifications were as previously reported (Chen et al., 2015). The primers used are as follows: forward (5'AGAAACGGGCACATTCA-3') and reverse (5' GGCAATATTAGAACAGCTAAGAG-3').

\section{Statistical analyses and data visualization}

Statistical analyses such as Fisher's exact test were implemented with R (http://www.r-project.org/). Fig. 2A and C and Fig. S2C-D were generated with R function "heatmap.2". Fig. 3C and 5B were generated using the "computeMatrix" and 
"plotProfile" functions in deepTools (Ramirez et al., 2014). For Fig. 4B-D, Fig. 5C, Fig. S5, Fig. S6A, C, and E, bed files were converted to the bigwig format using the "bedGraphToBigWig" from UCSC Genome Browser database and visualized as custom tracks in the Integrative Genomics Viewer (IGV) (Robinson et al., 2011). 


\section{Supplemental figures and tables for Chapter 3}
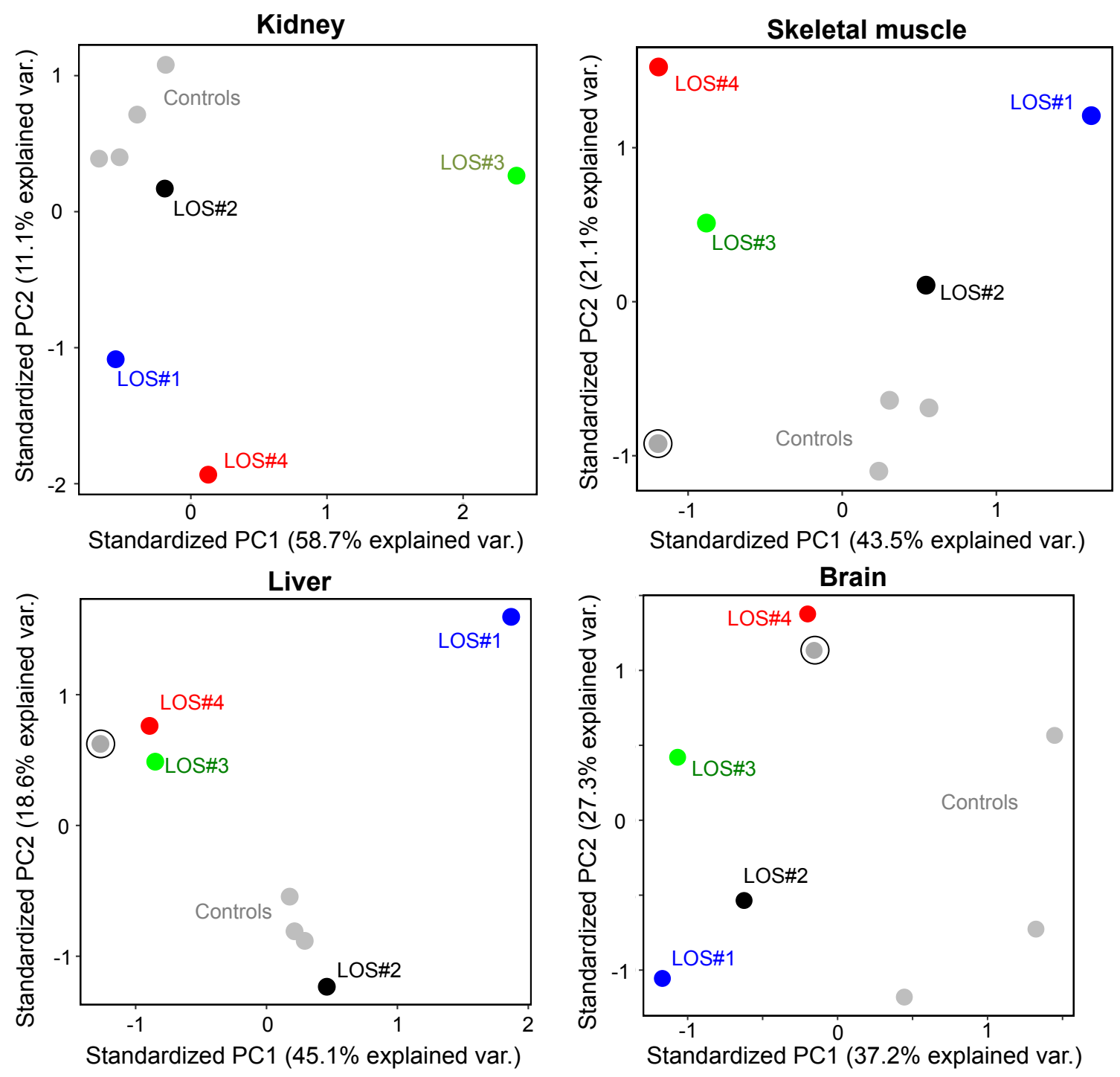

Figure S1. Principle component analyses using all expressed genes in kidney, skeletal muscle, liver, and brain. In skeletal muscle, liver, and brain, RNAseq library of Control \#2 (the grey dot circled with black) was sequenced with 50bp read length, while other fetuses were sequenced with $100 \mathrm{bp}$ read length. The read length differences may explain why Control \#2 fetus is not closely clustered with other control fetuses in these tissues. 


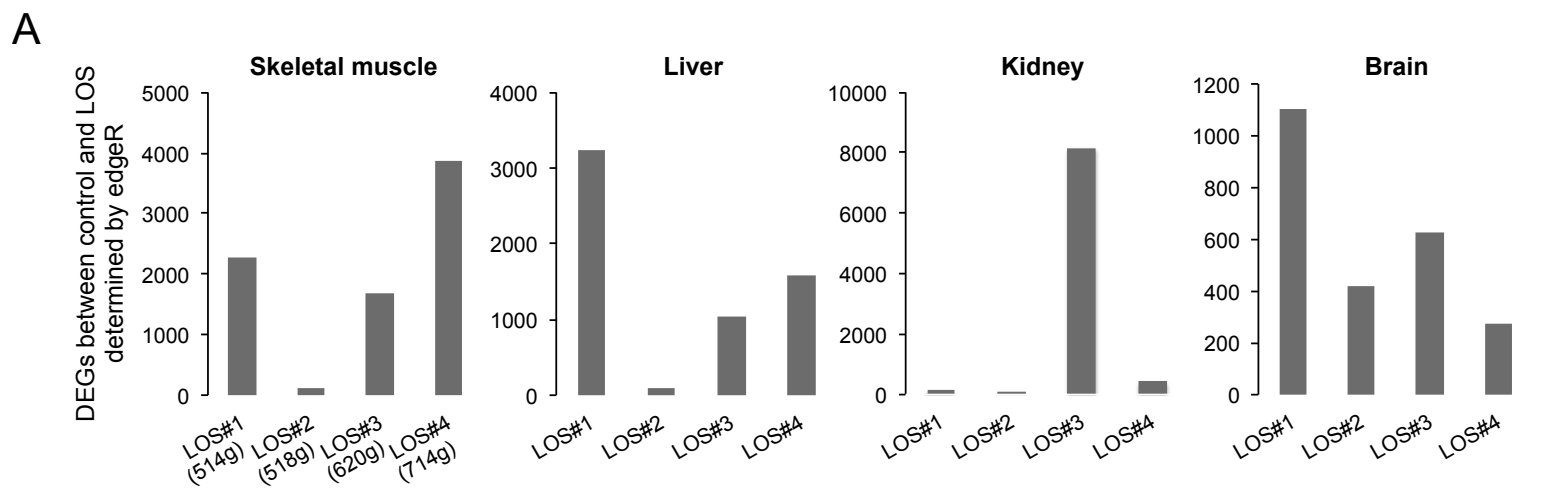

Panel $B$ and $C$ can be found in next page.

Figure S2. Identification and characterization of DEGs identified in the LOS fetuses. A).

Differentially expressed genes identified between each LOS fetus and the mean of the four controls. B). KEGG pathways enriched for DEGs in kidney and brain (FDR $<0.05$ ). C). KEGG pathways enriched for upregulated and downregulated genes in kidney and brain $(\mathrm{FDR}<0.05)$. 


\section{Figure S2}

B

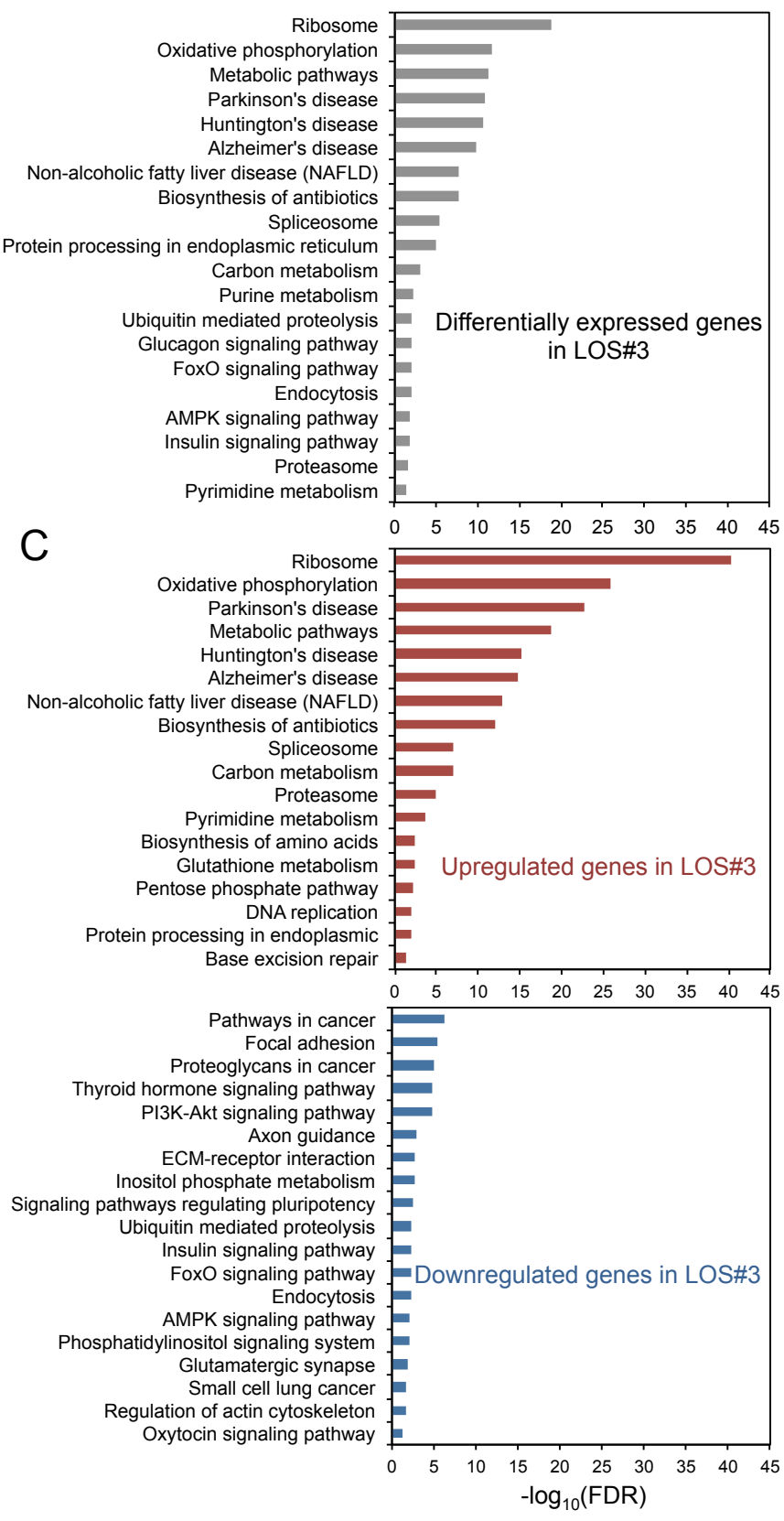

Brain

Differentially expressed genes in LOS\#1

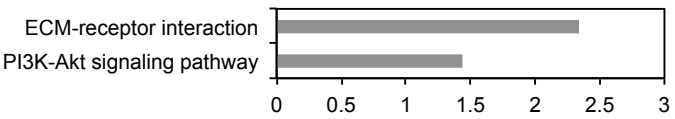

No significantly enriched KEGG pathways for upregulated genes in LOS \#1

Downregulated genes in LOS\#1

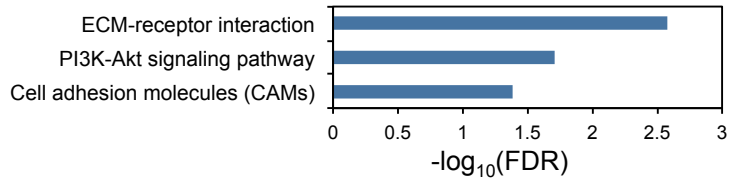


A

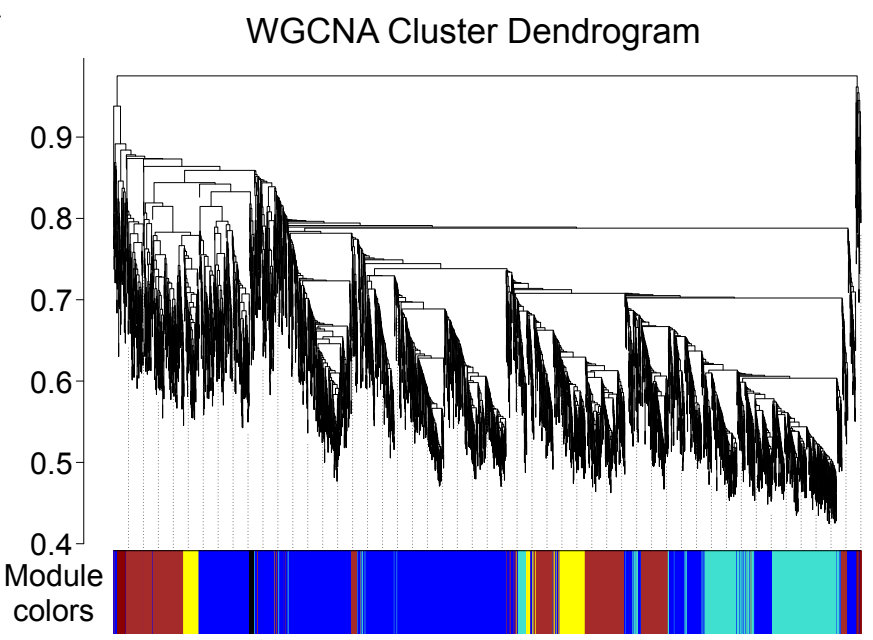

C

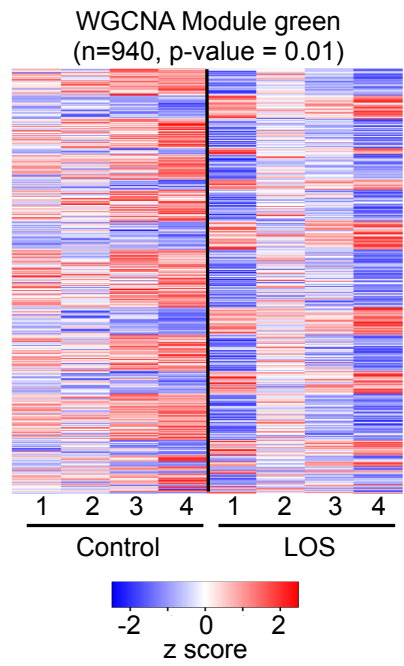

KEGG pathway analyses

- Spliceosome (FDR = 0.003)

Figure S3. WGCNA and KEGG pathway analyses in skeletal muscle. A). WGCNA cluster dendrogram on all control and LOS fetuses groups genes into 33 modules, namely, 33 co-expressed gene networks. B). Correlation of WGCNA modules and the bodyweight of the fetuses. Arrows indicate the three modules that show significant correlation with the bodyweight. C). Heat map profiling of the genes of module green. Module green includes genes that are mostly misregulated in LOS \#1 and \#4. D). Heat map profiling of genes that belong to the "Spliceosome" pathway. 


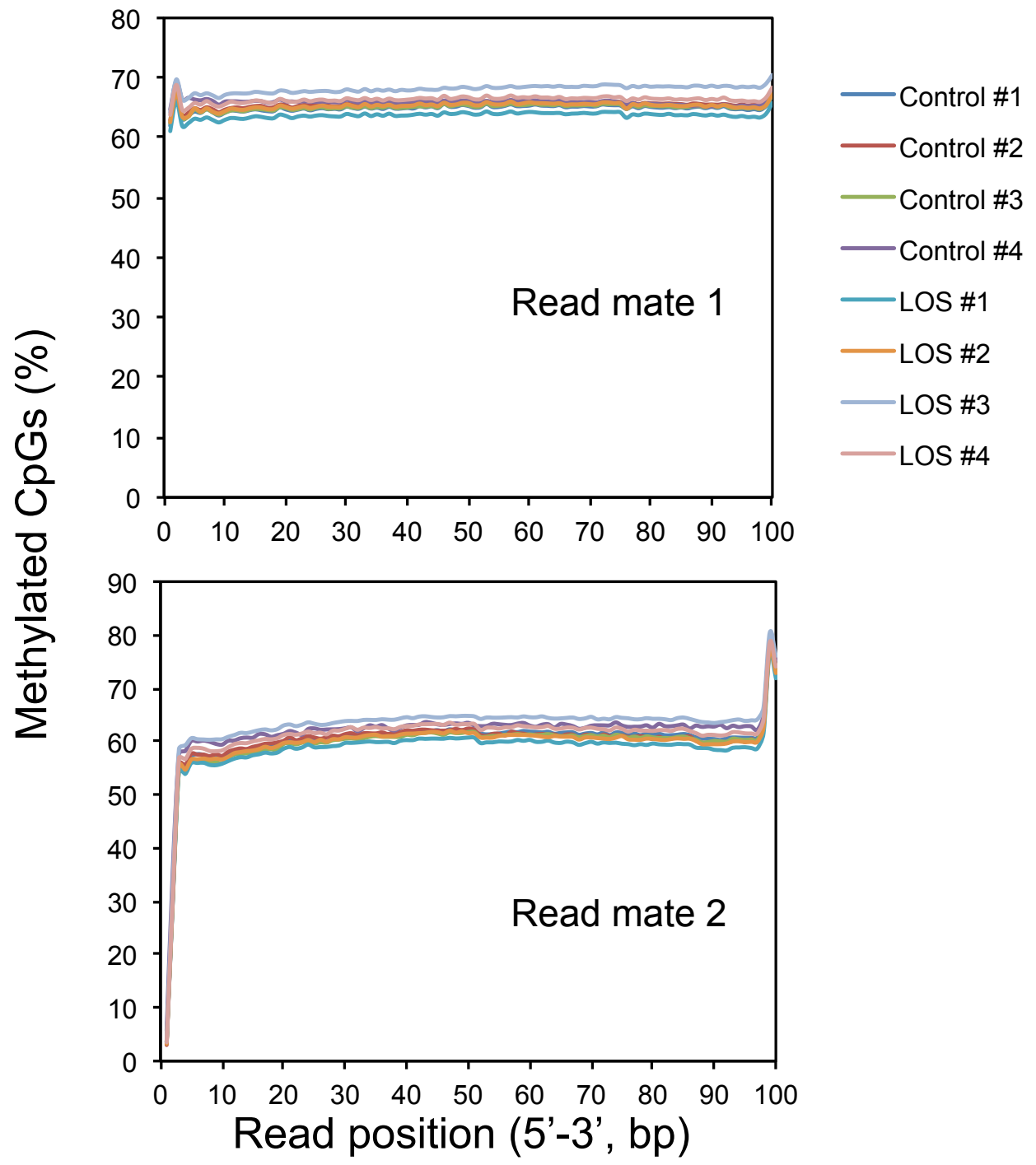

Figure S4. Mean CpG methylation level at each WGBS read position of the skeletal muscle from control and LOS fetuses. 
A

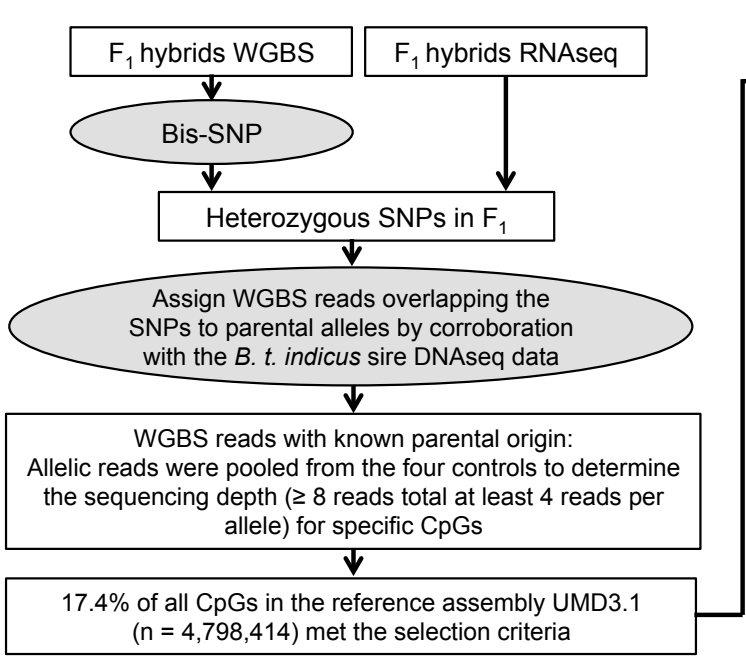

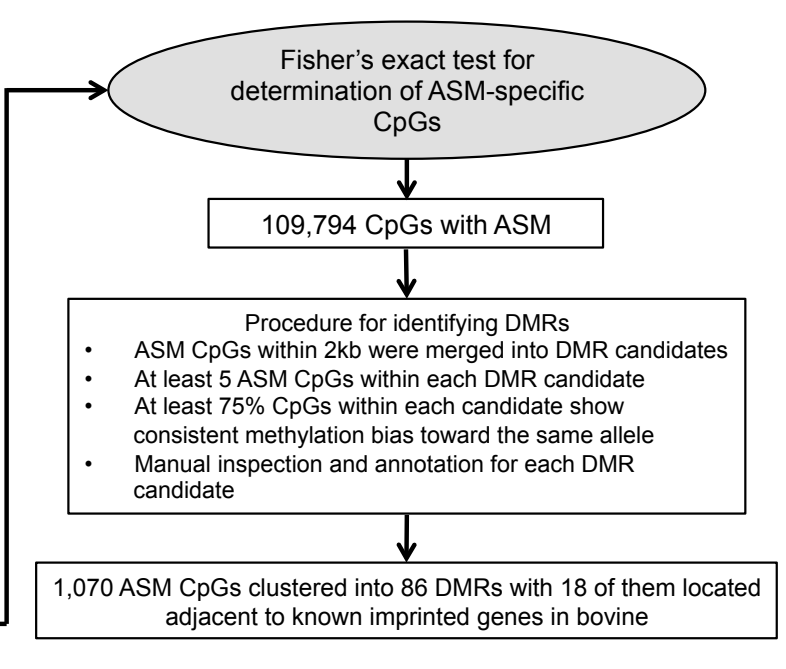

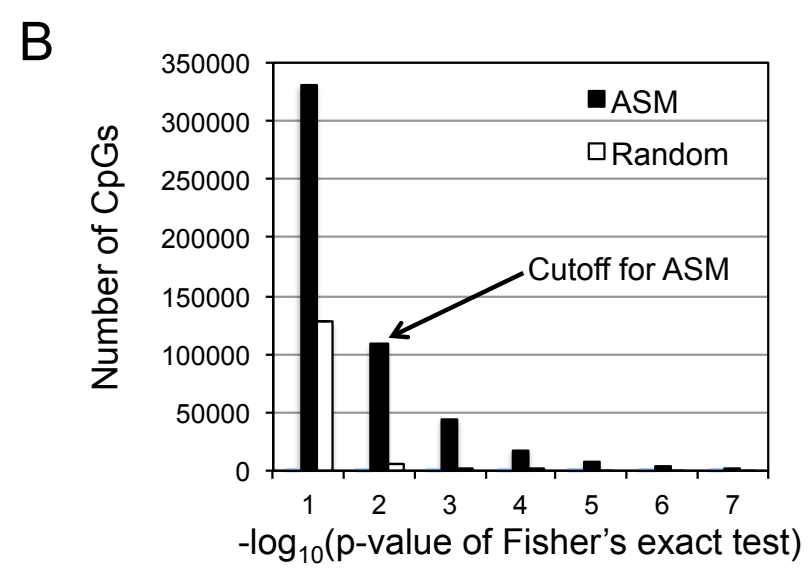

Figure S5. Pipeline used for the identification of ASM regions in skeletal muscle of control fetuses. A). Schematics pipeline. B). The number of ASM sites identified with various p-value cutoffs is plotted. The optimal cutoff choosed for identifying ASM sites is indicated. 
ASM regions not in imprinted loci

追 ASM regions in imprinted loci

A

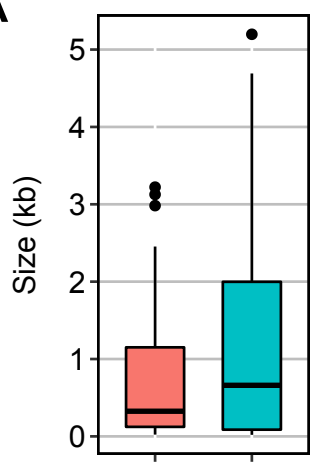

D

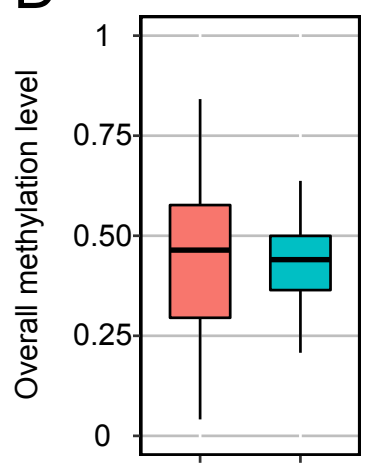

B

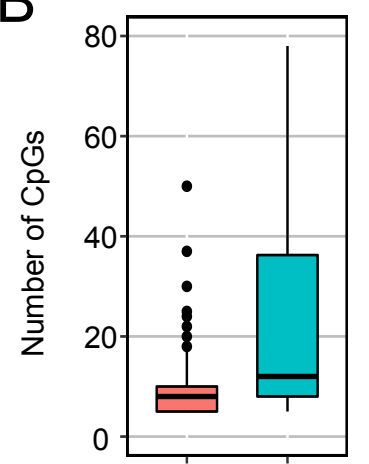

$\mathrm{E}$

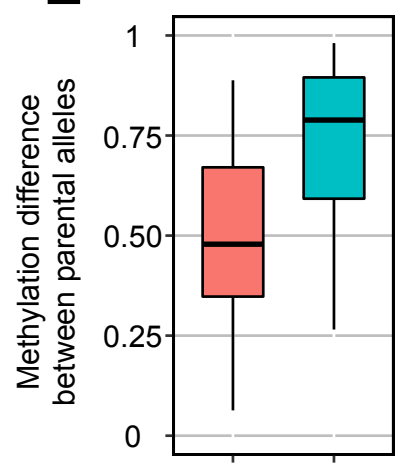

C

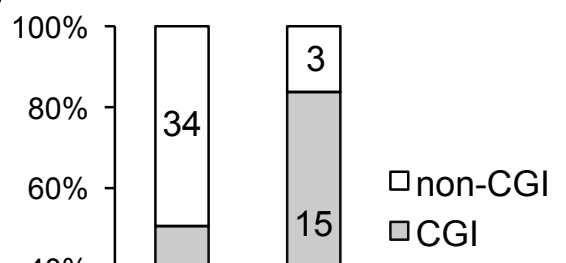

Figure S6. Characterization of the ASM regions identified in bovine fetal skeletal muscle. A) The size of the ASM regions. B) The number of CpGs in the ASM regions. Only CpGs identified with allele-specific DNA methylation were counted for each ASM region. C) Localization of the ASM regions in $\mathrm{CpG}$ islands. D) Overall methylation level for the ASM regions. E) Methylation differences between parental alleles for the ASM regions. CGI: CpG island. 
A

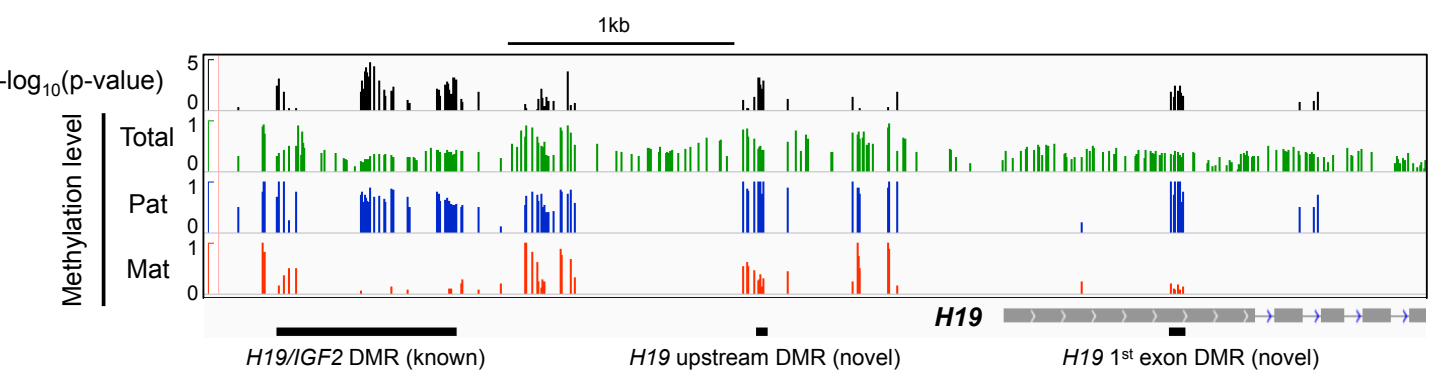

B

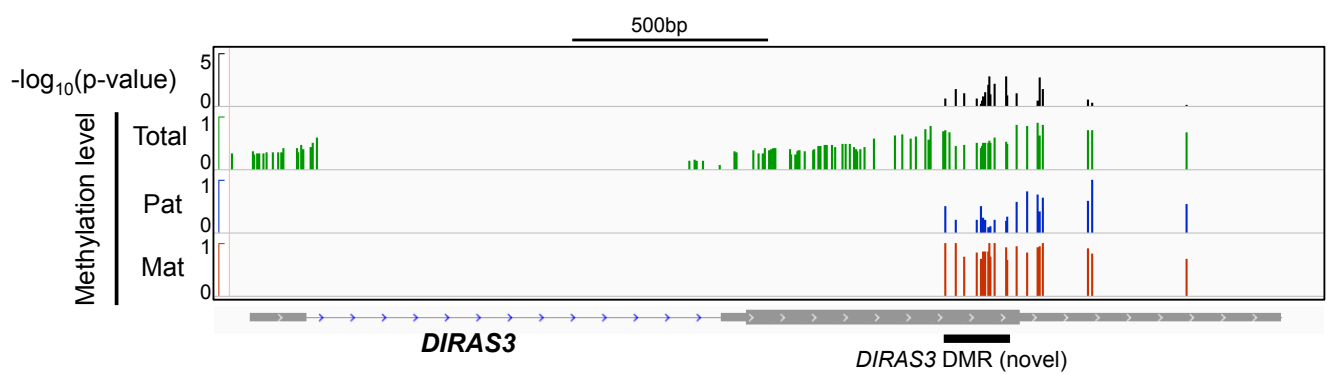

C

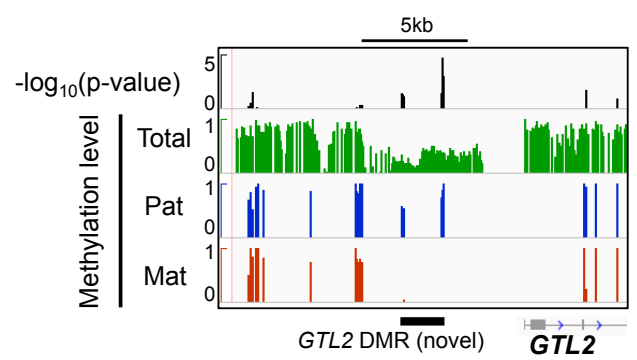

D

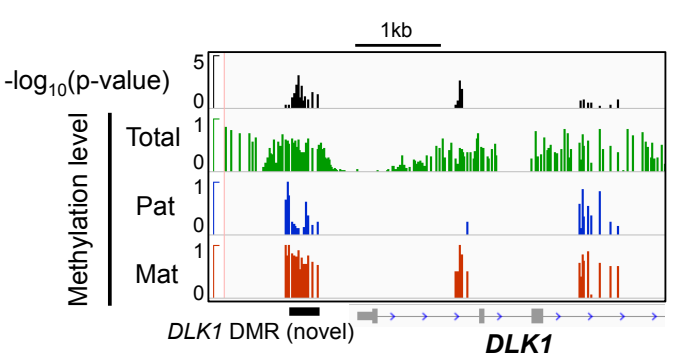

Figure S7. Examples of imprinted ASM loci. Genome browser views of the imprinted ASM loci: H19/IGF2, DIRAS3, and GTL2/DLK1. The $-\log _{10}$ (p-value) for ASM significance (black) and the $\mathrm{CpG}$ methylation levels for total (green), B. t. indicus allele (blue, paternal), and B. $t$. taurus allele (red, maternal) are also shown. Each bar represents a single $\mathrm{CpG}$ site. Mat: maternal allele; Pat: paternal allele. 
A

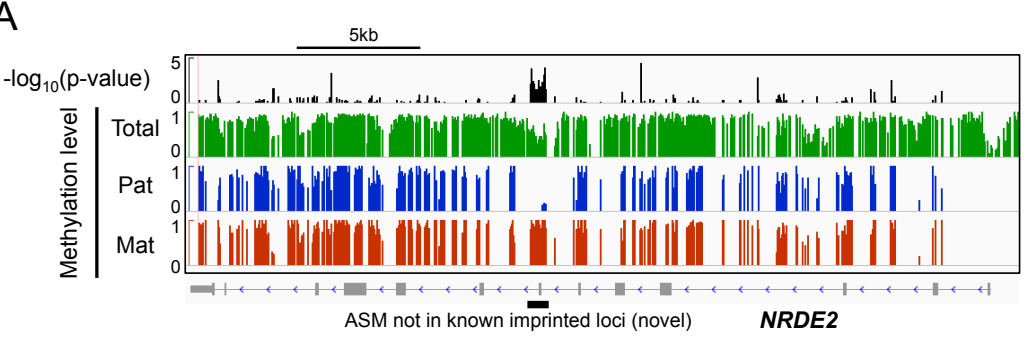

B

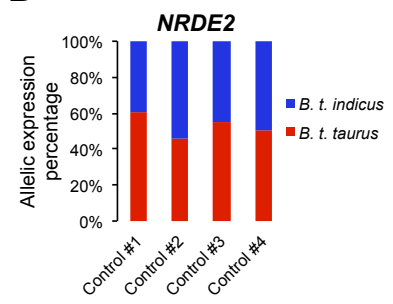

C

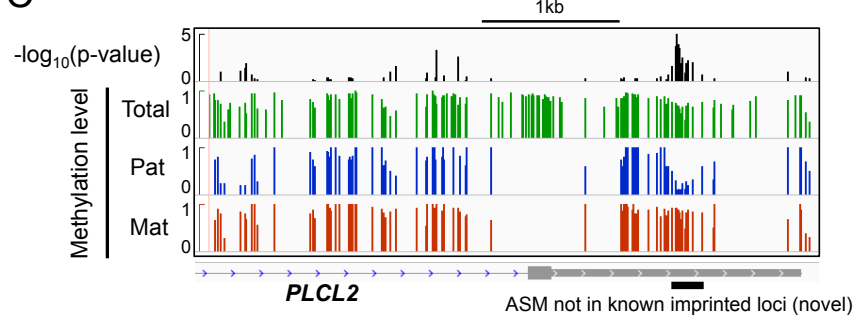

D

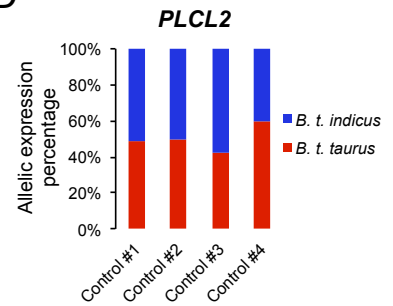

$\mathrm{F}$
$E$

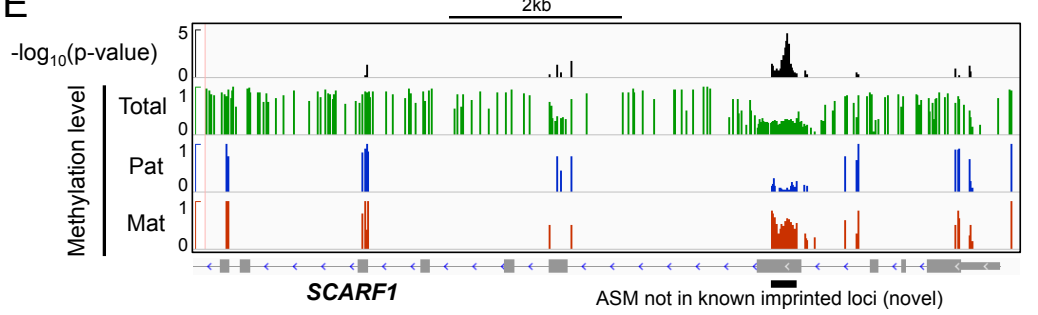

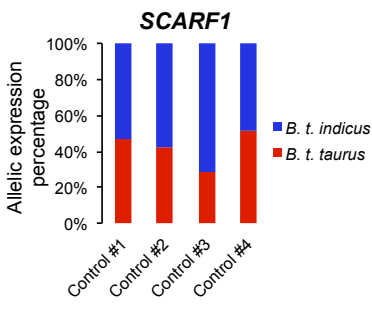

Figure S8. Examples of ASM regions at non-imprinted loci. A), C), and E). Genome browser views of the non-imprinted ASM loci: NRDE2, PLCL2, and SCARF1. The $-\log _{10}(\mathrm{p}-$ value) for ASM significance (black) and the $\mathrm{CpG}$ methylation levels for total (green), B. $t$. indicus allele (blue, paternal), and B. t. taurus allele (red, maternal) are also shown. Each bar represents a single $\mathrm{CpG}$ site. B), D), and F). Allelic expression percentage in skeletal muscle of the control fetuses. Mat: maternal allele; Pat: paternal allele. 
A

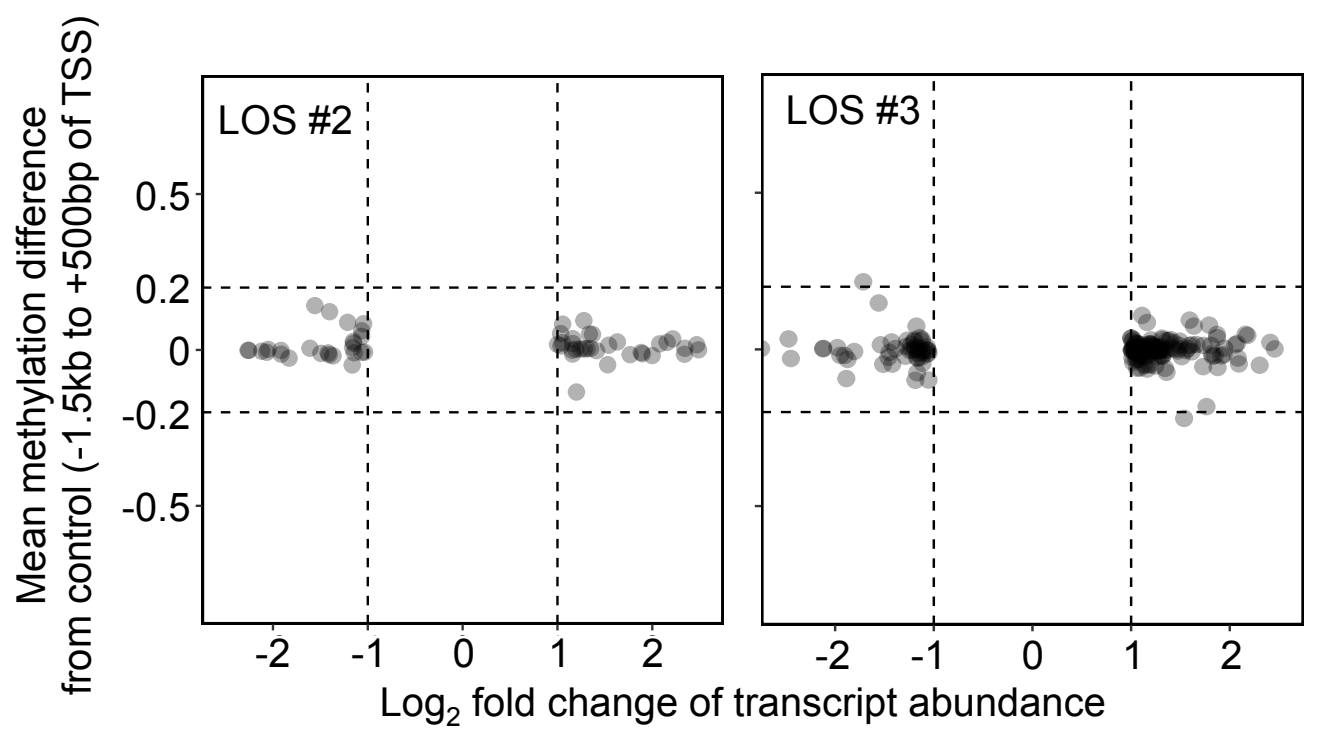

B

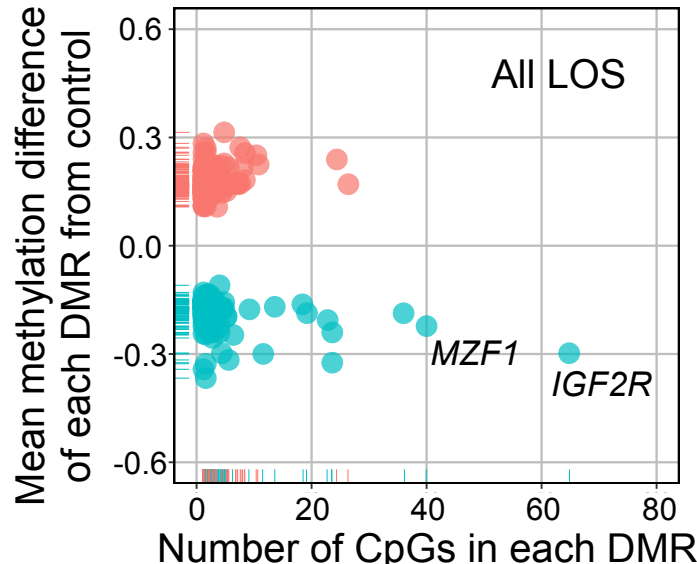

Hypermethylated DMRs

Hypomethylated DMRs

Figure S9. A). Mean methylation level differences of the DEGs between controls and LOS \#2 and \#3 at the TSS region (-1.5kb to +500bp). Only DEGs that have at least 2 -fold change are shown here. B). Number of $\mathrm{CpGs}$ and the mean methylation difference of each DMR identified when comparing all LOS fetuses to the controls. 
Table S1. Summary of the reads alignment and bisulfite conversion rate for each WGBS library

\begin{tabular}{|c|c|c|c|c|c|c|c|c|}
\hline Libraries & Bodyweight & $\begin{array}{c}\text { Gestational } \\
\text { day }\end{array}$ & Raw reads & Aligned reads & $\begin{array}{l}\text { Aligned } \\
\text { reads (\%) }\end{array}$ & $\begin{array}{c}\text { Uniquely } \\
\text { aligned reads }\end{array}$ & $\begin{array}{c}\text { Uniquely } \\
\text { aligned reads } \\
(\%)\end{array}$ & $\begin{array}{c}\text { Bisulfite } \\
\text { conversion rate } \\
(\%)\end{array}$ \\
\hline Control \#1 & $392 g$ & Day 104 & $396,328,924$ & $333,030,504$ & 84.0 & $267,417,844$ & 67.5 & 99.73 \\
\hline Control \#2 & $406 \mathrm{~g}$ & Day 105 & $428,296,878$ & $331,841,555$ & 77.5 & $266,934,993$ & 62.3 & 99.77 \\
\hline Control \#3 & $408 g$ & Day 106 & $418,901,079$ & $336,561,860$ & 80.3 & $268,410,454$ & 64.1 & 99.75 \\
\hline Control \#4 & $416 \mathrm{~g}$ & Day 106 & $355,810,944$ & $289,745,138$ & 81.4 & $229,015,740$ & 64.4 & 99.56 \\
\hline LOS \#1 & $514 \mathrm{~g}$ & Day 106 & $424,025,153$ & $338,241,972$ & 79.8 & $268,667,106$ & 63.4 & 99.77 \\
\hline LOS \#2 & $518 \mathrm{~g}$ & Day 105 & $431,258,953$ & $333,939,082$ & 77.4 & $260,337,387$ & 60.4 & 99.75 \\
\hline LOS \#3 & $620 \mathrm{~g}$ & Day 106 & $397,474,455$ & $320,530,155$ & 80.6 & $254,012,990$ & 63.9 & 96.48 \\
\hline LOS \#4 & $714 \mathrm{~g}$ & Day 105 & $421,457,025$ & $338,500,780$ & 80.3 & $269,718,690$ & 64.0 & 99.74 \\
\hline
\end{tabular}

Table S2. Reads coverage at CpG sites

\begin{tabular}{cccccc}
\hline Sample & $\geq 1$ read $\left(\%^{*}\right)$ & $\geq 3$ reads $\left(\%^{*}\right)$ & $\geq 5$ reads $\left(\%^{*}\right)$ & $\geq 8$ reads $\left(\%^{*}\right)$ & $\geq 10$ reads $\left(\%^{*}\right)$ \\
\hline Control \#1 & $25,422,282(92.3)$ & $24,523,876(89.0)$ & $22,835,084(82.9)$ & $18,144,557(65.9)$ & $13,953,964(50.7)$ \\
Control \#2 & $25,380,860(92.2)$ & $24,233,110(88.0)$ & $22,007,331(79.9)$ & $16,221,532(58.9)$ & $11,560,309(42.0)$ \\
Control \#3 & $25,420,791(92.3)$ & $24,414,185(88.6)$ & $22,484,754(81.6)$ & $17,326,183(62.9)$ & $12,984,193(47.1)$ \\
Control \#4 & $25,260,324(91.7)$ & $23,354,088(84.8)$ & $19,582,672(71.1)$ & $11,582,224(42.0)$ & $6,717,916(24.4)$ \\
LOS \#1 & $25,431,432(92.3)$ & $24,491,532(88.9)$ & $22,732,952(82.5)$ & $17,931,727(65.1)$ & $13,732,790(49.9)$ \\
LOS \#2 & $25,427,128(92.3)$ & $24,505,589(89.0)$ & $22,772,178(82.7)$ & $18,057,629(65.6)$ & $13,958,856(50.7)$ \\
LOS \#3 & $25,426,184(92.3)$ & $24,550,790(89.1)$ & $22,958,659(83.4)$ & $18,522,677(67.3)$ & $14,501,482(52.7)$ \\
LOS \#4 & $25,416,233(92.3)$ & $24,368,611(88.5)$ & $22,334,119(81.1)$ & $16,895,545(61.3)$ & $12,340,998(44.8)$ \\
\hline
\end{tabular}

*Percentage of CpGs in the reference assembly UMD3.1 covered by at least $1,3,5,8$, and 10 reads. 


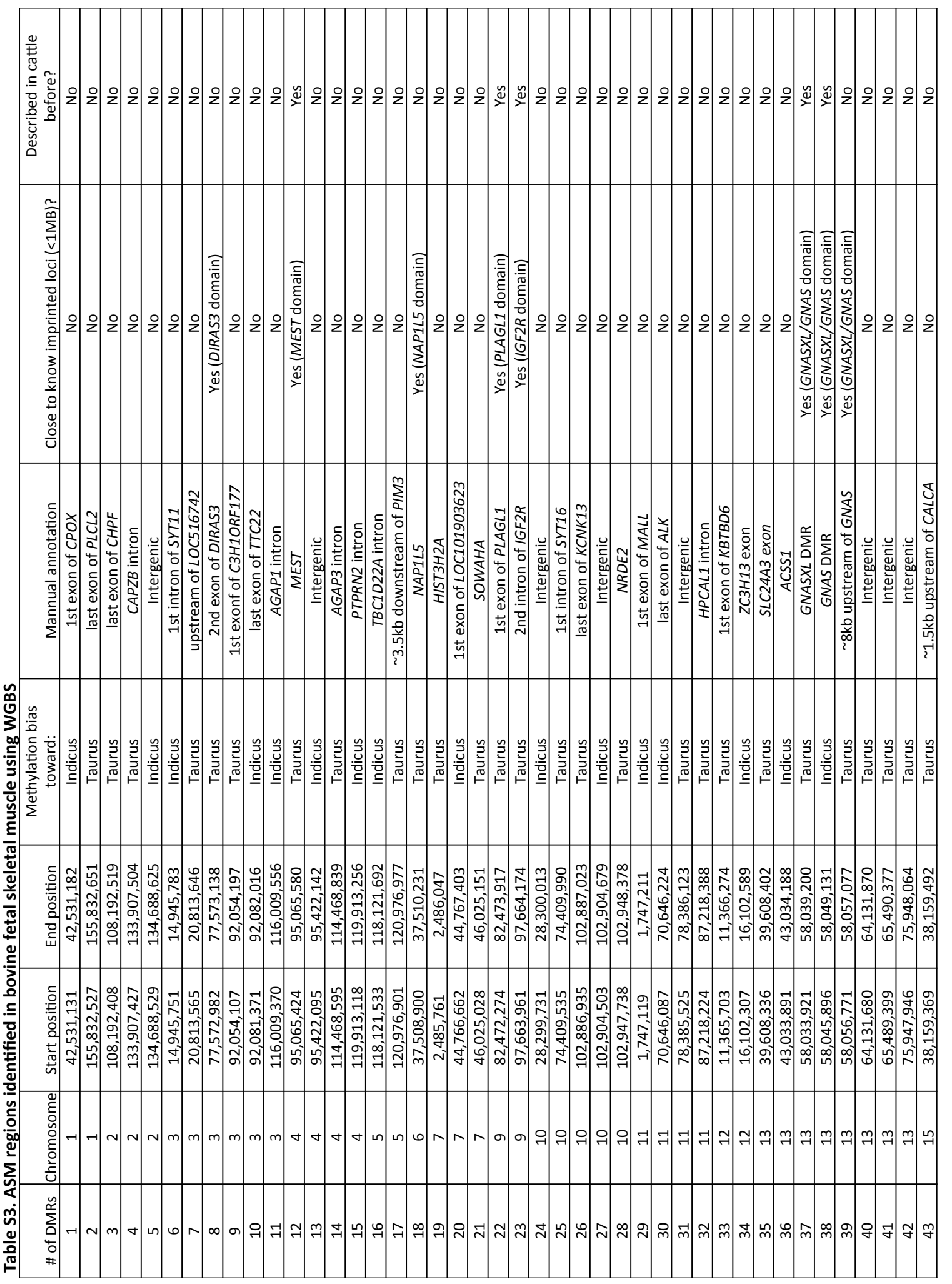




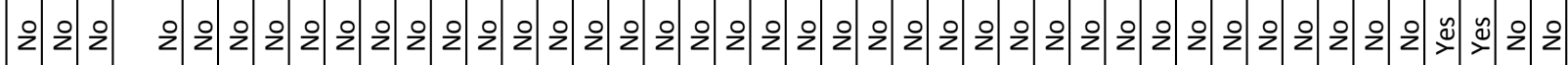

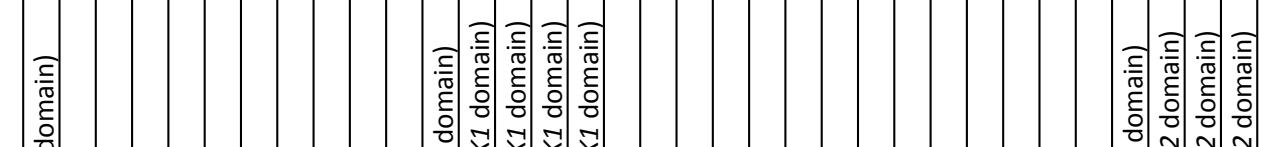

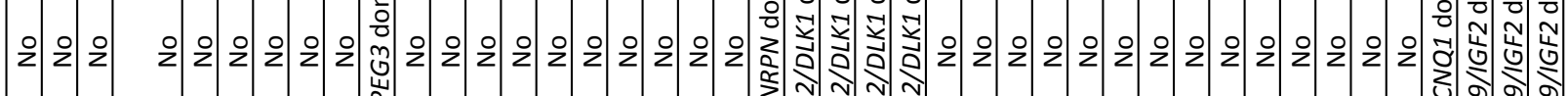

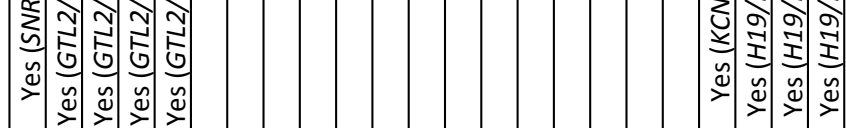

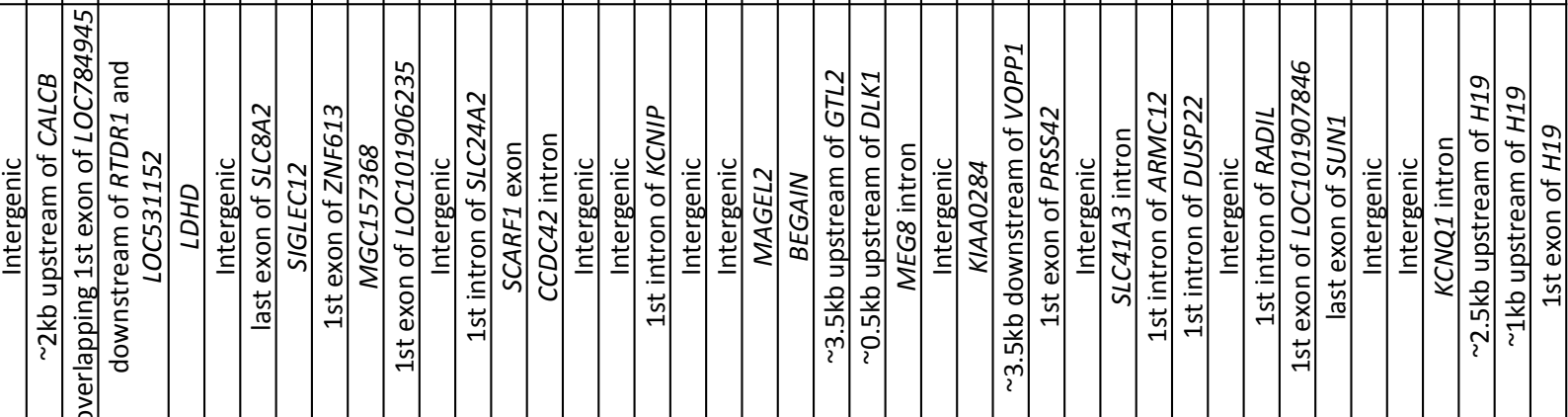

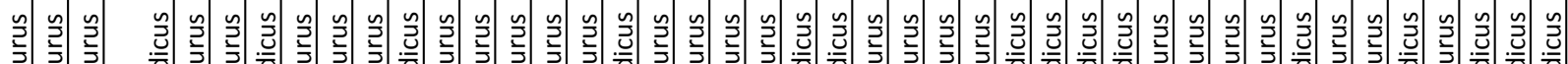
党

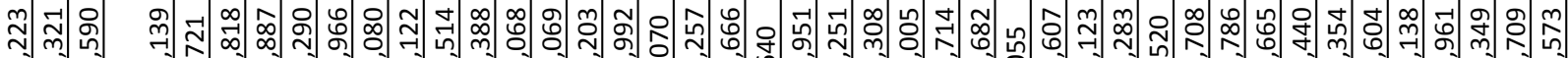

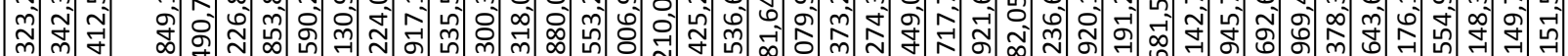

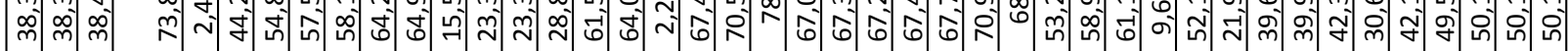

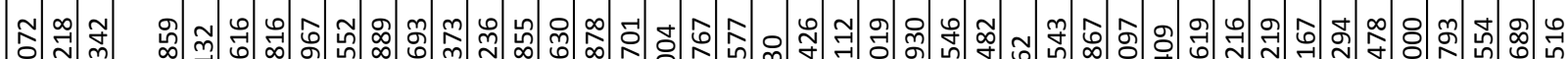

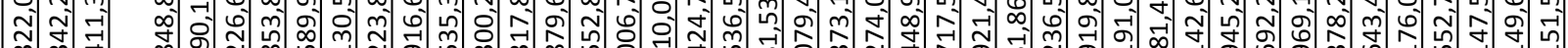
$m$
$\infty$
$\infty$

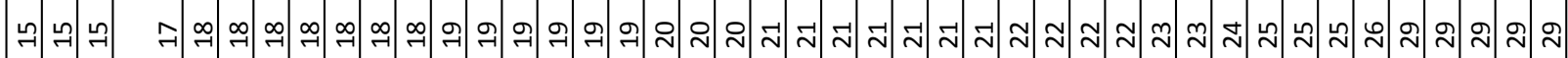

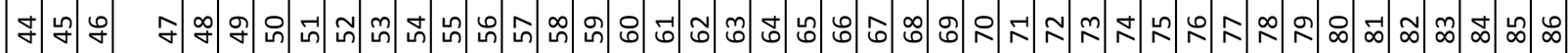




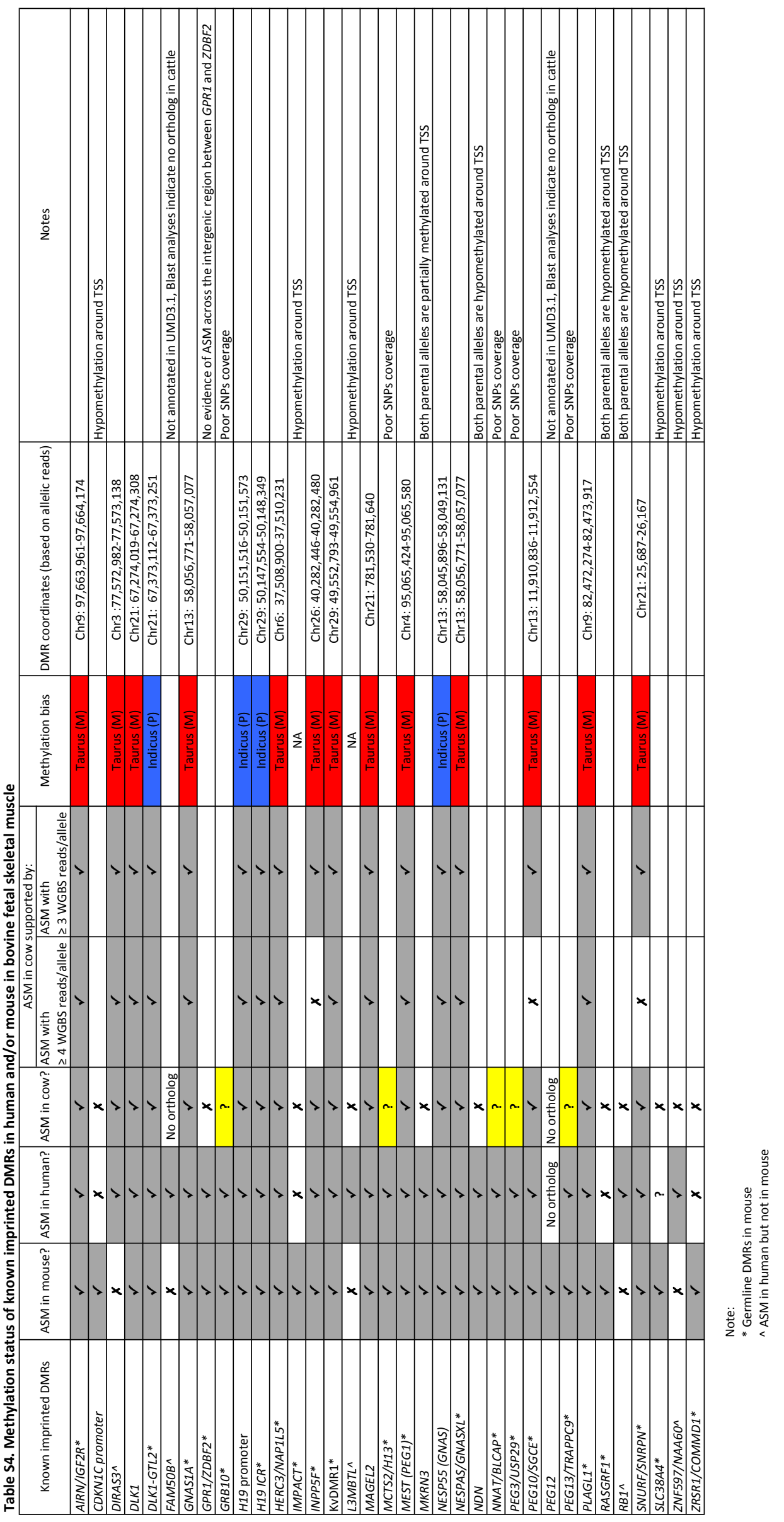




\section{Chapter 4 Global assessment of imprinted gene expression in the bovine conceptus by next generation sequencing}

\subsection{Abstract}

Genomic imprinting is an epigenetic mechanism that leads to parental-allelespecific gene expression. Approximately 150 imprinted genes have been identified in humans and mice but less than 30 have been described as imprinted in cattle. For the purpose of de novo identification of imprinted genes in bovine, we determined global monoallelic gene expression in brain, skeletal muscle, liver, kidney and placenta of day $\sim 105$ Bos taurus indicus $\times$ Bos taurus taurus $\mathrm{F}_{1}$ conceptuses using RNA sequencing. To accomplish this, we developed a bioinformatics pipeline to identify parent-specific SNP alleles after filtering A-to-I RNA editing sites. We identified 53 genes subject to monoallelic expression. Twenty three are genes known to be imprinted in the cow and an additional 7 have previously been characterized as imprinted in human and/or mouse that have not been reported as imprinted in cattle. Of the remaining 23 genes, we found that 10 are uncharacterized or unannotated transcripts located in known imprinted clusters, whereas, the other 13 genes are distributed throughout the bovine genome and are not close to any known imprinted clusters. To exclude potential cis-eQTL effects on allele expression, we corroborated the parental specificity of monoallelic expression in day 86 Bos taurus taurus $\times$ Bos taurus taurus conceptuses and identified eight novel bovine imprinted genes. Further, we identified 671 candidate A-to-I RNA editing sites and describe random $\mathrm{X}$-inactivation in day 15 bovine extraembryonic membranes. Our results expand the imprinted gene list in bovine and demonstrate that monoallelic gene 
expression can be the result of cis-eQTL effects.

\subsection{Introduction}

In mammals, approximately 150 genes have been shown to be expressed in a parental-allele-specific manner (Bartolomei and Ferguson-Smith, 2011). These genes, collectively known as imprinted genes, are essential for normal embryonic growth, placental function, and postnatal behavior (Peters, 2014) and their misregulation has been linked to several developmental syndromes and types of cancers (Kalish et al., 2014; Lim and Maher, 2010). Most imprinted genes are organized in clusters and are regulated by cluster-specific DNA elements termed imprinting control regions (ICR; Bartolomei and Ferguson-Smith, 2011). ICRs are marked with DNA methylation and histone posttranslational modifications in a sex-specific manner during gametogenesis (Bartolomei and Ferguson-Smith, 2011). These epigenetic signatures are maintained during development and serve to differentiate the parental alleles in somatic cells, when both genomes are found within the same nucleus (Bartolomei and Ferguson-Smith, 2011). Since the discovery of the first three imprinted genes in 1991 (Barlow et al., 1991; Bartolomei et al., 1991; DeChiara et al., 1991), more than 100 imprinted genes have been identified in humans and mice (http://igc.otago.ac.nz; http://www.har.mrc.ac.uk/research/genomic_imprinting/). However, the list of imprinted genes is not yet comprehensive as their expression can vary by tissue and stage of development (Bartolomei and Ferguson-Smith, 2011; Peters, 2014). 
Many fewer imprinted genes $(n=34)$ have been reported in domestic farm animal species (Tian, 2014). In addition, most imprinted genes in cow, pig, sheep, and rabbit have been characterized in studies designed to corroborate the allele-specific expression of genes previously described as imprinted in human and/or mouse (Bischoff et al., 2009; Chen et al., 2015; Chen et al., 2013; Okamura et al., 2005; Robbins et al., 2012; Young et al., 2003; Zaitoun and Khatib, 2006). As in human and mouse, allele specificity in monoallelic expression of these genes is a requirement for proper embryonic and fetal growth; loss-of-imprinting (LOI) has been associated with abnormal development of the conceptus (Chen et al., 2013; Farin et al., 2006; Tian, 2014; Young et al., 2001). One such example is large offspring syndrome (LOS) in bovine and ovine (Young et al., 1998), a fetal overgrowth condition that is phenotypically and epigenetically similar to the loss-of-imprinting condition Beckwith-Wiedemann syndrome in human (Chen et al., 2013). In a previous study (Chen et al., 2015), we analyzed a group of genes known to have imprinted expression in human and/or mouse and showed loss of monoallelic expression and differential transcript abundance of multiple imprinted genes in LOS. We also found that some of the genes (e.g., COPG2, IMPACT, HTR2A) do not express in the expected monoallelic fashion but rather are biallelically expressed in the bovine conceptus. The species specificity of genomic imprinting suggests that certain genes may only be imprinted in cattle; therefore, de novo identification of imprinted genes using genome-wide scale approaches is required in order to achieve a more thorough understanding of this epigenetic mechanism and its role in fetal development of this economically important species. 
Advances in high throughput sequencing technology provide an unprecedented opportunity for the discovery of imprinted genes at the transcriptome level and has led to the discovery of additional imprinted genes in mice and humans (Wang and Clark, 2014). In mice, transcriptome wide identification of imprinted genes relies on RNA sequencing (from here on referred to as RNAseq) of $F_{1}$ offspring resulting from reciprocal crosses of distantly related inbred mice to identify parental-allele-specific gene expression (Babak et al., 2008; Wang et al., 2008). While this approach has been successful, bioinformatics analyses alone can suffer from a high false positive rate due to the potential technical artifacts of RNAseq (DeVeale et al., 2012). Thus, the use of next generation sequencing to identify imprinted genes requires independent validation through traditional methods (i.e., Sanger sequencing). In human, systemic characterization of genomic imprinting using RNAseq has recently been performed in 46 tissues from 178 adults (Baran et al., 2015). This study highlights the widespread tissue-specificity of genomic imprinting as well as the species-specificity of imprinting (some genes imprinted in human are not imprinted in the mouse) (Baran et al., 2015).

Beyond genomic imprinting, $\mathrm{X}$ chromosome inactivation $(\mathrm{XCI})$ is another epigenetic phenomenon that can regulate gene dosage by silencing one of the parental alleles in females (Lee and Bartolomei, 2013). Contrary to parental-origin-dependent silencing of imprinted genes, dosage compensation of X-linked genes in most organs is achieved by randomly silencing one of the X chromosomes (Lyon, 1961). However, in extra-embryonic tissues of mouse and rat, $\mathrm{XCI}$ is imprinted and it is always the paternal X chromosome that is inactivated (Huynh and Lee, 2001; Wake et al., 1976). It has not been resolved whether XCI is imprinted or random in the human placenta (Lee and 
Bartolomei, 2013) and, in cattle, a study based on the allele-specific expression of the Xlinked gene $M A O A$ indicates that $\mathrm{XCI}$ is imprinted in the placenta with preferential silencing of the paternal X chromosome (Xue et al., 2002).

In this study, we determined global allele-specific gene expression in day $\sim 105$ Bos taurus indicus $($ B. t. indicus $) \times$ Bos taurus taurus $\left(\right.$ B. t. taurus) $\mathrm{F}_{1}$ conceptuses (gestation length $\sim 280$ days) using RNAseq. We developed an analysis pipeline to identify monoallelically expressed genes by resolving several technical challenges such as correction of allelic mapping bias of RNAseq reads and filtering potential RNA editing sites in RNAseq data and then validated our findings by Sanger sequencing. Further, we corroborated the parental specificity of monoallelic expression in day 86 B. t. taurus $\times$ B. t. taurus (Holstein breed) conceptuses to exclude potential cis-eQTL effects on allele expression. Finally, we describe random X-inactivation in day 15 bovine extraembryonic membranes.

\subsection{Results}

Pipeline for identification of monoallelically expressed genes in bovine using RNAseq

The identification of imprinted gene expression requires the use of DNA polymorphisms to identify parental origin of the alleles in an $\mathrm{F}_{1}$ individual. For bovids, it is not always possible to know the identity of the parental alleles, for example, if those individuals were conceived with oocytes obtained from abattoir ovaries. This fact, together with the fact that cattle are not inbred, makes the global identification of genes 
subject to allele-specific expression using RNAseq a challenging task. Another challenge when working in bovine is encountered when aligning RNAseq reads to the publically available reference genome (i.e., UMD3.1) because only one B. t. taurus female (i.e., Hereford breed) is documented with only one of her alleles recorded. In the present study, we developed a bioinformatics pipeline (Fig. 1) to identify candidate imprinted genes in cattle using RNAseq data from four gestation day $\sim 105$ female bovine conceptuses (fetus and placenta) generated from the breeding of one Nelore bull (ABS CSS MR N OB 425/1 677344 29NE0001 97155 [i.e., B. t. indicus]) to four Holstein breed (B. t. taurus) females of unknown genetics (Chen et al., 2013). We utilized this breeding scheme, which involves fertile mating between subspecies of cattle that diverged $\sim 620,000$ years ago (MacHugh et al., 1997), to increase the likelihood of finding informative polymorphisms with which to ascribe parental origin of the nucleic acid being studied.

First, the sire's DNA was subjected to next generation sequencing (DNAseq) to identify all single nucleotide polymorphisms (SNPs) between his genome and the B. $t$. taurus reference genome UMD3.1 build. In total, 347.3 million $2 \times 100$ bp paired-end reads ( 20X coverage of the bovine reference genome) were obtained (Fig. S1A) and used to genotype the B. t. indicus sire. We used Genome Analysis Toolkit (GATK; McKenna et al., 2010) and SAMtools (Li, 2011) pipelines for SNP calling and only SNPs identified by both pipelines (i.e., 93.11\%; Fig. 1 and Fig. S1B and C) were used to edit the reference genome to generate a pseudo B. t. indicus genome. Aligning RNAseq reads to a non-identical reference genome has been shown to introduce alignment bias (Degner et al., 2009; Stevenson et al., 2013) so we combined the reference genome and the pseudo 


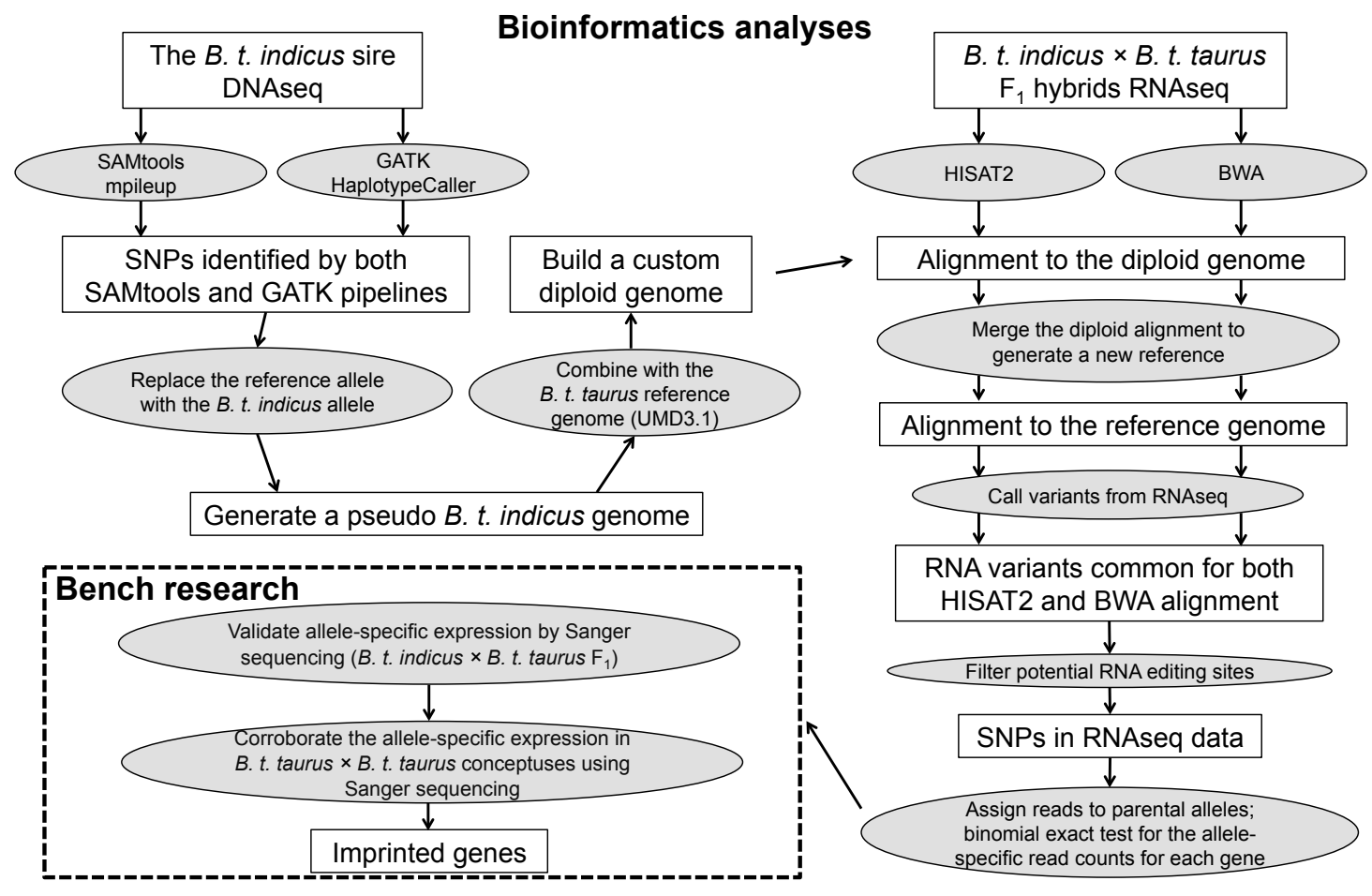

Figure 1. Summary of the pipelines used in this study. Top left and right of figure show the bioinformatics methods used to identify monoallelically expressed genes in day $\sim 105$ Bos taurus indicus $($ B. $t$. indicus $) \times$ Bos taurus taurus $\left(\right.$ B. t. taurus) $\mathrm{F}_{1}$ conceptuses. Bottom left shows the pipeline used to corroborate the findings of the bioinformatics analyses. SNP $=$ single nucleotide polymorphism. 
B. $t$. indicus genome to create a custom diploid genome. RNAseq reads from the $B . t$. indicus $\times B$. t. taurus $\mathrm{F}_{1}$ conceptuses were mapped to the diploid genome using both the HISAT2 (Kim et al., 2015) and BWA (Li and Durbin, 2009) pipelines and only variants identified by both methods were retained for further analyses (Fig. 1) to minimize the potential effects of false positives.

Genotyping the day $\sim 105$ B.t. indicus $\times$ B. t. taurus $F_{1}$ conceptuses using RNAseq

RNAseq data were obtained from brain, kidney, liver, skeletal muscle and placenta of the four conceptuses (i.e., Control \#1, \#2, \#3, and \#4; Table S1) and were aligned to the custom B. t. indicus/B. t. taurus diploid genome. We adapted the SNPiR pipeline (Piskol et al., 2013) to identify SNPs using all RNAseq reads from each conceptus. To minimize false positive SNP calls, in addition to the default pipeline (BWA; Li and Durbin, 2009; Piskol et al., 2013), we also mapped the reads using HISAT2 (Kim et al., 2015) and only retained variants common to both alignment strategies (Fig. S2A, Table S1). The identified RNA variants in the $\mathrm{F}_{1}$ conceptuses were compared to known bovine dbSNP entries (http://www.ncbi.nlm.nih.gov/SNP) and the identified B. t. indicus SNPs. We found that $\sim 98 \%$ of the RNA variants were matched to the bovine dbSNP entries and/or were supported by the $B$. $t$. indicus DNAseq reads and we refer to them as "genomic variants" (Fig. S2B). The genomic variants exhibited a $\sim 2.5$ transition/transversion ratio (see methods; Fig. S2C) while the remaining $\sim 2 \%$ RNA variants (Fig. S2B), hereinafter referred to as "RNA-DNA differences (RDDs)", exhibited 14-18 transition/transversion ratios and were enriched for $A>G$ and $T>C$ mismatches (Fig. S2D). 
Identification and validation of A-to-I RNA editing sites in B. t. indicus $\times$ B.t.

taurus conceptuses

We reasoned that some of the RDDs with $\mathrm{A}>\mathrm{G}$ and $\mathrm{T}>\mathrm{C}$ mismatches resulted from adenosine-to-inosine (A-to-I) RNA editing, a tissue specific post-transcriptional mechanism in which adenosine is converted to inosine in RNA duplexes by adenosine deaminase acting on RNAs (ADARs; Nishikura, 2010). A-to-I RNA editing is detected as $A>G$ mismatches in cDNA (Nishikura, 2010). To confirm the true identity of A-to-I RNA editing, we retrieved the variant information (i.e., read counts for both the reference and alternative alleles) in the RNAseq data of each tissue from each individual (Fig. 2A). Further, we required that each RDD have at least 10 reads coverage per tissue per conceptus to discriminate rare SNPs or sequencing errors from true RNA editing sites. For brain, liver, and skeletal muscle, only RDDs shared by Controls \#1, \#3, and \#4 were used to identify RNA editing sites since the RNAseq reads for these tissues from Control \#2 were sequenced at $50 \mathrm{bp}$ length instead of $100 \mathrm{bp}$ length for the other samples (Table S1). We identified 404, 275, 266, 208, and 180 RNA variants in brain, kidney, liver, skeletal muscle, and placenta, respectively (Fig. 2A). Of these, the majority ( $>72 \%)$ were $\mathrm{A}>\mathrm{G}$ mismatches (Fig. $2 \mathrm{~A}$ and $\mathrm{B}$ ) and we considered them to putatively represent A-to-I editing sites (Table S2; link to Table S2: http://www.tandfonline.com/doi/full/10.1080/15592294.2016.1184805).

We analyzed 7 predicated editing sites in four genes (i.e., COPA, SEC16A, $G R I K 2$, and $G A B R A 3$ ) using Sanger sequencing to confirm that the editing site did not represent a heterozygous SNP in DNA and also to corroborate the editing of the RNA 
A

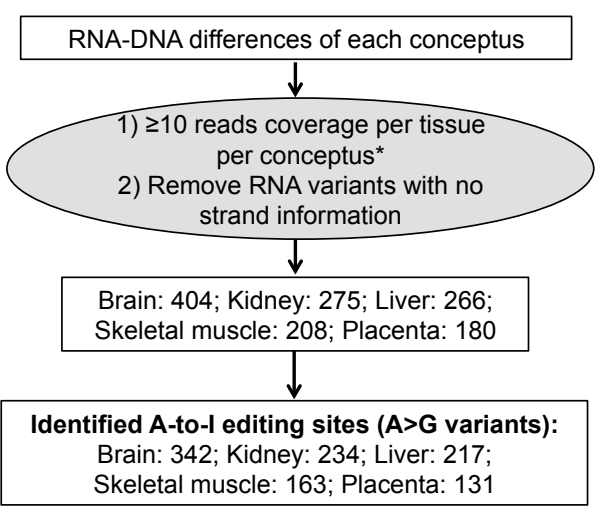

C

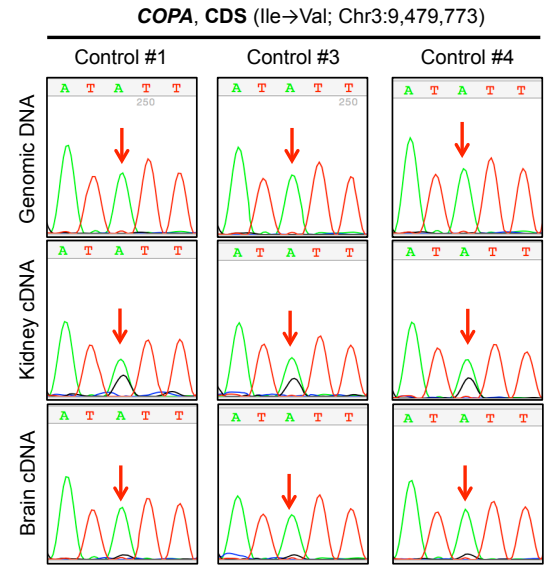

B

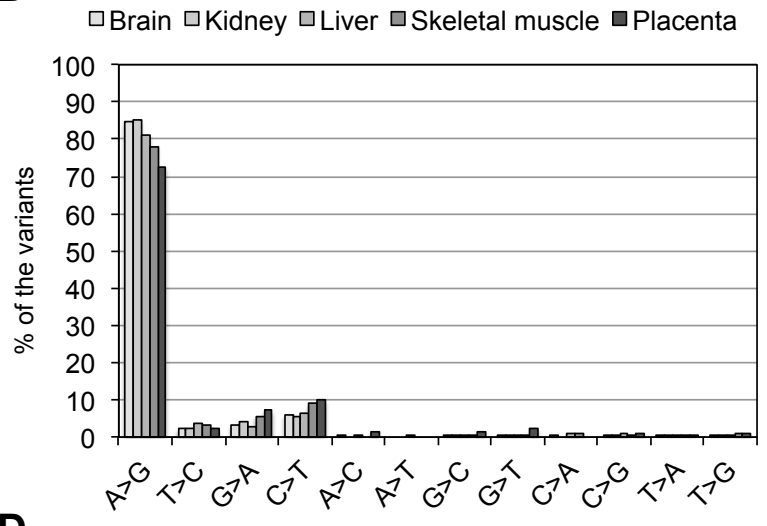

D

SEC16A, 3'UTR (Chr11: 103,938,967) SEC16A, 3'UTR (Chr11: 103,938,750)

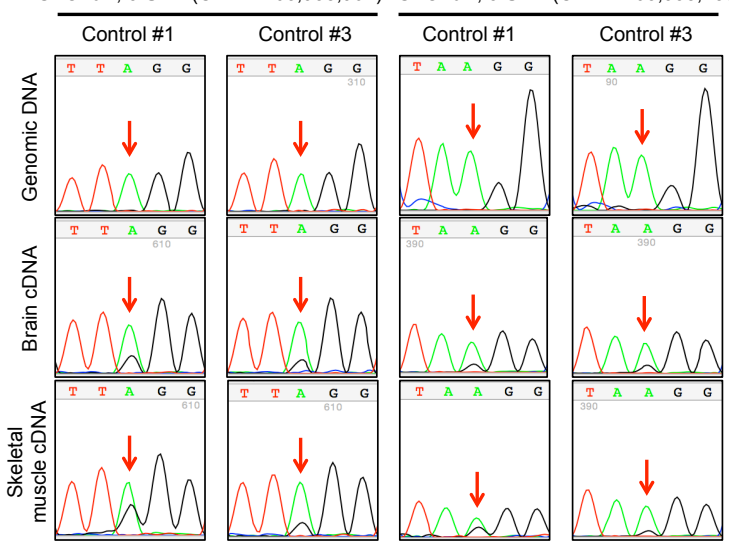

Figure 2. Identification of adenosine-to-inosine (A-to-I) RNA editing sites in day $\sim \mathbf{1 0 5}$

B. t. indicus $\times$ B t. taurus $\mathbf{F}_{1}$ conceptuses. A). Pipeline used to identify which RNA-DNA differences (RDDs) were potential A-to-I RNA editing sites. RDDs refer to RNA variants not found in the bovine dbSNP database nor in the $B$. $t$. indicus sire DNAseq reads. RNA variants were identified using all the RNAseq reads of each conceptus. Only RDDs with $\geq 10$ reads coverage per tissue per conceptus and had strand information of the transcript template were used to identify potential editing sites. *Note: for brain, liver, and skeletal muscle only RDDs shared by Control \#1, \#3, and \#4 were used to identify RNA editing sites since the RNAseq reads for those tissues in Control \#2 were sequenced at $50 \mathrm{bp}$ length instead of $100 \mathrm{bp}$ length for all other samples. B). Variant type distribution of the RDDs. "A $>G$ " variants were considered candidate A-to-I RNA editing sites (detailed information of these sites may be found in Table S2). C) and D). Validation of the identified A-to-I RNA editing sites by Sanger sequencing. Red arrows indicate the editing sites. Panel $\mathbf{C}$ shows an example of A-to-I editing in the coding sequence (CDS) of COPA, which can cause an amino acid change (Ile $\rightarrow \mathrm{Val}$ ) while panel $\mathbf{D}$ shows two editing sites in the 3' untranslated region (3'UTR) of SEC16A. Further verification of A-to-I RNA editing sites may be found in Figure $\mathrm{S} 3$. 
molecules (a second "G" peak in the sequencing chromatogram). We were able to validate 6 of 7 to be true A-to-I editing sites (Fig. 2C and D, Fig. S3).

\section{Characterization and species conservation of candidate A-to-I editing sites}

Analysis of the putative A-to-I editing events indicated that $~ 96 \%(643 / 671)$ of

these sites were within noncoding regions such as UTRs and introns and approximately two percent (15/671) were located in coding sequences (CDS; Fig. S4). This observation is consistent with findings in human and mouse that the majority of A-to-I RNA editing events occur in non-coding regions (Ramaswami et al., 2013). Of the sites located in CDS', 14 could cause an amino acid change (Table S2).

To determine whether the identified A-to-I RNA editing sites in bovine were conserved in human and/or mouse, we retrieved $80 \mathrm{bp}$ of flanking sequences (40 bp upstream and $40 \mathrm{bp}$ downstream; Pinto et al., 2014) and aligned the 81 nucleotides to the human and mouse genomes using BLAST . A valid BLAST hit with $>86 \%$ identity was required to ascribe a site as being conserved between species (Pinto et al., 2014). With this criterion, 20 of the sites were predicted to be conserved between bovine and human and/or mouse. Table 1 shows that 13 out of 20 editing sites are located in the CDS and 12 of those editing events can cause an amino acid change.

Identification of genes with monoallelic expression in B. t. indicus $\times$ B. t. taurus conceptuses

As the RDDs were enriched with A-to-I editing sites, we used only genomic variants for the identification of monoallelically expressed genes. We first assigned parental origin of each allele within the $\mathrm{F}_{1}$ RNAseq reads by determining which SNPs 
Table 1. A-to-I RNA editing sites in bovine that are conserved in human and/or mouse

\begin{tabular}{|c|c|c|c|c|c|}
\hline Chromosome & Tissue type & Gene & Gene section & Outcome & Conservation \\
\hline Chr3:9479773 & $\begin{array}{c}\text { Brain, kidney, liver, } \\
\text { skeletal muscle, placenta }\end{array}$ & COPA & CDS & Nonsyn (Ile->Val) & Human \\
\hline Chr4:114454773 & Skeletal muscle, placenta & FASTK & Intron & Unknown & Human, mouse \\
\hline Chr5:76189492 & Brain & ELFN2 & Intron & Unknown & Human \\
\hline Chr7:71024261 & Brain & CYFIP2 & CDS & Nonsyn (Lys->Glu) & Human, mouse \\
\hline Chr9:48598875 & Brain & GRIK2 & Intron & Unknown & Human, mouse \\
\hline Chr9:48598884 & Brain & GRIK2 & Intron & Unknown & Human \\
\hline Chr9:48598902 & Brain & GRIK2 & Intron & Unknown & Human, mouse \\
\hline Chr9:48600509 & Brain & GRIK2 & Intron & Unknown & Human \\
\hline Chr9:48600848 & Brain & GRIK2 & CDS & Nonsyn (Gln->Arg) & Human, mouse \\
\hline Chr9:48600865 & Brain & GRIK2 & CDS & Syn (Gly->Gly) & Human \\
\hline Chr9:48634035 & Brain & GRIK2 & CDS & Nonsyn (Tyr->Cys) & Human, mouse \\
\hline Chr9:48634048 & Brain & GRIK2 & CDS & Nonsyn (Ile->Val) & Human, mouse \\
\hline Chr12:15671536 & $\begin{array}{c}\text { Brain, kidney, liver, } \\
\text { skeletal muscle, placenta }\end{array}$ & COG3 & CDS & Nonsyn (Ile->Val) & Human, mouse \\
\hline Chr13:67116409 & $\begin{array}{c}\text { Brain, skeletal muscle, } \\
\text { placenta }\end{array}$ & $B L C A P$ & CDS & Nonsyn (Lys->Arg) & Human \\
\hline Chr13:67116439 & $\begin{array}{l}\text { Brain, kidney, skeletal } \\
\text { muscle, placenta }\end{array}$ & $B L C A P$ & CDS & Nonsyn (Gln->Arg) & Human, mouse \\
\hline Chr13:67116448 & Brain, kidney & $B L C A P$ & CDS & Nonsyn (Tyr->Cys) & Human, mouse \\
\hline Chr17:42806463 & Brain & GRIA2 & Intron & Unknown & Human, mouse \\
\hline Chr17:42806724 & Brain & GRIA2 & CDS & Nonsyn (Gln->Arg) & Human, mouse \\
\hline Chr22:39005364 & Brain & CADPS & CDS & Nonsyn (Glu->Gly) & Human, mouse \\
\hline ChrX:34866822 & Brain & GABRA3 & CDS & Nonsyn (lle->Met) & Human, mouse \\
\hline
\end{tabular}

Nonsyn: nonsynonymous editing; Syn: synonymous editing 
were found in the sire's DNAseq reads. As illustrated in Figure S5, the only SNPs that were used to assign parental alleles were those that were homozygous in the $B$. $t$. indicus sire and that had at least one allele different from the allele found in the sire in the $\mathrm{F}_{1}$ RNAseq data. Following assignment of the parental alleles, allele-specific read counts covering each of the SNPs in the same gene were aggregated as previously described (DeVeale et al., 2012) and a binomial test was used to detect consistent allelic expression bias (false discovery rate $(\mathrm{FDR})<0.05$; Table S3 and S4). As the annotation of the bovine genome is imperfect, we further manually annotated the candidate genes using the Integrative Genomic Viewer (IGV; Robinson et al., 2011). We identified a total of 53 genes subject to monoallelic expression in at least one of the analyzed tissues (i.e., 33 in brain, 36 in kidney, 38 in liver, 36 in muscle, and 35 in placenta; Table S3 and S4; link to Table S3-4: http://www.tandfonline.com/doi/full/10.1080/15592294.2016.1184805). Of the identified 53 genes, 23 were known to be imprinted in the cow (also in human and/or mouse; http://igc.otago.ac.nz/; http://www.har.mrc.ac.uk/research/genomic_imprinting/) and 7 had previously been characterized as imprinted in human and/or mouse but had not been reported to be imprinted in cow (Table S5; link to Table S5: http://www.tandfonline.com/doi/full/10.1080/15592294.2016.1184805). Of the remaining 23 genes, 10 were uncharacterized or unannotated transcripts located in known imprinted clusters, whereas the other 13 genes were distributed throughout the bovine genome and were not located close to any known imprinted clusters (Table S5). 
Validation of the imprinted genes previously identified in human and/or mouse

We used PCR followed by Sanger sequencing to validate the allele specific expression of GNASXL (GNAS/GNASXL domain), COPG2IT1 (MEST/COPG2 domain), MEG8 and MEG9 (GTL2/DLK1 domain), MIMT1, USP29, ZIM2 (PEG3/USP29 domain), PON3 and PPP1R9A (PEG10/SGCE domain), and MKRN3 (SNRPN domain). The other 20 imprinted genes (Table S5) were not validated because we have previously described these genes to be imprinted (Chen et al., 2015). In the GNAS domain, we identified GNASXL as a paternally expressed transcript isoform which has a unique first exon but shares identical sequences from exon 2 to the end of the transcript with the maternally expressed transcript GNAS (also known as NESP55 in cattle [NM_001271771.1], Fig. 3A). By using the SNP located in the last exon of these transcripts (Fig. 3A) we validated the paternal expression of GNASXL and maternal expression of GNAS (Fig. 3B). In addition, analyses of two other SNPs (one specific to the GNASXL first exon and the other specific to the GNAS first exon) confirmed this result (Fig S6A). Further, DNA methylation of the GNASXL and GNAS promoter regions was confirmed using bisulfite mutagenesis followed by cloning and sequencing. The GNASXL differentially methylated region (DMR; the locus' putative ICR) showed hypomethylation of the paternal allele and hypermethylation of the maternal allele, which was associated with the paternal expression of this transcript (Fig. 3C and Fig. S6B). To the contrary, the GNAS DMR was methylated on the paternal allele while unmethylated on the maternal allele, which was associated with the maternal expression of GNAS (Fig. $3 \mathrm{C}$ and Fig. S6B). In addition to GNASXL and GNAS, we verified the maternal expression of MEG8, MEG9, PPPIR9A, PON3 and ZIM2, and paternal expression of 
Figure 3.

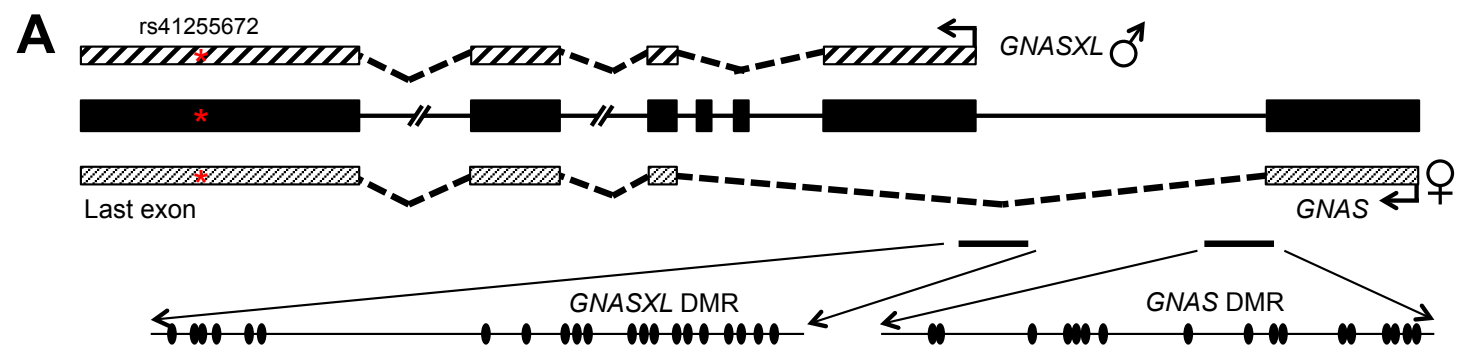

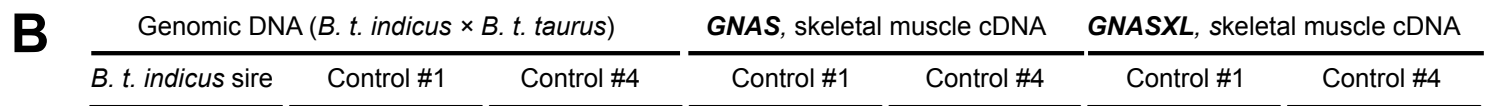

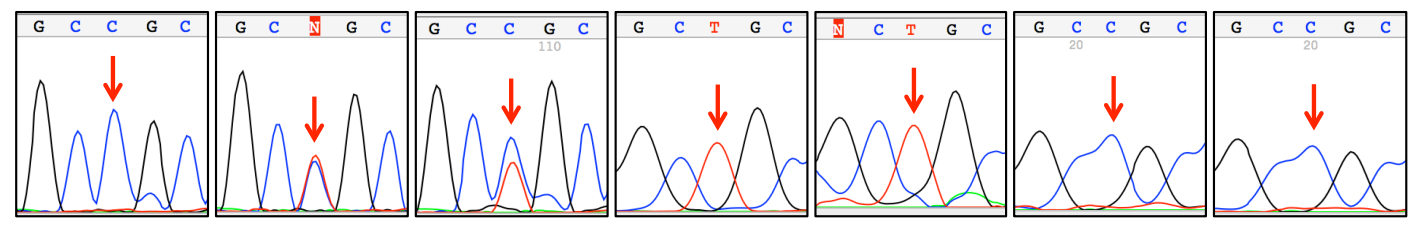

C
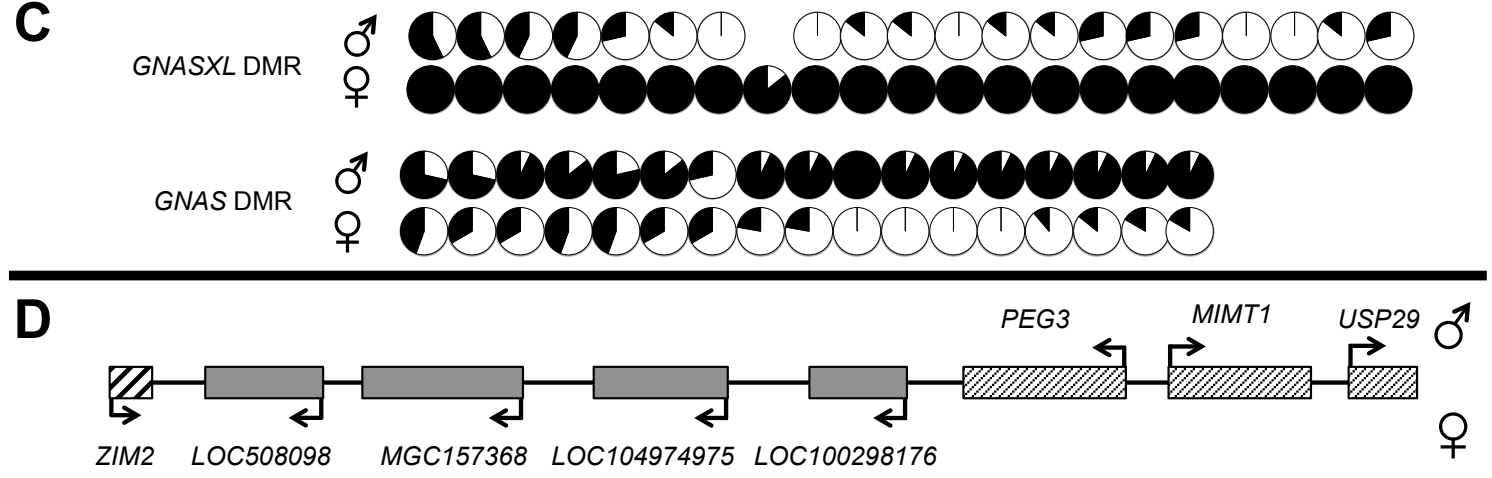

$E$

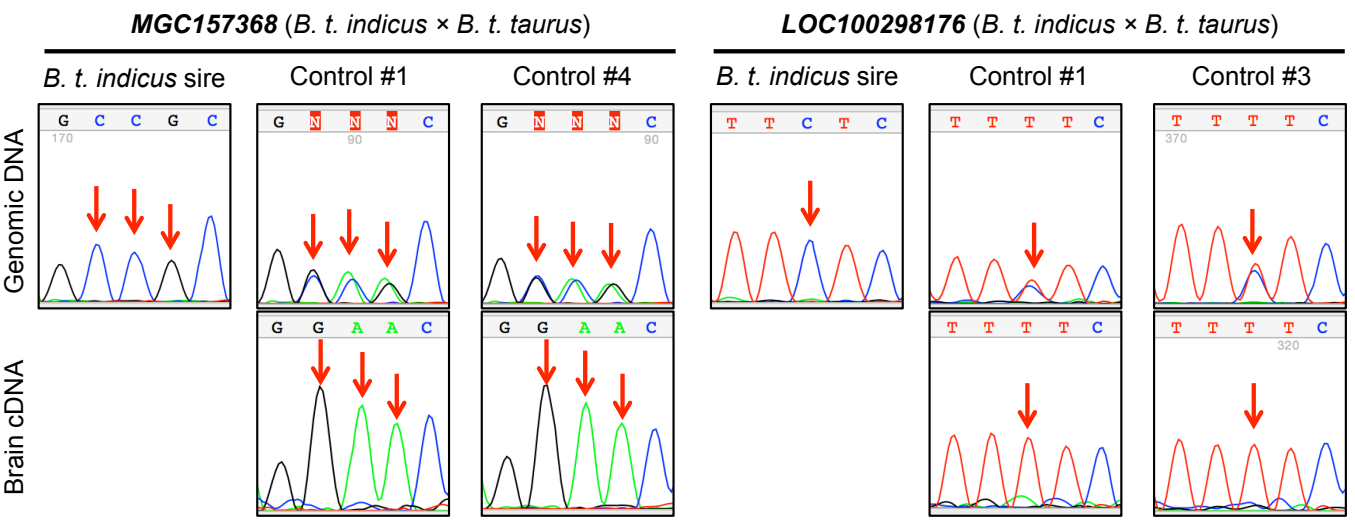


Figure 3. Validation of the allele-specific gene expression in $G N A S / G N A S X L$ and $P E G 3 / U S P 29$ imprinted clusters in day $\sim 105$ B. t. indicus $\times$ B. t. taurus $\mathbf{F}_{\mathbf{1}}$ conceptuses. A). Schematics of the GNAS/GNASXL loci (not all exons are shown and are not drawn to scale). Bent arrows represent the transcription start sites. Patterned boxes above and below the gene structure (in black) represent the GNASXL and GNAS transcripts, respectively. The red asterisk in the last exon represents the SNP used to discriminate the parental alleles for allele-specific expression analyses in panel $\mathbf{B}$. The black ovals represent the $\mathrm{CpGs}$ found within the amplicon used for DNA methylation analyses (i.e., panel C). B). Validation of the maternal expression of GNAS and paternal expression of GNASXL by Sanger sequencing. The SNP sites are indicated by red arrows. Left: genotyping results obtained by Sanger sequencing of the $B$. $t$. indicus sire and the $\mathrm{F}_{1}$ individuals at the SNP site. Middle and right: Sanger sequencing results of RT-PCR amplicons of GNAS and GNASXL. Validation of the allelic expression of GNAS and GNASXL at other SNP sites may be found in Figure S6A. C). DNA methylation analyses of GNASXL (top) and GNAS (bottom) differentially methylated regions (DMRs). DNA methylation was determined using bisulfite mutagenesis, cloning, and Sanger sequencing. Parentalorigin of each clone was determined using heterozygous SNPs in the $\mathrm{F}_{1}$ individuals. Methylation of $\mathrm{CpG}$ sites for each parental allele was summarized from at least 7 clones and is presented with pie charts with black representing the percent of methylated cytosines and white representing the percent of unmethylated cytosines at each site. The complete bisulfite maps may be found in Figure S6B. D). Schematics of the PEG3/USP29 imprinted cluster. Bent arrows represent the transcription direction of each gene. E). Validation of maternal expression of MGC157368 and LOC100298176. Top panels for each gene are the genotyping results obtained by Sanger sequencing of the $B$. $t$. indicus sire and $\mathrm{F}_{1}$ individuals. Bottom panels are the Sanger sequencing results of the RT-PCR amplicons of $M G C 157368$ and LOC100298176. The validation of the allelic expression of other genes in the PEG3/USP29 imprinted cluster may be found in Figure S8. $\mathbb{\mathscr { K }}$ = genes previously identified to be imprinted in human, mouse, and bovine. $\square Z Z$ = genes previously identified to be imprinted in human and/or mouse but not reported to be imprinted in bovine. uncharacterized transcripts in known imprinted clusters. 
COPG2IT1, MIMT1, MKRN3, and USP29 in B. t. indicus $\times$ B. t. taurus $\mathrm{F}_{1}$ hybrids (Fig. S7-S9).

Validation of the monoallelic expression of the uncharacterized/unannotated transcripts in previously known imprinted clusters

We identified 10 uncharacterized/unannotated transcripts with monoallelic expression in previously known imprinted clusters, of which four transcripts were located in the PEG3/USP29 cluster and six transcripts were in the SNRPN domain. In the PEG3/USP29 domain, the four uncharacterized transcripts (i.e., LOC508098, MGC157368, LOC104974975, and LOC100298176) were located downstream of the paternally expressed gene $P E G 3$ and upstream of the maternally expressed gene ZIM2 (Fig. 3D). We verified the maternal expression of LOC508098, MGC157368, LOC104974975, and LOC100298176 (Fig. 3E, Fig. S8, Table S3 and S5). According to RefSeq gene annotation (Pruitt et al., 2014), LOC508098, MGC157368, and LOC104974975 are protein-coding genes. In addition, LOC100298176 has high proteincoding potential ( 0.78 coding probability) based on the Coding Potential Assessment Tool (CPAT; Wang et al., 2013). We considered a transcript with CPAT coding probability $>0.348$ to possess protein-coding potential as previously reported for bovine (Billerey et al., 2014).

In the SNPRN domain, LOC100848941 and LOC101907203 were identified as two paternally expressed noncoding RNAs (ncRNAs) located downstream of the maternally expressed gene $U B E 3 A$ (Fig. S9). In addition, two paternally expressed ncRNAs (LOC100849023 and LOC101907679; Fig. 4A left panel) located upstream of 
$U B E 3 A$ were identified (Fig. S9). Finally, we verified paternal expression of an unannotated ncRNA (CPAT coding probability 0.06; Wang et al., 2013) with cuffmerge gene ID XLOC_045114 (Chr21:350,550-552,040 bp, UMD3.1) which was located $\sim 300 \mathrm{~kb}$ upstream of the paternally expressed gene $S N R P N$ (Fig. S9). To determine whether XLOC_045114 has any human/mouse counterpart, we aligned its cDNA sequence to the human and mouse genomes using BLAST but were unable to confidently identify any matches.

Validation of monoallelically expressed genes not found in known imprinted clusters

We identified 13 genes with monoallelic expression that were located $>15 \mathrm{Mb}$ distance from any known imprinted clusters. For these genes, nine (i.e., AOX1, APCS, $A S 3 M T, C 1 R, C 1 S, C D A, K R T 7, O O E P$, and $R D H 16$ ) were annotated protein-coding genes, of which $A P C S, A S 3 M T$, ClR, CIS, CDA, OOEP, and RDH16 were monoallelically expressed from the B.t.taurus allele (maternal), while AOXI and KRT7 were expressed from the B. t. indicus allele (paternal) (Fig. 4C and Fig. S10 left panels and Table S3). The remaining four genes were either uncharacterized ncRNA (i.e., LOC101905472) or unannotated transcripts: XLOC_009410 (Chr4:59,913,25359,913,967, UMD3.1, CPAT coding probability: 0.38), XLOC_012439 (Chr5:115,431,044-115,432,886, UMD3.1, CPAT coding probability: 0.36), and XLOC_052524 (ChrX:113,053,268-113,055,164, UMD3.1, CPAT coding probability: 0.02). Consistent with RNAseq analysis, Sanger sequencing confirmed the maternal expression of XLOC_052524, and paternal expression of LOC101905472, 


\section{Figure 4.}

A

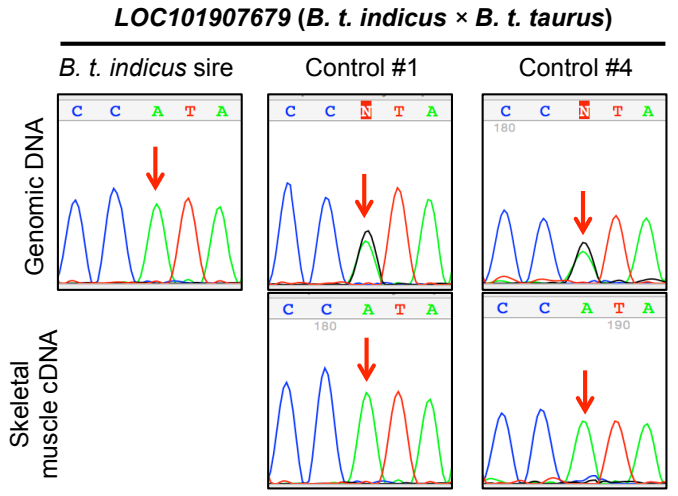

B

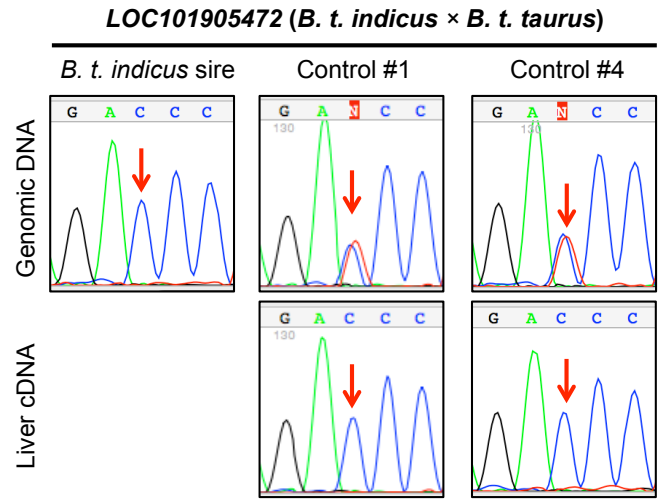

C

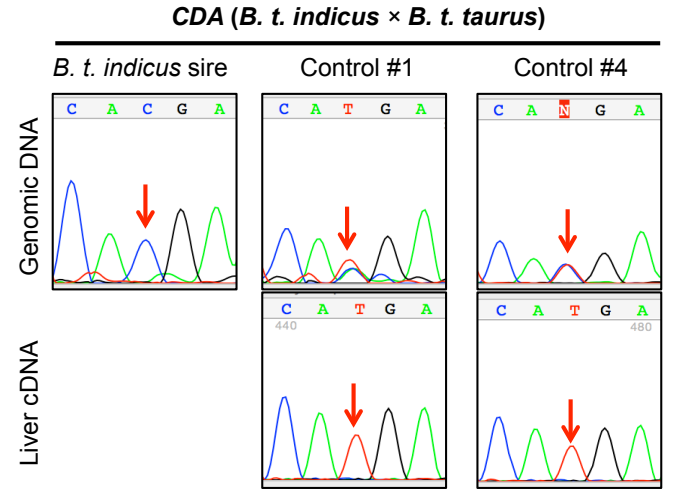

LOC101907679 (B. t. taurus $\times$ B. t. taurus)

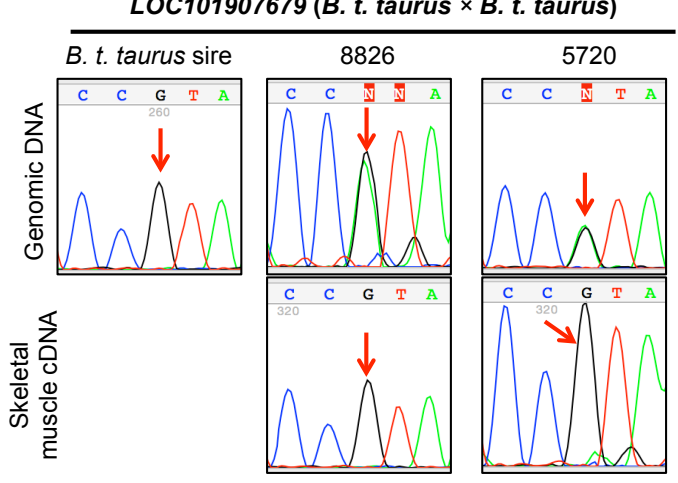

LOC101905472 (B. t. taurus $\times$ B. t. taurus)

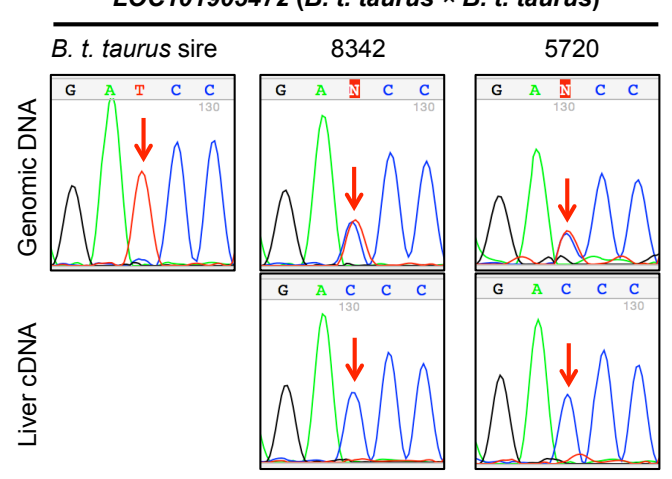

CDA (B.t. taurus $\times$ B. t. taurus)

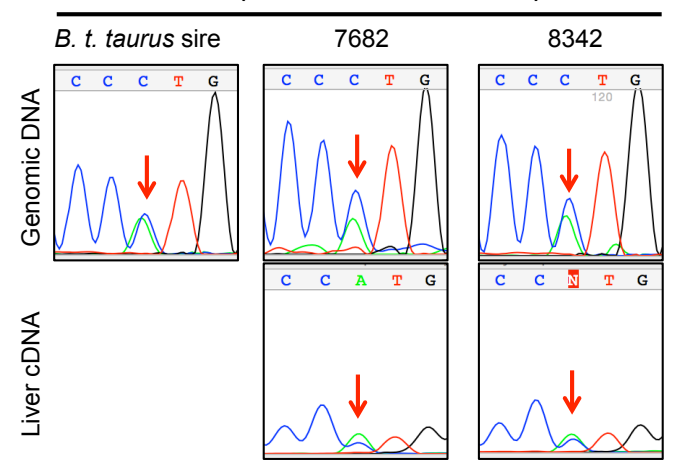


Figure 4. Corroboration of the allele specificity in gene expression identified by RNAseq. A-C). left panels show results obtained from the day $\sim 105$ B.t. indicus $\times$ B.t.taurus $\mathrm{F}_{1}$ conceptuses. A-C). right panels show results obtained from the day 86 B.t.taurus $\times$ B. t. taurus individuals. For each gene, the top lines are the genotyping results of the $F_{1}$ s and their sire while the bottom lines are the Sanger sequencing results of the RT-PCR amplicons of the $\mathrm{F}_{1}$ conceptuses. A). An example of a gene that had parental-origin-dependent monoallelic expression. LOC101907679, an uncharacterized transcript in the SNRPN imprinted cluster was paternally expressed in both B.t. indicus $\times$ B. t. taurus and B.t.taurus $\times$ B. t. taurus individuals. B). An example of a gene that had sequence-dependent monoallelic expression. For LOC101905472, the "C" allele was always predominantly expressed, regardless of its parentalorigin. C). An example of a gene (i.e., $C D A$ ) that was monoallelically expressed in B.t. indicus $\times$ B. t. taurus $\mathrm{F}_{1}$ hybrids but biallelically expressed in B.t.taurus $\times$ B. t. taurus individuals. Further validations of allele-specific expression in B.t. indicus $\times$ B. t. taurus $\mathrm{F}_{1} \mathrm{~s}$ and B. t. taurus conceptuses may be found in Figure S10-11. 
XLOC_012439, and XLOC_009410 in the B.t. indicus $\times$ B.t.taurus $\mathrm{F}_{1}$ hybrids (Fig. 4B and Fig S10 left panels and Table S3).

Corroboration of the allele-specificity of gene expression in day 86 B. t. taurus $\times$

\section{B. t. taurus conceptuses}

Beyond genomic imprinting, cis-eQTL (cis-regulating expression quantitative trait loci) can also induce biased allelic gene expression (Wang and Clark, 2014). As we lack the ability to produce the reciprocal B. t.taurus $\times$ B.t. indicus $\mathrm{F}_{1}$ conceptuses, we used tissues from nine day 86 B.t.taurus $\times$ B. t. taurus female conceptuses (Holstein breed all sired by bull: 507HO10723 DE-SU 527 SPUR-ET) to corroborate allelic specificity of transcription of genes not previously reported to be imprinted in bovine ( $\mathrm{n}=33$; Table S5). We used PCR followed by Sanger sequencing to identify informative SNPs in the B.t.taurus $\times$ B.t.taurus conceptuses. Of the analyzed 33 genes we found 20 genes with heterozygous SNPs in at least one conceptus (Fig 4, Fig S10 and S11 right panels). Of these, nine genes (i.e., COPG2IT1, GNAS, GNASXL, MEG8, LOC101907679, LOC100298176, LOC104974975, LOC1009849023, MEG8, and XLOC_052524) showed consistent parental-allele-specific gene expression between the B.t. indicus $\times$ B.t.taurus and B.t.taurus $\times$ B.t.taurus conceptuses while $R D H 16$ and CDA were found to be biallelically expressed and C1R, C1S, LOC101905472, and XLOC_012439 were monoallelically expressed but not imprinted (Fig 4, Fig S10 and S11). We were not able to assign parental origin to the monoallelically expressed APCS, ZIM2, USP29, LOC508908, and XLOC_009410 alleles as the sire was heterozygous at these loci (Fig. 5, Fig. S10 and S11). Using this scheme, eight novel imprinted genes were identified (Fig. 5B) and imprinted expression of another 23 genes 

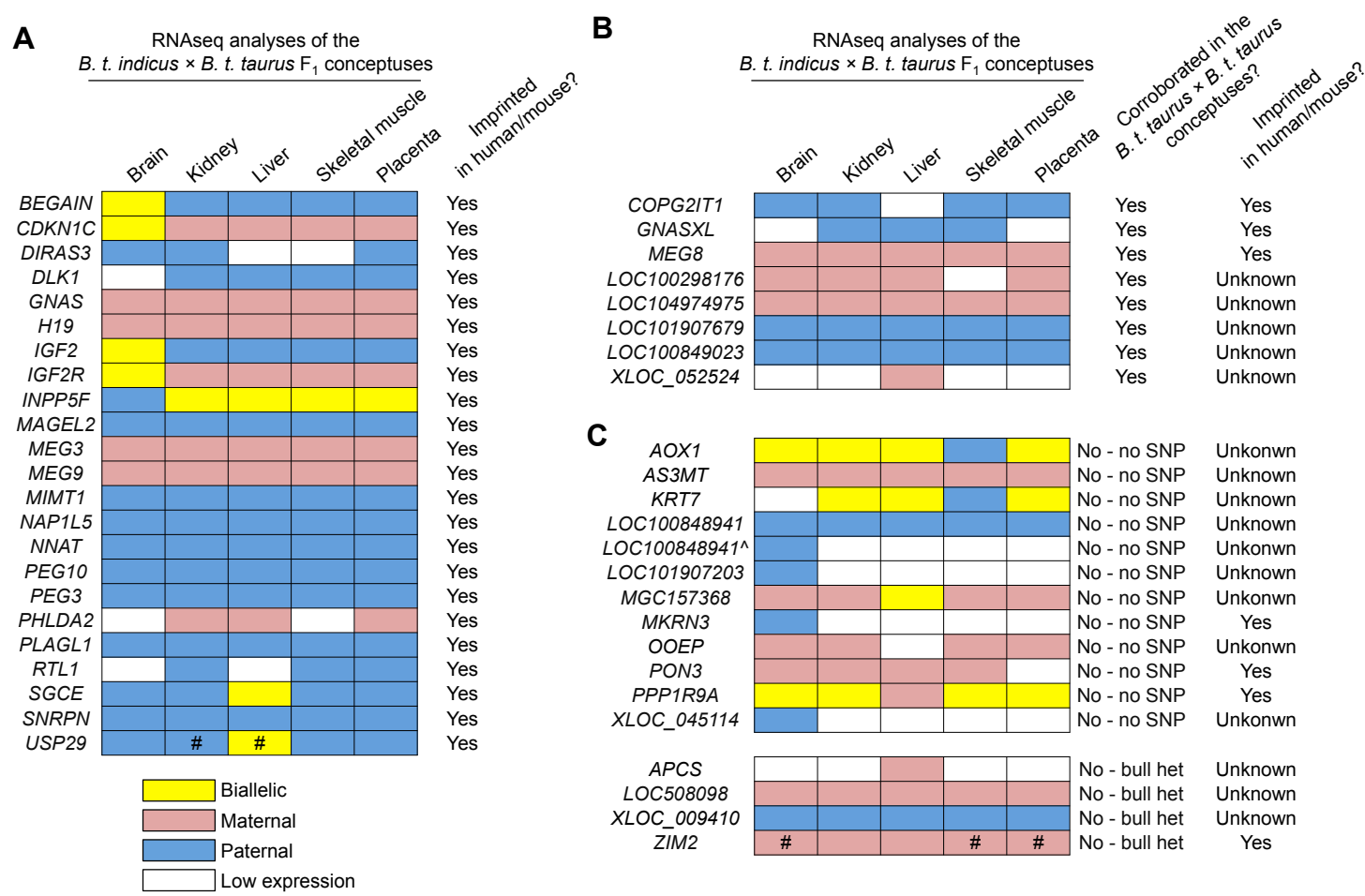

Figure 5. Summary of the imprinted and monoallelically expressed genes identified in the current study. For A-C), different colors denote the type of expression of listed genes for each analyzed tissue. A). Genes previously reported to be imprinted in bovine. B). Genes not previously reported to be imprinted in bovine. C). Genes with monoallelic expression in the B. t. indicus $\times$ B.t.taurus $\mathrm{F}_{1} \mathrm{~s}$ but which parental origin of the transcript could not be corroborated due to lack of informative SNPs in the B. t. taurus $\times$ B. t. taurus conceptuses or as a result of the heterozygosity of their sire. $\#=$ genes with inter-individual variations in allele-specific gene expression. LOC100848941^ ${ }^{\wedge}$ is an isoform of LOC100848941 (SNPRN imprinted cluster) only identified in brain. Allele-specific read counts and allelic expression ratios of each gene may be found in Tables S3. 
in cattle was corroborated (Fig. 5A). RNAseq results indicated that 16 of these genes showed allele-specific expression in all analyzed tissues, seven had biallelic expression in at least one tissue, and eight were lowly expressed in some tissues (Fig. 5A and 5B). We also identified a paternal transcript in the $S N R P N$ domain (referred here as "LOC100848941 brain isoform") which connected the two paternal transcripts LOC100848941 and LOC101907203 (Fig. S9 top panel).

\section{Allele-specific expression analyses of X-linked genes in bovine placenta}

To determine whether X-chromosome inactivation (XCI) in bovine placenta is random or imprinted (Dindot et al., 2004; Xue et al., 2002), allele expression ratios for the annotated X-linked genes were analyzed in placenta of the four B.t. indicus $\times$ B.t. taurus $\mathrm{F}_{1}$ female conceptuses. If the $\mathrm{XCI}$ is imprinted, we expect that most of the $\mathrm{X}$ linked genes would be expressed from the maternal allele, except for those genes that escape XCI (Wang et al., 2012). However, if XCI is random, we expect that both parental alleles of the X-linked genes would be expressed and that allele expression ratios would be variable among individuals due to the sampling effects (Wang et al., 2012). We plotted the distribution of the allele expression ratios for both autosomal genes and Xlinked genes in placenta of the four B.t. indicus $\times$ B. t. taurus $\mathrm{F}_{1}$ conceptuses (Fig. 6A). For autosomal genes, the distribution of the allele expression ratios of all four individuals had an approximate 50:50 ratio. However, the X-linked genes showed biallelic expression but with inter-individual variation of the allele expression ratios (mean ranges from 29.0 to $66.7 \%$; Fig. 6 A), which is consistent with the distribution of the allele expression ratios for the $\mathrm{X}$-linked genes in the mule and horse placenta (Wang et al., 2012). We further analyzed the allele expression ratios of $14 \mathrm{X}$-linked genes that have 


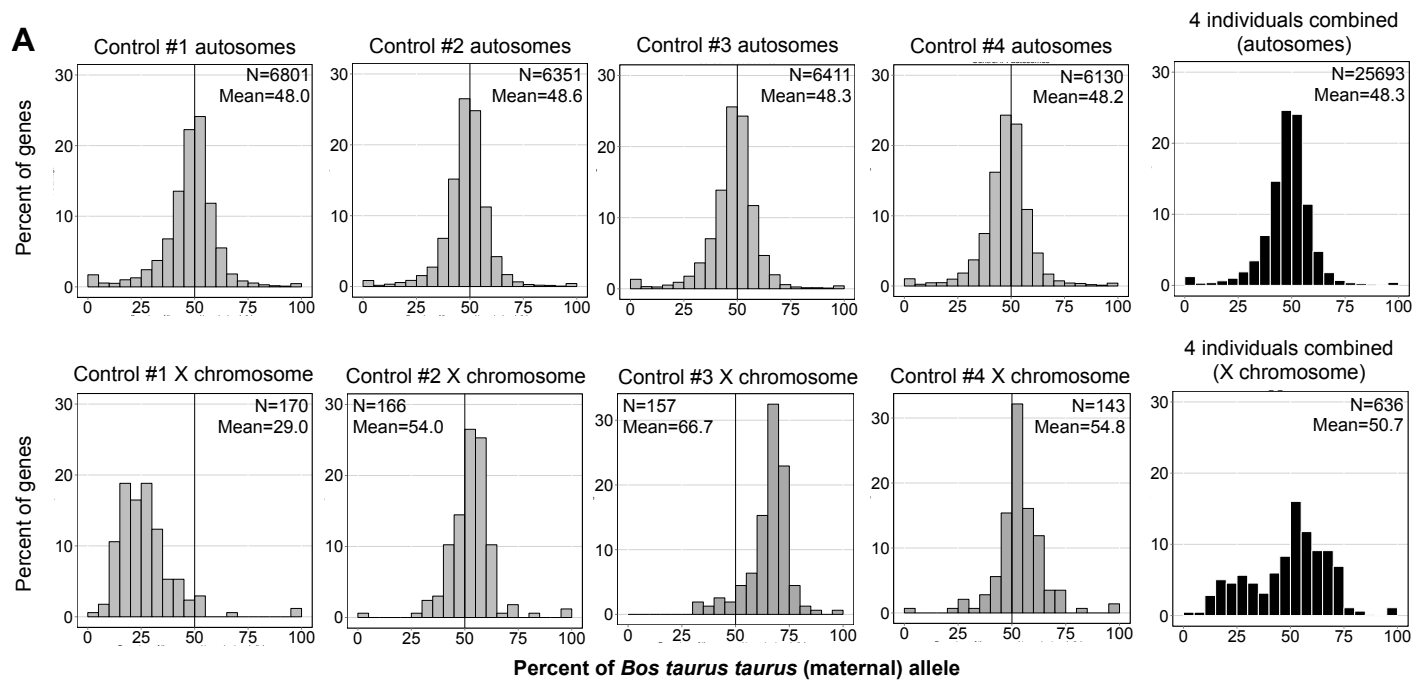

B

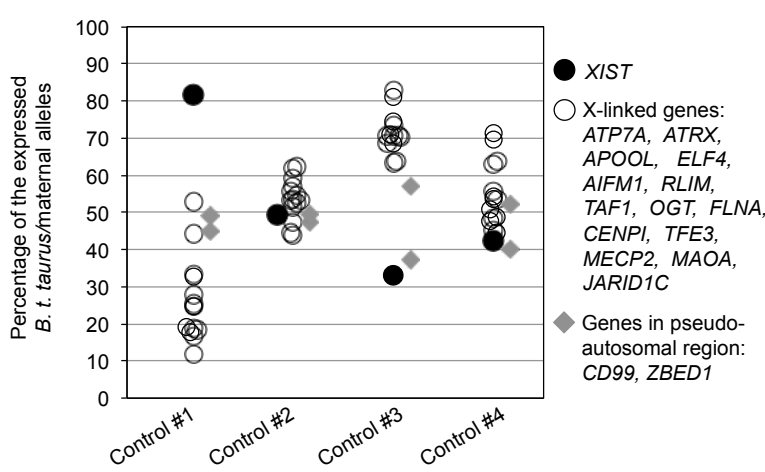

C

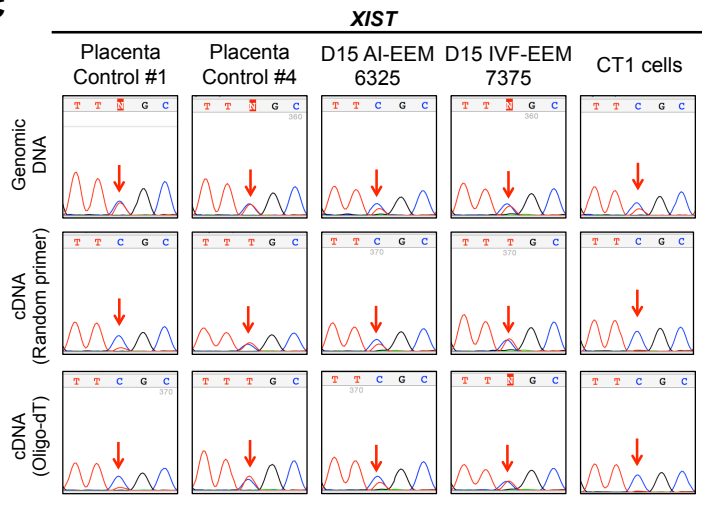

Figure 6. Allele-specific expression analyses for autosomal and $X$-linked genes in placental membranes from day $\sim 105$ B. t. indicus $\times$ B. t. taurus $F_{1}$ hybrids. The placental membranes used for this study were the intercotyledonary region of the chorioallantois. A). Histograms of the allelic expression ratios for the autosomal genes (top) and X-linked genes (bottom) in placenta from each conceptus. The $\mathrm{Y}$-axis represents the percent of genes with varied levels of expression from the $B$. $t$. taurus (maternal) allele (X axis). $\mathrm{N}=$ number of annotated genes with informative SNPs. Mean $=$ the mean of the percent allelic expression. B). Plot of allelic expression ratios of XIST (filled circles), two pseudo-autosomal genes (gray diamonds), and $14 \mathrm{X}$-linked genes (open circles) from the placenta RNAseq data. The X-linked genes have previously been described as being subjected to imprinted X chromosome inactivation in mouse. The detailed allele-specific read counts of these genes may be found in Table S6. C). Sanger sequencing results of XIST allelic expression in the B. t. indicus $\times$ B. $t$. taurus $\mathrm{F}_{1}$ placenta, in in vivo- (AI) and in vitro- (IVF)-produced day 15 (D15) bovine extraembryonic membranes (EEM), and CT1 trophectoderm cell line. Top row is the genotyping results while the two bottom rows are sequencing results for the RT-PCR amplicons. Both random primers and oligo(dT) were used for cDNA synthesis. 
previously been described as imprinted in mouse placenta (McGraw et al., 2013) and/or extra-embryonic cell lines (Merzouk et al., 2014), two genes (CD99 and ZBED1) found in the pseudoautosomal region (PAR; Das et al., 2009), and the ncRNA XIST which is expressed from the inactive X (Lee and Bartolomei, 2013). We observed that similar to the X-linked genes, XIST was expressed from both parental alleles (Fig 6B and C, Table S6). Further, we found an anti-association between the allelic expression ratio of XIST and the X-linked genes in two of the samples (Fig 6B and Table S6). Conversely to XIST and the $\mathrm{X}$-linked genes, the allele expression ratios of the PAR genes $C D 99$ and ZBED1 centered toward 50:50 with no apparent association with X-linked genes or XIST expression (Fig. 6B). For this study the placental samples were taken from the intercotyledonary chorioallantois for which predecessor cells come from the trophectoderm and the epiblast (Bianchi et al., 1993; Luckett, 1978), therefore, the possibility exists that the randomness of $\mathrm{X}$-linked gene allele expression observed here was simply the result of the expression observed in tissues derived from the inner cell mass (Lee and Bartolomei, 2013). To refine our findings, we turned to day 15 bovine extraembryonic membranes (EEM)(Dobbs et al., 2014) and CT1 cells (Talbot et al., 2000), a trophectoderm cell line derived from a single bovine female blastocyst. The EEM were dissected from the female conceptuses by removing the embryonic disk and should contain no epiblast derivatives(van Leeuwen et al., 2015). Sanger sequencing results showed that XIST was expressed from both parental alleles in EEM of the four day 15 conceptuses analyzed (Fig. 6C and Fig. S12), indicating random XCI in bovine extraembryonic membranes. However, in CT1 cells only one allele of XIST was 
expressed. It is possible that this "monoallelic" XIST expression resulted from the clonal expansion of the cells that randomly inactivate one of the $\mathrm{X}$ chromosomes.

\subsection{Discussion}

To date, approximately 150 imprinted genes have been identified in humans and mice (http://igc.otago.ac.nz; http://www.har.mrc.ac.uk/research/genomic_imprinting/), but less than 30 genes have been described as imprinted in cattle (Tian, 2014). In this

study, we advance the genomic imprinting field by the de novo identification of imprinted genes in bovine using RNAseq. To do so, we identified parent-specific SNP alleles in RNAseq data after filtering A-to-I RNA editing sites and determined global allelespecific gene expression in B.t. indicus $\times$ B. t. taurus $\mathrm{F}_{1}$ conceptuses. We also corroborated the allele specificity of the identified monoallelically expressed genes using B. t. taurus $\times$ B. t. taurus progenies to exclude potential cis-eQTL effects on allelic expression. These analyses confirmed the allelic origin of 23 transcripts previously reported to be imprinted in bovine and identified eight novel imprinted genes in the bovine conceptuses. Of note, we were not able to corroborate the allele-specific expression of 16 genes in the B.t.taurus $\times$ B.t.taurus conceptuses because of a lack of informative SNPs. However, of these 16 genes, PPPIR9A, PON3, MKRN3, and ZIM2 have previously been reported to be imprinted in human and/or mouse and showed consistent allele-specific expression in the $B$. t. indicus $\times$ B. t. taurus $\mathrm{F}_{1} \mathrm{~s}$, which together suggest that these four genes are also imprinted in bovine. 
Most imprinted genes are organized in megabase-sized clusters. Within the cluster is a differentially methylated imprinting control region that regulates the proper allelic expression of the imprinted genes across the entire domain (Edwards and Ferguson-Smith, 2007; Wan and Bartolomei, 2008). Of the 53 monoallelically expressed genes identified in this study, 30 genes have previously been reported to be imprinted in humans and/or mice and 24 are distributed in eight imprinted clusters namely; GNAS/GNASXL domain, PEG3/USP29 domain, GTL2/DLK1 domain, PEG10/SGCE domain, MEST/COPG2 domain, SNRPN domain, H19/IGF2 domain, and KCNQ1 domain. Further, ten previously uncharacterized/unannotated transcripts were identified to be subject to allele-specific expression in two known imprinted clusters (i.e., four transcripts in PEG3/USP29 domain and six in the $S N R P N$ domain) in the $B . t$. indicus $\times$ B.t. taurus conceptuses. Of these 10 transcripts, LOC100298176 and LOC1014974975 (within the PEG3/USP29 domain) and LOC101907679 and LOC100849023 (within the SNRPN domain) were demonstrated to be imprinted in the bovine conceptuses. For the remaining six transcripts, parental-specificity could not be ascertained due to a lack of informative SNPs in the B. t. taurus $\times$ B. t. taurus conceptuses. However, we suggest that these genes are also imprinted in bovine because of the proximity of these uncharacterized/unannotated transcripts to known imprinted genes is less than $100 \mathrm{~kb}$ and they are within known and well characterized clusters. In addition, in the $S N R P N$ domain, the last exon of the antisense $L O C 100848941$ brain isoform extends to the $U B E 3 A$ gene. It is tempting to speculate that the paternal expression of this transcript may be involved in the repression of the paternal copy of 
$U B E 3 A$ as is observed for $U B E 3 A-A T S$ in human (Rougeulle et al., 1998) and mouse (Meng et al., 2012).

Gene expression can be affected by genetic variants. cis-eQTL refers to the class of genetic variants that affect gene expression on the same DNA molecule thus leading to allele-specific or allele-biased gene expression (Rockman and Kruglyak, 2006). To determine whether monoallelic gene expression identified in the B.t. indicus $\times$ B. $t$. taurus $\mathrm{F}_{1} \mathrm{~s}$ was due to cis-eQTL effects or genomic imprinting, we corroborated the parent-specificity of the transcripts in B.t.taurus $\times$ B.t. taurus progenies as we lack the ability to produce reciprocal B. t. taurus $\times$ B. $t$. indicus crosses. Our findings confirmed that monoallelic gene expression is not necessarily the result of genomic imprinting (Wang and Clark, 2014). For the example of LOC101905472, it is always the "C" allele that is predominantly expressed regardless of its parental-origin in both $B$. t. indicus $\times B$. t. taurus and B. t. taurus $\times$ B. t. taurus conceptuses. Further, $C D A$ and $R D H 16$ showed monoallelic expression in $B$. $t$. indicus $\times B$. t. taurus conceptuses but biallelic expression in B. t. taurus $\times$ B. t. taurus progenies. Our results demonstrate that strong cis-eQTL could lead to monoallelic gene expression and highlight the importance of the determination of allele-specific gene expression using different mating structures crosses prior to claiming that any monoallelically expressed genes are imprinted.

Genome-wide assessment of imprinted gene expression in non-inbred organisms such as cattle and humans can be challenging, as heterozygous SNPs are not always informative of parental-origin of an allele. We were unable to assign the allele-origin of a heterozygous SNP in the B.t. indicus $\times B$. t. taurus $\mathrm{F}_{1} \mathrm{~s}$ if the $B . t$. indicus sire was heterozygous at the same site. Discovery of imprinted genes using RNAseq relies on 
aligning reads to a reference genome, however, some bovine sequences have recently been shown to be absent or misassembled in the current bovine reference genome UMD3.1 (Whitacre et al., 2015) and perfection of the reference assembly will help improve the RNAseq-based identification of imprinted genes in cattle. Further, technical limitations of standard RNAseq may also preclude the comprehensive discovery of imprinted genes. For example, the identification of antisense imprinted transcripts such as $A P E G 3$, a paternally expressed antisense transcript in the PEG3 locus (Choo et al., 2008), is not possible as our samples were not sequenced using a strand-specific RNAseq protocol (Levin et al., 2010). In addition, the procedures performed for RNA isolation for this study prevent us from identifying small imprinted RNAs as well as nonpolyadenylated molecules (e.g., some long ncRNAs). In future studies, the single-cell RNAseq technology will allow allele-specific gene expression analyses at exquisite resolution (Deng et al., 2014) and help identify genes that are only imprinted in specific cell types such as Ube3a in neurons (Yamasaki et al., 2003).

Advances in next generation sequencing technology have allowed the discovery of more than a million A-to-I RNA editing sites in human (Ramaswami et al., 2012; Ramaswami et al., 2013) and thousands of sites in mouse (Danecek et al., 2012; Neeman et al., 2006). Conversely to human and mouse, only 23 A-to-I RNA editing sites have been reported in cattle and these editing sites were identified as a result of searching evolutionarily conserved human/mouse A-to-I RNA editing sites in bovine brain RNAseq data (Pinto et al., 2014). In this study, we greatly expand the list by identifying 671potential A-to-I RNA editing sites in the bovine conceptuses using RNAseq. Consistent with the observation that most A-to-I editing sites in human and mouse are not 
located in coding regions ( $\mathrm{Li}$ and Church, 2013; Ramaswami et al., 2013), $\sim 96 \%$ of the identified editing sites in this study were in UTRs and introns. These editing events could either regulate RNA splicing processes (Rueter et al., 1999) or prevent the formation of double stranded RNA that may be recognized as non-self by the immune system (Liddicoat et al., 2015). We also found that $3 \%$ (20/671) of the editing sites identified in bovine are conserved in human and/or mouse and 11 of these sites are within neurotransmitter receptor genes such as GRIK2, GRIA2, and GABRA3. One of the notable conserved A-to-I RNA editing sites is in GRIA2 CDS region (Chr17:42,806,724; UMD3.1), which has $\sim 99 \%$ edited transcripts in brain and can result in the replacement of a glutamine residue by arginine in the GRIA2 protein. This editing site has been demonstrated to be the physiologically most important editing substrate of Adar2 because the seizure and lethality phenotype of Adar2 null mice can be reverted when the unedited Gria2 transcripts are replaced with the edited allele (Higuchi et al., 2000).

Imprinted X-chromosome inactivation in extraembryonic membranes has been described in mouse (Huynh and Lee, 2001) and rat (Wake et al., 1976). In bovine, a report showed that the $\mathrm{X}$-linked gene $M A O A$ is preferentially expressed from the maternal X-chromosome in term placenta suggesting imprinted X-inactivation in this species (Xue et al., 2002). Here we show that XIST is expressed from both parental chromosomes in epiblast-free (van Leeuwen et al., 2015)extraembryonic membranes from day 15 bovine conceptuses(Dobbs et al., 2014). From our results, we favor the hypothesis that $\mathrm{XCI}$ is random in bovine extraembryonic membranes and not imprinted as previously suggested. 
In summary, with this work we advance the field of genomic imprinting by expanding the list of imprinted genes in bovine and demonstrate that monoallelic gene expression can be due to cis-eQTL effects. In addition, our results greatly increase the number of identified A-to-I RNA editing sites in bovine conceptuses. We also show that $\mathrm{XCI}$ is random in bovine extraembryonic membranes. These findings will facilitate future studies to further dissect the molecular mechanisms and physiological roles of genomic imprinting and A-to-I RNA editing during fetal development of this economically important species.

\subsection{Materials and methods}

Whole genome sequencing of the B. t. indicus sire

Genomic DNA isolated from the semen of the B. t. indicus sire (Nelore breed; ABS CSS MR N OB 425/1 677344 29NE0001 97155) used to generate the B. t. indicus $\times$

B. t. taurus $\mathrm{F}_{1}$ hybrids was subject to DNAseq. In brief, two DNAseq libraries (one with 350 bp target insert size, the other with 550 bp target insert size) were prepared using Illumina TruSeq DNA PCR-Free Sample Preparation Kit according to the manufacturer's instructions (Illumina). Each DNAseq library was sequenced in a single lane with $2 \times 100$ bp paired-end reads on an Illumina HiSeq 2000 platform. One percent PhiX DNA was spiked into the bovine genomic DNA sample prior to library preparation to act as an internal control and allow the HiSeq optical system optimal base calling. A total of 347.3 million raw read pairs were obtained for genotyping the $B$. t. indicus sire after removing the DNAseq reads derived from PhiX DNA (Fig. S1). The raw FASTQ files 
are publically available at Gene Expression Omnibus (GEO accession no. GSE77389).

\section{Identification of the SNPs of B. t. indicus sire using DNAseq}

Raw DNAseq read pairs were subject to quality trimming prior to alignment to the reference genome (Fig. S1). The FastqMcf program (version: 1.04.636; https://code.google.com/p/ea-utils/wiki/FastqMcf) was used to remove the adaptor sequences in the reads. Further, DynamicTrim (version: 3.3.1; Cox et al., 2010) was used to obtain the longest segment of the read in which each base has $<1 \%$ error rate. After quality trimming, DNAseq read pairs were aligned to the bovine reference genome assembly UMD3.1 using Bowtie2 (version: 2.2.3; Langmead et al., 2009). Only read pairs that had the expected read mate orientation and expected ranges of insert size were retained for further analyses.

Following Bowtie2 alignment, Genome Analysis Tool Kit (GATK; version: 3.30; DePristo et al., 2011; McKenna et al., 2010) was used to further process the aligned read pairs for SNP calling. Known insertions/deletions (indel) and known SNPs downloaded from Ensembl and RefSeq FTP sites were supplied for insertions/deletions re-alignment and base quality recalibration (ftp://ftp.ensembl.org/pub/release78/variation/vcf/bos_taurus/; ftp://ftp.ncbi.nlm.nih.gov/snp/organisms/cow_9913/VCF/; $\mathrm{ftp}: / / \mathrm{ftp} . n c b i . n l m . n i h . g o v /$ snp/organisms/cow_30522/). After processing the aligned read pairs, GATK HaplotypeCaller was used to identify SNPs using the default parameters. Aligned read pairs subjected to indel realignment were also used for SNP calling by GATK HaplotypeCaller and SAMtools mpileup (version: 0.1.19; Li, 2011; Li et al., 
2009) without the base quality recalibration step in order to improve the identification of true SNPs.

Generation of the pseudo B. t. indicus genome and construction of a B. t. indicus/B. t. taurus diploid genome

A pseudo B. t. indicus genome was generated by editing the reference allele (UMD3.1; i.e., B. t. taurus) with the SNPs found in our B. t. indicus sire identified by the three SNP calling pipelines mentioned above (Fig. S1B, C, and D). The diploid genome was generated by combining the B. t. taurus and the pseudo B. t. indicus FASTA files.

RNAseq of the day $\sim 105$ B.t. indicus $\times$ B.t. taurus $F_{1}$ conceptuses

The four day $\sim 105$ B. t. indicus $\times$ B. t. taurus $\mathrm{F}_{1}$ conceptuses used in this study (i.e., Control \#1,\#2,\#3, and \#4) were previously described (Chen et al., 2013). These conceptuses shared the same B. t. indicus sire (ABS CSS MR N OB 425/1 677344 29NE0001 97155). RNAseq data of brain, kidney, liver, and skeletal muscle of the $F_{1}$ individuals were previously described (GEO accession number: GSE63509; Chen et al., 2015). In addition, for the current study, we generated RNAseq data from the placenta (intercotyledon regions) of these conceptuses following the RNA isolation, library preparation and sequencing procedures described previously (Chen et al., 2015). The raw FASTQ files are publically available at GEO database (accession no. GSE77389).

Alignment of RNAseq reads to the custom diploid genome

Prior to alignment, single-end RNAseq reads were processed as previously described (Chen et al., 2015). In brief, FastqMcf (version: 1.04.636; 
https://code.google.com/p/ea-utils/wiki/FastqMcf) and DynamicTrim (version: 3.3.1; Cox et al., 2010) programs were used to remove adaptor sequences and low quality bases in the reads, respectively. Only reads with a length greater than $30 \mathrm{bp}$ were used for alignment. The RNAseq reads were aligned to the custom diploid genome to minimize single reference genome alignment bias (Degner et al., 2009; Munger et al., 2014; Stevenson et al., 2013; Yuan et al., 2015).

HISAT2 (version: 2.0.0; Kim et al., 2015) was used to align RNAseq reads to the diploid genome using the following parameters: --score-min $L, 0,-0.2-m p 6,6-k n o w n-$ splicesite-infile known-splicesite-RefSeq-Ensembl.txt. These parameters require at least 96\% sequence identity for the RNAseq reads to be aligned. The known splicing junctions in Ensembl and RefSeq databases were downloaded from the Bovine Genome Database (http://www.bovinegenome.org; Elsik et al., 2015). The alignment of the RNAseq reads to the diploid genome was then merged to identify: i) reads that were aligned to the same single genomic position in both the B.t.taurus reference genome and the pseudo B. t. indicus genome, and ii) reads that were uniquely aligned to either genome but not aligned to the other.

\section{Transcript assembly and determination of transcript abundance}

Cufflinks (version: 2.2.1; Trapnell et al., 2010) was used for transcript assembly of each RNAseq library. Cuffmerge (version: 2.2.1; Trapnell et al., 2010) was used to generate a unified gene annotation for all libraries. Gene models generated by Cuffmerge were then compared to Ensembl and RefSeq gene models using Cuffcompare (version: 2.2.1; Trapnell et al., 2010). If a Cuffmerge gene model matched one Ensembl/RefSeq 
gene model (i.e., shared the same intron chain; class_code “=”), this Cuffmerge gene model was considered annotated. If a cuffmerge gene model did not match any Ensembl/RefSeq gene model (e.g., class_code “ $u$ ” or “ $x$ ”), it was considered unannotated. In addition, if a single Cuffmerge gene model matched more than one Ensembl/RefSeq gene model, the Cuffmerge gene model was replaced by the corresponding Ensembl/RefSeq gene models.

RNAseq read counts for each gene model were generated using HTSeq-count (version: 0.5.4; Anders et al., 2013) with mode: intersection-nonempty. Read counts were then normalized to the library size using Bioconductor package edgeR (version: 3.8.6; McCarthy et al., 2012; Robinson et al., 2010). For each tissue type, genes with $\geq 4$ counts/million (CPM) between all conceptuses were considered to be expressed and were retained for allele-specific expression analyses.

Identification of SNPS in the B.t. indicus $\times$ B.t.taurus $F_{1}$ conceptuses transcriptomes using RNAseq

The SNPiR pipeline (Piskol et al., 2013) was adapted to identify SNPs from the RNAseq data. The adapted SNPiR pipeline included 3 major steps: i) align RNAseq reads to the diploid UMD3.1/B. t. indicus genome using BWA mem (Li and Durbin, 2009), ii) call variants using GATK UnifiedGenotyper (McKenna et al., 2010), and iii) filter potential false positive variants (as described below). For read mapping, BWA mem (version: 0.7.7; Li and Durbin, 2009) was used to align the reads to the diploid genome using default parameters. As BWA does not align reads that span splice junctions, we next aligned the unmapped reads to the known splice junctions 
(http://www.bovinegenome.org; Elsik et al., 2015). The RNAseq reads that were successfully aligned to the diploid genome were then merged with the splice junction alignments. The uniquely aligned RNAseq reads were subjected to PCR duplicates removal_(only for the purpose of SNP identification), insertions/deletions re-alignment, and base quality recalibration. All RNAseq read alignments from each conceptus were then pooled for SNP calling. GATK UnifiedGenotyper was used to call variants using the following loose criteria: -stand_call_conf 0 -stand_emit_conf $0-m b q 20-$ output_mode EMIT_ALL_CONFIDENT_SITES-UALLOW_N_CIGAR_READS. The last step of these analyses was to apply a series of stringent filtering steps to remove potential false positive variants. Specifically, variants were required to have quality Q > 20 and all variants that were only found at the end ( $<6 \mathrm{bp}$ to read ends) of aligned reads were discarded. Further, RNA variants in repetitive regions annotated by UCSC (http://hgdownload.cse.ucsc.edu/goldenPath/bosTau6/database/) were discarded and variants that were in a homopolymer runs $\geq 5$ bp were also removed. In addition, intronic variants that were within $4 \mathrm{bp}$ of splice junctions were discarded. Finally, to ensure that the reads supporting the variants were uniquely aligned, BLAT (version: 34; Kent, 2002) was used to re-align these reads to the reference genome. A read alignment was required to meet two criteria to be considered as a unique mapping: i) the best alignment hit overlapped the variant site, and ii) the second best hit resulted in a $<95 \%$ sequence identity to the reference genome. Only the variants for which at least half of the alignments were unique to the reference genome were retained for further analyses.

To minimize potential false positives, only the RNA variants identified by both the HISAT2 and BWA alignment strategies were used for further analyses. Further, the 
RNA variants that were supported by the $B$. $t$. indicus DNAseq reads and/or were present in RefSeq and Ensembl dbSNP (downloaded from RefSeq and Ensembl FTP sites) were defined as genomic variants, while others were defined as RDDs. It should be noted that if a SNP in dbSNP was submitted with molecular type as "cDNA", this SNP was considered to be an RDD (i.e., a potential RNA editing site). The genomic variants were considered to be the result of SNPs and were used to assign RNAseq reads to parental alleles for allele-specific gene expression analyses, while the RDDs were used to identify A-to-I RNA editing sites. Finally, the transition/transversion (Ts/Tv) is the number of transition variants (purine-to-purine, pyrimidine-to-pyrimidine) to the number of transversion variants (purine-to-pyrimidine, pyrimidine-to-purine).

Identification of A-to-I RNA editing sites using the RNAseq data of B. t. indicus $\times$

\section{B. t. taurus $F_{1}$ conceptuses}

The RDDs were subjected to further filtering to identify A-to-I RNA editing sites. Only the RDDs that were detected in the same tissue for all four conceptuses $(\geq 10$ reads coverage/conceptus) were retained for further analyses. The rationale for this was that true A-to-I RNA editing sites should be present in all animals while low frequency SNPs should not (Ramaswami et al., 2013). It should be noted that the RNAseq reads for brain, liver, and skeletal muscle of Control \#2 were of $50 \mathrm{bp}$ in length and for these samples, the RDDs were kept for further analyses if they were present in Control \#1, \#3, and \#4 conceptuses (all 100 bp reads). Finally, the RDDs were manually annotated to determine the variant type (e.g., $\mathrm{A}>\mathrm{G}$ or $\mathrm{T}>\mathrm{C}$ ) according to the DNA strand of the transcript template. For example, if a " $\mathrm{T}>\mathrm{C}$ " variant was in a transcript that was transcribed from the antisense DNA strand then the variant type was converted from " $T>C$ " to " $A>G$ ". 
Only the RDDs that had the " $\mathrm{A}>\mathrm{G}$ " variant type were considered candidate A-to-I RNA editing sites.

To determine whether any of the A-to-I RNA editing sites identified in bovine day 105 conceptuses were conserved in human and/or mouse, $40 \mathrm{bp}$ upstream and downstream sequences (Pinto et al., 2014) of the bovine A-to-I RNA editing site were BLAST against the human (hg19) and mouse builds (mm9). Both human and mouse genomes were downloaded from the UCSC database (http://hgdownload.soe.ucsc.edu/goldenPath/hg19/bigZips/; http://hgdownload.soe.ucsc.edu/goldenPath/mm9/bigZips/, respectively). For a site to be considered to be conserved between bovine, mouse, and/or human, 70 of the 81 nucleotides had to provide a perfect match (Pinto et al., 2014) and the site had to have previously been reported to be an A-to-I RNA editing site. The previously identified editing sites in human and mouse were downloaded from the RADAR database (http://rnaedit.com; Ramaswami and Li, 2014).

Allele-specific gene expression analyses using the RNAseq data of B. t. indicus $\times$ B. t. taurus $F_{1}$ conceptuses

The DNA variants of the B. t. indicus sire were used to determine the allele-origin of the RNAseq reads in the B.t. indicus $\times$ B. t. taurus $\mathrm{F}_{1}$ hybrids. As illustrated in Fig. S5, only SNP sites that were homozygous in the $B$. t. indicus sire and for which the SNP was heterozygous or an alternate homozygote in the conceptuses were used to determine the allele-origin of the RNAseq reads. Following the allele-origin assignment, allelespecific read counts for SNPs in the same gene were aggregated for allele-specific gene 
expression analyses, as this strategy has been shown to improve the sensitivity of prediction of imprinted genes using RNAseq (DeVeale et al., 2012). To identify genes with monoallelic expression in each tissue type, allele-specific read counts of the same gene were pooled for all four individuals and the significance of allelic expression bias was determined using the binomial test (see statistical analyses section). To further filter potential false positives, these candidate genes were manually inspected using the Integrative Genomic Viewer (IGV; Robinson et al., 2011) and genes with spurious alignment of the RNAseq reads or discordant allelic bias of the SNPs within the same exon were discarded.

Validation of the A-to-I RNA editing sites in B. t. indicus $\times$ B. t. taurus $F_{1}$ hybrids

DNA and cDNA of the B. t. indicus $\times$ B. t. taurus $\mathrm{F}_{1}$ conceptuses were amplified by PCR and were subjected to Sanger sequencing to validate the A-to-I RNA editing sites identified by RNAseq. Nucleic acid isolation, cDNA synthesis, and PCR amplification were as previously reported (Chen et al., 2015; Chen et al., 2013). Primer information may be found in Table S7 (link to Table S7:

http://www.tandfonline.com/doi/full/10.1080/15592294.2016.1184805).

Validation of the allele-specific gene expression in B. t. indicus $\times$ B. t. taurus $F_{1}$ hybrids

To validate the allele specificity of the monoallelically expressed genes, both DNA and cDNA of the $\mathrm{F}_{1}$ hybrids and DNA of the sire were amplified by PCR and sequenced over the SNP sites used to ascribe parental origin of the transcripts. Nucleic 
acid isolation, cDNA synthesis, and PCR amplifications were as previously reported (Chen et al., 2015; Chen et al., 2013). Primer information may be found in Table S7.

Corroboration of the allele-specific gene expression in day 86 B. t. taurus $\times$ B. t. taurus conceptuses

B. t. taurus females (i.e., Holstein breed) were subjected to estrous synchronization and artificial insemination with sex-sorted semen from a single B. $t$. taurus bull (i.e., Holstein breed; 507HO10723 DE-SU 527 SPUR-ET). Nine B. t. taurus $\times$ B. t. taurus female conceptuses were collected at day 86 of gestation. At collection, $1 \times$ $1 \mathrm{~mm}^{2}$ samples of internal organs including brain, kidney, liver, skeletal muscle and fetal placenta (intercotyledon area) were snap frozen in liquid nitrogen and stored at $-80^{\circ} \mathrm{C}$. Both DNA and cDNA of the B.t.taurus $\times$ B. t. taurus conceptuses and DNA of the B. $t$. taurus sire were amplified by PCR to corroborate the parental-specific gene expression (i.e., imprinted status) of the identified monoallelically expressed genes detected in the $B$. t. indicus $\times$ B. t. taurus $\mathrm{F}_{1}$ hybrids. Nucleic acid isolation, cDNA synthesis, and PCR amplification were as previously reported (Chen et al., 2015; Chen et al., 2013). Primer information may be found in Table S7.

\section{DNA methylation analysis at GNAS/GNASXL domain}

Bisulfite mutagenesis of genomic DNA was performed following the One-Step Modification Procedure of the Imprint DNA Modification Kit (Sigma). Methyl Primer Express Software (version 1.0, Applied Biosystems) was used to design primers that amplify regions proximal to the GNAS and GNASXL promoters. Both amplicons spanned SNPs identified by the whole genome sequencing of the B. t. indicus sire. Bisulfite PCR 
reactions were as previously reported (Chen et al., 2015; Chen et al., 2013). The cycling programs were as follows: initial denaturation at $94^{\circ} \mathrm{C}$ for 2 minutes and 15 seconds, 45 cycles of $94^{\circ} \mathrm{C}$ for 30 seconds, $56.1 / 58.7^{\circ} \mathrm{C}$ for 45 seconds (ramping rate: $1^{\circ} \mathrm{C} / \mathrm{second}$ ), and $72^{\circ} \mathrm{C}$ for 45 seconds, and final extension at $72^{\circ} \mathrm{C}$ for 5 minutes. The PCR products were resolved by agarose gel electrophoresis and isolated as previously described (Chen et al., 2013). The PCR amplicons were cloned using pGEM-T Easy Vector System (Promega) according to the manufacturer's instructions. Bacteria used were NEB 5-alpha F'I $^{\mathrm{q}}$ Competent $E$. coli (NEB). Bacteria colonies were screened by blue-white screen method and positive colonies were subject to Sanger sequencing as described above.

\section{Statistical analyses}

All RNAseq libraries were normalized by the trimmed mean of M-values method (Robinson and Oshlack, 2010). For each gene, a binomial exact test was applied to examine if the B.t. indicus or B.t.taurus allele represented more than $85 \%$ of all reads (Chen et al., 2015). Genes were then ranked by their $p$-values and adjusted to compute false discovery rates (FDRs) using the Storey method (Storey, 2002). Genes for which the FDR was less than 0.05 were considered to be monoallelically expressed.

\section{Data access}

The DNAseq reads from the B. t. indicus sire and the RNAseq reads from the B.t. indicus $\times$ B.t.taurus $\mathrm{F}_{1}$ conceptuses are available in the NCBI Gene Expression Omnibus (GEO) database (http://www.ncbi.nlm.nih.gov/geo/) under accession numbers GSE77389 (DNAseq reads of the sire and RNAseq reads of the placenta) and GSE63509 (RNAseq reads of the brain, kidney, liver, and skeletal muscle). The sequences of the 
unannotated transcripts identified in this study have been submitted to GenBank (http://www.ncbi.nlm.nih.gov/genbank/) under accession numbers KU530101KU530107.

\subsection{Acknowledgements}

We thank Dr. Alan Ealy for providing the CT1 cell line as a kind gift. We thank Dr. Robert Schnabel for querying the SNP information of Holstein cattle. We thank Dr. Jonathan Green and Dr. Kevin Wells for helpful discussions regarding the CT1 cells. We also thank Dr. Jerry Taylor for critical reading and commenting on the manuscript. This work was supported by the National Institutes of Health (Grant 5R21HD062920), the Food for the 21st Century Program at the University of Missouri, United States Department of Agriculture National Institute of Food and Agriculture (AFRI 201167015-30688). 


\subsection{Supplemental information for Chapter 4}

\section{Supplemental figures and tables for Chapter 4}

A

The B. t. indicus sire DNAseq summary

\begin{tabular}{cccccc}
\hline Sample & Raw read pairs & Trimmed read pairs & Aligned read pairs & Uniquely aligned read pairs & $\%$ of uniquely aligned \\
B. t. indicus & $347,337,917$ & $322,794,092$ & $299,563,477$ & $230,468,801$ & 71.4 \\
\hline
\end{tabular}

DNAseq read pairs ( $2 \times 100 \mathrm{bp}$ paired-end reads) were aligned to bovine reference genome UMD3.1 using Bowtie 2 .

B

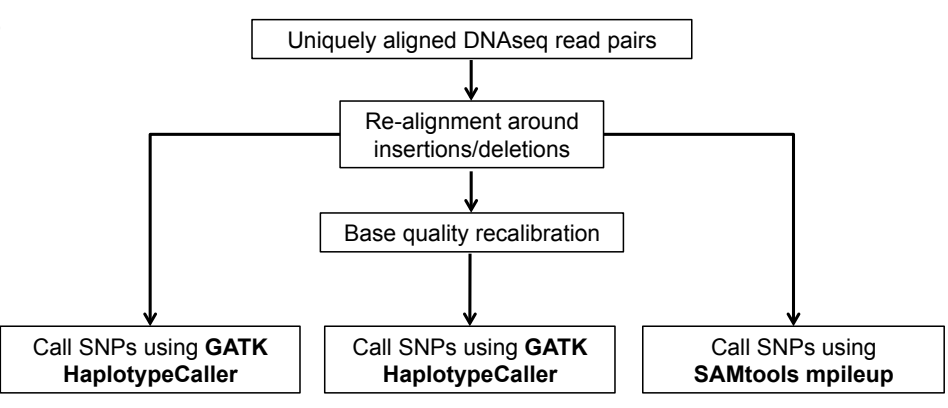

"GATK (no recalibration)"

"GATK (recalibration)"

"SAMtools (no recalibration)"

C

GATK (recalibration)

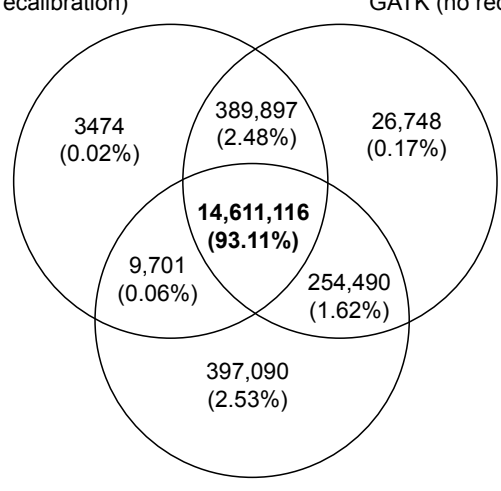

SAMtools (no recalibration)

D

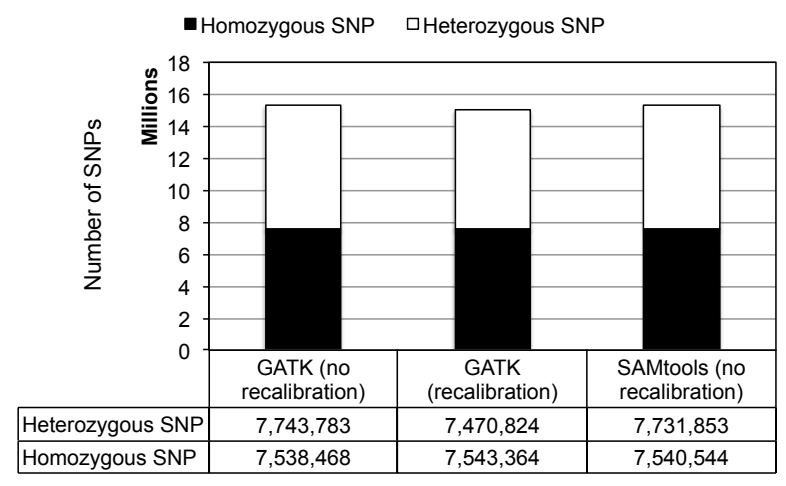

Figure S1. Genotyping the B. t. indicus sire using whole genome sequencing. A). Summary of the B. $t$. indicus sire DNAseq results. B). Pipelines used for SNP identification. C). Venn diagram showing the overlapping of the SNPs identified using different pipelines. D). Number of heterozygous and homozygous SNPs identified in the $B$. $t$. indicus sire using different pipelines. 


\section{A}
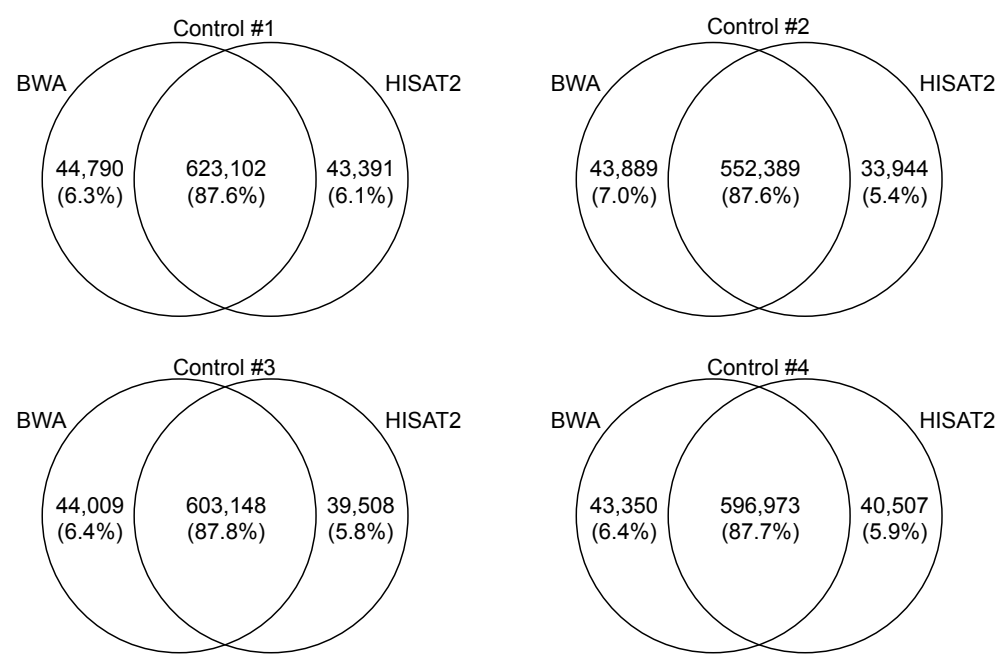

B

\begin{tabular}{cccccc} 
& Total RNA & \multicolumn{2}{c}{ RNA variants used for allele-specific expression analyses (genomic variants) } \\
\cline { 3 - 5 } Conceptuses & $\begin{array}{c}\text { Variants supported by the } \\
\text { variants }\end{array}$ & $\begin{array}{c}\text { Variants only present in } \\
\text { dbSNP (\%) }\end{array}$ & $\begin{array}{c}\text { Variants only supported by } \\
\text { the sire DNAseq reads (\%) }\end{array}$ & $\begin{array}{c}\text { RNA-DNA } \\
\text { differences (\%) }\end{array}$ \\
\hline Control \#1 & 623,102 & $406,577(65.3)$ & $68,276(11.0)$ & $135,940(21.8)$ & $12,309(2.0)$ \\
Control \#2 & 552,389 & $364,609(66.0)$ & $59,461(10.8)$ & $119,426(21.6)$ & $8,893(1.6)$ \\
Control \#3 & 603,148 & $396,161(65.7)$ & $63,739(10.6)$ & $130,720(21.7)$ & $12,528(2.1)$ \\
Control \#4 & 596,973 & $392,030(65.7)$ & $63,251(10.6)$ & $129,604(21.7)$ & $12,088(2.0)$ \\
\hline
\end{tabular}

C Genomic variants (Ts/Tv: 2.5)

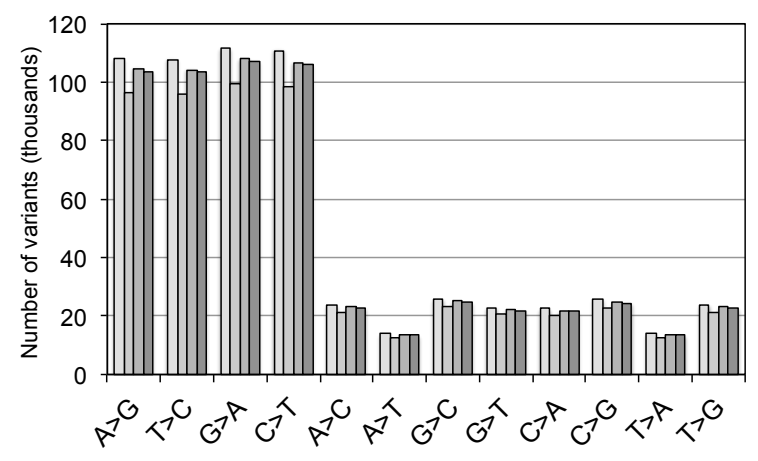

D

RNA-DNA differences (Ts/Tv: 14-18)

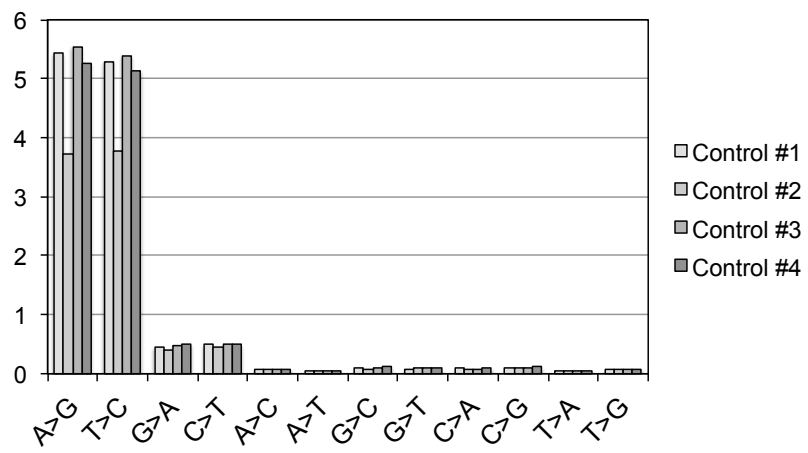

Figure S2. Genotyping the B. $t$. indicus $\times$ B. t. taurus $\mathrm{F}_{1}$ hybrids using RNAseq. A). Venn diagrams showing the overlapping of the RNA variants identified using the HISAT2 or BWA as reads aligner. B). Comparison of the identified RNA variants with bovine dbSNP entries and the $B . t$. indicus sire SNPs. C). Variant type distribution of the genomic variants (i.e., variants matched to bovine dbSNP entries and/or supported by the $B$. $t$. indicus sire DNAseq reads). D). Variant type distribution of the RNA-DNA differences (RDDs). Ts = transition (purine-to-purine, pyrimidine-topyrimidine); $\mathrm{Tv}=$ transversion (purine-to-pyrimidine, pyrimidine-to-purine). 
A

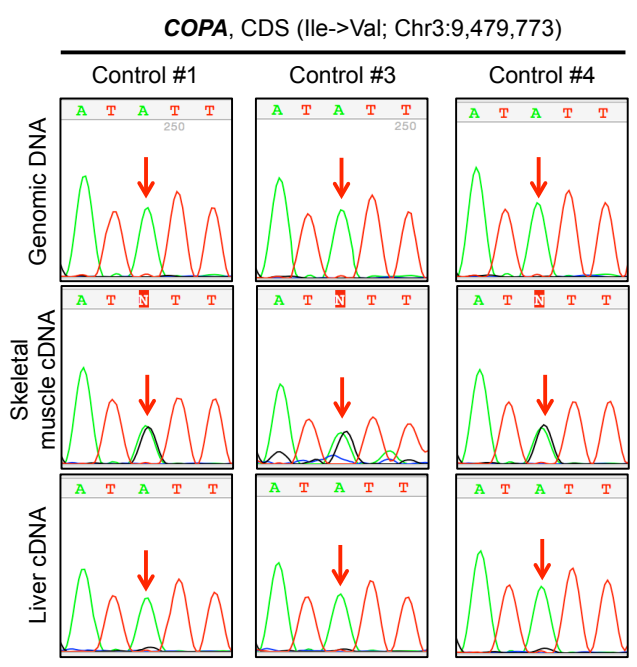

B

GRIK2, CDS (GIn->Arg; Chr9: 48,600,848) GRIK2, CDS (Gly->Gly; Chr9: 48,600,865)

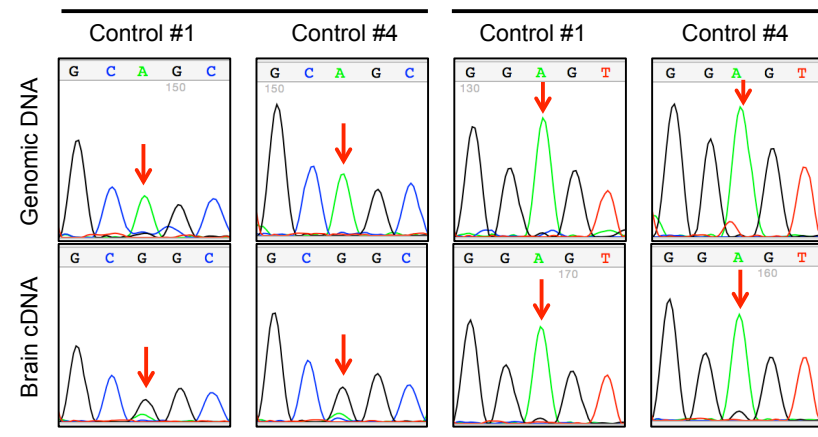

C

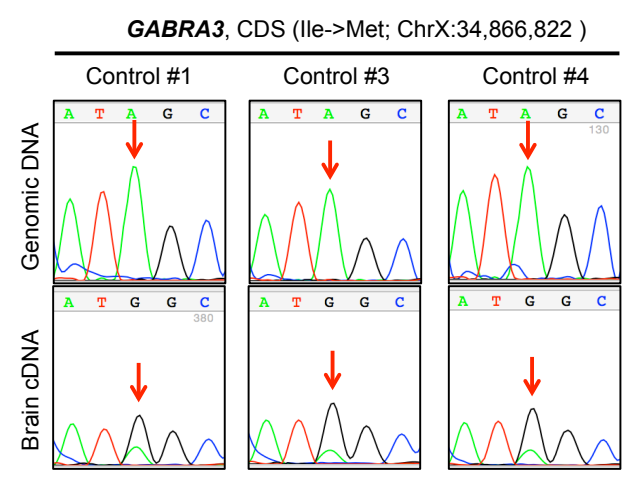

GABRA3, CDS (Asn->Asp; ChrX:34,866,869)

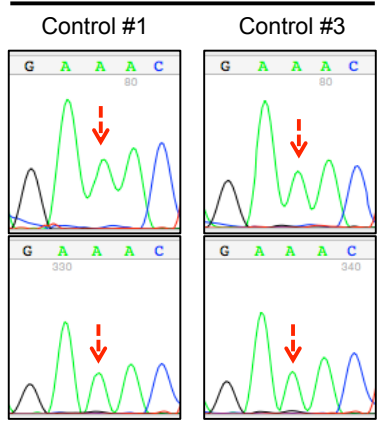

Figure S3. Validation of the identified A-to-I RNA editing sites in the B.t. indicus $\times$ B. $t$. taurus $\mathbf{F}_{1}$ hybrids by Sanger sequencing. The red arrows indicate the editing sites. A). An example of A-to-I editing in coding sequences (CDS) of COPA. B). Two RNA editing sites in CDS of GRIK2. C). Two RNA editing sites in the CDS of GABRA3, one of which was not validated by Sanger sequencing (i.e., ChrX:34,866,869). 

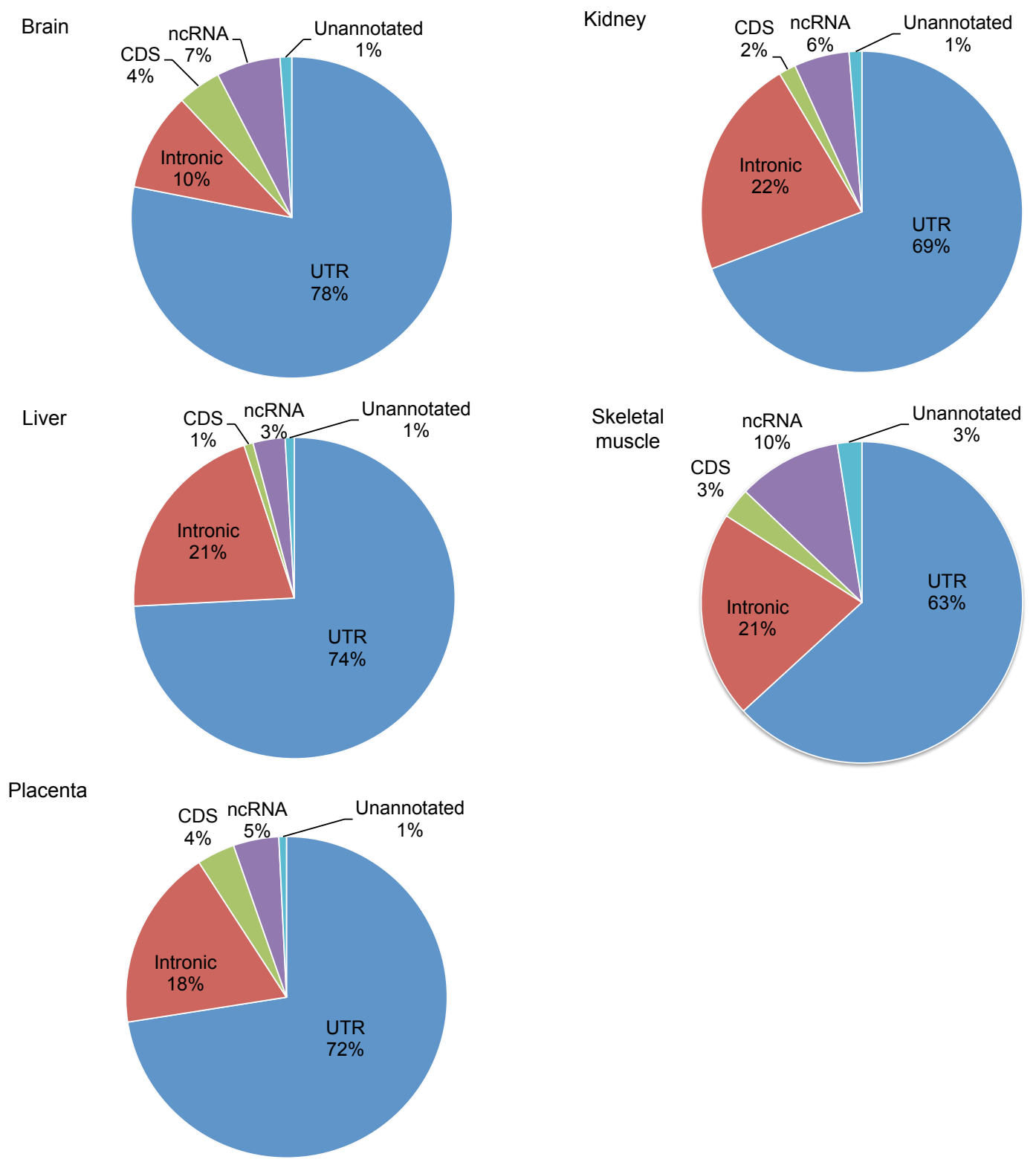

Figure S4. Pie charts for the A-to-I RNA editing sites at different gene sections in each tissue of the B. t. indicus $\times$ B. t. taurus $\mathbf{F}_{\mathbf{1}}$ hybrids. CDS: coding sequence; UTR: untranslated region; ncRNA: noncoding RNA. 


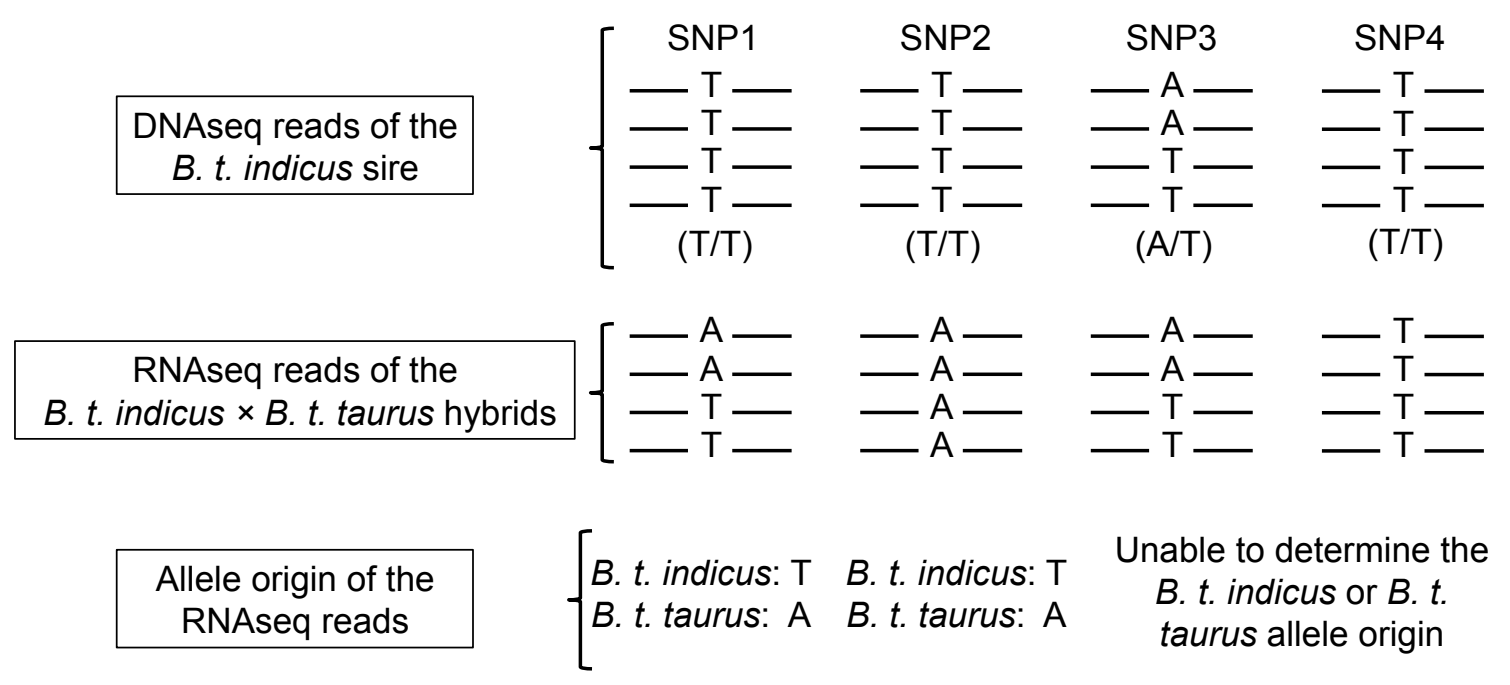

Figure S5. Illustration of the strategy used to assign parental alleles of RNAseq reads in the B. t. indicus $\times$ B. t. taurus $\mathrm{F}_{1}$ hybrids. 
A

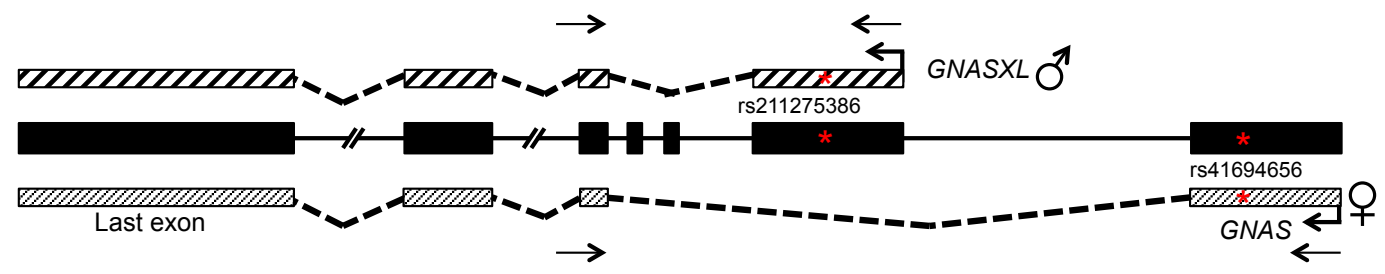

GNASXL (B. t. indicus $\times$ B. t. taurus)

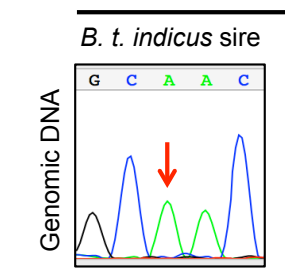

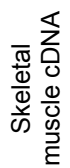

B

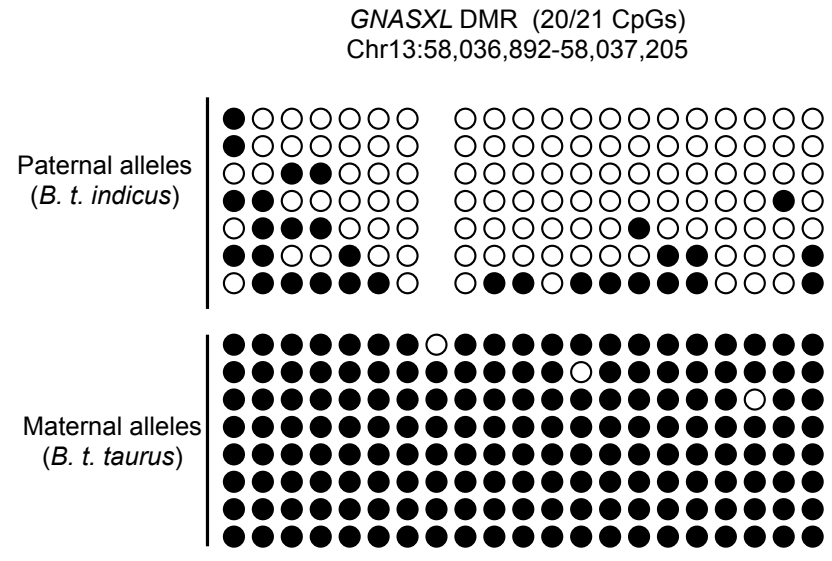

GNAS (B. t. indicus $\times$ B.t.taurus)

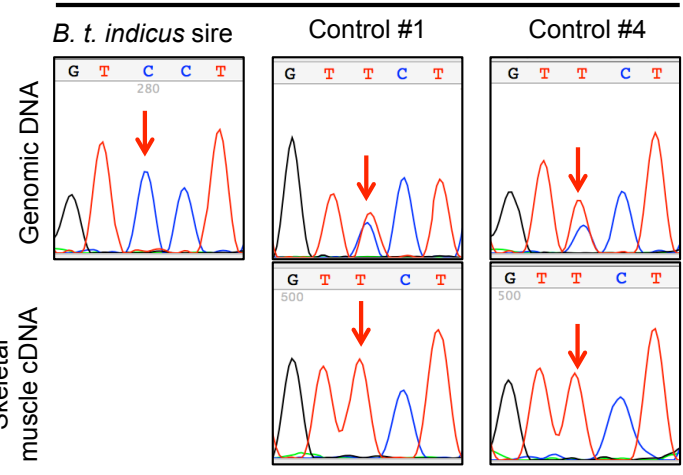

GNAS DMR (17 CpGs) Chr13:58,045,872-58,046,294

0000000090000009 O०900000989898909 00000000800000809

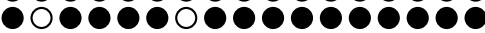

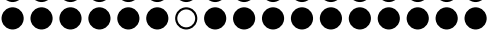

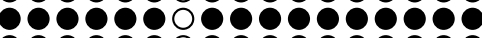

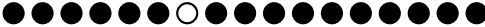

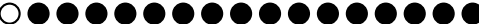

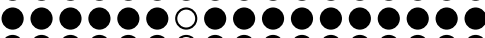
๑०000000000000000

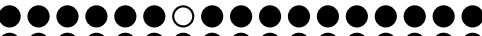


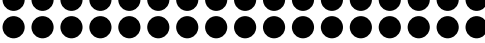

00000000000000000

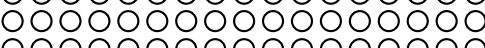
00000000000000000 00000000000000 00000000000000 - 00000000000000 89890800000000000

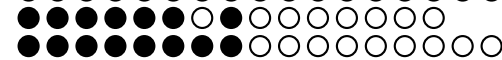

Figure S6. Allele-specific gene expression and DNA methylation analyses at $G N A S / G N A S X L$ imprinted domain in the B.t. indicus $\times$ B. t. taurus $\mathrm{F}_{1}$ hybrids. A). Schematics of $G N A S / G N A X L$ loci and Sanger sequencing validation of the allele-specific gene expression of GNAS and GNASXL. Details are as Figure 3. Primers used for RT-PCR are shown as arrows facing each other. B). Full bisulfite maps of GNAS and GANSXL DMR. Each row = one strand of DNA; open circles, unmethylated $\mathrm{CpG}$ sites; filled circle, methylated $\mathrm{CpG}$ sites; missing circles are due to SNPs or undetermined bases because of sequencing issues. 
A

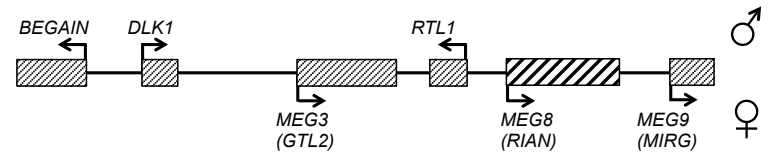

MEG8 (B.t. indicus $\times$ B. t. taurus)

MEG9 (B.t. indicus $\times$ B. t. taurus)
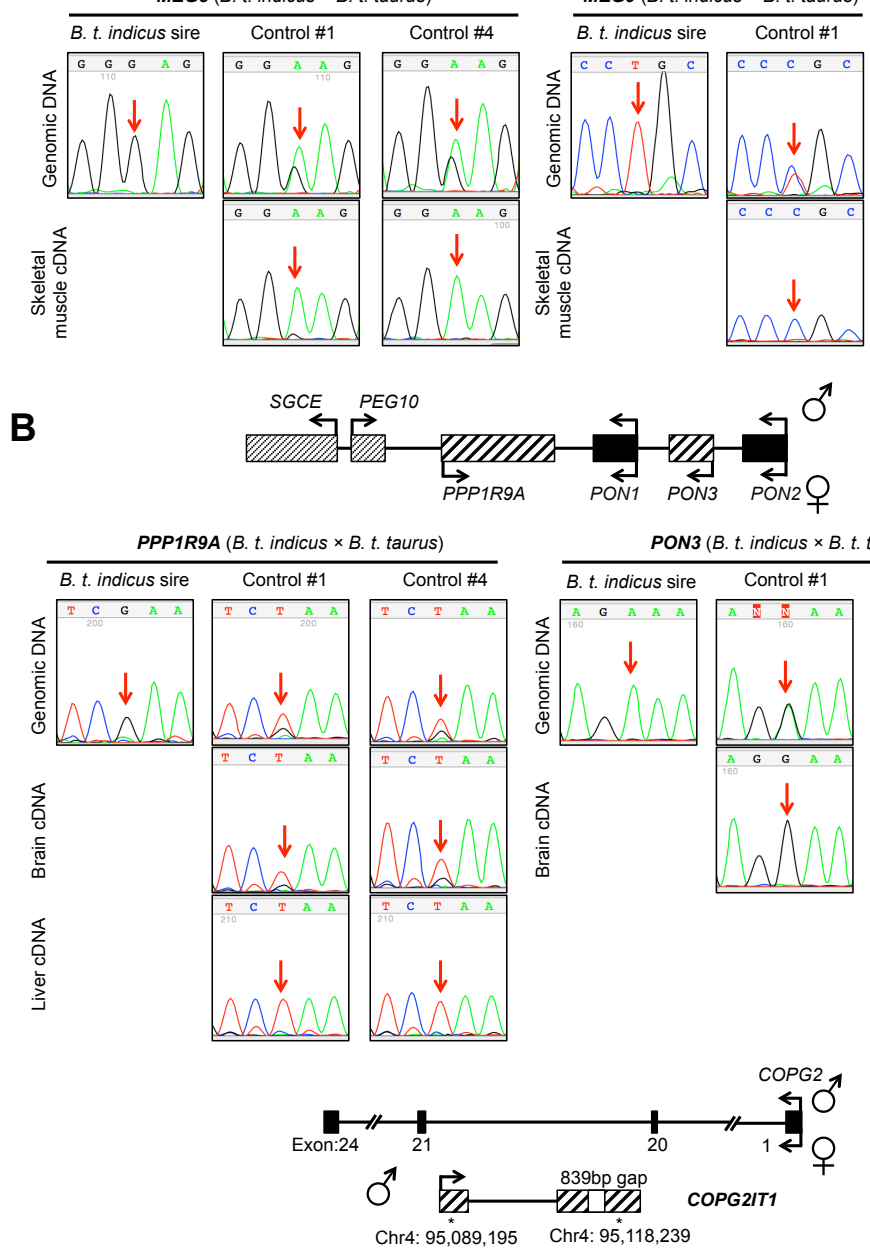

COPG2IT1 (B. t. indicus $\times$ B. t. taurus; Chr4: 95,089,195) $\quad$ COPG2IT1 (B.t. indicus $\times$ B. t. taurus; Chr4: $95,118,239$ )
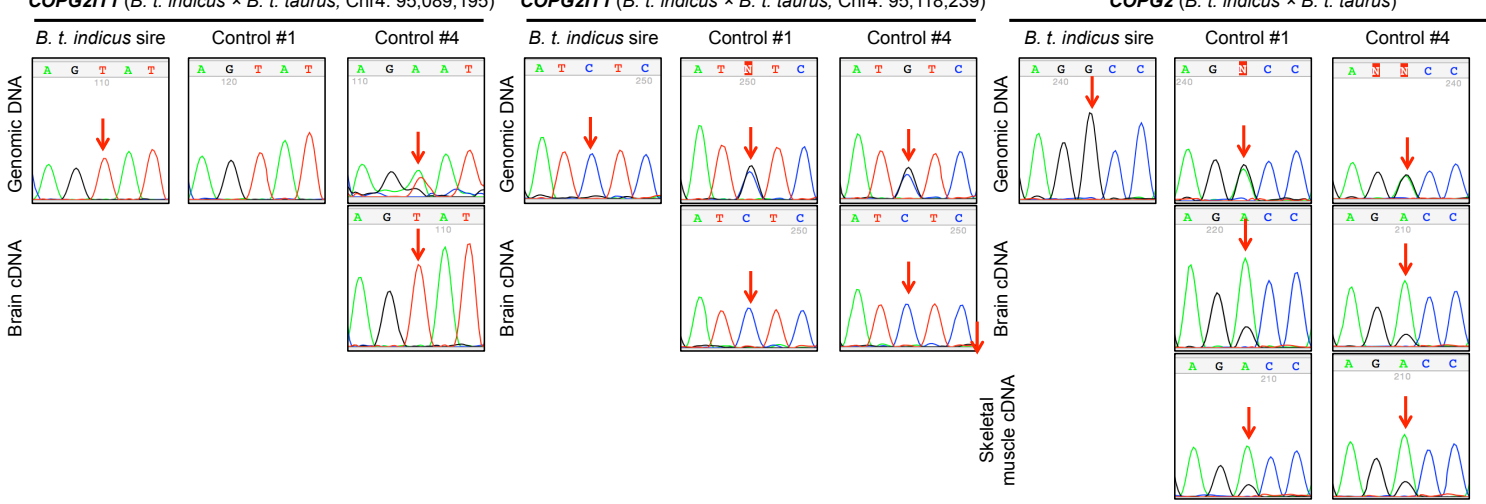

Figure S7. Validation of the allele-specific expression of the genes that are previously described as imprinted in human and/or mouse in the B.t. indicus $\times$ B. t. taurus $\mathbf{F}_{1}$ hybrids. A). GTL2/DLK1 domain. B). PEG10/SGCE domain. C). MEST/COPG2 domain. Details are as Figure 3. 

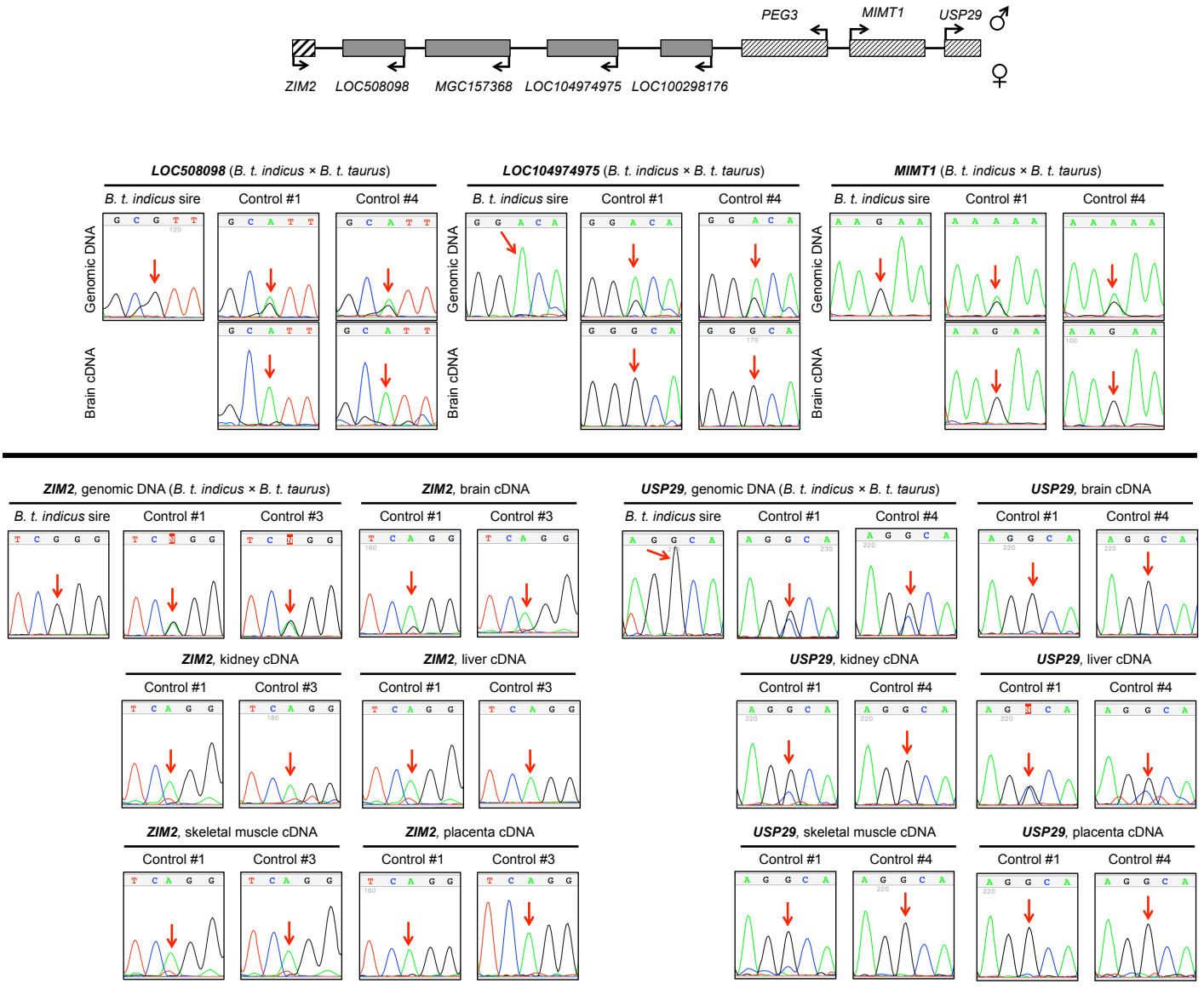

Figure S8. Validation of the allele-specific gene expression in PEG3/USP29 imprinted domain in the B. t. indicus $\times$ B. t. taurus $\mathbf{F}_{1}$ hybrids. Details are as Figure 3 . 

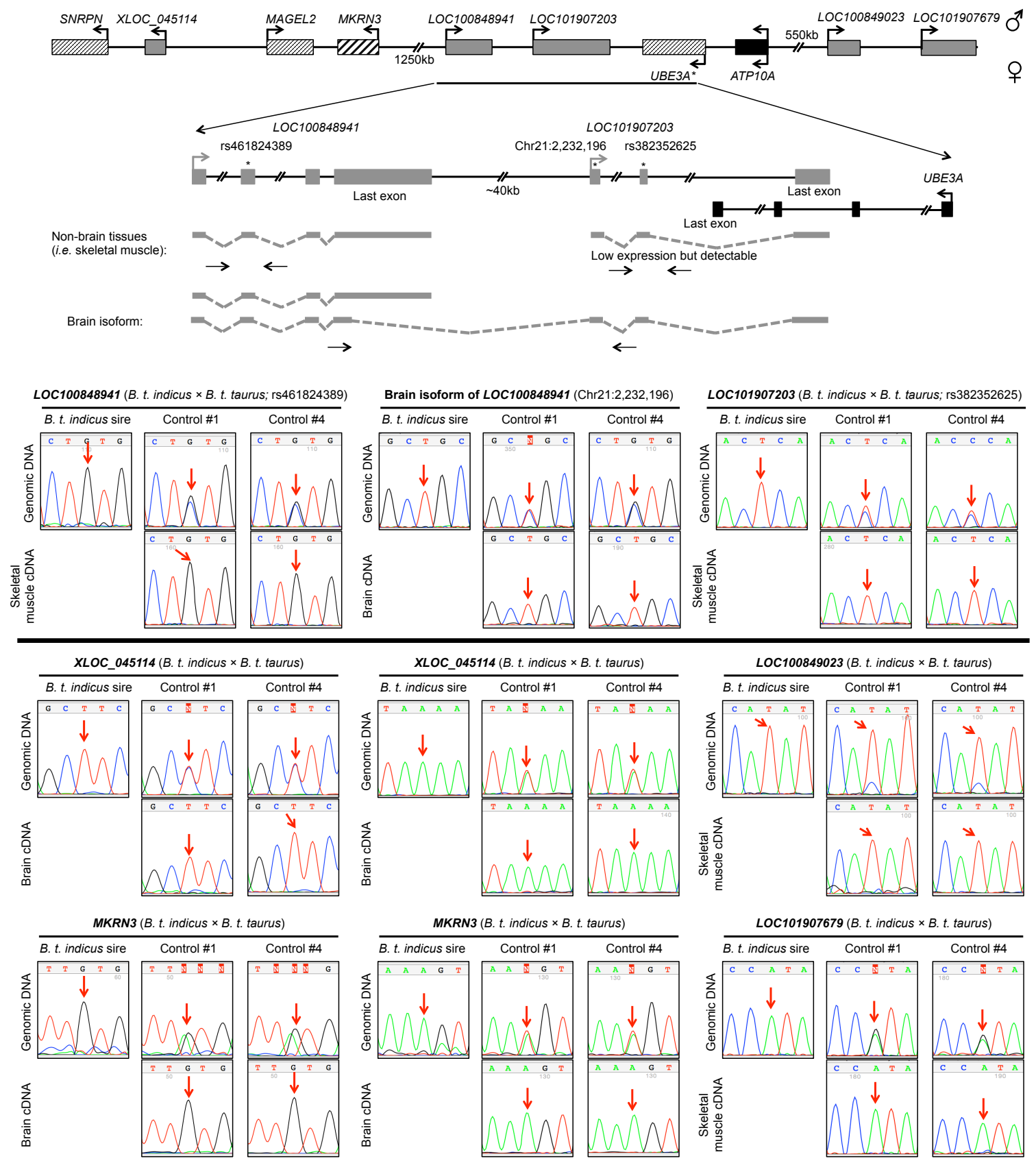

Figure S9. Validation of the allele-specific gene expression in $S N R P N$ imprinted domain in the B. t. indicus $\times$ B. t. taurus $\mathbf{F}_{1}$ hybrids. For LOC100848941 and LOC101907203, SNPs used for allelic expression analyses are indicated by asterisks. RT-PCR primers amplifying each transcript are indicated by arrows facing each other. Other details are as Figure 3. 
Figure S10.

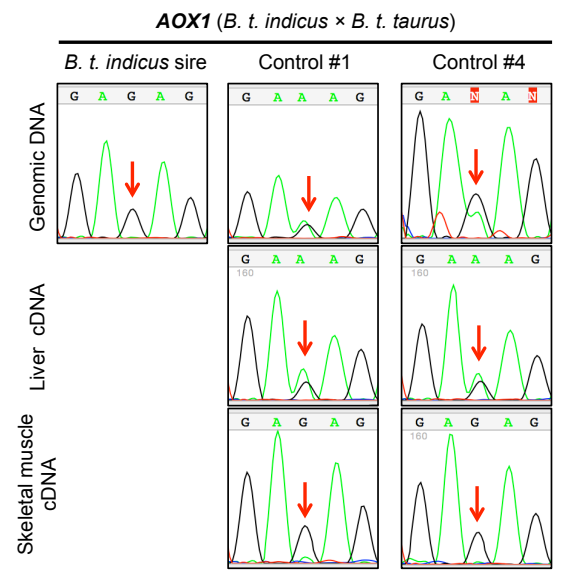

No SNPs were identified in the $B$. t. taurus $\times B$. t. taurus conceptuses
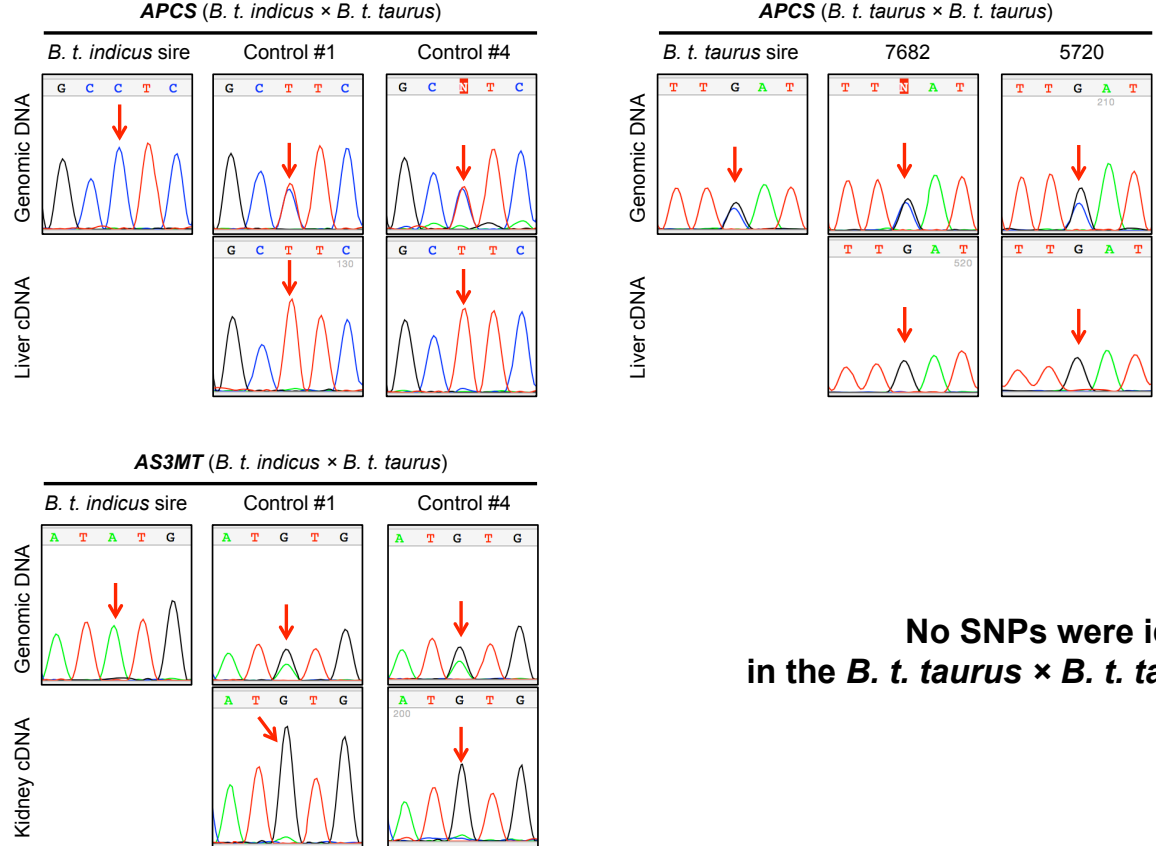

No SNPs were identified in the B.t. taurus $\times B$. t. taurus conceptuses 
Figure S10.
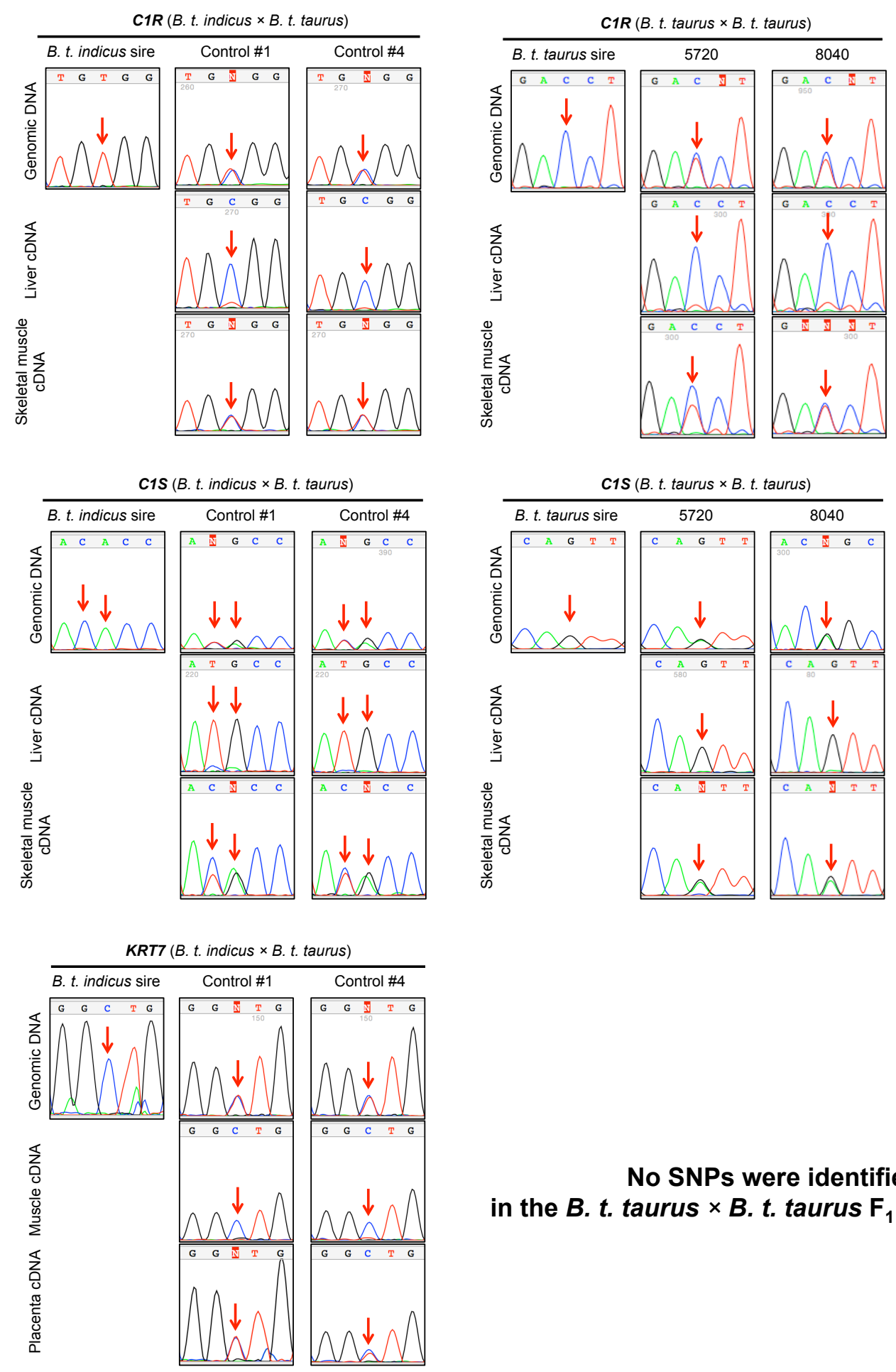

\author{
No SNPs were identified \\ in the B. t. taurus $\times$ B. t. taurus $F_{1}$ conceptuses
}


Figure S10.

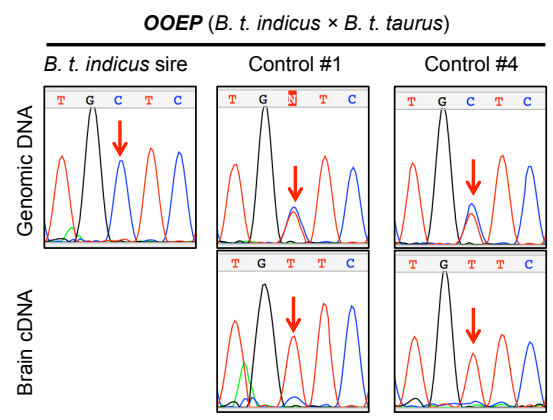

\section{No SNPs were identified in the B. t. taurus $\times$ B. t. taurus conceptuses}
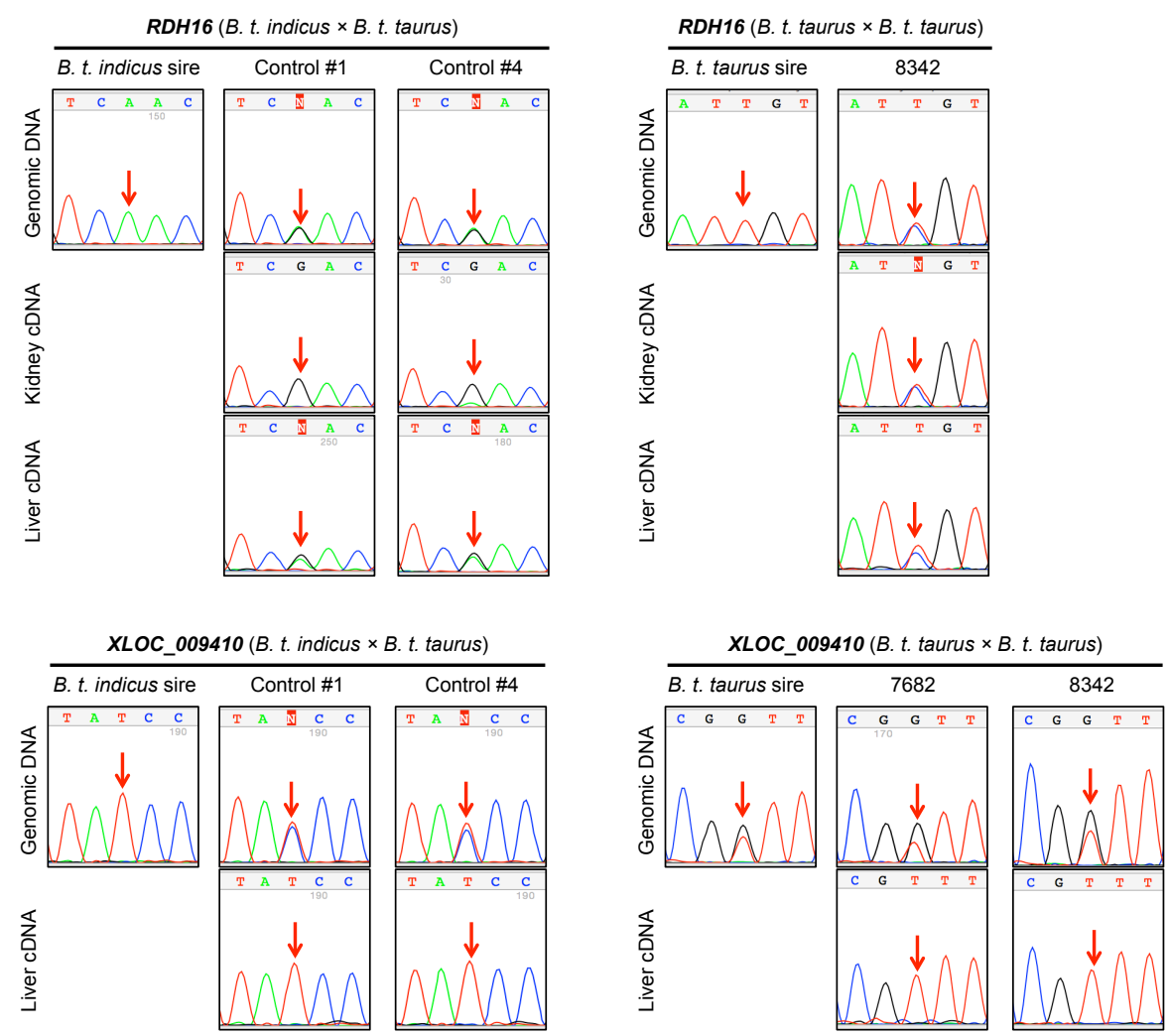
Figure S10.
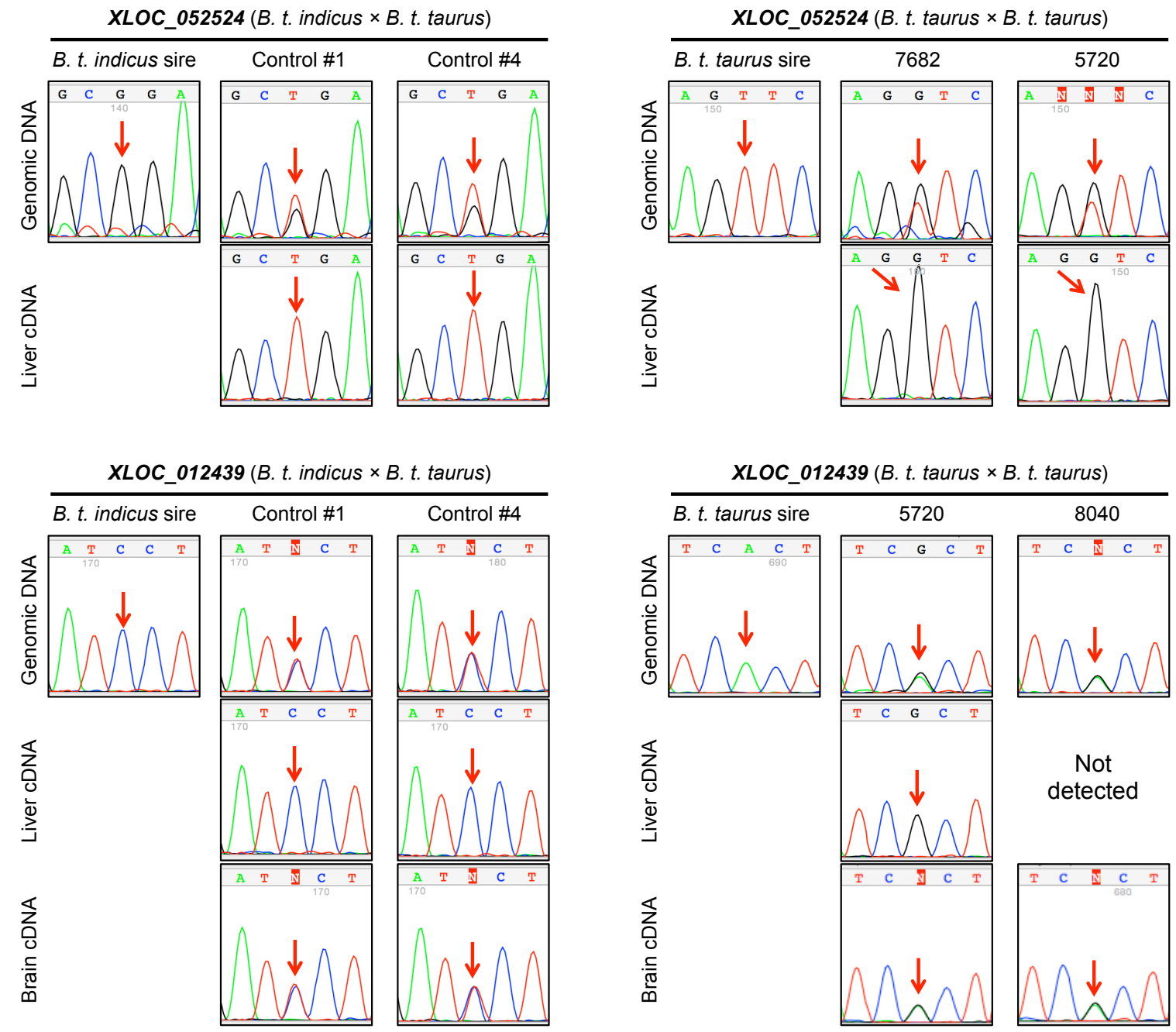

Figure S10. Corroboration of the allele-specific expression of the singleton genes identified in the B.t. indicus $\times$ B. t. taurus $\mathrm{F}_{1}$ hybrids and the B.t.taurus $\times$ B. t. taurus conceptuses. For each gene, the left panel is the Sanger sequencing validation of the monoallelic expression in the B. t. indicus $\times$ B. t. taurus $\mathrm{F}_{1}$ hybrids while the right panel is the corroboration of the allelic expression in the B.t.taurus $\times$ B.t.taurus conceptuses. Other details are as in Figure. 4 . 
Figure S11.
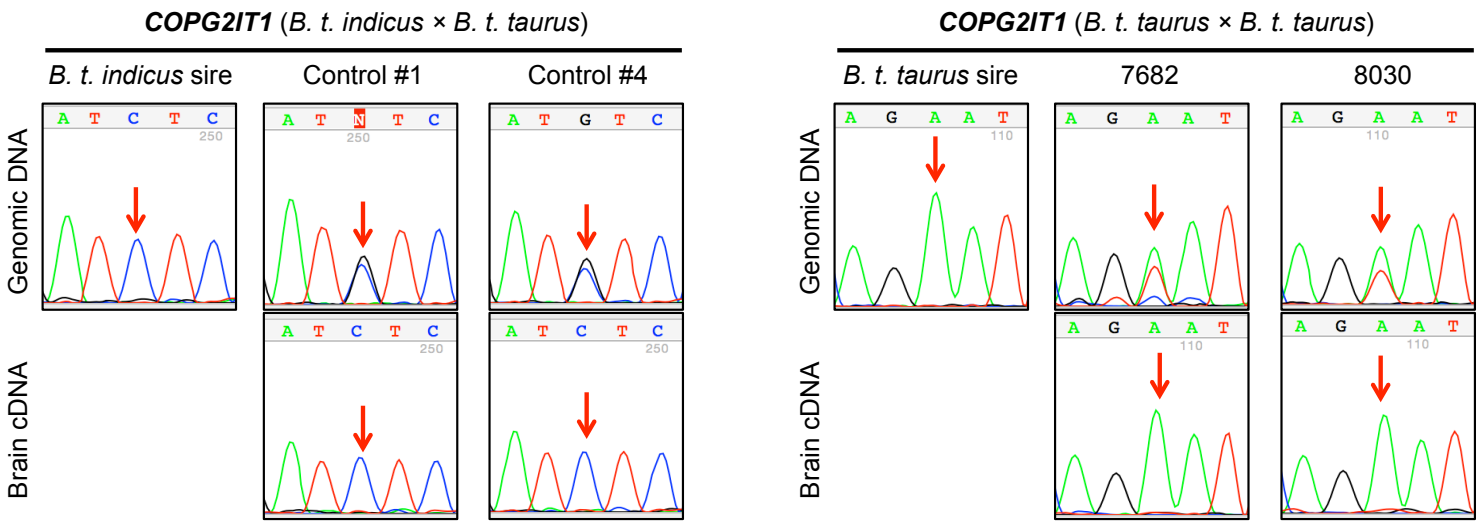

GNAS/GNASXL (B. t. indicus $\times$ B. t. taurus, skeletal muscle cDNA)

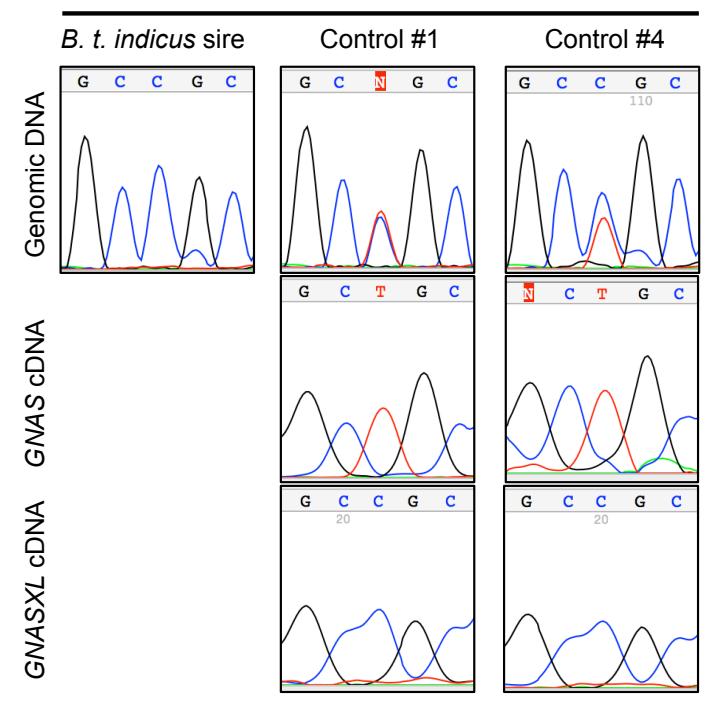

GNAS/GNASXL (B. t. taurus $\times$ B. t. taurus,

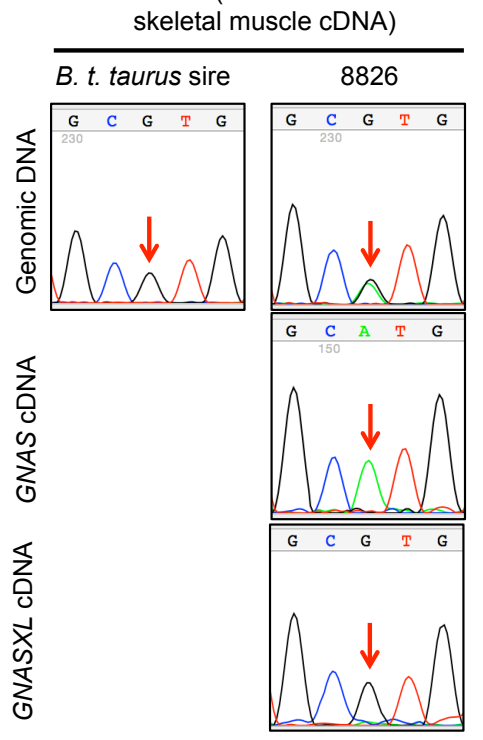

LOC508098 (B. t. indicus $\times$ B. t. taurus)

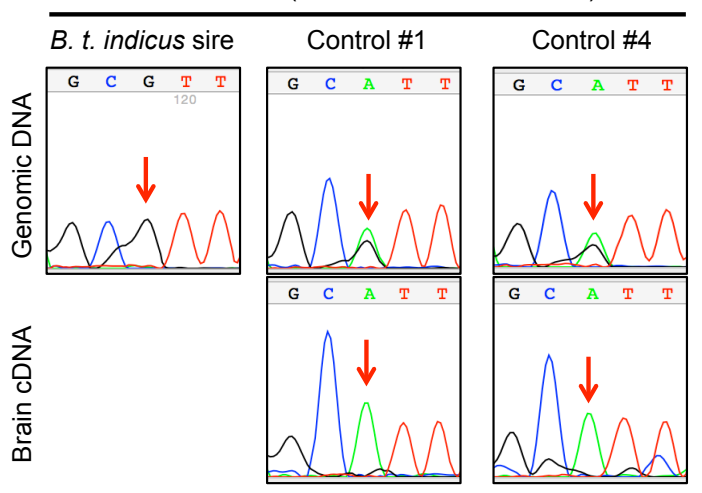

LOC100849023 (B. t. indicus $\times$ B. t. taurus)
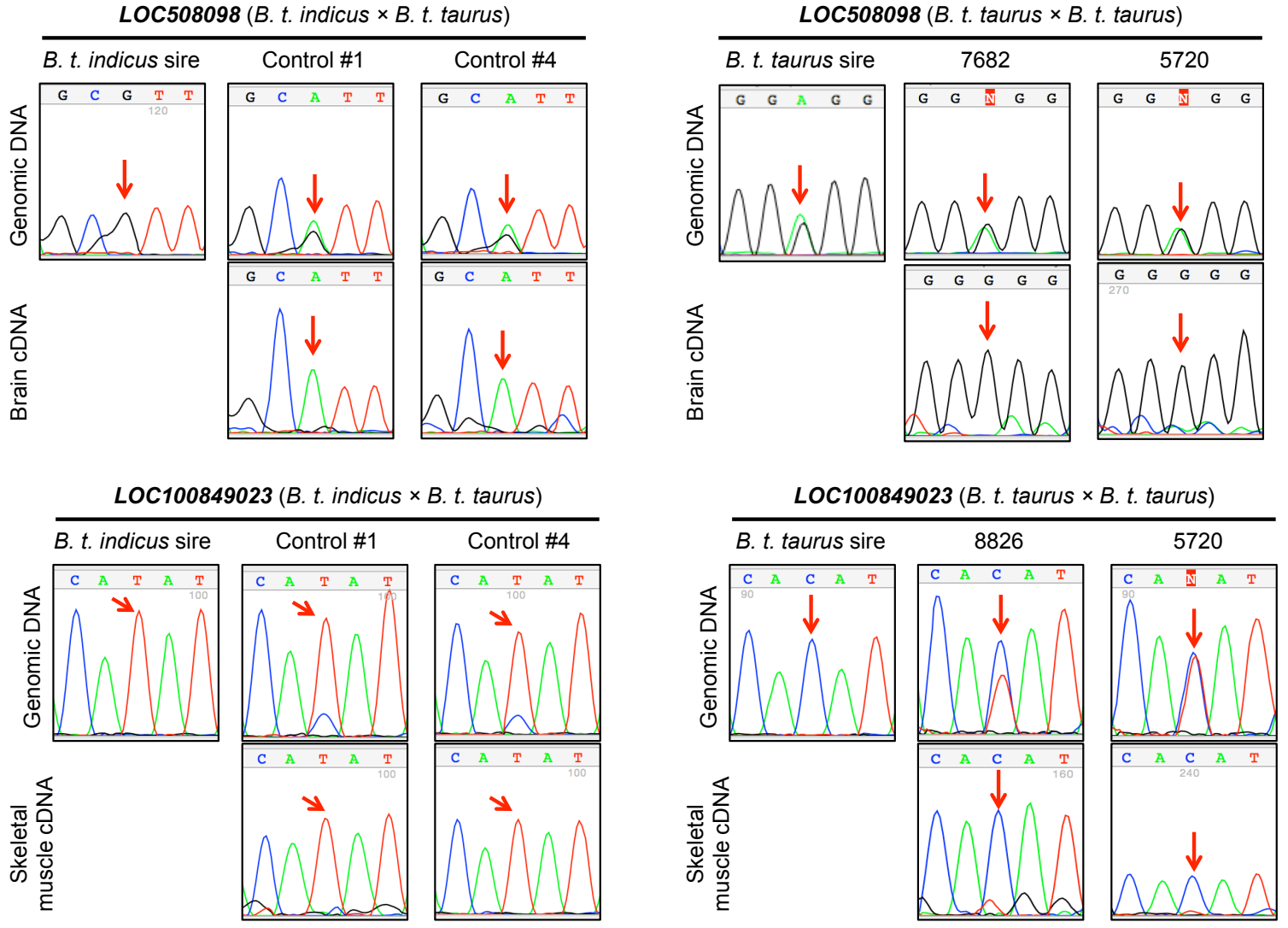
Figure S11.
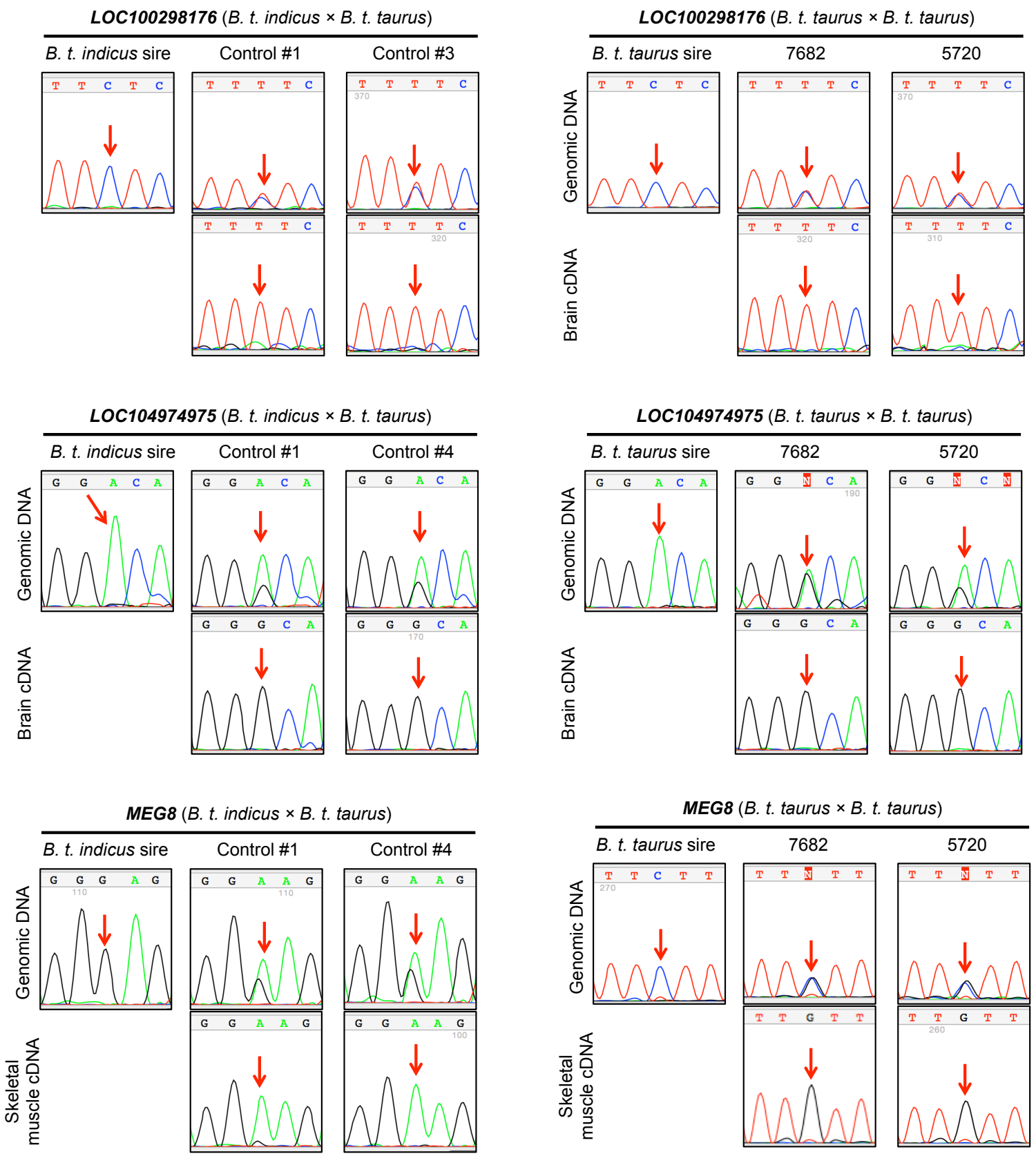
Figure S11.
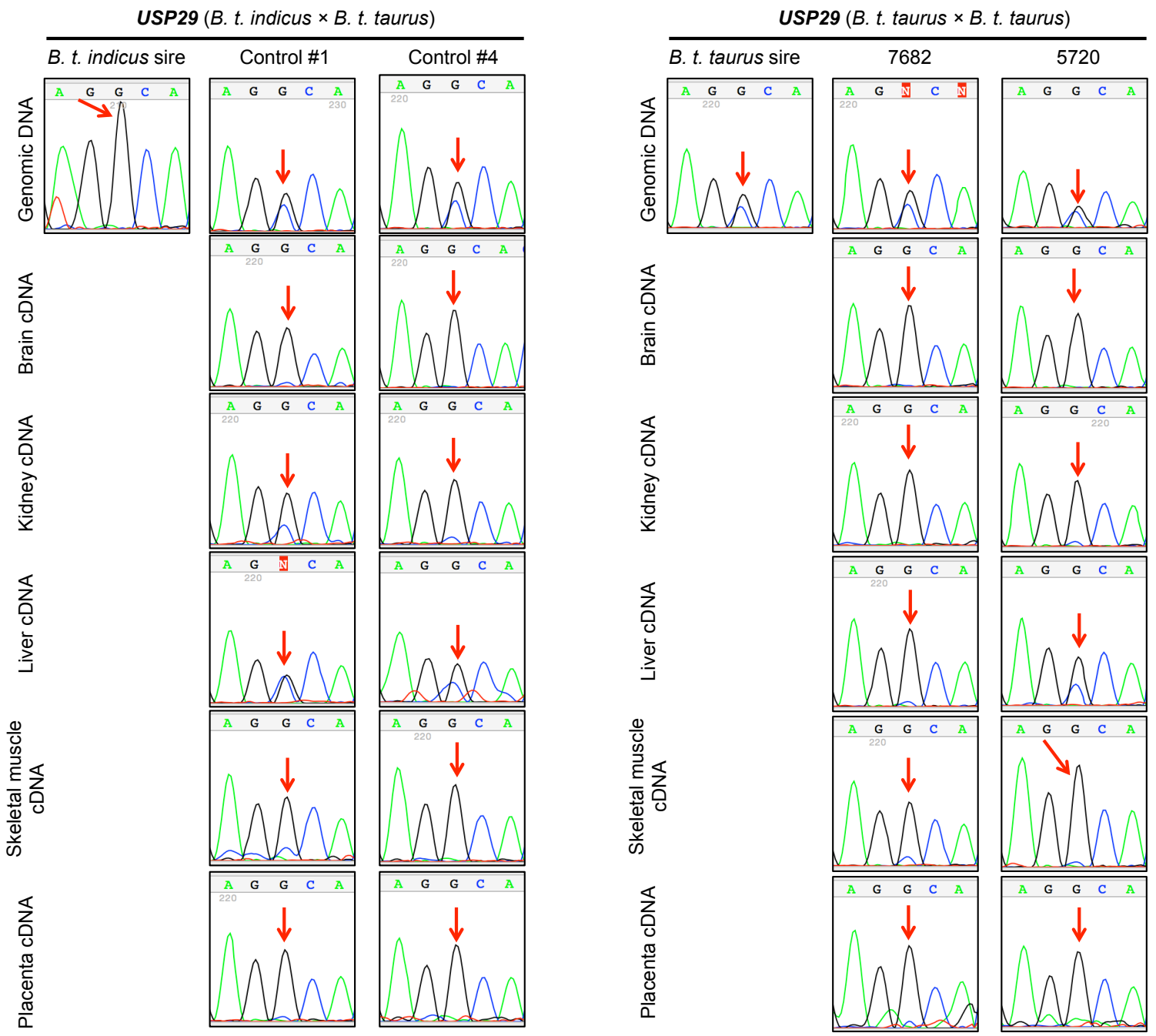
Figure S11.
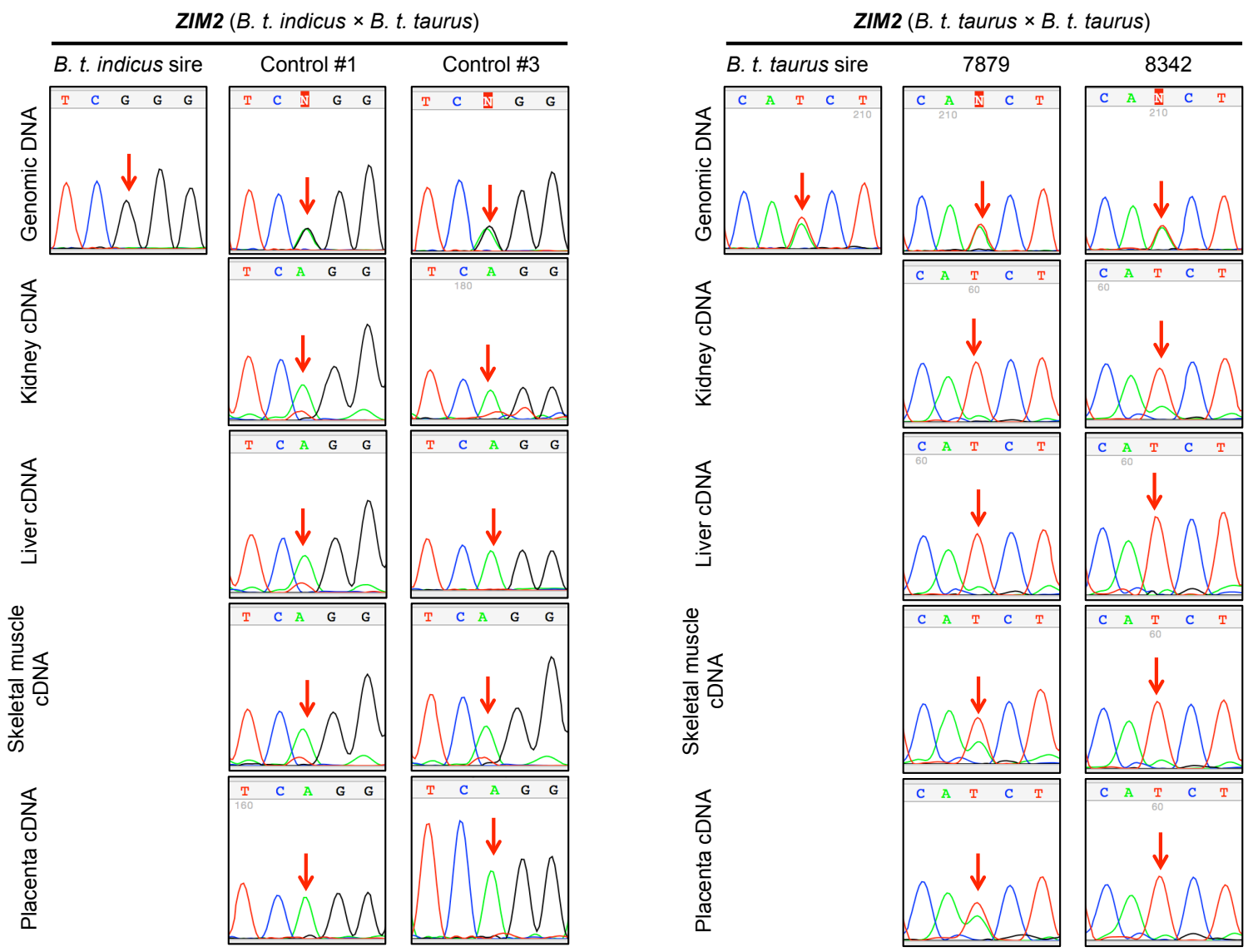

Figures S11. Corroboration of the allele-specific expression of the genes in the B.t.taurus $\times$ $B$. $t$. taurus conceptuses that are either known imprinted genes in human/mouse or are located in known imprinting clusters. Details are as Figure. 4. 


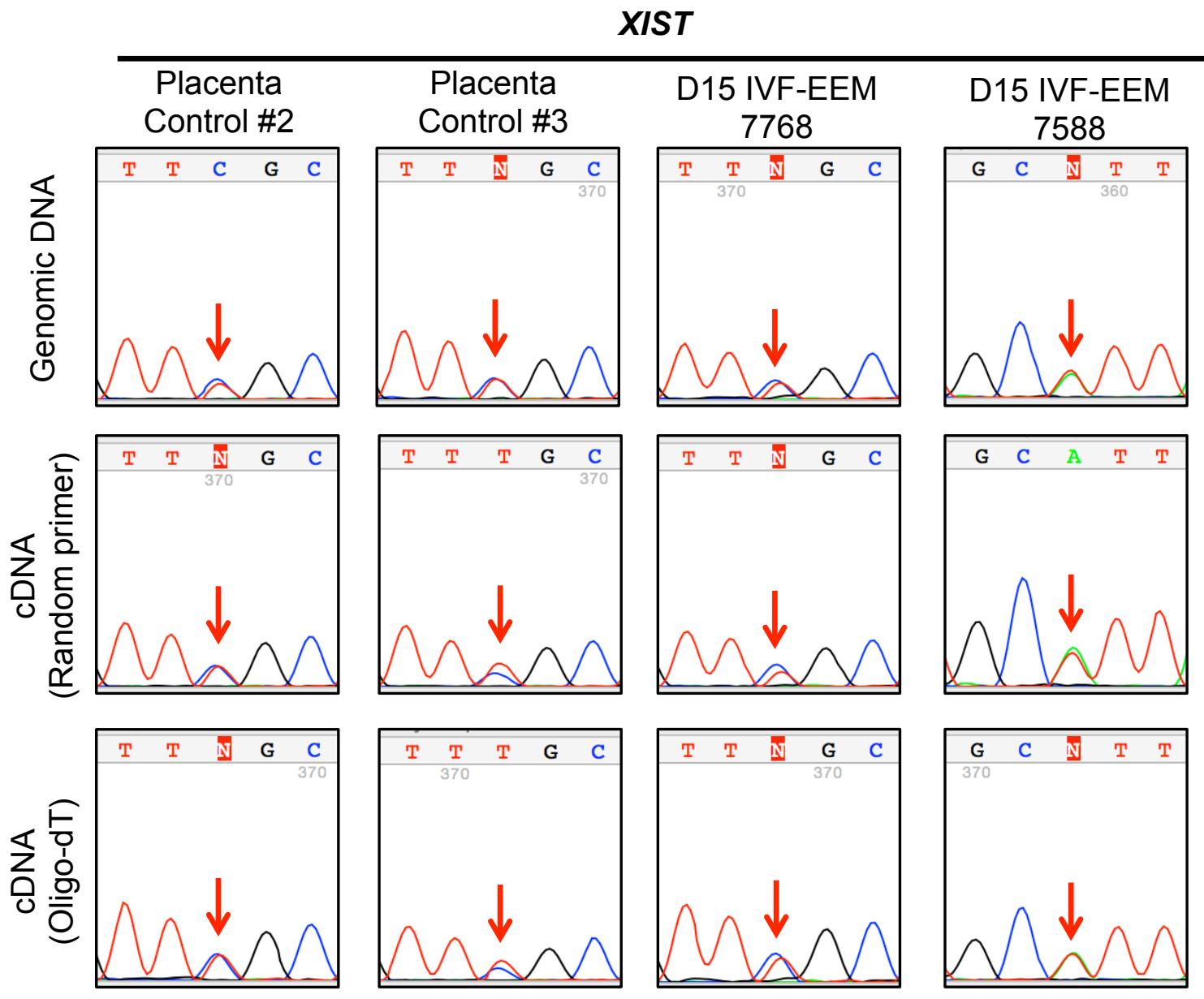

Figure S12. Allelic expression analyses of XIST. Other details are as Figure 6. 


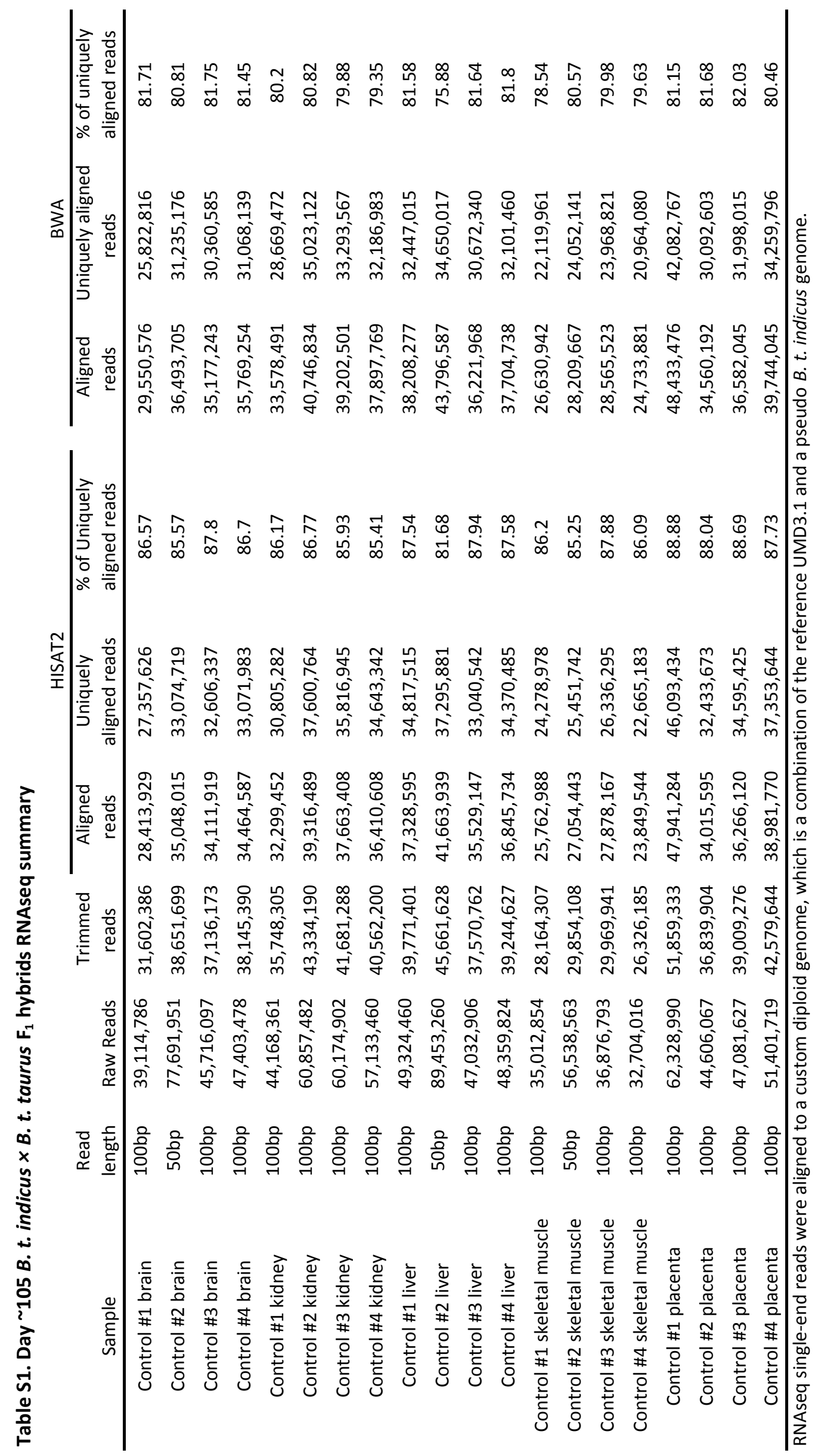




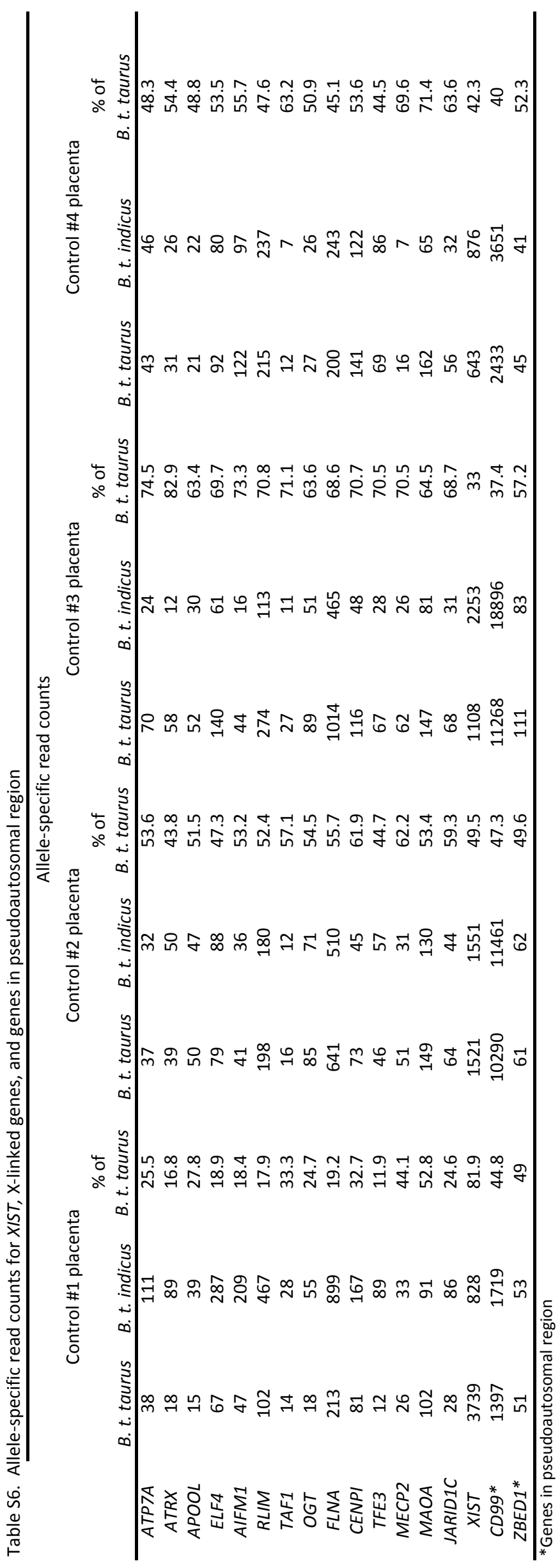




\section{Chapter 5 General discussion}

In the 1960s, Drs. Beckwith and Wiedemann first described BWS as a somatic overgrowth disorder with predisposition of childhood cancer (Beckwith, 1969;

Wiedmann, 1964). In the 1980s, genetic linkage studies indicated that BWS is related to chromosome 11p15.5 (Koufos et al., 1989; Ping et al., 1989), a region that turned out to harbor two imprinted clusters, namely, the KCNQ1 locus and the H19/IGF2 locus. Since then, with the help of several mouse models of BWS, the main focus of the field has been to address how loss-of-imprinting at $11 \mathrm{p} 15.5$ leads to the complex and variable phenotypic features of BWS. However, to date, there has been no report of molecular features that can consistently and reliably diagnose and predict BWS and the associated variable phenotypes.

The first cases of ART-induced LOS were reported in 1995 (Farin and Farin, 1995 ) and several reasons have prompted us to use LOS as an animal model to study the molecular etiology of human BWS. First, LOS recapitulates most BWS phenotypic features including the cardinal symptoms such as somatic overgrowth, enlarged tongue, ear malformation, and abdominal wall defects (Chen et al., 2013; Mussa et al., 2016; Young et al., 1998). Second, LOS fetuses exhibit the most common epimutation observed in BWS, namely, hypomethylation at the KCNQ1 locus (Chen et al., 2013; Hori et al., 2010). Third, both BWS and LOS have a higher risk in the offspring born with the use of ART and both syndromes can also occur naturally (reviewed in Choufani et al., 2010; Personal communication with RMR; Young et al., 1998). Fourth, the development of LOS in bovine is more similar to BWS compared to the mouse models, which rely on 
genetic manipulations of the imprinted genes and exhibit more severe phenotypes that are not observed in BWS (e.g., embryonic/neonatal lethality).

We have advanced the field by providing the following insights. 1) LOS is a multi-locus loss-of-imprinting syndrome and the number of genes exhibiting loss-ofimprinting is positively correlated with bodyweight. 2) Loss of allele-specific expression of imprinted genes in LOS is associated with aberrant DNA methylation at the associated imprinted DMRs in a tissue-specific manner. 3) LOS fetuses not only exhibit loss of allele-specific expression of imprinted genes, but also display differential transcript amount of these genes compared to controls. 4) Different tissues within LOS fetuses have perturbations of distinct gene pathways and in particular, multiple pathways involved in myoblast proliferation and fusion into myotubes are mis-regulated in skeletal muscle of LOS fetuses. 5) Numerous local methylation differences exist between control and LOS. 6) Most of identified DEGs between control and LOS could not be directly associated with altered DNA methylation, suggesting that caution should be exercised when making conclusions about the etiology of such syndromes by interpreting time-point-specific DNA methylation data.

In addition to characterizing the transcriptome and methylome in LOS at day $\sim 105$ pregnancy (gestation $=280$ days), we also identified eight novel bovine imprinted genes and showed that monoallelic gene expression can be the result of cis-eQTL effects. Further, during the de novo identification of novel imprinted genes, it was imperative to differentiate DNA polymorphisms from RNA editing sites in order to accurately assign the parental-origin of RNAseq read. By doing this, we identified 671 A-to-I RNA editing 
candidate sites, thus increasing the list in bovine by 651 potential sites. It should be noted that no differences in A-to-I RNA editing were observed between LOS and control.

Despite the progress accomplished by my dissertation research, the molecular etiology of BWS/LOS is still not understood and several key points remain to be addressed in future studies. The following sections will present and address some of these points.

\subsection{How does the use of ART lead to epigenetic instability?}

\subsubsection{Superovulation and loss-of-imprinting}

Superovulation is commonly used to increase oocyte number for assisted reproduction and numerous studies have reported that superovulation results in loss-ofimprinting (reviewed in Denomme and Mann, 2012). For instance, superovulation can induce gain of methylation of the paternally methylated $H 19 / I G F 2$ DMR and loss of methylation of the maternally methylated Snrpn DMR in mouse blastocysts (MarketVelker et al., 2010). However, no DNA methylation defects at imprinted gDMRs (i.e., Snrpn DMR, KvDMR1, Peg3 DMR, and H19 DMR) are detected so far in ovulated oocytes following hyperstimulation, which suggests that superovulation leads to loss-ofimprinting by affecting imprint maintenance in early embryos rather than imprint establishment in oocytes (Denomme et al., 2011).

Several maternal factors have been identified to maintain the epigenetic stability in early embryos such as the oocyte-specific DNA methyltransferase Dnmt1o and Kruppel-associated box zinc finger proteins Zfp57 and Trim28 (Howell et al., 2001; Li et 
al., 2008; Messerschmidt et al., 2012). Absence of these maternal factors in oocytes leads to embryonic or neonatal lethality largely due to the defects in genomic imprints maintenance in early embryos (Howell et al., 2001; Li et al., 2008; Messerschmidt et al., 2012). It is possible that superovulation induces ovulation of oocytes that do not reach full maturity and do not have sufficient maternal factors such as Dnmt1o, Zfp57, and Trim28 to maintain DNA methylation at imprinted gDMRs in early embryos. In addition, superovulated oocytes may represent a rather heterogeneous population of cells with some oocytes reaching full maturity while some others with insufficient maternal epigenetic factors, which may explain the stochastic feature of superovulation-induced loss-of-imprinting (i.e., only some embryos showing loss-of-imprinting while others are normal). In future studies, determination of the transcriptome of individual naturally- and superovulated oocytes will ascertain the molecular machinery that is responsible for the developmental incompetence of oocytes caused by hyperstimulation.

\subsubsection{Embryo culture and loss-of-imprinting}

Following fertilization, embryos are usually cultured in vitro to eight-cell or blastocyst stage before being transferred to a recipient. It is well established that embryo culture can result in loss-of-imprinting in the absence of superovulation (reviewed in Denomme and Mann, 2012). Notably, mouse embryos cultured in what is considered an optimal medium (i.e., KSOM) are less susceptible to loss-of-imprinting and gene expression changes compared to a suboptimal medium like Whitten's (Mann et al., 2004; Rinaudo and Schultz, 2004). It may be speculated that embryos cultured in vitro undergo a stress response that consumes more energy otherwise would be used for imprint maintenance. In agreement with this, the glucose transporter gene Slc2al that shuttles 
glucose from the blastocoel cavity to ICM cells is downregulated in cultured embryos compared to in vivo embryos (Leppens-Luisier et al., 2001; Uechi et al., 1997). Thus, the decreased availability of glucose may lead to inadequate ATP generation, which could negatively affect the regulation of genomic imprinting as ATP powers a number of epigenetic factors such as chromatin remodelers (reviewed in Hargreaves and Crabtree, 2011). However, it remains unclear why every cultured embryo does not exhibit loss-ofimprinting and every imprinted gene is not equally affected.

\subsubsection{Loss-of-imprinting in placenta induced by ART}

The placenta is more susceptible to loss-of-imprinting when the conceptus is conceived with the use of ART (de Waal et al., 2014; de Waal et al., 2015; Mann et al., 2004; Rivera et al., 2008). Currently, a couple of hypotheses have been put forward to explain why epimutations are more frequent in the extra-embryonic lineages when ART procedures are applied. In the first hypothesis, it is thought that extraembryonic cells are in direct contact with the culture media and are more severely affected by in vitro culture (Mann et al., 2004). However, this hypothesis does not explain why yolk sac, which is derived from primitive endoderm that resides in the inner space of the blastocyst, also exhibits similar severity of loss-of-imprinting (Rivera et al., 2008). Further, administration of hyperstimulation hormones without embryo culture and embryo transfer can induce more severe loss-of-imprinting in placenta compared to the embryo proper (Fortier et al., 2008). Collectively, this evidence suggests that the direct contact of trophectoderm to the culture medium is not the main reason for the differential frequency of epimutation between the embryo and the placenta. Another hypothesis is based on the fact that genomic imprinting is differentially regulated in embryo and placenta. For 
instance, repression of the maternal flanking genes by the long ncRNA Kcnqlotl at the Kcnq1 locus depends on DNA methylation in the embryo but relies on histone modifications in the placenta (Lewis et al., 2004; Umlauf et al., 2004). It is possible that histone modifications are more dynamic and reversible compared to DNA methylation. Thus, the imprinted genes in placenta are more prone to epigenetic errors.

In addition to loss-of-imprinting, ART can also induce aberrant gene expression and DNA methylation at non-imprinted loci in placenta (de Waal et al., 2015). For example, the use of IVF results in decreased transcript abundance of glucose transporter Glut3, which is correlated with the increased DNA methylation level at a $\mathrm{CpG}$ island that is $1000 \mathrm{bp}$ upstream of the transcription start site (de Waal et al., 2015). Currently, the connections between loss-of-imprinting and misregulation of non-imprinted genes remain unclear.

\subsection{Molecular etiology of LOS}

\subsubsection{Loss-of-imprinting in LOS}

LOS was first found to be associated with the epigenetic change in $I G F 2 R$ in sheep (Young et al., 2001). This finding generated questions about the epigenetic likeliness of LOS and BWS as Igf2r is not often imprinted in human (Monk et al., 2006). Later, we observed biallelic expression of the long ncRNA KCNQ1OT1 and loss of methylation of KvDMR1 on the maternal allele in bovine LOS fetuses (Chen et al., 2013), which epimutations account over 50\% of BWS individuals (reviewed in Choufani et al., 2010). More recently, we demonstrated that bovine LOS is a multi-locus loss-of- 
imprinting syndrome (Chen et al., 2015), which is in parallel with the fact that a subset of BWS individuals with hypomethylation at the $K C N Q 1$ locus also exhibits imprinting errors at other loci (Azzi et al., 2009; Bliek et al., 2009; Eggermann et al., 2016). Of particular interest, the number of imprinted genes showing biallelic gene expression is correlated with the bodyweight of LOS fetuses, suggesting that severity of loss-ofimprinting could be an indicator for LOS overgrowth phenotype (Chen et al., 2015). Although some imprinted genes showing loss-of-imprinting in LOS fetuses are known fetal growth regulators such as $I G F 2 R$ and $C D K N 1 C$ (Barlow et al., 1991; Tunster et al., 2011), how misregulation of other imprinted genes contributes to variable phenotypes of LOS is still not understood. In future studies, detailed examination morphology, histology, and metabolic status of LOS fetuses will help establish the relationship between individual imprinted gene/domain and specific phenotypes of LOS.

It should be noted that biallelic expression of imprinted genes in LOS fetuses are not always associated with aberrant DNA methylation at the adjacent DMRs (Chen et al., 2015), which is consistent with the observations reported by several other groups (de Waal et al., 2014; de Waal et al., 2012a; Susiarjo et al., 2013). One possibility is that only specific cell types in a particular tissue express the imprinted gene and are responsible for the monoallelic or biallelic expression pattern detected in the tissue. Thus, abnormal DNA methylation in the specific cell types could be masked by the normal DNA methylation profiles of other cell types that do not express the imprinted gene. Alternatively, aberrant expression of imprinted genes can be due to either misregulation of other epigenetic modifications besides DNA methylation or misregulation of DNA methylation at other DMRs. Another interesting observation is 
that biallelic expression of imprinted genes does not always lead to increased transcript abundance. For example, $I G F 2 R$ is biallelically expressed in kidney, skeletal muscle and liver in all LOS fetuses but exhibits decreased transcript abundance in these tissues compared to controls (Chen et al., 2015). This observation is consistent with previous reports that misregulation of imprinted genes can be independent of allele-specific gene expression or allele-specific DNA methylation (Boucher et al., 2014; Dalgaard et al., 2016). It is likely that additional layer of regulatory factors exist to ensure the fineturning expression of imprinted genes.

It is unclear why the BWS and LOS individuals exhibit variable loss-ofimprinting and variable phenotype. Genetic variation between individuals may not completely explain the variability as the LOS fetuses in our studies shared the same sire and the dams are from the same population of B. t. taurus animals (Chen et al., 2015; Chen et al., 2013). Instead, stochastic epimutation originating from the in vitro oocyte maturation and embryo culture are more plausible causes for the variable loss-ofimprinting observed in LOS fetuses. To understand the mechanisms of imprinting errors induced by these ART procedures, it would be necessary to determine how early these epimutations can be detected in in vitro-derived bovine embryos. As not all embryos exhibit loss-of-imprinting following the use of ART procedures, individual embryos should be used for assessment of allele-specific expression and allele-specific DNA methylation of imprinted genes. 


\subsubsection{Aberrant gene expression at non-imprinted loci in LOS}

Using high throughput RNA sequencing, we found that LOS fetuses exhibited different numbers of DEGs and that each tissue within LOS fetuses had perturbations of distinct gene pathways. These findings indicate that the molecular lesion of LOS is not limited to loss-of-imprinting and the variable phenotypes observed in LOS could be contributed by misregulation of both imprinted and non-imprinted genes. In agreement with this, we found that multiple pathways involved in myoblast proliferation and fusions into myotubes are misregulated in skeletal muscle of LOS fetuses. As proposed by a previous study in sheep LOS (Maxfield et al., 1998), these altered myogenesis pathways may contribute to the large muscles in the oversized LOS fetuses.

It is unclear how the use of ART procedures leads to aberrant gene expression at the non-imprinted loci. It is possible that the misregulation of non-imprinted genes is either an upstream or downstream effect of the ART-induced loss-of-imprinting. In support of this, it has been shown that imprinted genes regulate fetal development through an imprinted gene network in which many non-imprinted genes are also coregulated (Varrault et al., 2006). However, it is also likely that abnormal expression of non-imprinted genes and loss-of-imprinting are independent events. For example, mouse conceptuses generated with the use of embryo transfer can exhibit placentomegaly and junctional zone overgrowth, but the DNA methylation appears normal at several imprinted genes that are associated with the similar phenotypes (de Waal et al., 2015). In future studies, transcriptome analyses of control and LOS fetuses at various developmental stages will help decipher these possibilities. 


\subsubsection{Lack of association between DMRs and DEGs in LOS}

For the purpose of identifying the mechanistic causes of abnormal gene expression in LOS fetuses, we profiled the genome wide DNA methylation at single base resolution of control and LOS skeletal muscle using whole genome bisulfite sequencing. We found numerous local methylation differences between control and LOS fetuses. Surprisingly, only a small percent of DEGs could be directly associated with the identified DMRs between control and LOS, while altered DNA methylation may not be the direct cause of the large number of DEGs identified in LOS. One potential epigenetic modification that mediates the misregulation of the non-imprinted genes is the repressive histone modification $\mathrm{H} 3 \mathrm{~K} 27 \mathrm{me} 3$. H3K27me3 is mostly enriched at the $\mathrm{CpG}$ island promoters of a number of developmentally important genes that are not regulated by DNA methylation (Boulard et al., 2015). It has been shown that Ezh2, the methyltransferase responsible for $\mathrm{H} 3 \mathrm{~K} 27 \mathrm{me} 3$, regulates muscle gene expression and skeletal muscle differentiation (Caretti et al., 2004). Further, impaired reprogramming of H3K27me3 in early embryos compromises the blastocyst development in bovine (Canovas et al., 2012). Collectively, it is possible that H3K27me3 in bovine acts as an epigenetic memory for the use of ART and mediates the abnormal gene expression in later stages.

\subsubsection{Challenges for studying LOS}

Several challenges impede the complete understanding of the etiology of LOS. The most prominent one is that we are still not able to predict which in vitro cultured embryos will develop LOS. A current strategy is to supplement estrus serum in the 
culture media to increase the frequency of LOS fetuses. However, generation of LOS fetuses using this method is not reproducible. For example, production of in vitro embryos at different occasions may yield different number of LOS fetuses or LOS fetuses at each time may show different abnormalities (reviewed in Young et al., 1998). If the molecular signature of LOS can be defined in early embryos, a pre-implantation screen for LOS prior to embryo transfer will increase the efficiency for production of LOS. In addition, it has been recognized that LOS fetuses exhibit a number of misregulated genes and a wide range of abnormalities. Thus, it is also challenging to establish the connection between the altered gene expression and the observed phenotypes in LOS. In future studies, experiments involving genetic manipulation may be necessary to ascertain the causal relationship between the aberrant gene expression and the phenotypic defects in LOS individuals.

\subsection{Conclusions}

The work in this dissertation demonstrates that bovine LOS is a multi-locus lossof-imprinting syndrome, as in a subset of BWS patients. Further, we show that loss-ofimprinting is not the only molecular lesion for LOS and misregulation of both imprinted and non-imprinted genes may contribute to the abnormal phenotypes observed in LOS. Importantly, most aberrant gene expression in LOS is not directly associated with the altered DNA methylation. In addition to providing insights for the etiology of LOS, we also identify novel imprinted genes in bovine and expand the A-to-I RNA editing sites (from $\sim 20$ to 671 ) in bovine fetal tissues. In future studies, continued investigation of 
LOS at various development stages will lay the foundation to predict the occurrence of LOS and help understand the molecular mechanisms of these congenital overgrowth conditions. 


\section{Bibliography}

Abi Habib, W., Azzi, S., Brioude, F., Steunou, V., Thibaud, N., Das Neves, C., Le Jule, M., Chantot-Bastaraud, S., Keren, B., Lyonnet, S., et al. (2014). Extensive investigation of the IGF2/H19 imprinting control region reveals novel OCT4/SOX2 binding site defects associated

with specific methylation patterns in Beckwith-Wiedemann syndrome. Hum Mol Genet 23, 57635773.

Abmayr, S.M., and Pavlath, G.K. (2012). Myoblast fusion: lessons from flies and mice. Development 139, 641-656.

Abu-Amero, S., Monk, D., Frost, J., Preece, M., Stanier, P., and Moore, G.E. (2008). The genetic aetiology of Silver-Russell syndrome. J Med Genet 45, 193-199.

Akalin, A., Kormaksson, M., Li, S., Garrett-Bakelman, F.E., Figueroa, M.E., Melnick, A., and Mason, C.E. (2012). methylKit: a comprehensive R package for the analysis of genome-wide DNA methylation profiles. Genome biology 13, R87.

Al Adhami, H., Evano, B., Le Digarcher, A., Gueydan, C., Dubois, E., Parrinello, H., Dantec, C., Bouschet, T., Varrault, A., and Journot, L. (2015). A systems-level approach to parental genomic imprinting: the imprinted gene network includes extracellular matrix genes and regulates cell cycle exit and differentiation. Genome Res.

Anders, S., McCarthy, D.J., Chen, Y., Okoniewski, M., Smyth, G.K., Huber, W., and Robinson, M.D. (2013). Count-based differential expression analysis of RNA sequencing data using R and Bioconductor. Nature protocols 8, 1765-1786.

Arand, J., Spieler, D., Karius, T., Branco, M.R., Meilinger, D., Meissner, A., Jenuwein, T., Xu, G., Leonhardt, H., Wolf, V., et al. (2012). In vivo control of CpG and non-CpG DNA methylation by DNA methyltransferases. PLoS Genet 8 , e1002750.

Aravin, A.A., Sachidanandam, R., Bourc'his, D., Schaefer, C., Pezic, D., Toth, K.F., Bestor, T., and Hannon, G.J. (2008). A piRNA pathway primed by individual transposons is linked to de novo DNA methylation in mice. Mol Cell 31, 785-799.

Arima, T., Kamikihara, T., Hayashida, T., Kato, K., Inoue, T., Shirayoshi, Y., Oshimura, M., Soejima, H., Mukai, T., and Wake, N. (2005). ZAC, LIT1 (KCNQ1OT1) and p57KIP2 (CDKN1C) are in an imprinted gene network that may play a role in Beckwith-Wiedemann syndrome. Nucleic acids research 33, 2650-2660.

Aronesty, E. (2011). ea-utils : "Command-line tools for processing biological sequencing data"; http://code.google.com/p/ea-utils. 
Azzi, S., Abi Habib, W., and Netchine, I. (2014). Beckwith-Wiedemann and Russell-Silver Syndromes: from new molecular insights to the comprehension of imprinting regulation. Curr Opin Endocrinol Diabetes Obes 21, 30-38.

Azzi, S., Rossignol, S., Steunou, V., Sas, T., Thibaud, N., Danton, F., Le Jule, M., Heinrichs, C., Cabrol, S., Gicquel, C., et al. (2009). Multilocus methylation analysis in a large cohort of 11p15related foetal growth disorders (Russell Silver and Beckwith Wiedemann syndromes) reveals simultaneous loss of methylation at paternal and maternal imprinted loci. Human molecular genetics $18,4724-4733$.

Babak, T., Deveale, B., Armour, C., Raymond, C., Cleary, M.A., van der Kooy, D., Johnson, J.M., and Lim, L.P. (2008). Global survey of genomic imprinting by transcriptome sequencing. Current biology : CB 18, 1735-1741.

Baran, Y., Subramaniam, M., Biton, A., Tukiainen, T., Tsang, E.K., Rivas, M.A., Pirinen, M., Gutierrez-Arcelus, M., Smith, K.S., Kukurba, K.R., et al. (2015). The landscape of genomic imprinting across diverse adult human tissues. Genome research 25, 927-936.

Barau, J., Teissandier, A., Zamudio, N., Roy, S., Nalesso, V., Herault, Y., Guillou, F., and Bourc'his, D. (2016). The DNA methyltransferase DNMT3C protects male germ cells from transposon activity. Science 354, 909-912.

Barlow, D.P., Stoger, R., Herrmann, B.G., Saito, K., and Schweifer, N. (1991). The mouse insulin-like growth factor type-2 receptor is imprinted and closely linked to the Tme locus. Nature 349, 84-87.

Bartolomei, M.S., and Ferguson-Smith, A.C. (2011). Mammalian genomic imprinting. Cold Spring Harbor perspectives in biology 3.

Bartolomei, M.S., Zemel, S., and Tilghman, S.M. (1991). Parental imprinting of the mouse H19 gene. Nature 351, 153-155.

Barton, S.C., Surani, M.A., and Norris, M.L. (1984). Role of paternal and maternal genomes in mouse development. Nature 311,374-376.

Bebbere, D., Bauersachs, S., Furst, R.W., Reichenbach, H.D., Reichenbach, M., Medugorac, I., Ulbrich, S.E., Wolf, E., Ledda, S., and Hiendleder, S. (2013). Tissue-specific and minor interindividual variation in imprinting of IGF2R is a common feature of Bos taurus Concepti and not correlated with fetal weight. PloS one 8 , e59564.

Beckwith, J. (1969). Macroglossia, omphalocele, adrenal cytomegaly, gigantism and hyperplastic visceromegaly. Birth Defects 5, 188-196.

Behboodi, E., Anderson, G.B., BonDurant, R.H., Cargill, S.L., Kreuscher, B.R., Medrano, J.F., and Murray, J.D. (1995). Birth of large calves that developed from in vitro-derived bovine embryos. Theriogenology 44, 227-232. 
Bell, A.C., and Felsenfeld, G. (2000). Methylation of a CTCF-dependent boundary controls imprinted expression of the Igf2 gene. Nature 405, 482-485.

Bertolini, M., Mason, J.B., Beam, S.W., Carneiro, G.F., Sween, M.L., Kominek, D.J., Moyer, A.L., Famula, T.R., Sainz, R.D., and Anderson, G.B. (2002). Morphology and morphometry of in vivo- and in vitro-produced bovine concepti from early pregnancy to term and association with high birth weights. Theriogenology 58, 973-994.

Bervini, S., and Herzog, H. (2013). Mouse models of Prader-Willi Syndrome: a systematic review. Front Neuroendocrinol 34, 107-119.

Beygo, J., Citro, V., Sparago, A., De Crescenzo, A., Cerrato, F., Heitmann, M., Rademacher, K., Guala, A., Enklaar, T., Anichini, C., et al. (2013). The molecular function and clinical phenotype of partial deletions of the IGF2/H19 imprinting control region depends on the spatial arrangement of the remaining CTCF-binding sites. Hum Mol Genet 22, 544-557.

Bianchi, D.W., Wilkins-Haug, L.E., Enders, A.C., and Hay, E.D. (1993). Origin of extraembryonic mesoderm in experimental animals: relevance to chorionic mosaicism in humans. Am J Med Genet 46, 542-550.

Billerey, C., Boussaha, M., Esquerre, D., Rebours, E., Djari, A., Meersseman, C., Klopp, C., Gautheret, D., and Rocha, D. (2014). Identification of large intergenic non-coding RNAs in bovine muscle using next-generation transcriptomic sequencing. BMC genomics 15, 499.

Biressi, S., Molinaro, M., and Cossu, G. (2007). Cellular heterogeneity during vertebrate skeletal muscle development. Developmental biology 308, 281-293.

Bischoff, S.R., Tsai, S., Hardison, N., Motsinger-Reif, A.A., Freking, B.A., Nonneman, D., Rohrer, G., and Piedrahita, J.A. (2009). Characterization of conserved and nonconserved imprinted genes in swine. Biology of reproduction 81, 906-920.

Bliek, J., Verde, G., Callaway, J., Maas, S.M., De Crescenzo, A., Sparago, A., Cerrato, F., Russo, S., Ferraiuolo, S., Rinaldi, M.M., et al. (2009). Hypomethylation at multiple maternally methylated imprinted regions including PLAGL1 and GNAS loci in Beckwith-Wiedemann syndrome. European journal of human genetics : EJHG 17, 611-619.

Boucher, J., Charalambous, M., Zarse, K., Mori, M.A., Kleinridders, A., Ristow, M., FergusonSmith, A.C., and Kahn, C.R. (2014). Insulin and insulin-like growth factor 1 receptors are required for normal expression of imprinted genes. Proc Natl Acad Sci U S A 111, 14512-14517.

Boulard, M., Edwards, J.R., and Bestor, T.H. (2015). FBXL10 protects Polycomb-bound genes from hypermethylation. Nat Genet 47, 479-485.

Bourc'his, D., and Bestor, T.H. (2004). Meiotic catastrophe and retrotransposon reactivation in male germ cells lacking Dnmt3L. Nature 431, 96-99. 
Bourc'his, D., Xu, G.L., Lin, C.S., Bollman, B., and Bestor, T.H. (2001). Dnmt3L and the establishment of maternal genomic imprints. Science 294, 2536-2539.

Brannan, C.I., Dees, E.C., Ingram, R.S., and Tilghman, S.M. (1990). The product of the H19 gene may function as an RNA. Mol Cell Biol 10, 28-36.

Buiting, K. (2010). Prader-Willi syndrome and Angelman syndrome. Am J Med Genet C Semin Med Genet 154C, 365-376.

Cai, X., and Cullen, B.R. (2007). The imprinted H19 noncoding RNA is a primary microRNA precursor. RNA 13, 313-316.

Canovas, S., Cibelli, J.B., and Ross, P.J. (2012). Jumonji domain-containing protein 3 regulates histone 3 lysine 27 methylation during bovine preimplantation development. Proc Natl Acad Sci U S A 109, 2400-2405.

Caretti, G., Di Padova, M., Micales, B., Lyons, G.E., and Sartorelli, V. (2004). The Polycomb Ezh2 methyltransferase regulates muscle gene expression and skeletal muscle differentiation. Genes Dev 18, 2627-2638.

Carmell, M.A., Girard, A., van de Kant, H.J., Bourc'his, D., Bestor, T.H., de Rooij, D.G., and Hannon, G.J. (2007). MIWI2 is essential for spermatogenesis and repression of transposons in the mouse male germline. Dev Cell 12, 503-514.

Caspary, T., Cleary, M.A., Perlman, E.J., Zhang, P., Elledge, S.J., and Tilghman, S.M. (1999). Oppositely imprinted genes p57(Kip2) and igf2 interact in a mouse model for BeckwithWiedemann syndrome. Genes Dev 13, 3115-3124.

CDC (2013). 2011 Assisted Reproductive Technology National Summary Report. Centers for Disease Control and Prevention, American Society for Reproductive Medicine, Society for Assisted Reproductive Technology.

Champagne, F.A., Curley, J.P., Swaney, W.T., Hasen, N.S., and Keverne, E.B. (2009). Paternal influence on female behavior: the role of Peg3 in exploration, olfaction, and neuroendocrine regulation of maternal behavior of female mice. Behav Neurosci 123, 469-480.

Chen, Z., Hagen, D.E., Elsik, C.G., Ji, T., Morris, C.J., Moon, L.E., and Rivera, R.M. (2015). Characterization of global loss of imprinting in fetal overgrowth syndrome induced by assisted reproduction. Proceedings of the National Academy of Sciences of the United States of America $112,4618-4623$.

Chen, Z., Hagen, D.E., Wang, J., Elsik, C.G., Ji, T., Siqueira, L.G., Hansen, P.J., and Rivera, R.M. (2016). Global assessment of imprinted gene expression in the bovine conceptus by next generation sequencing. Epigenetics : official journal of the DNA Methylation Society 11, 501 516. 
Chen, Z., Robbins, K.M., Wells, K.D., and Rivera, R.M. (2013). Large offspring syndrome: a bovine model for the human loss-of-imprinting overgrowth syndrome Beckwith-Wiedemann. Epigenetics : official journal of the DNA Methylation Society 8, 591-601.

Chiesa, N., De Crescenzo, A., Mishra, K., Perone, L., Carella, M., Palumbo, O., Mussa, A., Sparago, A., Cerrato, F., Russo, S., et al. (2012). The KCNQ1OT1 imprinting control region and non-coding RNA: new properties derived from the study of Beckwith-Wiedemann syndrome and Silver-Russell syndrome cases. Hum Mol Genet 21, 10-25.

Choo, J.H., Kim, J.D., and Kim, J. (2008). Imprinting of an evolutionarily conserved antisense transcript gene APeg3. Gene 409, 28-33.

Chotalia, M., Smallwood, S.A., Ruf, N., Dawson, C., Lucifero, D., Frontera, M., James, K., Dean, W., and Kelsey, G. (2009). Transcription is required for establishment of germline methylation marks at imprinted genes. Genes Dev 23, 105-117.

Choufani, S., Shuman, C., and Weksberg, R. (2010). Beckwith-Wiedemann syndrome. American journal of medical genetics Part C, Seminars in medical genetics 154C, 343-354.

Choufani, S., Shuman, C., and Weksberg, R. (2013). Molecular findings in Beckwith-Wiedemann syndrome. Am J Med Genet C Semin Med Genet 163C, 131-140.

Ciccone, D.N., Su, H., Hevi, S., Gay, F., Lei, H., Bajko, J., Xu, G., Li, E., and Chen, T. (2009). $\mathrm{KDM} 1 \mathrm{~B}$ is a histone $\mathrm{H} 3 \mathrm{~K} 4$ demethylase required to establish maternal genomic imprints. Nature 461, 415-418.

Clayton-Smith, J., and Laan, L. (2003). Angelman syndrome: a review of the clinical and genetic aspects. J Med Genet 40, 87-95.

Cleaton, M.A., Dent, C.L., Howard, M., Corish, J.A., Gutteridge, I., Sovio, U., Gaccioli, F., Takahashi, N., Bauer, S.R., Charnock-Jones, D.S., et al. (2016). Fetus-derived DLK1 is required for maternal metabolic adaptations to pregnancy and is associated with fetal growth restriction. Nat Genet 48, 1473-1480.

Constancia, M., Hemberger, M., Hughes, J., Dean, W., Ferguson-Smith, A., Fundele, R., Stewart, F., Kelsey, G., Fowden, A., Sibley, C., et al. (2002). Placental-specific IGF-II is a major modulator of placental and fetal growth. Nature 417, 945-948.

Cortellino, S., Xu, J., Sannai, M., Moore, R., Caretti, E., Cigliano, A., Le Coz, M., Devarajan, K., Wessels, A., Soprano, D., et al. (2011). Thymine DNA glycosylase is essential for active DNA demethylation by linked deamination-base excision repair. Cell 146, 67-79.

Court, F., Martin-Trujillo, A., Romanelli, V., Garin, I., Iglesias-Platas, I., Salafsky, I., Guitart, M., Perez de Nanclares, G., Lapunzina, P., and Monk, D. (2013). Genome-wide allelic methylation analysis reveals disease-specific susceptibility to multiple methylation defects in imprinting syndromes. Human mutation 34, 595-602. 
Cox, M.P., Peterson, D.A., and Biggs, P.J. (2010). SolexaQA: At-a-glance quality assessment of Illumina second-generation sequencing data. BMC bioinformatics 11, 485.

Dahl, J.A., Jung, I., Aanes, H., Greggains, G.D., Manaf, A., Lerdrup, M., Li, G., Kuan, S., Li, B., Lee, A.Y., et al. (2016). Broad histone H3K4me3 domains in mouse oocytes modulate maternalto-zygotic transition. Nature 537, 548-552.

Dalgaard, K., Landgraf, K., Heyne, S., Lempradl, A., Longinotto, J., Gossens, K., Ruf, M., Orthofer, M., Strogantsev, R., Selvaraj, M., et al. (2016). Trim28 Haploinsufficiency Triggers Bistable Epigenetic Obesity. Cell 164, 353-364.

Danecek, P., Nellaker, C., McIntyre, R.E., Buendia-Buendia, J.E., Bumpstead, S., Ponting, C.P., Flint, J., Durbin, R., Keane, T.M., and Adams, D.J. (2012). High levels of RNA-editing site conservation amongst 15 laboratory mouse strains. Genome biology 13, 26.

Das, P.J., Chowdhary, B.P., and Raudsepp, T. (2009). Characterization of the bovine pseudoautosomal region and comparison with sheep, goat, and other mammalian pseudoautosomal regions. Cytogenetic and genome research 126, 139-147.

Das, P.P., Hendrix, D.A., Apostolou, E., Buchner, A.H., Canver, M.C., Beyaz, S., Ljuboja, D., Kuintzle, R., Kim, W., Karnik, R., et al. (2015). PRC2 Is Required to Maintain Expression of the Maternal Gt12-Rian-Mirg Locus by Preventing De Novo DNA Methylation in Mouse Embryonic Stem Cells. Cell Rep 12, 1456-1470.

Davis, T.L., Trasler, J.M., Moss, S.B., Yang, G.J., and Bartolomei, M.S. (1999). Acquisition of the H19 methylation imprint occurs differentially on the parental alleles during spermatogenesis. Genomics 58, 18-28.

Davis, T.L., Yang, G.J., McCarrey, J.R., and Bartolomei, M.S. (2000). The H19 methylation imprint is erased and re-established differentially on the parental alleles during male germ cell development. Hum Mol Genet 9, 2885-2894.

Dawlaty, M.M., Breiling, A., Le, T., Raddatz, G., Barrasa, M.I., Cheng, A.W., Gao, Q., Powell, B.E., Li, Z., Xu, M., et al. (2013). Combined deficiency of Tet1 and Tet2 causes epigenetic abnormalities but is compatible with postnatal development. Dev Cell 24, 310-323.

de Waal, E., Mak, W., Calhoun, S., Stein, P., Ord, T., Krapp, C., Coutifaris, C., Schultz, R.M., and Bartolomei, M.S. (2014). In vitro culture increases the frequency of stochastic epigenetic errors at imprinted genes in placental tissues from mouse concepti produced through assisted reproductive technologies. Biol Reprod 90, 22.

de Waal, E., Vrooman, L.A., Fischer, E., Ord, T., Mainigi, M.A., Coutifaris, C., Schultz, R.M., and Bartolomei, M.S. (2015). The cumulative effect of assisted reproduction procedures on placental development and epigenetic perturbations in a mouse model. Hum Mol Genet 24, 69756985 . 
de Waal, E., Yamazaki, Y., Ingale, P., Bartolomei, M., Yanagimachi, R., and McCarrey, J.R. (2012a). Primary epimutations introduced during intracytoplasmic sperm injection (ICSI) are corrected by germline-specific epigenetic reprogramming. Proc Natl Acad Sci U S A 109, 41634168 .

de Waal, E., Yamazaki, Y., Ingale, P., Bartolomei, M.S., Yanagimachi, R., and McCarrey, J.R. (2012b). Gonadotropin stimulation contributes to an increased incidence of epimutations in ICSIderived mice. Hum Mol Genet 21, 4460-4472.

DeBaun, M.R., Niemitz, E.L., and Feinberg, A.P. (2003). Association of in vitro fertilization with Beckwith-Wiedemann syndrome and epigenetic alterations of LIT1 and H19. American journal of human genetics $72,156-160$.

DeChiara, T.M., Robertson, E.J., and Efstratiadis, A. (1991). Parental imprinting of the mouse insulin-like growth factor II gene. Cell 64, 849-859.

Degner, J.F., Marioni, J.C., Pai, A.A., Pickrell, J.K., Nkadori, E., Gilad, Y., and Pritchard, J.K. (2009). Effect of read-mapping biases on detecting allele-specific expression from RNAsequencing data. Bioinformatics 25, 3207-3212.

Deng, Q., Ramskold, D., Reinius, B., and Sandberg, R. (2014). Single-cell RNA-seq reveals dynamic, random monoallelic gene expression in mammalian cells. Science 343, 193-196.

Denomme, M.M., and Mann, M.R. (2012). Genomic imprints as a model for the analysis of epigenetic stability during assisted reproductive technologies. Reproduction (Cambridge, England) $144,393-409$.

Denomme, M.M., Zhang, L., and Mann, M.R. (2011). Embryonic imprinting perturbations do not originate from superovulation-induced defects in DNA methylation acquisition. Fertil Steril 96, 734-738 e732.

DePristo, M.A., Banks, E., Poplin, R., Garimella, K.V., Maguire, J.R., Hartl, C., Philippakis, A.A., del Angel, G., Rivas, M.A., Hanna, M., et al. (2011). A framework for variation discovery and genotyping using next-generation DNA sequencing data. Nature genetics 43, 491-498.

DeVeale, B., van der Kooy, D., and Babak, T. (2012). Critical evaluation of imprinted gene expression by RNA-Seq: a new perspective. PLoS genetics 8 , e1002600.

Dhayalan, A., Rajavelu, A., Rathert, P., Tamas, R., Jurkowska, R.Z., Ragozin, S., and Jeltsch, A. (2010). The Dnmt3a PWWP domain reads histone 3 lysine 36 trimethylation and guides DNA methylation. J Biol Chem 285, 26114-26120.

Diaz-Meyer, N., Day, C.D., Khatod, K., Maher, E.R., Cooper, W., Reik, W., Junien, C., Graham, G., Algar, E., Der Kaloustian, V.M., et al. (2003). Silencing of CDKN1C (p57KIP2) is associated with hypomethylation at KvDMR1 in Beckwith-Wiedemann syndrome. J Med Genet 40, 797801 . 
Dindot, S.V., Kent, K.C., Evers, B., Loskutoff, N., Womack, J., and Piedrahita, J.A. (2004). Conservation of genomic imprinting at the XIST, IGF2, and GTL2 loci in the bovine. Mamm Genome 15, 966-974.

Ding, F., Li, H.H., Zhang, S., Solomon, N.M., Camper, S.A., Cohen, P., and Francke, U. (2008). SnoRNA Snord1 16 (Pwcr1/MBII-85) deletion causes growth deficiency and hyperphagia in mice. PLoS One 3, e1709.

Dobbs, K.B., Gagne, D., Fournier, E., Dufort, I., Robert, C., Block, J., Sirard, M.A., Bonilla, L., Ealy, A.D., Loureiro, B., et al. (2014). Sexual dimorphism in developmental programming of the bovine preimplantation embryo caused by colony-stimulating factor 2 . Biology of reproduction 91,80 .

Edwards, C.A., and Ferguson-Smith, A.C. (2007). Mechanisms regulating imprinted genes in clusters. Curr Opin Cell Biol 19, 281-289.

Eggenschwiler, J., Ludwig, T., Fisher, P., Leighton, P.A., Tilghman, S.M., and Efstratiadis, A. (1997). Mouse mutant embryos overexpressing IGF-II exhibit phenotypic features of the Beckwith-Wiedemann and Simpson-Golabi-Behmel syndromes. Genes \& development 11, 31283142 .

Eggermann, T., Algar, E., Lapunzina, P., Mackay, D., Maher, E.R., Mannens, M., Netchine, I., Prawitt, D., Riccio, A., Temple, I.K., et al. (2014). Clinical utility gene card for: BeckwithWiedemann Syndrome. European journal of human genetics : EJHG 22.

Eggermann, T., Brioude, F., Russo, S., Lombardi, M.P., Bliek, J., Maher, E.R., Larizza, L., Prawitt, D., Netchine, I., Gonzales, M., et al. (2016). Prenatal molecular testing for BeckwithWiedemann and Silver-Russell syndromes: a challenge for molecular analysis and genetic counseling. European journal of human genetics : EJHG 24, 784-793.

Elsik, C.G., Unni, D.R., Diesh, C.M., Tayal, A., Emery, M.L., Nguyen, H.N., and Hagen, D.E. (2015). Bovine Genome Database: new tools for gleaning function from the Bos taurus genome. Nucleic acids research.

European, I.V.F.M.C., European Society of Human, R., Embryology, Kupka, M.S., D'Hooghe, T., Ferraretti, A.P., de Mouzon, J., Erb, K., Castilla, J.A., Calhaz-Jorge, C., et al. (2016). Assisted reproductive technology in Europe, 2011: results generated from European registers by ESHRE. Human reproduction 31, 233-248.

Evans, H.K., Wylie, A.A., Murphy, S.K., and Jirtle, R.L. (2001). The neuronatin gene resides in a "micro-imprinted" domain on human chromosome 20q11.2. Genomics 77, 99-104.

Farin, P.W., and Farin, C.E. (1995). Transfer of bovine embryos produced in vivo or in vitro: survival and fetal development. Biol Reprod 52, 676-682. 
Farin, P.W., Piedrahita, J.A., and Farin, C.E. (2006). Errors in development of fetuses and placentas from in vitro-produced bovine embryos. Theriogenology 65, 178-191.

Ferguson, E.M., and Leese, H.J. (1999). Triglyceride content of bovine oocytes and early embryos. Journal of Reproduction and Fertility 116, 373-378.

Fitzpatrick, G.V., Soloway, P.D., and Higgins, M.J. (2002). Regional loss of imprinting and growth deficiency in mice with a targeted deletion of KvDMR1. Nat Genet 32, 426-431.

Fortier, A.L., Lopes, F.L., Darricarrere, N., Martel, J., and Trasler, J.M. (2008). Superovulation alters the expression of imprinted genes in the midgestation mouse placenta. Hum Mol Genet 17, $1653-1665$.

Frank, D., Fortino, W., Clark, L., Musalo, R., Wang, W., Saxena, A., Li, C.M., Reik, W., Ludwig, T., and Tycko, B. (2002). Placental overgrowth in mice lacking the imprinted gene Ipl. Proc Natl Acad Sci U S A 99, 7490-7495.

Gabory, A., Ripoche, M.A., Le Digarcher, A., Watrin, F., Ziyyat, A., Forne, T., Jammes, H., Ainscough, J.F., Surani, M.A., Journot, L., et al. (2009). H19 acts as a trans regulator of the imprinted gene network controlling growth in mice. Development 136, 3413-3421.

Gardiner-Garden, M., and Frommer, M. (1987). CpG islands in vertebrate genomes. J Mol Biol 196, 261-282.

Garfield, A.S., Cowley, M., Smith, F.M., Moorwood, K., Stewart-Cox, J.E., Gilroy, K., Baker, S., Xia, J., Dalley, J.W., Hurst, L.D., et al. (2011). Distinct physiological and behavioural functions for parental alleles of imprinted Grb10. Nature 469, 534-538.

Garry, D.J., Bassel-Duby, R.S., Richardson, J.A., Grayson, J., Neufer, P.D., and Williams, R.S. (1996). Postnatal development and plasticity of specialized muscle fiber characteristics in the hindlimb. Dev Genet 19, 146-156.

Gentile, J.K., Tan, W.H., Horowitz, L.T., Bacino, C.A., Skinner, S.A., Barbieri-Welge, R., BauerCarlin, A., Beaudet, A.L., Bichell, T.J., Lee, H.S., et al. (2010). A neurodevelopmental survey of Angelman syndrome with genotype-phenotype correlations. J Dev Behav Pediatr 31, 592-601.

Gicquel, C., Gaston, V., Mandelbaum, J., Siffroi, J.P., Flahault, A., and Le Bouc, Y. (2003). In vitro fertilization may increase the risk of Beckwith-Wiedemann syndrome related to the abnormal imprinting of the KCN1OT gene. American journal of human genetics 72, 1338-1341.

Ginsburg, M., Snow, M.H., and McLaren, A. (1990). Primordial germ cells in the mouse embryo during gastrulation. Development 110, 521-528.

Grafodatskaya, D., Cytrynbaum, C., and Weksberg, R. (2013). The health risks of ART. EMBO reports $14,129-135$. 
Gu, T.P., Guo, F., Yang, H., Wu, H.P., Xu, G.F., Liu, W., Xie, Z.G., Shi, L., He, X., Jin, S.G., et al. (2011). The role of Tet3 DNA dioxygenase in epigenetic reprogramming by oocytes. Nature 477, 606-610.

Guibert, S., Forne, T., and Weber, M. (2012). Global profiling of DNA methylation erasure in mouse primordial germ cells. Genome Res 22, 633-641.

Guillemot, F., Nagy, A., Auerbach, A., Rossant, J., and Joyner, A.L. (1994). Essential role of Mash-2 in extraembryonic development. Nature 371, 333-336.

Guo, X., Wang, L., Li, J., Ding, Z., Xiao, J., Yin, X., He, S., Shi, P., Dong, L., Li, G., et al. (2015). Structural insight into autoinhibition and histone H3-induced activation of DNMT3A. Nature 517, 640-644.

Hackett, J.A., Sengupta, R., Zylicz, J.J., Murakami, K., Lee, C., Down, T.A., and Surani, M.A. (2013). Germline DNA demethylation dynamics and imprint erasure through 5hydroxymethylcytosine. Science 339, 448-452.

Hajkova, P., Erhardt, S., Lane, N., Haaf, T., El-Maarri, O., Reik, W., Walter, J., and Surani, M.A. (2002). Epigenetic reprogramming in mouse primordial germ cells. Mech Dev 117, 15-23.

Halliday, J., Oke, K., Breheny, S., Algar, E., and D, J.A. (2004). Beckwith-Wiedemann syndrome and IVF: a case-control study. American journal of human genetics 75, 526-528.

Hanna, C.W., and Kelsey, G. (2014). The specification of imprints in mammals. Heredity (Edinb) $113,176-183$.

Hansen, K.D., Langmead, B., and Irizarry, R.A. (2012). BSmooth: from whole genome bisulfite sequencing reads to differentially methylated regions. Genome biology $13, \mathrm{R} 83$.

Hao, Y., Crenshaw, T., Moulton, T., Newcomb, E., and Tycko, B. (1993). Tumour-suppressor activity of H19 RNA. Nature 365, 764-767.

Hargreaves, D.C., and Crabtree, G.R. (2011). ATP-dependent chromatin remodeling: genetics, genomics and mechanisms. Cell Res 21,396-420.

Hark, A.T., Schoenherr, C.J., Katz, D.J., Ingram, R.S., Levorse, J.M., and Tilghman, S.M. (2000). CTCF mediates methylation-sensitive enhancer-blocking activity at the H19/Igf2 locus. Nature 405, 486-489.

He, Y., and Ecker, J.R. (2015). Non-CG Methylation in the Human Genome. Annu Rev Genomics Hum Genet 16, 55-77. 
He, Y.F., Li, B.Z., Li, Z., Liu, P., Wang, Y., Tang, Q., Ding, J., Jia, Y., Chen, Z., Li, L., et al. (2011). Tet-mediated formation of 5-carboxylcytosine and its excision by TDG in mammalian DNA. Science 333, 1303-1307.

Higuchi, M., Maas, S., Single, F.N., Hartner, J., Rozov, A., Burnashev, N., Feldmeyer, D., Sprengel, R., and Seeburg, P.H. (2000). Point mutation in an AMPA receptor gene rescues lethality in mice deficient in the RNA-editing enzyme ADAR2. Nature 406, 78-81.

Hill, J.R. (2014). Incidence of abnormal offspring from cloning and other assisted reproductive technologies. Annu Rev Anim Biosci 2, 307-321.

Hirasawa, R., Chiba, H., Kaneda, M., Tajima, S., Li, E., Jaenisch, R., and Sasaki, H. (2008). Maternal and zygotic Dnmt1 are necessary and sufficient for the maintenance of DNA methylation imprints during preimplantation development. Genes Dev 22, 1607-1616.

Hori, N., Nagai, M., Hirayama, M., Hirai, T., Matsuda, K., Hayashi, M., Tanaka, T., Ozawa, T., and Horike, S. (2010). Aberrant CpG methylation of the imprinting control region KvDMR1 detected in assisted reproductive technology-produced calves and pathogenesis of large offspring syndrome. Animal reproduction science 122, 303-312.

Howell, C.Y., Bestor, T.H., Ding, F., Latham, K.E., Mertineit, C., Trasler, J.M., and Chaillet, J.R. (2001). Genomic imprinting disrupted by a maternal effect mutation in the Dnmt1 gene. Cell 104, 829-838.

Huang da, W., Sherman, B.T., and Lempicki, R.A. (2009). Systematic and integrative analysis of large gene lists using DAVID bioinformatics resources. Nature protocols 4, 44-57.

Huang, D.W., Sherman, B.T., Tan, Q., Kir, J., Liu, D., Bryant, D., Guo, Y., Stephens, R., Baseler, M.W., Lane, H.C., et al. (2007). DAVID Bioinformatics Resources: expanded annotation database and novel algorithms to better extract biology from large gene lists. Nucleic acids research 35, W169-175.

Hubertus, J., Zitzmann, F., Trippel, F., Muller-Hocker, J., Stehr, M., von Schweinitz, D., and Kappler, R. (2013). Selective methylation of CpGs at regulatory binding sites controls NNAT expression in Wilms tumors. PloS one 8, e67605.

Hur, S.K., Freschi, A., Ideraabdullah, F., Thorvaldsen, J.L., Luense, L.J., Weller, A.H., Berger, S.L., Cerrato, F., Riccio, A., and Bartolomei, M.S. (2016). Humanized H19/Igf2 locus reveals diverged imprinting mechanism between mouse and human and reflects Silver-Russell syndrome phenotypes. Proc Natl Acad Sci U S A 113, 10938-10943.

Huynh, K.D., and Lee, J.T. (2001). Imprinted X inactivation in eutherians: a model of gametic execution and zygotic relaxation. Curr Opin Cell Biol 13, 690-697.

IETS (2013). 2012 Statistics of embryo collection and transfer in domestic farm animals. International Embryo Transfer Society. 
Ingleby, F.C., Flis, I., and Morrow, E.H. (2014a). Sex-Biased Gene Expression and Sexual Conflict throughout Development. Cold Spring Harbor perspectives in biology 6.

Ingleby, F.C., Flis, I., and Morrow, E.H. (2014b). Sex-biased gene expression and sexual conflict throughout development. Cold Spring Harb Perspect Biol 7, a017632.

Inoue, A., Shen, L., Dai, Q., He, C., and Zhang, Y. (2011). Generation and replication-dependent dilution of $5 \mathrm{fC}$ and $5 \mathrm{caC}$ during mouse preimplantation development. Cell Res 21, 1670-1676.

Inoue, A., Shen, L., Matoba, S., and Zhang, Y. (2015). Haploinsufficiency, but not defective paternal $5 \mathrm{mC}$ oxidation, accounts for the developmental defects of maternal Tet3 knockouts. Cell Rep 10, 463-470.

Inoue, A., and Zhang, Y. (2011). Replication-dependent loss of 5-hydroxymethylcytosine in mouse preimplantation embryos. Science 334, 194.

Ito, S., D'Alessio, A.C., Taranova, O.V., Hong, K., Sowers, L.C., and Zhang, Y. (2010). Role of Tet proteins in $5 \mathrm{mC}$ to $5 \mathrm{hmC}$ conversion, ES-cell self-renewal and inner cell mass specification. Nature 466, 1129-1133.

Ito, S., Shen, L., Dai, Q., Wu, S.C., Collins, L.B., Swenberg, J.A., He, C., and Zhang, Y. (2011). Tet proteins can convert 5-methylcytosine to 5-formylcytosine and 5-carboxylcytosine. Science $333,1300-1303$.

Itou, D., Shiromoto, Y., Yukiho, S.Y., Ishii, C., Nishimura, T., Ogonuki, N., Ogura, A., Hasuwa, H., Fujihara, Y., Kuramochi-Miyagawa, S., et al. (2015). Induction of DNA methylation by artificial piRNA production in male germ cells. Curr Biol 25, 901-906.

Jiang, Y.H., Armstrong, D., Albrecht, U., Atkins, C.M., Noebels, J.L., Eichele, G., Sweatt, J.D., and Beaudet, A.L. (1998). Mutation of the Angelman ubiquitin ligase in mice causes increased cytoplasmic p53 and deficits of contextual learning and long-term potentiation. Neuron 21, 799811.

Kagitani, F., Kuroiwa, Y., Wakana, S., Shiroishi, T., Miyoshi, N., Kobayashi, S., Nishida, M., Kohda, T., Kaneko-Ishino, T., and Ishino, F. (1997). Peg5/Neuronatin is an imprinted gene located on sub-distal chromosome 2 in the mouse. Nucleic acids research 25, 3428-3432.

Kagiwada, S., Kurimoto, K., Hirota, T., Yamaji, M., and Saitou, M. (2013). Replication-coupled passive DNA demethylation for the erasure of genome imprints in mice. EMBO J 32, 340-353.

Kalish, J.M., Jiang, C., and Bartolomei, M.S. (2014). Epigenetics and imprinting in human disease. Int J Dev Biol 58, 291-298.

Kaneda, M., Okano, M., Hata, K., Sado, T., Tsujimoto, N., Li, E., and Sasaki, H. (2004). Essential role for de novo DNA methyltransferase Dnmt3a in paternal and maternal imprinting. Nature 429, 900-903. 
Kannenberg, K., Urban, C., and Binder, G. (2012). Increased incidence of aberrant DNA methylation within diverse imprinted gene loci outside of IGF2/H19 in Silver-Russell syndrome. Clin Genet 81, 366-377.

Kato, Y., Kaneda, M., Hata, K., Kumaki, K., Hisano, M., Kohara, Y., Okano, M., Li, E., Nozaki, M., and Sasaki, H. (2007). Role of the Dnmt3 family in de novo methylation of imprinted and repetitive sequences during male germ cell development in the mouse. Hum Mol Genet 16, 22722280.

Keniry, A., Oxley, D., Monnier, P., Kyba, M., Dandolo, L., Smits, G., and Reik, W. (2012). The H19 lincRNA is a developmental reservoir of miR-675 that suppresses growth and Igf1r. Nat Cell Biol 14, 659-665.

Kent, W.J. (2002). BLAT--the BLAST-like alignment tool. Genome research 12, 656-664.

Kim, D., Langmead, B., and Salzberg, S.L. (2015). HISAT: a fast spliced aligner with low memory requirements. Nature methods 12, 357-360.

Kim, D., Pertea, G., Trapnell, C., Pimentel, H., Kelley, R., and Salzberg, S.L. (2013). TopHat2: accurate alignment of transcriptomes in the presence of insertions, deletions and gene fusions. Genome biology 14, R36.

Kishino, T., Lalande, M., and Wagstaff, J. (1997). UBE3A/E6-AP mutations cause Angelman syndrome. Nat Genet 15, 70-73.

Kobayashi, H., Sakurai, T., Imai, M., Takahashi, N., Fukuda, A., Yayoi, O., Sato, S., Nakabayashi, K., Hata, K., Sotomaru, Y., et al. (2012). Contribution of intragenic DNA methylation in mouse gametic DNA methylomes to establish oocyte-specific heritable marks. PLoS Genet 8, e1002440.

Kono, T., Obata, Y., Wu, Q., Niwa, K., Ono, Y., Yamamoto, Y., Park, E.S., Seo, J.S., and Ogawa, H. (2004). Birth of parthenogenetic mice that can develop to adulthood. Nature 428, 860864.

Koufos, A., Grundy, P., Morgan, K., Aleck, K.A., Hadro, T., Lampkin, B.C., Kalbakji, A., and Cavenee, W.K. (1989). Familial Wiedemann-Beckwith syndrome and a second Wilms tumor locus both map to 11p15.5. Am J Hum Genet 44, 711-719.

Krueger, F., and Andrews, S.R. (2011). Bismark: a flexible aligner and methylation caller for Bisulfite-Seq applications. Bioinformatics 27, 1571-1572.

Kuentz, P., Bailly, A., Faure, A.C., Blagosklonov, O., Amiot, C., Bresson, J.L., and Roux, C. (2011). Child with Beckwith-Wiedemann syndrome born after assisted reproductive techniques to an human immunodeficiency virus serodiscordant couple. Fertility and sterility 96, e35-38. 
Kupka, M.S., Ferraretti, A.P., de Mouzon, J., Erb, K., D'Hooghe, T., Castilla, J.A., Calhaz-Jorge, C., De Geyter, C., and Goossens, V. (2014). Assisted reproductive technology in Europe, 2010: results generated from European registers by ESHREdagger. Human reproduction 27.

Kuramochi-Miyagawa, S., Watanabe, T., Gotoh, K., Totoki, Y., Toyoda, A., Ikawa, M., Asada, N., Kojima, K., Yamaguchi, Y., Ijiri, T.W., et al. (2008). DNA methylation of retrotransposon genes is regulated by Piwi family members MILI and MIWI2 in murine fetal testes. Genes Dev $22,908-917$.

Langfelder, P., and Horvath, S. (2008). WGCNA: an R package for weighted correlation network analysis. BMC bioinformatics 9,559 .

Langmead, B., and Salzberg, S.L. (2012). Fast gapped-read alignment with Bowtie 2. Nature methods $9,357-359$.

Langmead, B., Trapnell, C., Pop, M., and Salzberg, S.L. (2009). Ultrafast and memory-efficient alignment of short DNA sequences to the human genome. Genome biology 10, R25.

Latos, P.A., Pauler, F.M., Koerner, M.V., Senergin, H.B., Hudson, Q.J., Stocsits, R.R., Allhoff, W., Stricker, S.H., Klement, R.M., Warczok, K.E., et al. (2012). Airn transcriptional overlap, but not its lncRNA products, induces imprinted Igf2r silencing. Science 338, 1469-1472.

Laurin, M., Fradet, N., Blangy, A., Hall, A., Vuori, K., and Cote, J.F. (2008). The atypical Rac activator Dock180 (Dock1) regulates myoblast fusion in vivo. Proceedings of the National Academy of Sciences of the United States of America 105, 15446-15451.

Lazzari, G., Wrenzycki, C., Herrmann, D., Duchi, R., Kruip, T., Niemann, H., and Galli, C. (2002). Cellular and molecular deviations in bovine in vitro-produced embryos are related to the large offspring syndrome. Biology of reproduction 67, 767-775.

Lee, D.H., Singh, P., Tsai, S.Y., Oates, N., Spalla, A., Spalla, C., Brown, L., Rivas, G., Larson, G., Rauch, T.A., et al. (2010). CTCF-dependent chromatin bias constitutes transient epigenetic memory of the mother at the H19-Igf2 imprinting control region in prospermatogonia. PLoS Genet 6, e1001224.

Lee, J.T., and Bartolomei, M.S. (2013). X-inactivation, imprinting, and long noncoding RNAs in health and disease. Cell 152, 1308-1323.

Lefebvre, L., Viville, S., Barton, S.C., Ishino, F., Keverne, E.B., and Surani, M.A. (1998). Abnormal maternal behaviour and growth retardation associated with loss of the imprinted gene Mest. Nat Genet 20, 163-169.

Leighton, P.A., Ingram, R.S., Eggenschwiler, J., Efstratiadis, A., and Tilghman, S.M. (1995a). Disruption of imprinting caused by deletion of the H19 gene region in mice. Nature 375, 34-39. 
Leighton, P.A., Saam, J.R., Ingram, R.S., Stewart, C.L., and Tilghman, S.M. (1995b). An enhancer deletion affects both H19 and Igf2 expression. Genes Dev 9, 2079-2089.

Leppens-Luisier, G., Urner, F., and Sakkas, D. (2001). Facilitated glucose transporters play a crucial role throughout mouse preimplantation embryo development. Hum Reprod 16, 12291236.

Levin, J.Z., Yassour, M., Adiconis, X., Nusbaum, C., Thompson, D.A., Friedman, N., Gnirke, A., and Regev, A. (2010). Comprehensive comparative analysis of strand-specific RNA sequencing methods. Nature methods 7, 709-715.

Lewis, A., Mitsuya, K., Umlauf, D., Smith, P., Dean, W., Walter, J., Higgins, M., Feil, R., and Reik, W. (2004). Imprinting on distal chromosome 7 in the placenta involves repressive histone methylation independent of DNA methylation. Nat Genet 36, 1291-1295.

Li, H. (2011). A statistical framework for SNP calling, mutation discovery, association mapping and population genetical parameter estimation from sequencing data. Bioinformatics 27, 29872993.

Li, H., and Durbin, R. (2009). Fast and accurate short read alignment with Burrows-Wheeler transform. Bioinformatics 25, 1754-1760.

Li, H., Handsaker, B., Wysoker, A., Fennell, T., Ruan, J., Homer, N., Marth, G., Abecasis, G., Durbin, R., and Genome Project Data Processing, S. (2009). The Sequence Alignment/Map format and SAMtools. Bioinformatics 25, 2078-2079.

Li, J.B., and Church, G.M. (2013). Deciphering the functions and regulation of brain-enriched Ato-I RNA editing. Nature neuroscience 16, 1518-1522.

Li, J.Y., Lees-Murdock, D.J., Xu, G.L., and Walsh, C.P. (2004). Timing of establishment of paternal methylation imprints in the mouse. Genomics $84,952-960$.

Li, L.C., and Dahiya, R. (2002). MethPrimer: designing primers for methylation PCRs.

Bioinformatics 18, 1427-1431.

Li, X., Ito, M., Zhou, F., Youngson, N., Zuo, X., Leder, P., and Ferguson-Smith, A.C. (2008). A maternal-zygotic effect gene, Zfp57, maintains both maternal and paternal imprints. Dev Cell 15, 547-557.

Liao, J., Karnik, R., Gu, H., Ziller, M.J., Clement, K., Tsankov, A.M., Akopian, V., Gifford, C.A., Donaghey, J., Galonska, C., et al. (2015). Targeted disruption of DNMT1, DNMT3A and DNMT3B in human embryonic stem cells. Nat Genet 47, 469-478.

Liddicoat, B.J., Piskol, R., Chalk, A.M., Ramaswami, G., Higuchi, M., Hartner, J.C., Li, J.B., Seeburg, P.H., and Walkley, C.R. (2015). RNA editing by ADAR1 prevents MDA5 sensing of endogenous dsRNA as nonself. Science 349, 1115-1120. 
Lim, D., Bowdin, S.C., Tee, L., Kirby, G.A., Blair, E., Fryer, A., Lam, W., Oley, C., Cole, T., Brueton, L.A., et al. (2009). Clinical and molecular genetic features of Beckwith-Wiedemann syndrome associated with assisted reproductive technologies. Human reproduction 24, 741-747.

Lim, D.H., and Maher, E.R. (2010). Genomic imprinting syndromes and cancer. Adv Genet 70, $145-175$.

Lin, S.P., Youngson, N., Takada, S., Seitz, H., Reik, W., Paulsen, M., Cavaille, J., and FergusonSmith, A.C. (2003). Asymmetric regulation of imprinting on the maternal and paternal chromosomes at the Dlk1-Gtl2 imprinted cluster on mouse chromosome 12. Nat Genet 35, 97102.

Liu, Y., Siegmund, K.D., Laird, P.W., and Berman, B.P. (2012). Bis-SNP: Combined DNA methylation and SNP calling for Bisulfite-seq data. Genome biology 13, R61.

Lucifero, D., Mann, M.R., Bartolomei, M.S., and Trasler, J.M. (2004). Gene-specific timing and epigenetic memory in oocyte imprinting. Hum Mol Genet 13, 839-849.

Lucifero, D., Mertineit, C., Clarke, H.J., Bestor, T.H., and Trasler, J.M. (2002). Methylation dynamics of imprinted genes in mouse germ cells. Genomics 79, 530-538.

Luckett, W.P. (1978). Origin and differentiation of the yolk sac and extraembryonic mesoderm in presomite human and rhesus monkey embryos. Am J Anat 152, 59-97.

Ludwig, T., Eggenschwiler, J., Fisher, P., D'Ercole, A.J., Davenport, M.L., and Efstratiadis, A. (1996). Mouse mutants lacking the type 2 IGF receptor (IGF2R) are rescued from perinatal lethality in Igf2 and Igf1r null backgrounds. Developmental biology 177, 517-535.

Lyon, M.F. (1961). Gene action in the X-chromosome of the mouse (Mus musculus L.). Nature 190, 372-373.

Maatouk, D.M., Kellam, L.D., Mann, M.R., Lei, H., Li, E., Bartolomei, M.S., and Resnick, J.L. (2006). DNA methylation is a primary mechanism for silencing postmigratory primordial germ cell genes in both germ cell and somatic cell lineages. Development 133, 3411-3418.

MacDonald, W.A., and Mann, M.R. (2014). Epigenetic regulation of genomic imprinting from germ line to preimplantation. Mol Reprod Dev 81, 126-140.

MacHugh, D.E., Shriver, M.D., Loftus, R.T., Cunningham, P., and Bradley, D.G. (1997). Microsatellite DNA variation and the evolution, domestication and phylogeography of taurine and zebu cattle (Bos taurus and Bos indicus). Genetics 146, 1071-1086.

Maeda, T., Higashimoto, K., Jozaki, K., Yatsuki, H., Nakabayashi, K., Makita, Y., Tonoki, H., Okamoto, N., Takada, F., Ohashi, H., et al. (2014). Comprehensive and quantitative multilocus methylation analysis reveals the susceptibility of specific imprinted differentially methylated 
regions to aberrant methylation in Beckwith-Wiedemann syndrome with epimutations. Genetics in medicine : official journal of the American College of Medical Genetics.

Maher, E.R., Brueton, L.A., Bowdin, S.C., Luharia, A., Cooper, W., Cole, T.R., Macdonald, F., Sampson, J.R., Barratt, C.L., Reik, W., et al. (2003). Beckwith-Wiedemann syndrome and assisted reproduction technology (ART). Journal of medical genetics 40, 62-64.

Mancini-DiNardo, D., Steele, S.J., Ingram, R.S., and Tilghman, S.M. (2003). A differentially methylated region within the gene Kcnq1 functions as an imprinted promoter and silencer. Hum Mol Genet 12, 283-294.

Mann, M.R., Lee, S.S., Doherty, A.S., Verona, R.I., Nolen, L.D., Schultz, R.M., and Bartolomei, M.S. (2004). Selective loss of imprinting in the placenta following preimplantation development in culture. Development 131, 3727-3735.

Market-Velker, B.A., Zhang, L., Magri, L.S., Bonvissuto, A.C., and Mann, M.R. (2010). Dual effects of superovulation: loss of maternal and paternal imprinted methylation in a dosedependent manner. Human molecular genetics 19, 36-51.

Martin, M. (2011). Cutadapt removes adapter sequences from high-throughput sequencing reads. EMBnetjournal 17, 10-12.

Matsuura, T., Sutcliffe, J.S., Fang, P., Galjaard, R.J., Jiang, Y.H., Benton, C.S., Rommens, J.M., and Beaudet, A.L. (1997). De novo truncating mutations in E6-AP ubiquitin-protein ligase gene (UBE3A) in Angelman syndrome. Nat Genet 15, 74-77.

Maxfield, E.K., Sinclair, K.D., Broadbent, P.J., McEvoy, T.G., Robinson, J.J., and Maltin, C.A. (1998). Short-term culture of ovine embryos modifies fetal myogenesis. Am J Physiol 274, E1121-1123.

McCarthy, D.J., Chen, Y., and Smyth, G.K. (2012). Differential expression analysis of multifactor RNA-Seq experiments with respect to biological variation. Nucleic acids research 40, 4288-4297.

McGrath, J., and Solter, D. (1984). Completion of mouse embryogenesis requires both the maternal and paternal genomes. Cell 37, 179-183.

McGraw, S., Oakes, C.C., Martel, J., Cirio, M.C., de Zeeuw, P., Mak, W., Plass, C., Bartolomei, M.S., Chaillet, J.R., and Trasler, J.M. (2013). Loss of DNMT1o disrupts imprinted X chromosome inactivation and accentuates placental defects in females. PLoS genetics 9 , e1003873.

McKenna, A., Hanna, M., Banks, E., Sivachenko, A., Cibulskis, K., Kernytsky, A., Garimella, K., Altshuler, D., Gabriel, S., Daly, M., et al. (2010). The Genome Analysis Toolkit: a MapReduce framework for analyzing next-generation DNA sequencing data. Genome research 20, 1297 1303. 
Meng, L., Person, R.E., and Beaudet, A.L. (2012). Ube3a-ATS is an atypical RNA polymerase II transcript that represses the paternal expression of Ube3a. Hum Mol Genet 21, 3001-3012.

Meng, L., Ward, A.J., Chun, S., Bennett, C.F., Beaudet, A.L., and Rigo, F. (2015). Towards a therapy for Angelman syndrome by targeting a long non-coding RNA. Nature 518, 409-412.

Merzouk, S., Deuve, J.L., Dubois, A., Navarro, P., Avner, P., and Morey, C. (2014). Lineagespecific regulation of imprinted $\mathrm{X}$ inactivation in extraembryonic endoderm stem cells.

Epigenetics Chromatin 7, 11.

Messerschmidt, D.M., de Vries, W., Ito, M., Solter, D., Ferguson-Smith, A., and Knowles, B.B. (2012). Trim 28 is required for epigenetic stability during mouse oocyte to embryo transition.

Science 335, 1499-1502.

Messerschmidt, D.M., Knowles, B.B., and Solter, D. (2014). DNA methylation dynamics during epigenetic reprogramming in the germline and preimplantation embryos. Genes \& development $28,812-828$.

Meyer, E., Lim, D., Pasha, S., Tee, L.J., Rahman, F., Yates, J.R., Woods, C.G., Reik, W., and Maher, E.R. (2009). Germline mutation in NLRP2 (NALP2) in a familial imprinting disorder (Beckwith-Wiedemann Syndrome). PLoS Genet 5, e1000423.

Mikkelsen, T.S., Ku, M., Jaffe, D.B., Issac, B., Lieberman, E., Giannoukos, G., Alvarez, P., Brockman, W., Kim, T.K., Koche, R.P., et al. (2007). Genome-wide maps of chromatin state in pluripotent and lineage-committed cells. Nature 448, 553-560.

Miri, K., Latham, K., Panning, B., Zhong, Z., Andersen, A., and Varmuza, S. (2013). The imprinted polycomb group gene Sfmbt2 is required for trophoblast maintenance and placenta development. Development 140, 4480-4489.

Miura, K., Kishino, T., Li, E., Webber, H., Dikkes, P., Holmes, G.L., and Wagstaff, J. (2002). Neurobehavioral and electroencephalographic abnormalities in Ube3a maternal-deficient mice. Neurobiol Dis 9, 149-159.

Miyoshi, N., Barton, S.C., Kaneda, M., Hajkova, P., and Surani, M.A. (2006). The continuing quest to comprehend genomic imprinting. Cytogenetic and genome research 113, 6-11.

Molyneaux, K.A., Stallock, J., Schaible, K., and Wylie, C. (2001). Time-lapse analysis of living mouse germ cell migration. Dev Biol 240, 488-498.

Monk, D., Arnaud, P., Apostolidou, S., Hills, F.A., Kelsey, G., Stanier, P., Feil, R., and Moore, G.E. (2006). Limited evolutionary conservation of imprinting in the human placenta. Proc Natl Acad Sci U S A 103, 6623-6628.

Monk, M., and McLaren, A. (1981). X-chromosome activity in foetal germ cells of the mouse. J Embryol Exp Morphol 63, 75-84. 
Monnier, P., Martinet, C., Pontis, J., Stancheva, I., Ait-Si-Ali, S., and Dandolo, L. (2013). H19 lncRNA controls gene expression of the Imprinted Gene Network by recruiting MBD1. Proc Natl Acad Sci U S A 110, 20693-20698.

Morgan, H.D., Santos, F., Green, K., Dean, W., and Reik, W. (2005). Epigenetic reprogramming in mammals. Human molecular genetics 14 Spec No 1, R47-58.

Morison, I.M., Ramsay, J.P., and Spencer, H.G. (2005). A census of mammalian imprinting. Trends in genetics : TIG 21, 457-465.

Munger, S.C., Raghupathy, N., Choi, K., Simons, A.K., Gatti, D.M., Hinerfeld, D.A., Svenson, K.L., Keller, M.P., Attie, A.D., Hibbs, M.A., et al. (2014). RNA-Seq alignment to individualized genomes improves transcript abundance estimates in multiparent populations. Genetics 198, 5973.

Muscatelli, F., Abrous, D.N., Massacrier, A., Boccaccio, I., Le Moal, M., Cau, P., and Cremer, H. (2000). Disruption of the mouse Necdin gene results in hypothalamic and behavioral alterations reminiscent of the human Prader-Willi syndrome. Hum Mol Genet 9, 3101-3110.

Mussa, A., Molinatto, C., Baldassarre, G., Riberi, E., Russo, S., Larizza, L., Riccio, A., and Ferrero, G.B. (2016). Cancer Risk in Beckwith-Wiedemann Syndrome: A Systematic Review and Meta-Analysis Outlining a Novel (Epi)Genotype Specific Histotype Targeted Screening Protocol. J Pediatr 176, 142-149 e141.

Nakamura, T., Arai, Y., Umehara, H., Masuhara, M., Kimura, T., Taniguchi, H., Sekimoto, T., Ikawa, M., Yoneda, Y., Okabe, M., et al. (2007). PGC7/Stella protects against DNA demethylation in early embryogenesis. Nat Cell Biol 9, 64-71.

Nakamura, T., Liu, Y.J., Nakashima, H., Umehara, H., Inoue, K., Matoba, S., Tachibana, M., Ogura, A., Shinkai, Y., and Nakano, T. (2012). PGC7 binds histone H3K9me2 to protect against conversion of $5 \mathrm{mC}$ to $5 \mathrm{hmC}$ in early embryos. Nature $486,415-419$.

Neeman, Y., Levanon, E.Y., Jantsch, M.F., and Eisenberg, E. (2006). RNA editing level in the mouse is determined by the genomic repeat repertoire. Rna 12, 1802-1809.

Niemann, H., Wrenzycki, C., Lucas-Hahn, A., Brambrink, T., Kues, W.A., and Carnwath, J.W. (2002). Gene expression patterns in bovine in vitro-produced and nuclear transfer-derived embryos and their implications for early development. Cloning and stem cells 4, 29-38.

Nishikura, K. (2010). Functions and regulation of RNA editing by ADAR deaminases. Annual review of biochemistry 79, 321-349.

O'Doherty, A.M., O'Shea, L.C., and Fair, T. (2012). Bovine DNA methylation imprints are established in an oocyte size-specific manner, which are coordinated with the expression of the DNMT3 family proteins. Biology of reproduction 86,67 . 
Obata, Y., and Kono, T. (2002). Maternal primary imprinting is established at a specific time for each gene throughout oocyte growth. J Biol Chem 277, 5285-5289.

Ohta, T., Gray, T.A., Rogan, P.K., Buiting, K., Gabriel, J.M., Saitoh, S., Muralidhar, B., Bilienska, B., Krajewska-Walasek, M., Driscoll, D.J., et al. (1999). Imprinting-mutation mechanisms in Prader-Willi syndrome. Am J Hum Genet 64, 397-413.

Okamura, K., Sakaki, Y., and Ito, T. (2005). Comparative genomics approach toward critical determinants for the imprinting of an evolutionarily conserved gene Impact. Biochemical and biophysical research communications 329, 824-830.

Ooi, S.K., Qiu, C., Bernstein, E., Li, K., Jia, D., Yang, Z., Erdjument-Bromage, H., Tempst, P., Lin, S.P., Allis, C.D., et al. (2007). DNMT3L connects unmethylated lysine 4 of histone H3 to de novo methylation of DNA. Nature 448, 714-717.

Pandey, R.R., Mondal, T., Mohammad, F., Enroth, S., Redrup, L., Komorowski, J., Nagano, T., Mancini-Dinardo, D., and Kanduri, C. (2008). Kenq1 ot1 antisense noncoding RNA mediates lineage-specific transcriptional silencing through chromatin-level regulation. Mol Cell 32, 232246.

Peters, J. (2014). The role of genomic imprinting in biology and disease: an expanding view. Nature reviews Genetics 15, 517-530.

Pettenati, M.J., Haines, J.L., Higgins, R.R., Wappner, R.S., Palmer, C.G., and Weaver, D.D. (1986). Wiedemann-Beckwith syndrome: presentation of clinical and cytogenetic data on 22 new cases and review of the literature. Hum Genet 74, 143-154.

Ping, A.J., Reeve, A.E., Law, D.J., Young, M.R., Boehnke, M., and Feinberg, A.P. (1989). Genetic linkage of Beckwith-Wiedemann syndrome to 11p15. Am J Hum Genet 44, 720-723.

Pinto, Y., Cohen, H.Y., and Levanon, E.Y. (2014). Mammalian conserved ADAR targets comprise only a small fragment of the human editosome. Genome biology 15, R5.

Piskol, R., Ramaswami, G., and Li, J.B. (2013). Reliable identification of genomic variants from RNA-seq data. American journal of human genetics 93, 641-651.

Plasschaert, R.N., and Bartolomei, M.S. (2014). Genomic imprinting in development, growth, behavior and stem cells. Development 141, 1805-1813.

Polanski, Z., Motosugi, N., Tsurumi, C., Hiiragi, T., and Hoffmann, S. (2008). Hypomethylation of paternal DNA in the late mouse zygote is not essential for development. Int J Dev Biol 52, 295-298.

Poole, R.L., Docherty, L.E., Al Sayegh, A., Caliebe, A., Turner, C., Baple, E., Wakeling, E., Harrison, L., Lehmann, A., Temple, I.K., et al. (2013). Targeted methylation testing of a patient 
cohort broadens the epigenetic and clinical description of imprinting disorders. American journal of medical genetics Part A 161, 2174-2182.

Pruitt, K.D., Brown, G.R., Hiatt, S.M., Thibaud-Nissen, F., Astashyn, A., Ermolaeva, O., Farrell, C.M., Hart, J., Landrum, M.J., McGarvey, K.M., et al. (2014). RefSeq: an update on mammalian reference sequences. Nucleic acids research 42, D756-763.

Quenneville, S., Verde, G., Corsinotti, A., Kapopoulou, A., Jakobsson, J., Offner, S., Baglivo, I., Pedone, P.V., Grimaldi, G., Riccio, A., et al. (2011). In embryonic stem cells, ZFP57/KAP1 recognize a methylated hexanucleotide to affect chromatin and DNA methylation of imprinting control regions. Mol Cell 44, 361-372.

Quinlan, A.R., and Hall, I.M. (2010). BEDTools: a flexible suite of utilities for comparing genomic features. Bioinformatics 26, 841-842.

Ramaswami, G., and Li, J.B. (2014). RADAR: a rigorously annotated database of A-to-I RNA editing. Nucleic acids research 42, D109-113.

Ramaswami, G., Lin, W., Piskol, R., Tan, M.H., Davis, C., and Li, J.B. (2012). Accurate identification of human Alu and non-Alu RNA editing sites. Nature methods 9, 579-581.

Ramaswami, G., Zhang, R., Piskol, R., Keegan, L.P., Deng, P., O'Connell, M.A., and Li, J.B. (2013). Identifying RNA editing sites using RNA sequencing data alone. Nature methods 10 , 128-132.

Ramirez, F., Dundar, F., Diehl, S., Gruning, B.A., and Manke, T. (2014). deepTools: a flexible platform for exploring deep-sequencing data. Nucleic acids research 42, W187-191.

Rinaudo, P., and Schultz, R.M. (2004). Effects of embryo culture on global pattern of gene expression in preimplantation mouse embryos. Reproduction 128, 301-311.

Ripoche, M.A., Kress, C., Poirier, F., and Dandolo, L. (1997). Deletion of the H19 transcription unit reveals the existence of a putative imprinting control element. Genes Dev 11, 1596-1604.

Rivera, R.M., Stein, P., Weaver, J.R., Mager, J., Schultz, R.M., and Bartolomei, M.S. (2008). Manipulations of mouse embryos prior to implantation result in aberrant expression of imprinted genes on day 9.5 of development. Human molecular genetics 17, 1-14.

Rizos, D., Ward, F., Duffy, P., Boland, M.P., and Lonergan, P. (2002). Consequences of bovine oocyte maturation, fertilization or early embryo development in vitro versus in vivo: implications for blastocyst yield and blastocyst quality. Molecular reproduction and development 61, 234-248.

Robbins, K.M., Chen, Z., Wells, K.D., and Rivera, R.M. (2012). Expression of KCNQ1OT1, CDKN1C, H19, and PLAGL1 and the methylation patterns at the KvDMR1 and H19/IGF2 imprinting control regions is conserved between human and bovine. Journal of biomedical science 19,95 . 
Robinson, J.T., Thorvaldsdottir, H., Winckler, W., Guttman, M., Lander, E.S., Getz, G., and Mesirov, J.P. (2011). Integrative genomics viewer. Nature biotechnology 29, 24-26.

Robinson, M.D., McCarthy, D.J., and Smyth, G.K. (2010). edgeR: a Bioconductor package for differential expression analysis of digital gene expression data. Bioinformatics 26, 139-140.

Robinson, M.D., and Oshlack, A. (2010). A scaling normalization method for differential expression analysis of RNA-seq data. Genome biology 11, R25.

Rockman, M.V., and Kruglyak, L. (2006). Genetics of global gene expression. Nature reviews Genetics 7, 862-872.

Rooke, J.A., McEvoy, T.G., Ashworth, C.J., Robinson, J.J., Wilmut, I., Young, L.E., and Sinclair, K.D. (2007). Ovine fetal development is more sensitive to perturbation by the presence of serum in embryo culture before rather than after compaction. Theriogenology 67, 639-647.

Rosenbloom, K.R., Armstrong, J., Barber, G.P., Casper, J., Clawson, H., Diekhans, M., Dreszer, T.R., Fujita, P.A., Guruvadoo, L., Haeussler, M., et al. (2015). The UCSC Genome Browser database: 2015 update. Nucleic Acids Res 43, D670-681.

Rossant, J., and Cross, J.C. (2001). Placental development: lessons from mouse mutants. Nat Rev Genet 2, 538-548.

Rougeulle, C., Cardoso, C., Fontes, M., Colleaux, L., and Lalande, M. (1998). An imprinted antisense RNA overlaps UBE3A and a second maternally expressed transcript. Nature genetics $19,15-16$.

Rueter, S.M., Dawson, T.R., and Emeson, R.B. (1999). Regulation of alternative splicing by RNA editing. Nature 399, 75-80.

Saitou, M., Barton, S.C., and Surani, M.A. (2002). A molecular programme for the specification of germ cell fate in mice. Nature 418, 293-300.

Sanli, I., and Feil, R. (2015). Chromatin mechanisms in the developmental control of imprinted gene expression. Int J Biochem Cell Biol 67, 139-147.

Sato, M., Kimura, T., Kurokawa, K., Fujita, Y., Abe, K., Masuhara, M., Yasunaga, T., Ryo, A., Yamamoto, M., and Nakano, T. (2002). Identification of PGC7, a new gene expressed specifically in preimplantation embryos and germ cells. Mech Dev 113, 91-94.

Schiaffino, S., and Mammucari, C. (2011). Regulation of skeletal muscle growth by the IGF1Akt/PKB pathway: insights from genetic models. Skelet Muscle 1, 4. 
Schmidt, M., Greve, T., Avery, B., Beckers, J.F., Sulon, J., and Hansen, H.B. (1996).

Pregnancies, calves and calf viability after transfer of in vitro produced bovine embryos.

Theriogenology 46, 527-539.

Schoenherr, C.J., Levorse, J.M., and Tilghman, S.M. (2003). CTCF maintains differential methylation at the Igf2/H19 locus. Nat Genet 33, 66-69.

Seisenberger, S., Andrews, S., Krueger, F., Arand, J., Walter, J., Santos, F., Popp, C., Thienpont, B., Dean, W., and Reik, W. (2012). The dynamics of genome-wide DNA methylation reprogramming in mouse primordial germ cells. Mol Cell 48, 849-862.

Seki, Y., Hayashi, K., Itoh, K., Mizugaki, M., Saitou, M., and Matsui, Y. (2005). Extensive and orderly reprogramming of genome-wide chromatin modifications associated with specification and early development of germ cells in mice. Dev Biol 278, 440-458.

Sekita, Y., Wagatsuma, H., Nakamura, K., Ono, R., Kagami, M., Wakisaka, N., Hino, T., SuzukiMigishima, R., Kohda, T., Ogura, A., et al. (2008). Role of retrotransposon-derived imprinted gene, Rtl1, in the feto-maternal interface of mouse placenta. Nat Genet 40, 243-248.

Shen, L., Inoue, A., He, J., Liu, Y., Lu, F., and Zhang, Y. (2014). Tet3 and DNA replication mediate demethylation of both the maternal and paternal genomes in mouse zygotes. Cell Stem Cell 15, 459-470.

Shen, L., Wu, H., Diep, D., Yamaguchi, S., D'Alessio, A.C., Fung, H.L., Zhang, K., and Zhang, Y. (2013). Genome-wide analysis reveals TET- and TDG-dependent 5-methylcytosine oxidation dynamics. Cell 153, 692-706.

Shi, J., Luo, L., Eash, J., Ibebunjo, C., and Glass, D.J. (2011). The SCF-Fbxo40 complex induces IRS1 ubiquitination in skeletal muscle, limiting IGF1 signaling. Developmental cell 21, 835-847.

Shirane, K., Toh, H., Kobayashi, H., Miura, F., Chiba, H., Ito, T., Kono, T., and Sasaki, H. (2013). Mouse oocyte methylomes at base resolution reveal genome-wide accumulation of nonCpG methylation and role of DNA methyltransferases. PLoS Genet 9, e1003439.

Silva-Santos, S., van Woerden, G.M., Bruinsma, C.F., Mientjes, E., Jolfaei, M.A., Distel, B., Kushner, S.A., and Elgersma, Y. (2015). Ube3a reinstatement identifies distinct developmental windows in a murine Angelman syndrome model. J Clin Invest 125, 2069-2076.

Sinclair, K.D., McEvoy, T.G., Maxfield, E.K., Maltin, C.A., Young, L.E., Wilmut, I., Broadbent, P.J., and Robinson, J.J. (1999). Aberrant fetal growth and development after in vitro culture of sheep zygotes. J Reprod Fertil 116, 177-186.

Sinclair, K.D., Young, L.E., Wilmut, I., and McEvoy, T.G. (2000). In-utero overgrowth in ruminants following embryo culture: lessons from mice and a warning to men. Human Reproduction 15, 68-86. 
Singh, P., Li, A.X., Tran, D.A., Oates, N., Kang, E.R., Wu, X., and Szabo, P.E. (2013). De novo DNA methylation in the male germ line occurs by default but is excluded at sites of H3K4 methylation. Cell Rep 4, 205-219.

Sleutels, F., Zwart, R., and Barlow, D.P. (2002). The non-coding Air RNA is required for silencing autosomal imprinted genes. Nature 415, 810-813.

Smallwood, S.A., Tomizawa, S., Krueger, F., Ruf, N., Carli, N., Segonds-Pichon, A., Sato, S., Hata, K., Andrews, S.R., and Kelsey, G. (2011). Dynamic CpG island methylation landscape in oocytes and preimplantation embryos. Nat Genet 43, 811-814.

Smilinich, N.J., Day, C.D., Fitzpatrick, G.V., Caldwell, G.M., Lossie, A.C., Cooper, P.R., Smallwood, A.C., Joyce, J.A., Schofield, P.N., Reik, W., et al. (1999). A maternally methylated CpG island in KvLQT1 is associated with an antisense paternal transcript and loss of imprinting in Beckwith-Wiedemann syndrome. Proc Natl Acad Sci U S A 96, 8064-8069.

Smith, E.Y., Futtner, C.R., Chamberlain, S.J., Johnstone, K.A., and Resnick, J.L. (2011). Transcription is required to establish maternal imprinting at the Prader-Willi syndrome and Angelman syndrome locus. PLoS Genet 7, e1002422.

Smith, Z.D., Chan, M.M., Mikkelsen, T.S., Gu, H., Gnirke, A., Regev, A., and Meissner, A. (2012). A unique regulatory phase of DNA methylation in the early mammalian embryo. Nature $484,339-344$.

Smith, Z.D., and Meissner, A. (2013). DNA methylation: roles in mammalian development. Nat Rev Genet 14, 204-220.

Sparago, A., Cerrato, F., Vernucci, M., Ferrero, G.B., Silengo, M.C., and Riccio, A. (2004). Microdeletions in the human H19 DMR result in loss of IGF2 imprinting and BeckwithWiedemann syndrome. Nat Genet 36, 958-960.

Stevenson, K.R., Coolon, J.D., and Wittkopp, P.J. (2013). Sources of bias in measures of allelespecific expression derived from RNA-sequence data aligned to a single reference genome. BMC genomics 14, 536 .

Stewart, K.R., Veselovska, L., and Kelsey, G. (2016). Establishment and functions of DNA methylation in the germline. Epigenomics 8, 1399-1413.

Stewart, K.R., Veselovska, L., Kim, J., Huang, J., Saadeh, H., Tomizawa, S., Smallwood, S.A., Chen, T., and Kelsey, G. (2015). Dynamic changes in histone modifications precede de novo DNA methylation in oocytes. Genes Dev 29, 2449-2462.

Stoger, R., Kubicka, P., Liu, C.G., Kafri, T., Razin, A., Cedar, H., and Barlow, D.P. (1993). Maternal-specific methylation of the imprinted mouse Igf2r locus identifies the expressed locus as carrying the imprinting signal. Cell 73,61-71. 
Storey, J. (2002). A direct approach to false discovery rates. Journal of the Royal Statistical Society 64, 479-498.

Sun, F.L., Dean, W.L., Kelsey, G., Allen, N.D., and Reik, W. (1997). Transactivation of Igf2 in a mouse model of Beckwith-Wiedemann syndrome. Nature 389, 809-815.

Susiarjo, M., Sasson, I., Mesaros, C., and Bartolomei, M.S. (2013). Bisphenol a exposure disrupts genomic imprinting in the mouse. PLoS genetics 9, e1003401.

Szabo, P.E., Tang, S.H., Silva, F.J., Tsark, W.M., and Mann, J.R. (2004). Role of CTCF binding sites in the Igf2/H19 imprinting control region. Mol Cell Biol 24, 4791-4800.

Tahiliani, M., Koh, K.P., Shen, Y., Pastor, W.A., Bandukwala, H., Brudno, Y., Agarwal, S., Iyer, L.M., Liu, D.R., Aravind, L., et al. (2009). Conversion of 5-methylcytosine to 5-

hydroxymethylcytosine in mammalian DNA by MLL partner TET1. Science 324, 930-935.

Talbot, N.C., Caperna, T.J., Edwards, J.L., Garrett, W., Wells, K.D., and Ealy, A.D. (2000). Bovine blastocyst-derived trophectoderm and endoderm cell cultures: interferon tau and transferrin expression as respective in vitro markers. Biology of reproduction 62, 235-247.

Tam, P.P., and Snow, M.H. (1981). Proliferation and migration of primordial germ cells during compensatory growth in mouse embryos. J Embryol Exp Morphol 64, 133-147.

Tam, P.P., Zhou, S.X., and Tan, S.S. (1994). X-chromosome activity of the mouse primordial germ cells revealed by the expression of an X-linked lacZ transgene. Development 120, 29252932.

Tee, L., Lim, D.H., Dias, R.P., Baudement, M.O., Slater, A.A., Kirby, G., Hancocks, T., Stewart, H., Hardy, C., Macdonald, F., et al. (2013). Epimutation profiling in Beckwith-Wiedemann syndrome: relationship with assisted reproductive technology. Clinical epigenetics 5, 23.

Terranova, R., Yokobayashi, S., Stadler, M.B., Otte, A.P., van Lohuizen, M., Orkin, S.H., and Peters, A.H. (2008). Polycomb group proteins Ezh2 and Rnf2 direct genomic contraction and imprinted repression in early mouse embryos. Dev Cell 15, 668-679.

Thompson, J.G. (1997). Comparison between in vivo-derived and in vitro-produced preelongation embryos from domestic ruminants. Reproduction, fertility, and development 9, 341354.

Thorsteinsdottir, S., Deries, M., Cachaco, A.S., and Bajanca, F. (2011). The extracellular matrix dimension of skeletal muscle development. Developmental biology 354, 191-207.

Thorvaldsen, J.L., Duran, K.L., and Bartolomei, M.S. (1998). Deletion of the H19 differentially methylated domain results in loss of imprinted expression of H19 and Igf2. Genes Dev 12, 36933702 . 
Tian, X.C. (2014). Genomic imprinting in farm animals. Annu Rev Anim Biosci 2, 23-40.

Trapnell, C., Williams, B.A., Pertea, G., Mortazavi, A., Kwan, G., van Baren, M.J., Salzberg, S.L., Wold, B.J., and Pachter, L. (2010). Transcript assembly and quantification by RNA-Seq reveals unannotated transcripts and isoform switching during cell differentiation. Nat Biotechnol 28, 511-515.

Tremblay, K.D., Saam, J.R., Ingram, R.S., Tilghman, S.M., and Bartolomei, M.S. (1995). A paternal-specific methylation imprint marks the alleles of the mouse H19 gene. Nat Genet 9, 407413.

Tunster, S.J., Van de Pette, M., and John, R.M. (2011). Fetal overgrowth in the Cdkn1c mouse model of Beckwith-Wiedemann syndrome. Disease models \& mechanisms 4, 814-821.

Tycko, B. (2010). Allele-specific DNA methylation: beyond imprinting. Human molecular genetics 19, R210-220.

Uechi, H., Tsutsumi, O., Morita, Y., and Taketani, Y. (1997). Cryopreservation of mouse embryos affects later embryonic development possibly through reduced expression of the glucose transporter GLUT1. Mol Reprod Dev 48, 496-500.

Umlauf, D., Goto, Y., Cao, R., Cerqueira, F., Wagschal, A., Zhang, Y., and Feil, R. (2004). Imprinting along the Kcnq1 domain on mouse chromosome 7 involves repressive histone methylation and recruitment of Polycomb group complexes. Nat Genet 36, 1296-1300.

Valente, K.D., Varela, M.C., Koiffmann, C.P., Andrade, J.Q., Grossmann, R., Kok, F., and Marques-Dias, M.J. (2013). Angelman syndrome caused by deletion: a genotype-phenotype correlation determined by breakpoint. Epilepsy Res 105, 234-239.

van Leeuwen, J., Berg, D.K., and Pfeffer, P.L. (2015). Morphological and Gene Expression Changes in Cattle Embryos from Hatched Blastocyst to Early Gastrulation Stages after Transfer of In Vitro Produced Embryos. PloS one 10, e0129787.

Varrault, A., Gueydan, C., Delalbre, A., Bellmann, A., Houssami, S., Aknin, C., Severac, D., Chotard, L., Kahli, M., Le Digarcher, A., et al. (2006). Zac1 regulates an imprinted gene network critically involved in the control of embryonic growth. Dev Cell 11, 711-722.

Vermeiden, J.P., and Bernardus, R.E. (2013). Are imprinting disorders more prevalent after human in vitro fertilization or intracytoplasmic sperm injection? Fertility and sterility 99, 642651 .

Veselovska, L., Smallwood, S.A., Saadeh, H., Stewart, K.R., Krueger, F., Maupetit-Mehouas, S., Arnaud, P., Tomizawa, S., Andrews, S., and Kelsey, G. (2015). Deep sequencing and de novo assembly of the mouse oocyte transcriptome define the contribution of transcription to the DNA methylation landscape. Genome Biol 16, 209. 
Wake, N., Takagi, N., and Sasaki, M. (1976). Non-random inactivation of X chromosome in the rat yolk sac. Nature 262, 580-581.

Walker, S.K., Hartwich, K.M., and Seamark, R.F. (1996). The production of unusually large offspring following embryo manipulation: Concepts and challenges. Theriogenology 45, 111-120.

Wan, L.B., and Bartolomei, M.S. (2008). Regulation of imprinting in clusters: noncoding RNAs versus insulators. Adv Genet 61, 207-223.

Wang, L., Park, H.J., Dasari, S., Wang, S., Kocher, J.P., and Li, W. (2013). CPAT: CodingPotential Assessment Tool using an alignment-free logistic regression model. Nucleic acids research $41, \mathrm{e} 74$.

Wang, X., and Clark, A.G. (2014). Using next-generation RNA sequencing to identify imprinted genes. Heredity 113, 156-166.

Wang, X., Miller, D.C., Clark, A.G., and Antczak, D.F. (2012). Random X inactivation in the mule and horse placenta. Genome research 22, 1855-1863.

Wang, X., Sun, Q., McGrath, S.D., Mardis, E.R., Soloway, P.D., and Clark, A.G. (2008). Transcriptome-wide identification of novel imprinted genes in neonatal mouse brain. PloS one 3, e3839.

Watanabe, T., Tomizawa, S., Mitsuya, K., Totoki, Y., Yamamoto, Y., Kuramochi-Miyagawa, S., Iida, N., Hoki, Y., Murphy, P.J., Toyoda, A., et al. (2011). Role for piRNAs and noncoding RNA in de novo DNA methylation of the imprinted mouse Rasgrf1 locus. Science 332, 848-852.

Webster, K.E., O'Bryan, M.K., Fletcher, S., Crewther, P.E., Aapola, U., Craig, J., Harrison, D.K., Aung, H., Phutikanit, N., Lyle, R., et al. (2005). Meiotic and epigenetic defects in Dnmt3Lknockout mouse spermatogenesis. Proc Natl Acad Sci U S A 102, 4068-4073.

Weick, E.M., and Miska, E.A. (2014). piRNAs: from biogenesis to function. Development 141, 3458-3471.

Weksberg, R., Shuman, C., and Beckwith, J.B. (2010). Beckwith-Wiedemann syndrome. European journal of human genetics : EJHG 18, 8-14.

Weksberg, R., Shuman, C., and Smith, A.C. (2005). Beckwith-Wiedemann syndrome. American journal of medical genetics Part C, Seminars in medical genetics 137C, 12-23.

Whitacre, L.K., Tizioto, P.C., Kim, J., Sonstegard, T.S., Schroeder, S.G., Alexander, L.J., Medrano, J.F., Schnabel, R.D., Taylor, J.F., and Decker, J.E. (2015). What's in your nextgeneration sequence data? An exploration of unmapped DNA and RNA sequence reads from the bovine reference individual. BMC genomics 16, 1114. 
Wiedmann, H. (1964). Complexe malformatif familial avechernie ombilicale et markroglossie: un syndrome nouveau. J Genet Hum 13, 223-232.

Williamson, C.M., Turner, M.D., Ball, S.T., Nottingham, W.T., Glenister, P., Fray, M., Tymowska-Lalanne, Z., Plagge, A., Powles-Glover, N., Kelsey, G., et al. (2006). Identification of an imprinting control region affecting the expression of all transcripts in the Gnas cluster. Nat Genet 38, 350-355.

Wrenzycki, C., Herrmann, D., Carnwath, J.W., and Niemann, H. (1998). Expression of RNA from developmentally important genes in preimplantation bovine embryos produced in TCM supplemented with BSA. Journal of reproduction and fertility 112, 387-398.

Wrenzycki, C., Herrmann, D., Keskintepe, L., Martins, A., Jr., Sirisathien, S., Brackett, B., and Niemann, H. (2001). Effects of culture system and protein supplementation on mRNA expression in pre-implantation bovine embryos. Human reproduction (Oxford, England) 16, 893-901.

Wrenzycki, C., Herrmann, D., Lucas-Hahn, A., Lemme, E., Korsawe, K., and Niemann, H. (2004). Gene expression patterns in in vitro-produced and somatic nuclear transfer-derived preimplantation bovine embryos: relationship to the large offspring syndrome? Animal reproduction science $82-83,593-603$.

Wrenzycki, C., Lucas-Hahn, A., Herrmann, D., Lemme, E., Korsawe, K., and Niemann, H. (2002). In vitro production and nuclear transfer affect dosage compensation of the X-linked gene transcripts G6PD, PGK, and Xist in preimplantation bovine embryos. Biology of reproduction 66, 127-134.

Wutz, A., Smrzka, O.W., Schweifer, N., Schellander, K., Wagner, E.F., and Barlow, D.P. (1997). Imprinted expression of the Igf2r gene depends on an intronic $\mathrm{CpG}$ island. Nature 389, 745-749.

Xie, W., Barr, C.L., Kim, A., Yue, F., Lee, A.Y., Eubanks, J., Dempster, E.L., and Ren, B. (2012). Base-resolution analyses of sequence and parent-of-origin dependent DNA methylation in the mouse genome. Cell 148, 816-831.

Xu, Y., Goodyer, C.G., Deal, C., and Polychronakos, C. (1993). Functional polymorphism in the parental imprinting of the human IGF2R gene. Biochemical and biophysical research communications $197,747-754$.

Xue, F., Tian, X.C., Du, F., Kubota, C., Taneja, M., Dinnyes, A., Dai, Y., Levine, H., Pereira, L.V., and Yang, X. (2002). Aberrant patterns of X chromosome inactivation in bovine clones. Nature genetics 31, 216-220.

Yamaguchi, S., Hong, K., Liu, R., Inoue, A., Shen, L., Zhang, K., and Zhang, Y. (2013a). Dynamics of 5-methylcytosine and 5-hydroxymethylcytosine during germ cell reprogramming. Cell Res 23, 329-339. 
Yamaguchi, S., Shen, L., Liu, Y., Sendler, D., and Zhang, Y. (2013b). Role of Tet1 in erasure of genomic imprinting. Nature 504, 460-464.

Yamasaki, K., Joh, K., Ohta, T., Masuzaki, H., Ishimaru, T., Mukai, T., Niikawa, N., Ogawa, M., Wagstaff, J., and Kishino, T. (2003). Neurons but not glial cells show reciprocal imprinting of sense and antisense transcripts of Ube3a. Human molecular genetics 12, 837-847.

Yan, Y., Frisen, J., Lee, M.H., Massague, J., and Barbacid, M. (1997). Ablation of the CDK inhibitor $\mathrm{p} 57 \mathrm{Kip} 2$ results in increased apoptosis and delayed differentiation during mouse development. Genes Dev 11, 973-983.

Yang, T., Adamson, T.E., Resnick, J.L., Leff, S., Wevrick, R., Francke, U., Jenkins, N.A., Copeland, N.G., and Brannan, C.I. (1998). A mouse model for Prader-Willi syndrome imprintingcentre mutations. Nat Genet 19, 25-31.

Young, L.E., Fernandes, K., McEvoy, T.G., Butterwith, S.C., Gutierrez, C.G., Carolan, C., Broadbent, P.J., Robinson, J.J., Wilmut, I., and Sinclair, K.D. (2001). Epigenetic change in IGF2R is associated with fetal overgrowth after sheep embryo culture. Nature genetics $27,153-$ 154.

Young, L.E., Schnieke, A.E., McCreath, K.J., Wieckowski, S., Konfortova, G., Fernandes, K., Ptak, G., Kind, A.J., Wilmut, I., Loi, P., et al. (2003). Conservation of IGF2-H19 and IGF2R imprinting in sheep: effects of somatic cell nuclear transfer. Mechanisms of development 120, $1433-1442$.

Young, L.E., Sinclair, K.D., and Wilmut, I. (1998). Large offspring syndrome in cattle and sheep. Reviews of reproduction 3, 155-163.

Yuan, S., Johnston, H.R., Zhang, G., Li, Y., Hu, Y.J., and Qin, Z.S. (2015). One Size Doesn't Fit All - RefEditor: Building Personalized Diploid Reference Genome to Improve Read Mapping and Genotype Calling in Next Generation Sequencing Studies. PLoS computational biology 11, e1004448.

Zaitoun, I., and Khatib, H. (2006). Assessment of genomic imprinting of SLC38A4, NNAT, NAP1L5, and H19 in cattle. BMC genetics 7, 49.

Zaitoun, I., and Khatib, H. (2008). Comparative genomic imprinting and expression analysis of six cattle genes. J Anim Sci 86, 25-32.

Zhang, P., Liegeois, N.J., Wong, C., Finegold, M., Hou, H., Thompson, J.C., Silverman, A., Harper, J.W., DePinho, R.A., and Elledge, S.J. (1997). Altered cell differentiation and proliferation in mice lacking p57KIP2 indicates a role in Beckwith-Wiedemann syndrome. Nature $387,151-158$. 
Zheng, H., Huang, B., Zhang, B., Xiang, Y., Du, Z., Xu, Q., Li, Y., Wang, Q., Ma, J., Peng, X., et al. (2016). Resetting Epigenetic Memory by Reprogramming of Histone Modifications in Mammals. Mol Cell 63, 1066-1079.

Zuo, X., Sheng, J., Lau, H.T., McDonald, C.M., Andrade, M., Cullen, D.E., Bell, F.T., Iacovino, M., Kyba, M., Xu, G., et al. (2012). Zinc finger protein ZFP57 requires its co-factor to recruit DNA methyltransferases and maintains DNA methylation imprint in embryonic stem cells via its transcriptional repression domain. J Biol Chem 287, 2107-2118. 


\section{VITA}

Zhiyuan Chen was born on April 23rd in 1990 in Shanxi Province in China. Chen grew up with his parents Cunliang Chen (Dad) and Xiaoying Fan (Mom). Chen attended China Agricultural University (CAU) in Beijing during 2007-2011. In June of 2011, Chen graduated from CAU with a bachelor degree in Animal Sciences. In August of 2011, Chen came to the University of Missouri-Columbia and joined Dr. Rivera's laboratory to pursue a Master of Science degree in the area of developmental epigenetics. After he completed the M.S. degree in May of 2013, Chen continued his Ph. D in the Rivera laboratory and worked on the molecular characterization of large offspring syndrome. After obtaining his Ph. D degree in May of 2017, Chen will become a postdoctoral research fellow at Yi Zhang's laboratory affiliated with Howard Hughes Medical Institute (HHMI), Harvard Medical School (HMS), and Boston Children's Hospital (BCH). 